\title{
ON THE SOLUTIONS OF HOLONOMIC THIRD-ORDER LINEAR IRREDUCIBLE DIFFERENTIAL EQUATIONS IN TERMS OF HYPERGEOMETRIC FUNCTIONS
}

\author{
By \\ MOUAFO WOUODJIE Merlin \\ zur Erlangung des akademischen Grades \\ eines Doktors der Naturwissenschaften \\ (Dr. rer. nat.) \\ im Fachbereich Mathematik und Naturwissenschaften \\ der Universität Kassel
}

Ph.D. thesis co-supervised by:

Prof. Dr. Wolfram Koepf

University of Kassel, Germany

and

Prof. Dr. Mama Foupouagnigni

University of Yaounde I, Cameroon. 


\section{Tag der Disputation:}

22. Februar 2018

\section{Erstgutachter:}

Prof. Dr. Wolfram Koepf

Universität Kassel

\section{Zweitgutachter:}

Prof. Dr. Mama Foupouagnigni University of Yaounde I, Cameroon

Prof. Dr. Mark van Hoeij

Florida State University

Tallahassee, USA 


\section{Dedication}

To my beloved Mother Kengné Victorine. May her soul rest in peace in the abode of Almighty God. 


\section{Acknowledgments}

This $\mathrm{PhD}$ thesis is a work that requires a lot of effort, time, patience and courage. That's why, at the end, I would like to take this opportunity to thank all those who helped me in one way or another to realize this work.

First of all, I would like to thank God, the almighty, for the grace, the courage and most especially the love he has put on me. I admit that nothing could have been possible without his inspiration, his protection and forgiveness.

I would like to express my sincere thanks to Professor Mama Foupouagnigni and Professor Wolfram Koepf who gave the topic of this work and put at my disposal their time, availability, all the books I needed, their constant encouragement, affectionate attitude, understanding, patience and also healthy criticism added considerably to my experience. You have kept on promoting my work and direct my thoughts. Thank you very much for the efforts and sacrifices made to co-supervise this work.

With great appreciation, a very special gratitude goes out to Professor Mark van Hoeij from the University of Florida who helped me with some problems that I got in my code implementations. Sincere thank for your advices, help and the motivation you gave me.

I acknowledge the financial support and the logistics from the Humboldt Laboratory for Computational and Educational Mathematics in Cameroon.

I sincerely thank AIMS (African Institute for Mathematics and Sciences) for the opportunity for studying and being tutor at AIMS-Cameroon.

I express my gratitude to DAAD scholarship (German Academic Exchange Service) which allowed me to come here to Germany to do my thesis. Thank you for helping and providing the funding for this work, for the pleasant working conditions and for all your organized conferences and advices.

I sincerely thank the University of Kassel for the "Promotions-Abschlussstipendium" which allowed me to finalize this work and to give also the best of me for that. Thank you for the financial support.

I am grateful to the Institute of Mathematics of the University of Kassel, for accepting me as a $\mathrm{PhD}$ student by giving me the opportunity to do research and providing invaluable guidance throughout this work.

I would like to thank administrative and technical staff members, professors and the research staff of the Institute of Mathematics of the University of Kassel for their kindness, advices and help.

The greatest of my acknowledgements goes to my parents whose patience and faith never failed throughout my academic process and their moral support. Special thanks to my mother Kengné Victorine who died after one month I arrived in Germany to start this thesis. Of all the gifts that life has offered me, you are the exceptional one, mother. Thank you very much for all your infinite prayers, encouragement and intersections for me from the sky where you rest in the peace and love of God. Amen. 
My brothers and sisters, you have helped me in other ways to realize this work, thank you for all the support you have given me throughout my studies.

All the members of my family, thank you for providing help and encouragement when needed.

Dr. Daniel Duviol Tcheutia, Dr. Etienne Le Grand Nana Chiadjeu and other friends, thank you for all the moments spent together and for your assistance without judgement.

And finally, last but by no means least, I sincerely thank also all those who contributed to my work/education and who could not explicitly be cited because of space limitation. 



\section{Contents}

Dedication $\quad$ i

Acknowledgments $\quad$ ii

Abstract $\quad$ ix

List of abbreviations $\quad \mathrm{x}$

0 General Introduction 1

1 Preliminaries $\quad 7$

1.1 Differential Operators and Singularities . . . . . . . . . . . . . 7

1.1.1 Differential Operators . . . . . . . . . . . . . . 7

1.1 .2 Singularities . . . . . . . . . . . . . . . . . . . . 9

1.1.3 Singular Points . . . . . . . . . . . . . . . . . . 9

1.2 Hypergeometric Series . . . . . . . . . . . . . . . . . . . 10

1.3 Bessel Functions . . . . . . . . . . . . . . . . . . . . 10

1.3.1 Bessel Operators . . . . . . . . . . . . . . . . . . . . 12

1.4 Formal Solutions . . . . . . . . . . . . . . . . 13

1.5 Maple Commands . . . . . . . . . . . . . . . . . . . . . . 18

1.5.1 From an operator to a differential equation and vice versa . . . . . 18

1.5.2 Solving linear differential equations . . . . . . . . . . . . . . . 19

1.5.3 Factorization into irreducible linear differential operators . . . . . . 19

1.5.4 Generalized Exponents . . . . . . . . . . . . . . . . 19

1.5.5 Formal and logarithmic solutions . . . . . . . . . . . . . 20

2 Transformations $\quad 21$

2.1 Types of Transformations . . . . . . . . . . . . . . . . 21

2.2 Examples . . . . . . . . . . . . . . . . . . 25

2.3 Transformations as relations . . . . . . . . . . . . . . . 26

2.4 Relation between Transformations and Generalized Exponents . . . . . . . 27

2.4.1 Relation between Exp-product Transformation and Generalized Exponents ........................... 28

2.4.2 Relation between Gauge Transformation and Generalized Exponents 34

2.4.3 Relation between Change of Variable Transformation and Generalized Exponents . . . . . . . . . . . . . . . . . . . . . 39 
3 Steps to Find Solutions

3.1 Meaning of our Problem . . . . . . . . . . . . . . . . . 54

3.2 The first step to solve our problem . . . . . . . . . . . . . 57

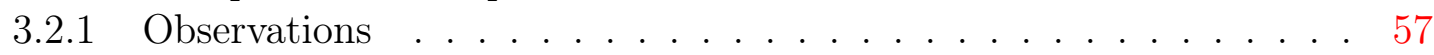

3.2 .2 Exponent differences . . . . . . . . . . . . . . . . . 67

3.3 The second step to solve our problem . . . . . . . . . . . . . . 73

3.3.1 The $p$-curvature test . . . . . . . . . . . . . . . . . 73

3.3.2 The Exp-product Equivalence . . . . . . . . . . . . . . . . . . . 75

3.3 .3 The Gauge Equivalence . . . . . . . . . . . . . . . . . . . . . 78

3.3.4 The Projective Equivalence . . . . . . . . . . . . . . . . . 80

4 Bessel Square Root Type Solutions $\quad 91$

4.1 Change of variable parameters are squares of rational functions in $k(x)$. . 91

4.1 .1 Exponent differences . . . . . . . . . . . . . . . . . 92

4.1.2 Parameter $f$ up to a constant . . . . . . . . . . . . . . . . . 94

4.1.3 No zero of $f$ is known $\left(\mathrm{S}_{\mathrm{reg}}(\mathrm{L})=\emptyset\right) \ldots \ldots \ldots \ldots$

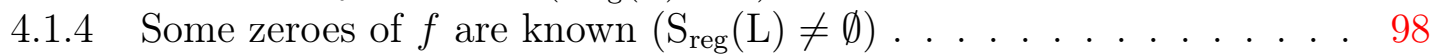

4.1.5 Example . . . . . . . . . . . . . . . . . 105

4.2 Change of variable parameters are not squares of rational functions in $k(x) 108$

4.2.1 Exponent differences . . . . . . . . . . . . . . . 108

4.2 .2 Truncated series for $f \ldots \ldots$. . . . . . . . . . . . 110

4.2.3 How to compute the denominator B of $f \ldots \ldots . . . . . . . .111$

4.2.4 How to get a bound for the degree of the numerator A of $f$. . . . 111

4.2.5 How to get linear equations for the coefficients of the numerator $\mathrm{A}$

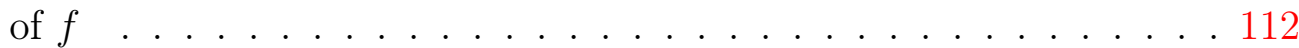

4.2.6 How to compute the numerator A of $f$ and the Bessel parameter $\nu 116$

4.2 .7 Example . . . . . . . . . . . . . . . . . . . . . . 131

4.2.8 Uniqueness of the Change of Variables Parameter $f$. . . . . . . . 134

4.3 Algebraic Extension . . . . . . . . . . . . . . . . . 135

$5{ }_{2} F_{2},{ }_{1} F_{2},{ }_{0} F_{2}$ and ${ }_{1} F_{1}^{2}$ Type Solutions 137

5.1 Some Help Tools . . . . . . . . . . . . . . . . . . . . . . . 137

5.1 .1 Properties . . . . . . . . . . . . . . . . . . 137

5.2 Exponent Differences . . . . . . . . . . . . . . . . . . . . 140

5.3 How to find the lower parameters $b_{1}$ and $b_{2}$ or $b$ of $F_{0}$ when $S_{\text {reg }}(\mathrm{L}) \neq \emptyset \ldots 144$

$5.3 .1 \quad \mathrm{~S}_{\mathrm{reg}}(\mathrm{L}) \neq \emptyset \ldots \ldots \ldots \ldots \ldots \ldots . \ldots \ldots . \ldots \ldots$

$5.3 .2 \mathrm{~S}_{\mathrm{reg}}(\mathrm{L})=\emptyset \ldots \ldots \ldots \ldots \ldots \ldots$

$5.4{ }_{2} F_{2}$ and ${ }_{1} F_{1}^{2}$ Type Solutions . . . . . . . . . . . . . . . . . 149

5.4.1 Parameter $f$ up to a constant . . . . . . . . . . . . . . . 149

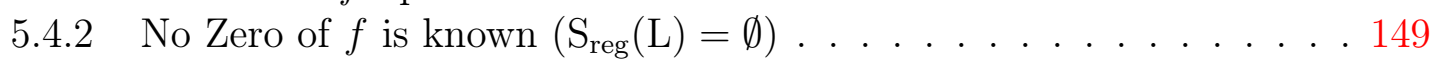

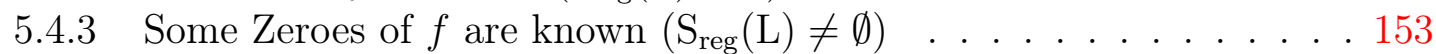

5.4.4 Examples .......................... 154

$5.5{ }_{1} F_{2}$ and ${ }_{0} F_{2}$ type solutions $\ldots \ldots \ldots \ldots \ldots$

5.5.1 Truncated series for $f \ldots \ldots \ldots 161$

5.5.2 How to compute the denominator B of $f \ldots . . . . . . .161$

5.5.3 How to get a bound for the degree of the numerator A of $f$. . . . 161

5.5.4 How to get linear equations for the coefficients of the numerator A

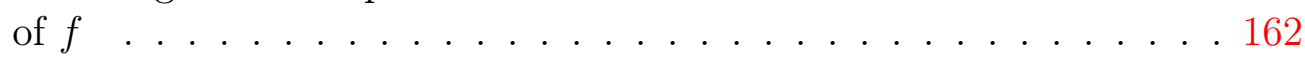


5.5.5 How to compute the upper parameter $a_{1}$ when $\mathrm{L}_{0}=\mathrm{L}_{12} \quad \ldots . . .163$

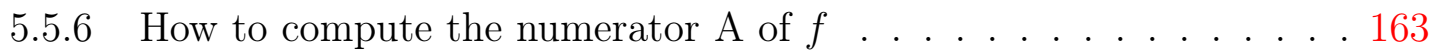

5.5.7 Examples ........................... 165

6 Conclusion and Perspectives 173

$\begin{array}{ll}\text { Bibliography } & 175\end{array}$

$\begin{array}{ll}\text { Index } & 181\end{array}$ 


\section{Abstract}

Let $k$ be an extension field of $\mathbb{Q}$ which is algebraically closed and has characteristic zero, and $k(x)[\partial]$ be the ring of differential operators with coefficients in $k(x)$. Let $\mathrm{L} \in k(x)[\partial]$ be an irreducible third-order linear differential operator without Liouvillian solutions. Let $\mathrm{E}=\left\{\check{\mathrm{B}}_{\nu}^{2},{ }_{1} F_{1}^{2},{ }_{0} F_{2},{ }_{1} F_{2},{ }_{2} F_{2}\right\}$ where $\mathrm{B}_{\nu}$ is the Bessel function (see [2]), $\check{\mathrm{B}}_{\nu}^{2}(x)=\mathrm{B}_{\nu}^{2}(\sqrt{x})$ and ${ }_{p} F_{q}$ with $p \in\{0,1,2\}, q \in\{1,2\}$, the generalized hypergeometric functions (see [40] and [29]). The goal of this thesis is to find a solution (if that exists) of $\mathrm{L}$ in terms of $S \in \mathrm{E}$, change of variables, algebraic operations and exponential integrals. That means to find a solution (if it exists) of the form

$$
\exp \left(\int r d x\right)\left(r_{0} S(f(x))+r_{1}(S(f(x)))^{\prime}+r_{2}(S(f(x)))^{\prime \prime}\right)
$$

where $r, r_{0}, r_{1}, r_{2}, f \in k(x)$. We have implemented in Maple five solvers for $\check{\mathrm{B}}_{\nu}^{2},{ }_{1} F_{1}^{2},{ }_{0} F_{2}$, ${ }_{1} F_{2}$ and ${ }_{2} F_{2}$. We complete the work by providing explicit examples for each solver. 


\section{List of abbreviations}

deg: degree.

denom: denominator.

gcd: greatest common divisor.

lcm: least common multiple.

numer: numerator.

$\mathbb{N}=$ set of natural numbers with zero included.

$\mathbb{N}^{*}=$ set of natural numbers without zero included.

$\mathbb{Q}=$ set of rational numbers.

$\mathbb{R}=$ set of real numbers.

$\mathbb{C}=$ set of complex numbers.

$\mathrm{K}[x]=$ ring of polynomials with one variable $x$ and coefficients in a field $\mathrm{K}$. 


\section{Chapter 0}

\section{General Introduction}

Ordinary differential equations have always been of interest since they occur in many applications. Although there is no general algorithm to solve every equation, there are many methods, such as integrating factors, symmetry method, etc.

A special class of ordinary differential equations is the class of linear homogeneous differential equations $\mathrm{L}(y)=0$, where $\mathrm{L}$ is a linear differential operator

$$
\mathrm{L}=\sum_{i=0}^{n} a_{i} \partial^{i}
$$

with $n \in \mathbb{N}$ and the coefficients $a_{i}$ in some differential field $\mathrm{K}$, e.g. $\mathrm{K}=\mathbb{Q}(x)$ or $\mathrm{K}=\mathbb{C}(x)$ and $\partial=\frac{d}{d x}$. Information on the solutions of the differential equation $\mathrm{L}(y)=0$ can be obtained by studying algebraic properties of the operator L, see e.g. van der Put and Singer [31]. Beke's algorithm and the algorithm in [32] help us to factor L when L is reducible. This makes the operator's resolution easy. If the order of $\mathrm{L}$ is larger than two, one can look for Eulerian solutions, that are solutions which can be expressed as products of secondorder operators (using sums, products, field operations, algebraic extensions, integrals, differentiations, exponentials, logarithms and change of variables). Singer showed in [35] that solving such an operator L can be reduced to solving second-order operators through factoring operators (see [17], [16], [18] and [22]), or reducing operators to tensor products of lower order operators. For irreducible operators, we can try to find Liouvillian solutions by using Kovacic's algorithm [27].

There are some special linear differential operators which don't have Eulerian solutions, which are not reducible and which don't have Liouvillian solutions. That is the case for some differential operators coming from certain special and useful functions. For example, we have the operators coming from: the Bessel square root functions $\check{\mathrm{B}}_{\nu}^{2}(x)=\mathrm{B}_{\nu}^{2}(\sqrt{x})$ where $\mathrm{B}_{\nu}$ are the Bessel functions with parameter not in $1 / 2+\mathbb{Z}$ and the functions ${ }_{1} F_{1}^{2},{ }_{0} F_{2},{ }_{1} F_{2}$ and ${ }_{2} F_{2}$. That is why we focus on those operators in this thesis.

Let $k$ be an extension field of $\mathbb{Q}$ which is algebraically closed and has characteristic zero, and $k(x)[\partial]$ be the ring of differential operators with coefficients in $k(x)$. Let $\mathrm{L} \in k(x)[\partial]$ be an irreducible linear differential operator of order $n$ without Liouvillian solutions, and $S(x)$ a special function that satisfies a linear differential equation of order $n$ with $\mathrm{L}_{S}$ as its associated operator.

Definition 0.1. A function $y$ is called a linear S-expression if there exist algebraic func- 
tions $f, r, r_{0}, r_{1}, \ldots, r_{n-1}$ such that

$y=\exp \left(\int r d x\right)\left(r_{0} S(f(x))+r_{1}(S(f(x)))^{(1)}+r_{2}(S(f(x)))^{(2)}+\cdots+r_{n-1}(S(f(x)))^{(n-1)}\right)$

We say that $y$ can be expressed in terms of $S$.

The form (1) is closed under the three following transformations that send irreducible order $n$ operators in $k(x)[\partial]$ to linear order $n$ irreducible operators:

(i) change of variables: $y(x) \rightarrow y(f(x)), \quad f \in k(x) \backslash k$,

(ii) exp-product: $y \rightarrow \exp \left(\int r d x\right) y, r \in k(x)$, and

(iii) gauge transformation: $y \rightarrow r_{0} y+r_{1} y^{\prime}+\cdots+r_{n-1} y^{(n-1)}, \quad r_{0}, r_{1}, \ldots, r_{n-1} \in k(x)$.

where $y^{(j)}$ represents the $j^{\text {th }}$ derivative of $y$ according to the variable $x$. The function $f$ in (i) above is called pullback function. These transformations are denoted by $\stackrel{f}{\longrightarrow}, \stackrel{r}{\longrightarrow}$, $\stackrel{r_{0}, r_{1}, \ldots, r_{n-1}}{\longrightarrow} G$ respectively. Hence, finding a solution $y$ of $\mathrm{L}$ in terms of $S$ corresponds to finding a sequence of those transformations that sends $\mathrm{L}_{S}$ to L. So every complete solver for finding solutions in terms of $S$ must be able to deal with all those transformations, i.e. it must be able to find any solution of the form (1) if it exists.

Let $\mathrm{E}=\left\{\check{\mathrm{B}}_{\nu}^{2},{ }_{1} F_{1}^{2},{ }_{0} F_{2},{ }_{1} F_{2},{ }_{2} F_{2}\right\}$ where $\check{\mathrm{B}}_{\nu}^{2}(x)=\mathrm{B}_{\nu}^{2}(\sqrt{x})$ with $\mathrm{B}_{\nu}$ the Bessel function (see [2]), and ${ }_{p} F_{q}$ with $p \in\{0,1,2\}, q \in\{1,2\}$, the generalized hypergeometric functions (see [40] and [29]). We are interested here in the case of order $n=3$ such that $y$ in the definition above can be a solution of our given operator $\mathrm{L}$ with $S \in \mathrm{E}$. The goal of this thesis is the following: given $\mathrm{L} \in k(x)[\partial]$, an irreducible third-order linear differential operator without Liouvillian solutions and $S \in \mathrm{E}$ with $\mathrm{L}_{S}$ as its associated differential operator, our task is to find

$$
\mathrm{L}_{S} \stackrel{f}{\longrightarrow} C \mathrm{M} \stackrel{r}{\longrightarrow} \mathrm{L}_{1}{\stackrel{r_{0}, r_{1}, r_{2}}{\longrightarrow}}_{G} \mathrm{~L}
$$

with $r, r_{0}, r_{1}, r_{2}, f \in k(x)$ and $\mathrm{M}, \mathrm{L}_{1} \in k(x)[\partial]$. A solution $y$ of $\mathrm{L}$ in terms of $S$ will be

$$
y=\exp \left(\int r d x\right)\left(r_{0} S(f(x))+r_{1}(S(f(x)))^{\prime}+r_{2}(S(f(x)))^{\prime \prime}\right) .
$$

We compute those transformations $\left(r, r_{0}, r_{1}, r_{2}, f\right)$ from the singularities of $\mathrm{M}$ (see Definition 1.12) which are not apparent (see Definition 1.15). Since we don't yet know $\mathrm{M}$, the only singularities of $\mathrm{M}$ that we know are those singularities of $\mathrm{M}$ that cannot disappear (turn into regular points (see Definition 1.12)) under transformations $\stackrel{r_{0}, r_{1}, r_{2}}{\longrightarrow}{ }_{G}$ and $\stackrel{r}{\longrightarrow}_{E}$.

Definition 0.2. A singularity of an operator is called non-removable if it stays singular under any combination of $\stackrel{r_{0}, r_{1}, r_{2}}{\longrightarrow}$ and $\stackrel{r}{\longrightarrow}{ }_{E}$. Otherwise, it is called removable singularity.

In order to find $f$, our approach is the following:

1. For $S \in\left\{{ }_{1} F_{1}^{2},{ }_{2} F_{2}\right\}$, the ramification index (see [32] and [16]) of $\mathrm{L}_{S}$ at $\infty$ is 1 . Hence, we can compute the polar part of $f$ (see Definition 3.26) from the generalized exponents (see also [32] and [16]) at irregular singularities of L (see Definition 1.15), and then $f$ by using the regular singularities of $\mathrm{L}$ (see Definition 1.15) or some information related to the degree of the numerator that $f$ can have. 
2. Let us denote by $\mathrm{L}_{B_{\nu}^{2}}$ the operator associated to the Bessel square function $\mathrm{B}_{\nu}^{2}$. For $S=\check{\mathrm{B}}_{\nu}^{2}=\mathrm{B}_{\nu}^{2}(\sqrt{x})$, the operator $\mathrm{L}_{S}=\mathrm{L}_{\check{B}_{\nu}^{2}}$ can be derived from $\mathrm{L}_{B_{\nu}^{2}}$ by the transformation $\sqrt{x}$. Hence, if $f=g^{2}$ with $g \in k(x)$, then the transformation

$$
\mathrm{L}_{\check{B}_{\nu}^{2}} \stackrel{f}{\longrightarrow} C \mathrm{M}
$$

is equivalent to the transformation

$$
\mathrm{L}_{B_{\nu}^{2}} \stackrel{g}{\longrightarrow} C \mathrm{M}
$$

Since the ramification index of $\mathrm{L}_{B_{\nu}^{2}}$ at $\infty$ is 1 , we can use, when $f=g^{2}$ with $g \in k(x)$, the same approach as in 1 . above to find $g$, and then $f$.

3. For $S \in\left\{\check{\mathrm{B}}_{\nu}^{2},{ }_{0} F_{2},{ }_{1} F_{2}\right\}$, the ramification index is $n_{e} \geq 2\left(n_{e}=2\right.$ for $\check{\mathrm{B}}_{\nu}^{2}$ when $f \neq g^{2}$ with $g \in k(x)$ or for ${ }_{1} F_{2}$, and $n_{e}=3$ for $\left.{ }_{0} F_{2}\right)$. We put $f$ in the form $f=\frac{\mathrm{A}}{\mathrm{B}}$ with $\mathrm{A}, \mathrm{B} \in k[x], \mathrm{B}$ monic and $\operatorname{gcd}(\mathrm{A}, \mathrm{B})=1$. Using the generalized exponents at the irregular singularities of L (see [42]), we can compute B and a bound for the degree of A. Hence, we can get the truncated series for $f$ and some linear equations for the coefficients of $\mathrm{A}$. By comparing the number of linear equations for the coefficients of $\mathrm{A}$ and the degree of $\mathrm{A}$, we will deal with some cases which will help us to find $\mathrm{A}$.

Since finding $f$ is equivalent to finding M, we also get M. Our basic strategy to find $r, r_{0}, r_{1}$ and $r_{2}$ is to use the $p$-curvature test (see [24], [30], [10] and [4]) between $\mathrm{M}$ and $\mathrm{L}$, and some information coming from the generalized exponents of $\mathrm{L}$ and $\mathrm{M}$ at their non-removable singularities.

This thesis is organized as follows: first, we will give some preliminaries about differential operators, they singularities, and an overview over Bessel and hypergeometric functions. We will also deal with formal solutions and generalized exponents. Chapter two will describe the above three transformations and how they affect the generalized exponents. In chapter three, we will clarify our problem and give the main step to solve it. Chapter four will show us how to have Bessel square root type solutions, and chapter five how to have $S$-type solutions with $S \in\left\{{ }_{1} F_{1}^{2},{ }_{0} F_{2},{ }_{1} F_{2},{ }_{2} F_{2}\right\}$. We will also handle the constant parameter $\nu$ of the Bessel function, and the upper and lower parameters of $S \in\left\{{ }_{1} F_{1}^{2},{ }_{0} F_{2},{ }_{1} F_{2},{ }_{2} F_{2}\right\}$. For any solver, we will apply the resolution algorithm developed case by case with explicit examples.

We have all our algorithms implemented in one Maple package called Solver3 which is part of this dissertation. All the examples in this thesis and others are included in a Maple worksheet and are also a part of this dissertation.

There is no publication until now which can solve those families of third-order linear differential equations that we study in this thesis. Let's just show by the following simple example on the ${ }_{1} F_{2}$ type solutions at which all other existing algorithms fail to solve. The ${ }_{1} F_{2}$ differential operator (see the first section of chapter three) that we denote by $\mathrm{L}_{12}$, is given by

$$
\mathrm{L}_{12}=x^{2} \partial^{3}+x\left(1+b_{1}+b_{2}\right) \partial^{2}+\left(b_{1} b_{2}-x\right) \partial-a_{1} .
$$

We consider this operator $\mathrm{L}_{12}$ with upper parameter $a_{1}=1 / 4$ and lower parameters $b_{1}=1 / 2$ and $b_{2}=3 / 4$ :

$$
\text { L L12: }=\operatorname{subs}(\{\mathrm{a} 1=1 / 4, \mathrm{~b} 1=1 / 2, \mathrm{~b} 2=3 / 4\}, \mathrm{L} 12) \text {; }
$$




$$
L 12:=x^{2} D x^{3}+9 / 4 x D x^{2}+(3 / 8-x) D x-1 / 4
$$

Let us apply to it the change of variables transformation with parameter $f$ :

$>f:=(x-1) /\left(x^{\wedge} 2\right)$;

That gives us the following operator called $\mathrm{L}_{1}$

$$
f:=\frac{x-1}{x^{2}}
$$

$$
\begin{aligned}
>\quad \mathrm{L} 1: & =\text { ChangeOfVariables }(\mathrm{L} 12, \mathrm{f}) \\
L 1:= & 8(x-1)^{2} x^{5}(x-2)^{2} D x^{3}+6\left(5 x^{2}-20 x+12\right)(x-1) x^{4}(x-2) D x^{2} \\
& +\left(15 x^{6}-128 x^{5}+420 x^{4}-592 x^{3}+544 x^{2}-384 x+128\right) x D x+2(x-2)^{5}
\end{aligned}
$$

Let us apply to it the exp-product transformation with parameter $r$ :

$>r:=-1 / x$;

$$
r:=-x^{-1}
$$

We get the following operator called $\mathrm{L}_{2}$

$>$ L2:=ExpProduct $(\mathrm{L} 1, \mathrm{r})$;

$$
\begin{aligned}
L 2:= & 8(x-1)^{2} x^{5}(x-2)^{2} D x^{3}+6\left(9 x^{2}-32 x+20\right)(x-1) x^{4}(x-2) D x^{2} \\
& +\left(75 x^{6}-548 x^{5}+1404 x^{4}-1504 x^{3}+832 x^{2}-384 x+128\right) x D x \\
& +15 x^{6}-126 x^{5}+400 x^{4}-512 x^{3}+384 x^{2}-224 x+64
\end{aligned}
$$

Finally, let us apply to $\mathrm{L}_{2}$ the gauge transformation with parameters:

$$
\begin{array}{ll}
>\mathrm{r} 0:=2 * \mathrm{x} ; & r 0:=2 x \\
>\mathrm{r} 1:=\mathrm{x} \wedge 2 ; & r 1:=x^{2} \\
>\mathrm{r} 2:=0 ; & r 2:=0
\end{array}
$$

That gives us the following operator called L that we will consider as our given operator:

$$
\begin{aligned}
> & \mathrm{L}:=\text { GaugeTransf }(\mathrm{L} 2, \mathrm{r} 0, \mathrm{r} 1, \mathrm{r} 2) ; \\
L:= & 8 x^{5}(x-2)^{2}\left(3 x^{6}+2 x^{5}+80 x^{4}-336 x^{3}+608 x^{2}-544 x+192\right)^{2}(x-1)^{5} D x^{3} \\
& +2 x^{4}(x-2)\left(3 x^{6}+2 x^{5}+80 x^{4}-336 x^{3}+608 x^{2}-544 x+192\right)\left(45 x^{8}+1756 x^{6}\right. \\
& \left.-70 x^{7}-13848 x^{5}+44640 x^{4}-79520 x^{3}+81600 x^{2}-44672 x+9984\right)(x-1)^{4} D x^{2} \\
& +x(x-2)\left(3 x^{6}+2 x^{5}+80 x^{4}-336 x^{3}+608 x^{2}-544 x+192\right)\left(45 x^{11}-54 x^{10}\right. \\
& +1976 x^{9}-22440 x^{8}+100336 x^{7}-257888 x^{6}+415872 x^{5}-437632 x^{4}+318464 x^{3} \\
& \left.-172032 x^{2}+65536 x-12288\right)(x-1)^{3} D x+32\left(+11 x^{5}-33 x^{4}+68 x^{3}-94 x^{2}\right. \\
& \left.+2 x^{6}+72 x-24\right)\left(3 x^{6}+2 x^{5}+80 x^{4}-336 x^{3}+608 x^{2}-544 x+192\right)(x-1)^{3} \\
& \times(x-2)^{4}
\end{aligned}
$$

There are no implemented algorithms that can take this operator $\mathrm{L}$ as an input value and returns its solutions in terms of ${ }_{1} F_{2}$ hypergeometric functions. Let us try for example the Maple dsolve command:

$>$ dsolve(diffop2de(L,y (x)),y(x)); 


$$
\begin{aligned}
y(x)= & \text { DESol }\left(\left\{\left(64 x^{9}-32 x^{8}-2400 x^{7}+12224 x^{6}-31552 x^{5}+54912 x^{4}-68096 x^{3}\right.\right.\right. \\
& \left.+56320 x^{2}-27648 x+6144\right)-Y(x)+\left(45 x^{12}-54 x^{11}+1976 x^{10}-22440 x^{9}\right. \\
& +100336 x^{8}-257888 x^{7}+415872 x^{6}-437632 x^{5}+318464 x^{4}-172032 x^{3} \\
& \left.+65536 x^{2}-12288 x\right) \frac{d}{d x}-Y(x)+\left(90 x^{13}-230 x^{12}+3652 x^{11}-31208 x^{10}\right. \\
& \left.+116976 x^{9}-248320 x^{8}+322240 x^{7}-252544 x^{6}+109312 x^{5}-19968 x^{4}\right) \\
& \times \frac{d^{2}}{d x^{2}}-Y(x)+\left(24 x^{14}-80 x^{13}+696 x^{12}-5216 x^{11}+18784 x^{10}-38528 x^{9}\right. \\
& \left.\left.\left.+48640 x^{8}-37632 x^{7}+16384 x^{6}-3072 x^{5}\right) \frac{d^{3}}{d x^{3}}-Y(x)\right\},\left\{{ }_{-} Y(x)\right\}\right)
\end{aligned}
$$

Therefore, Maple cannot find any solution of this third-order differential equation. Also by trying with Mathematica, we didn't succeed to find any solution. But one of our implemented codes called Hyp1F2Solutions can do it. That means it will return the Bessel square parameter, the exp-product parameter and also the gauge parameters that we considered at the beginning. The result of this code is the following:

$>$ Hyp1F2Solutions (L);

$>$ time ();

$$
\left.\left\{\left[\left\{\left[\frac{1}{4}\right],\left[\frac{1}{2}, \frac{3}{4}\right],\left[-\frac{1}{x}\right],\left[x^{2} D x+2 x\right]\right]\right\}, \frac{x-1}{x^{2}}\right]\right\}
$$

1.141

From this output, we observe that the hypergeometric function ${ }_{1} F_{2}$ comes with upper parameter $a_{1}=1 / 4$ and lower parameters $b_{1}=1 / 2$ and $b_{2}=3 / 4$, associated with the considered transformation parameters: $r=-1 / x, r_{0}=2 x, r_{1}=x^{2}, r_{2}=0$ and $f=(x-7) / x^{2}$. Hence, by using equation (3), we have found the following solution of $\mathrm{L}$ in terms of the ${ }_{1} F_{2}$ hypergeometric functions:

$$
\begin{aligned}
& >\quad y:=\operatorname{expand}(\exp (\operatorname{int}(\mathrm{r}, \mathrm{x})) *(\mathrm{r} 0 * \operatorname{hypergeom}([\mathrm{a} 1],[\mathrm{b} 1, \mathrm{~b} 2], \mathrm{f}) \\
& +\mathrm{r} 1 * \operatorname{normal}(\operatorname{diff}(\operatorname{hypergeom}([\mathrm{a} 1],[\mathrm{b} 1, \mathrm{~b} 2], \mathrm{f}), \mathrm{x})) \\
& +\mathrm{r} 2 * \text { normal }(\operatorname{diff}(\operatorname{hypergeom}([\mathrm{a} 1],[\mathrm{b} 1, \mathrm{~b} 2], \mathrm{f}), \mathrm{x} \$ 2)))) \\
& y:=2{ }_{1} \mathrm{~F}_{2}\left(\frac{1}{4} ; \frac{1}{2}, \frac{3}{4} ; \frac{x-1}{x^{2}}\right)-\frac{2}{3 x}{ }_{1} \mathrm{~F}_{2}\left(\frac{5}{4} ; \frac{3}{2}, \frac{7}{4} ; \frac{x-1}{x^{2}}\right) \\
& \quad+\frac{4}{3 x^{2}}{ }_{1} \mathrm{~F}_{2}\left(\frac{5}{4} ; \frac{3}{2}, \frac{7}{4} ; \frac{x-1}{x^{2}}\right)
\end{aligned}
$$




\section{Chapter 1}

\section{Preliminaries}

In this chapter, we recall some known results about differential operators, singularities, hypergeometric series, Bessel functions and operators, and formal solutions. At some places we try to improve or to give a new approach. Those results are mainly from Ruben Debeerst [11], Quan Yuan [42] and van der Put and Singer [32]. They also refer to some references like: [2], [1], [6], [12], [8], [14], [15], [34], [36], [37], [38], [31], [21], [40], [9], [13], [26], [23] and [41].

\subsection{Differential Operators and Singularities}

\subsubsection{Differential Operators}

Definition 1.1. Let $\mathrm{K}$ be a field. A derivation $\mathrm{D}$ on $\mathrm{K}$ is a linear map $\mathrm{D}: \mathrm{K} \rightarrow \mathrm{K}$ satisfying the product rule

$$
\mathrm{D}(a b)=a \mathrm{D}(b)+b \mathrm{D}(a), \quad \forall a, b \in \mathrm{K} .
$$

A field $\mathrm{K}$ with a derivation $\mathrm{D}$ is called differential field.

Theorem 1.2. Let $\mathrm{K}$ be a differential field with derivation $\mathrm{D}$, then

$$
\mathrm{C}_{\mathrm{K}}:=\{a \in \mathrm{K} \mid \mathrm{D}(a)=0\}
$$

is also a field. It is called the constant field of $\mathrm{K}$.

Proof. The proof is simple and can be found in [32].

Example 1.3. Let us assume that $\mathrm{C}_{\mathrm{K}}$ is an extension field of $\mathbb{Q}$, and $\mathrm{D}=\partial:=\frac{d}{d x}$, then

- $\mathrm{C}_{\mathrm{K}}(x)$ is a differential field, called the field of rational functions over $\mathrm{C}_{\mathrm{K}}$;

- $\mathrm{C}_{\mathrm{K}}((x))$ is a differential field, called the field of formal Laurent series over $\mathrm{C}_{\mathrm{K}}$.

In our context we will consider rational functions in terms of the variable $x$ with the "usual" derivation $\partial:=\frac{d}{d x}$. Another derivation which is sometimes used is $\partial:=x \frac{d}{d x}$.

Definition 1.4. Let $\mathrm{K}$ be a differential field with derivation $\partial$, then

$$
\mathrm{L}=\sum_{i=0}^{n} a_{i} \partial^{i}, \quad a_{i} \in \mathrm{K}
$$


is called differential operator. If $a_{n} \neq 0$, then $n$ is the degree of $\mathrm{L}$, denoted by $\operatorname{deg}(\mathrm{L})$, and $a_{n}$ is the leading coefficient of $\mathrm{L}$. In case $\mathrm{L}=0$ we define the order to be $-\infty$.

The associated ring of differential operators with coefficients in $\mathrm{K}$ is denoted by $\mathrm{K}[\partial]$.

Remarks 1.5. 1. In general, there exists $a \in \mathrm{K}$ with $\partial(a) \neq 0$. So in general, the ring $\mathrm{K}[\partial]$ is not commutative. For example when $\mathrm{K}=\mathbb{C}(x)$ and $\partial:=\frac{d}{d x}$, we have $\partial(x)=1 \neq 0$. Therefore,

$$
\partial x=x \partial+\partial(x)=x \partial+1 \neq x \partial .
$$

2. $\mathrm{K}[\partial]$ is an Euclidean ring since for $\mathrm{L}_{1}, \mathrm{~L}_{2} \in \mathrm{K}[\partial]$ with $\mathrm{L}_{1} \neq 0$, there are unique differential operators $\mathrm{Q}, \mathrm{R} \in \mathrm{K}[\partial]$ such that $\mathrm{L}_{2}=\mathrm{QL}_{1}+\mathrm{R}$ and $\operatorname{deg}(\mathrm{R})<\operatorname{deg}\left(\mathrm{L}_{1}\right)$. We call this operation right division. If $\mathrm{R}=0$ then $\mathrm{L}_{1}$ is called right divisor of $\mathrm{L}_{2}$. Since an Euclidean ring is also a principal ideal ring, we can define the greatest common right divisor and the least common right multiple of differential operators. Similarly, we can define the greatest common left divisor and the least common left multiple. But, since $\mathrm{K}[\partial]$ is not commutative, we can not define the greatest common divisor and the least common multiple of differential operators.

Every differential operator L corresponds to a homogeneous differential equation Ly $=$ 0 and vice versa. Hence, when talking about homogeneous differential equations, the term order is commonly used for the order of the corresponding operator. We will always assume that $\mathrm{L} \neq 0$.

Definition 1.6. By the solutions of a differential operator L we mean the solutions of the homogeneous linear differential equation $\mathrm{L} y=0$.

Definition 1.7. The set of all solutions of a differential operator $\mathrm{L}$ is called its solution space. It is denoted by $\mathrm{V}(\mathrm{L})$.

Remark 1.8. The set $\mathrm{V}(\mathrm{L})$ is a vector space of dimension $\operatorname{deg}(\mathrm{L})$ and a set of $\operatorname{deg}(\mathrm{L})$ linearly independent solutions of $\mathrm{L}$ is called fundamental system of $\mathrm{L}$.

Note that a linear differential equation is commonly solved by transforming it into a matrix equation of order one.

Let $k$ be a field and $\tilde{k}$ an extension field of $k$. Let us consider, for $a \in \tilde{k}$, the homomorphism

$$
\begin{aligned}
\varphi_{a}: k[X] & \longrightarrow \tilde{k} \\
P & \longmapsto P(a)
\end{aligned}
$$

and $\operatorname{Ker} \varphi_{a}=\left\{P \in k[X] \mid \varphi_{a}(P)=0\right\}$ where $k[X]$ is the ring of polynomials with unknown variable $X$ and coefficients in $k$.

Definition 1.9. We say that $a$ is algebraic over $k$ if $\operatorname{Ker} \varphi_{a} \neq\{0\}$.

The set of algebraic elements of $\tilde{k}$ over $k$ is a sub-field of $\tilde{k}$ containing $k$. We call it the algebraic closure of $k$, denoted by $\bar{k}$.

Theorem 1.10. Let $k$ be a sub-field of $\mathbb{C}$, then

$$
\overline{k((x))}=\bar{k} \cdot \bigcup_{n \in \mathbb{N}^{*}} k\left(\left(x^{1 / n}\right)\right)
$$

Proof. The proof can be found in [32]. 


\subsubsection{Singularities}

\subsubsection{Singular Points}

Let $y(x)$ be a function with values in $\mathbb{C}$.

Definition 1.11. A function $y(x)$ is called

(i) regular at $p \in \mathbb{C}$ if there exists a neighborhood $O$ of $p$ such that $y(x)$ is continuous on $O$,

(ii) regular at $\infty$ if $y\left(\frac{1}{x}\right)$ is regular at 0 ,

(iii) analytic at $p \in \mathbb{C}$ if $y(x)$ can be represented as a convergent power series

$$
y(x)=\sum_{i=0}^{\infty} a_{i}(x-p)^{i}, \quad a_{i} \in \mathbb{C} .
$$

Definition 1.12. Let $\mathrm{K}$ be a differential field, $\mathrm{C}_{\mathrm{K}}$ its constant field and $\overline{\mathrm{C}_{\mathrm{K}}}$ the algebraic closure of $\mathrm{C}_{\mathrm{K}}$. We call a point $p \in \overline{\mathrm{C}_{\mathrm{K}}} \cup\{\infty\}$ a singularity of the differential operator $\mathrm{L} \in \mathrm{K}[\partial]$, if $p$ is a zero of the leading coefficient of $\mathrm{L}$ or $p$ is a pole of one of the other coefficients. All other points are called regular.

Remark 1.13. - To understand the singularity at $x=\infty$, one can always use the change of variables $x \rightarrow \frac{1}{x}$ and deal with 0 .

- At all regular points of $\mathrm{L}$ we can find a fundamental system of power series solutions.

If $p$ is a singularity of a solution of $\mathrm{L}$, then $p$ must be a singularity of $\mathrm{L}$. But the converse is not true (see apparent singularity in the definition after this following definition).

Definition 1.14. If $p \in \overline{\mathrm{C}_{\mathrm{K}}} \cup\{\infty\}$, we define the local parameter $t_{p}$ as

$$
t_{p}=\left\{\begin{array}{cc}
x-p & \text { if } p \neq \infty \\
\frac{1}{x} & \text { if } p=\infty .
\end{array}\right.
$$

Let $\mathrm{L}_{1 / x}$ denoted the operator coming from $\mathrm{L}$ by the change of variables $x \rightarrow \frac{1}{x}$.

Definition 1.15. Let $\mathrm{L}=\sum_{i=0}^{n} a_{i} \partial^{i} \in \mathrm{K}[\partial]$ where $a_{i}$ are polynomials. A singularity $p$ of $\mathrm{L}$ is called

(i) apparent singularity if all solutions of $\mathrm{L}$ are regular at $p$,

(ii) regular singular $(p \neq \infty)$ if $t_{p}^{i} \frac{a_{n-i}}{a_{n}}$ is regular at $p$ for $1 \leq i \leq n$,

(iii) regular singular $(p=\infty)$ if $\mathrm{L}_{1 / x}$ has a regular singularity at $x=0$, and

(iv) irregular singular otherwise.

Remark 1.16. If a solution of $\mathrm{L}$ has no singularity at a point $x=p$ then $x=p$ is either a regular point or an apparent singularity or a regular singularity of $\mathrm{L}$. 


\subsection{Hypergeometric Series}

Definition 1.17. The generalized hypergeometric series ${ }_{p} F_{q}$ is defined by

$$
{ }_{p} F_{q}\left(\begin{array}{c}
\alpha_{1}, \alpha_{2}, \ldots, \alpha_{p} \\
\beta_{1}, \beta_{2}, \ldots, \beta_{q}
\end{array} \mid x\right)=\sum_{k=0}^{+\infty} \frac{\left(\alpha_{1}\right)_{k} \cdot\left(\alpha_{2}\right)_{k} \cdots\left(\alpha_{P}\right)_{k}}{\left(\beta_{1}\right)_{k} \cdot\left(\beta_{2}\right)_{k} \cdots\left(\beta_{q}\right)_{k} \cdot k !} x^{k}
$$

where $(\lambda)_{k}$ denotes the Pochhammer symbol

$$
(\lambda)_{k}:=\left\{\begin{array}{cl}
1 & \text { if } k=0, \\
\lambda \cdot(\lambda+1) \cdots(\lambda+k-1) & \text { if } k>0 .
\end{array}\right.
$$

It satisfies the following differential equation.

Theorem 1.18. The generalized hypergeometric series ${ }_{p} F_{q}$ in the previous definition satisfies the differential equation

$$
\delta\left(\delta+\beta_{1}-1\right) \cdots\left(\delta+\beta_{q}-1\right) y(x)=x\left(\delta+\alpha_{1}\right) \cdots\left(\delta+\alpha_{p}\right) y(x)
$$

where $\delta=x \frac{d}{d x}$.

Remarks 1.19. 1. For $p \leq q$ the series ${ }_{p} F_{q}$ is convergent for all $x$. For $p>q+1$ the radius of convergence is zero, and for $p=q+1$ the series converges for $|x|<1$.

2. For $p \leq q+1$ the series and its analytic continuation is called hypergeometric function.

\subsection{Bessel Functions}

\section{Bessel's Differential Equation}

The Bessel's ordinary equation of order $\nu$ with parameter $\lambda$, where $\nu, \lambda \in \mathbb{C}$, is given by

$$
t^{2} \frac{d^{2} y}{d t^{2}}+t \frac{d y}{d t}+\left(\lambda^{2} t^{2}-\nu^{2}\right) y=0
$$

By doing a change of variables from $t$ to $x$ using the substitution $x=\lambda t$ we get

$$
x^{2} \frac{d^{2} y}{d x^{2}}+x \frac{d y}{d x}+\left(x^{2}-\nu^{2}\right) y=0
$$

which is known as Bessel's differential equation of order $\nu . \nu$ is called the Bessel's parameter. The solutions of this equation are called Bessel functions of order $\nu$. This equation has singularities at 0 and $\infty$. 


\section{Bessel Functions of the first and second kind}

Let us be in the vicinity of $x=0$ with $\nu \in \mathbb{R}$. Since the equation (1.2) has a regular singular point at $x=0$, then by applying the Frobenius method we get two linear independent solutions $J_{\nu}(x)$ and $Y_{\nu}(x)$ given by

$$
\begin{aligned}
J_{\nu}(x) & = \begin{cases}\sum_{k=0}^{\infty} \frac{(-1)^{k}}{k ! \Gamma(\nu+k+1)}\left(\frac{x}{2}\right)^{\nu+2 k} & \text { for }|\nu| \neq \frac{1}{2}, \\
\sqrt{\frac{2}{\pi x}} \cos (x) & \text { for } \nu=-\frac{1}{2}, \\
\sqrt{\frac{2}{\pi x}} \sin (x) & \text { for } \nu=\frac{1}{2},\end{cases} \\
\text { and } \quad Y_{\nu}(x)=\frac{J_{\nu}(x) \cos (\pi \nu)-J_{-\nu}(x)}{\sin (\pi \nu)} & \text { if } \nu \notin \mathbb{Z} .
\end{aligned}
$$

$J_{\nu}$ is the Bessel function of the first kind and $Y_{\nu}$ the Bessel function of the second kind (Neumann function or Weber function). Since Bessel's equation has no finite singular points except the origin, the series

$$
\sum_{k=0}^{+\infty} \frac{(-1)^{k}}{k ! \Gamma(\nu+k+1)}\left(\frac{x}{2}\right)^{\nu+2 k},
$$

converges for all finite $x \neq 0$. By the d'Alembert criteria we have the convergence for all finite $x$ if $\nu \geq 0$. It is clear that if $\nu$ is not an integer $Y_{\nu}(x)$ must be a solution of (1.2), but if not, it will be like an undefined form since $\cos (\pi \nu)=(-1)^{\nu}$ and $\sin (\pi \nu)=0$. Let $m \in \mathbb{Z}$, by the application of Hospital's rule:

$$
\begin{aligned}
Y_{m}(x)= & \lim _{\nu \rightarrow m} \frac{-\pi \sin \pi \nu J_{\nu}(x)+\cos (\pi \nu) J_{\nu}(x)^{\prime}-J_{-\nu}(x)^{\prime}}{\pi \cos (\pi \nu)} \\
= & \frac{1}{\pi}\left[\lim _{\nu \rightarrow m} J_{\nu}(x)^{\prime}\right]-(-1)^{m} \frac{1}{\pi}\left[\lim _{\nu \rightarrow m} J_{-\nu}(x)^{\prime}\right] \\
= & \frac{2}{\pi} J_{m}(x) \log \left(\frac{x}{2}\right)-\frac{1}{\pi} \sum_{k=0}^{m-1} \frac{(m-k-1) !}{k !}\left(\frac{x}{2}\right)^{2 k-m} \\
& \quad-\frac{1}{\pi} \sum_{k=0}^{+\infty} \frac{(-1)^{k}}{(k+m) ! k !}[\psi(m+k+1)-\psi(k+1)]\left(\frac{x}{2}\right)^{2 k+m} .
\end{aligned}
$$

where $\psi$ is the Digamma function given by relation

$$
\frac{\Gamma^{\prime}(\nu+k+1)}{\Gamma(\nu+k+1)}=\psi(\nu+k+1) .
$$

The Bessel function $J_{\nu}(x)$ can be expressed in terms of hypergeometric functions

$$
J_{\nu}(x)=\left(\frac{x}{2}\right)^{\nu} \frac{1}{\Gamma(\nu+1)}{ }_{0} F_{1}\left(\begin{array}{c|c}
- & -\frac{1}{4} x^{2} \\
\nu+1
\end{array}\right) .
$$

\section{Modified Bessel Functions of the first and second kind}

Definition 1.20. The modified Bessel equation of order $\nu \in \mathbb{C}$ is the differential equation:

$$
x^{2} y^{\prime \prime}+x y^{\prime}-\left(x^{2}+\nu^{2}\right) y=0
$$

obtained by replacing $x$ by $i x\left(\right.$ where $\left.i^{2}=-1\right)$ in the Bessel equation of order $\nu$. 
As in Bessel's differential equation, we have two linearly independent solutions

$$
\begin{aligned}
I_{\nu}(x) & =\sum_{k=0}^{+\infty} \frac{1}{k ! \Gamma(\nu+k+1)}\left(\frac{x}{2}\right)^{\nu+2 k} \\
\text { and } \quad K_{\nu}(x) & =\frac{\pi\left(I_{-\nu}(x)-I_{\nu}(x)\right)}{2 \sin (\nu \pi)}
\end{aligned}
$$

which are called modified Bessel functions of first and second kind, respectively.

The Bessel function $I_{\nu}(x)$ can be expressed in terms of hypergeometric functions

$$
I_{\nu}(x)=\left(\frac{x}{2}\right)^{\nu} \frac{1}{\Gamma(\nu+1)}{ }^{0} F_{1}\left(\begin{array}{c|l}
- & \frac{1}{4} x^{2} \\
\nu+1
\end{array}\right) .
$$

\section{Zeroes of Bessel Functions}

The zeroes of Bessel functions are of great importance in many applications. Bessel functions of the first and second kind have an infinite number of zeroes as the values of $x$ tend to $\infty$.

The modified Bessel functions of the first kind $\left(I_{\nu}(x)\right)$ have only one zero at the point $x=0$, and the modified Bessel functions of the second kind $\left(K_{\nu}(x)\right)$ do not have zeroes. Notation: $\mathrm{B}_{\nu}$ refers to any element of $\left\{J_{\nu}, Y_{\nu}, I_{\nu}, K_{\nu}\right\}$. For example, the following lemma holds for all four elements:

Lemma 1.21. Consider $S:=\mathbb{C}(x) B_{\nu}+\mathbb{C}(x) B_{\nu}^{\prime}$, where $B_{\nu}^{\prime}=\frac{d}{d x} B_{\nu}$. The space $S$ is invariant under the substitutions $\nu \rightarrow \nu+1$ and $\nu \rightarrow-\nu$.

Proof. See Corollary 1.23 in [11].

\subsubsection{Bessel Operators}

Definition 1.22. Let $\nu \in \mathbb{C}$ and $\partial:=\frac{d}{d x}$.

(i) The Bessel differential equation of order $\nu$ corresponds to the operator

$$
\mathrm{L}=x^{2} \partial^{2}+x \partial+\left(x^{2}-\nu^{2}\right)
$$

which is called the Bessel operator and denoted by $\mathrm{L}_{B_{1}}$.

(ii) The modified Bessel differential equation of order $\nu$ corresponds to the operator

$$
\mathrm{L}=x^{2} \partial^{2}+x \partial-\left(x^{2}+\nu^{2}\right)
$$

which is called the modified Bessel operator and denoted by $\mathrm{L}_{B_{2}}$.

Lemma 1.23. The Bessel functions with parameter $\nu \in \frac{1}{2}+\mathbb{Z}$ are hyperexponential functions and in that case $\mathrm{L}_{B_{1}}$ and $\mathrm{L}_{B_{2}}$ are reducible.

Since we only consider irreducible operators, we will exclude the case $\nu \in \frac{1}{2}+\mathbb{Z}$ from this thesis. 


\subsection{Formal Solutions}

\section{Universal Extension and Generalized Exponents}

In this section, we introduce the idea of generalized exponents. Generally, the generalized exponents give us the asymptotic local information about solutions. In this section we consider operators in $\mathbb{C}((x))[\partial]$. Since we work with solutions of differential operators we have to be sure that we can have all of them; that is why we have to construct the universal Picard-Vessiot ring of $\mathbb{C}((x))$ that contains a fundamental system of solution of differential operators. Before that, let us give the general definition of the universal extension $U$ (universal Picard-Vessiot ring) of a differential field.

Let $\mathrm{K}$ be a differential field, with $\mathrm{C}_{\mathrm{K}}$ as its field of constants.

Definition 1.24. A universal extension $U$ of $\mathrm{K}$ is a minimal (simple) differential ring in which every operator $\mathrm{L} \in \mathrm{K}[\partial]$ has precisely $\operatorname{deg}(\mathrm{L}) \overline{\mathrm{C}_{\mathrm{K}}}$-linear independent solutions $\overline{\left(\mathrm{C}_{\mathrm{K}}\right.}$ the algebraic closure of $\mathrm{C}_{\mathrm{K}}$ ). It exists if $\mathrm{K}$ has an algebraically closed field $\mathrm{C}_{\mathrm{K}}$ of constants of characteristic zero.

Hence the universal extension $U$ of $\mathbb{C}((x))$ exists and for every nonzero operator $\mathrm{L} \in$ $\mathbb{C}((x))[\partial]$, and we define the solution space of $\mathrm{L}$, which has dimension $\operatorname{deg}(\mathrm{L})$, as follows

$$
\mathrm{V}(\mathrm{L})=\{y \in U \mid \mathrm{L}(y)=0\} .
$$

From now, we will take $\mathrm{K}=\mathbb{C}((x))$, i.e. $\mathrm{C}_{\mathrm{K}}=\mathbb{C}$.

At the point $x=0$ we have the following construction of a universal extension $U$ of $\mathbb{C}((x))$.

First we denote $\Omega=\bigcup_{m \in \mathbb{N}^{*}} x^{-1 / m} \mathbb{C}\left[x^{-1 / m}\right], M \subset \mathbb{C}$ such that $M \oplus \mathbb{Q}=\mathbb{C}$, and $\overline{\mathbb{C}((x))}$ the algebraic closure of $\mathbb{C}((x))$ given by $\overline{\mathbb{C}((x))}=\bigcup_{n \in \mathbb{N}^{*}} \mathbb{C}\left(\left(x^{1 / n}\right)\right)$ (see Theorem 1.10).

Theorem 1.25. 1- Define the ring $R=\overline{\mathbb{C}((x))}\left[\left\{X^{a}\right\}_{a \in M},\{E(q)\}_{q \in \Omega}, l\right]$ as the polynomial ring over $\mathbb{C}((x))$ in the infinite collection of variables $\left\{X^{a}\right\}_{a \in M} \bigcup\{E(q)\}_{q \in \Omega} \bigcup\{l\}$.

2- Define the differentiation $\delta$ on $R$ by: $\delta$ is $x \frac{d}{d x}$ on $\overline{\mathbb{C}((x))}, \delta X^{a}=a X^{a}, \delta E(q)=$ $q E(q)$, and $\delta l=1$. This turns $R$ into a differential ring.

3- Let $I \subset R$ denote the ideal generated by the elements

$$
X^{0}-1, X^{a+b}-X^{a} X^{b}, E(0)-1, E\left(q_{1}+q_{2}\right)-E\left(q_{1}\right) E\left(q_{2}\right) .
$$

Hence, $I$ is a differential ideal and $I \neq R$.

4- Put $U:=R / I$

then $U$ is a universal extension of $\mathbb{C}((x))$ which means:

* the constant field of $U$ is $\mathbb{C}$;

* if $\mathrm{L}$ has order $n$, then $\mathrm{V}(\mathrm{L}):=\operatorname{Ker}(\mathrm{L}: U \longrightarrow U)$ is a $\mathbb{C}$-vector space of dimension $n$.

Proof. The proof and other details of universal extension can be found in [32].

We can think of $E(q), X^{a}$ and $l$ as

$$
E(q)=\exp \left(\int \frac{q}{x} d x\right), X^{a}=\exp (a \ln (x)) \text { and } l=\ln (x)
$$

because $x \frac{d}{d x}$ acts: 
- on $E(q)$ as multiplication by $q$,

- on $X^{a}$ as multiplication by $a$, and

- on $l$ as the solution of the equation $x \frac{d y}{d x}=1$.

Hence, at $x=0$ we have:

Theorem 1.26. The universal extension $U$ of $\mathrm{K}$ is unique and has the form

$$
U=\overline{\mathrm{K}}\left[\left\{x^{a}\right\}_{a \in M},\{e(q)\}_{q \in \Omega}, l\right],
$$

where $M \subset \mathbb{C}$ is such that $M \oplus \mathbb{Q}=\mathbb{C}, \Omega=\bigcup_{m \geq 1} x^{-1 / m} \mathbb{C}\left[x^{-1 / m}\right], l$ the solution of the equation $x \frac{d y}{d x}=1$, and the following rules hold:

(i) The only relations between the symbols are $x^{0}=1, x^{a+b}=x^{a} x^{b}, e(0)=1$ and $e\left(q_{1}+q_{2}\right)=e\left(q_{1}\right) e\left(q_{2}\right)$.

(ii) The differentiation in $U$ is given by $\delta x^{a}=a x^{a}, \delta e(q)=q e(q)$ and $\delta l=1$ where $\delta=x \frac{d}{d x}$.

Proof. To give a complete proof we would have to introduce too many details about differential rings. This is why we refer to [32] where we can find the proof.

A solution whose formal representation in the universal extension $U$ involves $l=\ln (x)$ is called logarithmic solution.

A more detailed structure of the universal extension is given by the following lemma.

Lemma 1.27. The universal extension $U$ of $\mathbb{C}((x))$ is a $\mathbb{C}((x))[\partial]$-module which can be written as a direct sum of $\mathbb{C}((x))[\partial]$-module:

$$
\begin{aligned}
U & =\bigoplus_{q \in \Omega} e(q) \overline{\mathbb{C}((x))}\left[\left\{x^{a}\right\}_{a \in \mathbb{C} \backslash \mathbb{Q}}, l\right]=\bigoplus_{q \in \Omega} \bigoplus_{a \in \mathbb{C} /\left(\frac{1}{r_{q}} \mathbb{Z}\right)} e(q) x^{a} \mathbb{C}\left(\left(x^{1 / r_{q}}\right)\right)[l] \\
& =\bigoplus_{q \in \Omega} \bigoplus_{a \in \mathbb{C} /\left(\frac{1}{r_{q}} \mathbb{Z}\right)} e(q+a) \mathbb{C}\left(\left(x^{1 / r_{q}}\right)\right)[l]=\bigoplus_{q \in \Omega} \bigoplus_{a \in \mathbb{C} /\left(\frac{1}{r_{q}} \mathbb{Z}\right)} e(q+a) \mathbb{C}\left(\left(x^{1 / r_{q+a}}\right)\right)[l] \\
& =\bigoplus_{q \in \mathrm{E}} e(q) \mathbb{C}\left(\left(x^{1 / r_{q}}\right)\right)[l],
\end{aligned}
$$

where $\Omega=\bigcup_{m \in \mathbb{N}^{*}} x^{-1 / m} \mathbb{C}\left[x^{-1 / m}\right], \mathrm{E}=\left\{q+a \mid q \in \Omega, a \in \mathbb{C} /\left(\frac{1}{r_{q}} \mathbb{Z}\right)\right\} \subseteq \bigcup_{m \in \mathbb{N}^{*}} \mathbb{C}\left[x^{-1 / m}\right]$ and $r_{q}$ is the ramification index of $q$, i.e. the smallest number such that $q \in \mathbb{C}\left[x^{-1 / r_{q}}\right]$.

Proof. The first equality is proven in [32, Chapter 3.2], for the second we refer to [16, Chapter 2.8], and the third comes by assuming $e(q)=\exp \left(\int \frac{q}{x} d x\right)$. To have the fourth equality we use the definition of the ramification index which helps us to understand the fact that $q$ and $q+a$ (with $a \in \mathbb{C}$ ) have the same ramification index. The fifth equality is just the definition of the set $\mathrm{E}$.

Remark 1.28. Let the conditions of Lemma 1.27 be satisfied and $\mathrm{L} \in \mathbb{C}(x)[\partial]$. For $q \in \mathrm{E}$, let $R_{q}:=e(q) \mathbb{C}\left(\left(x^{1 / r_{q}}\right)\right)[l]$, then $U=\bigoplus_{q \in \mathrm{E}} R_{q}$. Put $\mathrm{V}(\mathrm{L})_{q}=\mathrm{V}(\mathrm{L}) \cap R_{q}$; since the action of $\mathrm{L}$ on $U$ leaves each $R_{q}$ invariant, one has $\mathrm{V}(\mathrm{L})=\bigoplus_{q \in \mathrm{E}} \mathrm{V}(\mathrm{L})_{q}$.

$$
y \in \mathrm{V}(\mathrm{L})_{q} \Longrightarrow y=\exp \left(\int \frac{q}{x} d x\right) S, \quad S \in \mathbb{C}\left(\left(x^{1 / r_{q}}\right)\right)[\ln (x)]
$$

$q \in \mathbb{C}\left[x^{-1 / r_{q}}\right]$ is what is called generalized exponent of $\mathrm{L}$ in the next definition (at $\left.x=0\right)$. 
Note that this construction of $\mathrm{U}$ at the point $x=0$ can also be performed at other points $x=p$ by replacing $x$ with the local parameter $t_{p}$ which is $t_{p}=x-p$ for a point $p \in \mathbb{C}$ and $t_{p}=\frac{1}{x}$ for $p=\infty$.

Definition 1.29. Let $\mathrm{L} \in \mathbb{C}(x)[\partial]$ and let $p$ be a point with local parameter $t_{p}$. An element $e \in \mathbb{C}\left[t_{p}{ }^{-1 / r_{e}}\right], r_{e} \in \mathbb{N}^{*}$ is called a generalized exponent of $\mathrm{L}$ at the point $p$ if there exists a formal solution of $\mathrm{L}$ of the form

$$
y(x)=\exp \left(\int \frac{e}{t_{p}} d t_{p}\right) S, \quad S \in \mathbb{C}\left(\left(t_{p}^{1 / r_{e}}\right)\right)\left[\ln \left(t_{p}\right)\right]
$$

where the constant term of the Puiseux series $S$ is non-zero. For a given solution this representation is unique and $r_{e} \in \mathbb{N}$ is called the ramification index of $e$.

The set of generalized exponents at a point $p$ is denoted by $\operatorname{gexp}(\mathrm{L}, p)$.

Similarly, we call $e$ a generalized exponent of the solution $y$ at the point $p$ if $y=y(x)$ has the representation (1.7) for some $S \in \mathbb{C}\left(\left(t_{p}{ }^{1 / r_{e}}\right)\right)\left[\ln \left(t_{p}\right)\right]$.

For a given generalized exponent there is a unique solution of the form (1.7) if we require the constant term of the series to be one.

If $e \in \mathbb{C}$ we just get a solution $x^{e} S$, in this case $e$ is called an exponent. If $r_{e}=1$, then $e$ is unramified, otherwise it is ramified.

Remarks 1.30. Since the order of $\mathrm{L}$ is $n$, at every point $p$ we have $y_{1}, \ldots, y_{n} \in \mathrm{V}(\mathrm{L})$ linear independent solutions of $\mathrm{L}$, that means at every point $p$ there are $n$ generalized exponents $e_{1}, \ldots, e_{n}$ such that the solution space $\mathrm{V}(\mathrm{L})$ is generated by the functions

$$
\exp \left(\int \frac{e_{i}}{t_{p}} d t_{p}\right) S_{i} \quad \text { with } S_{i} \in \mathbb{C}\left(\left(t_{p}^{1 / r_{e_{i}}}\right)\right)\left[\ln \left(t_{p}\right)\right] \quad \text { and } 1 \leq i \leq n .
$$

Theorem 1.31. Let $\mathrm{L} \in \mathrm{K}[\partial], n=\operatorname{deg}(\mathrm{L}), r \in \mathbb{N}^{*}$ and let $p$ be a point with local parameter $t_{p}$. Suppose that the ramification indices of the generalized exponents at $p$ divide $r$. Then there exists a basis $y_{1}, \ldots, y_{n}$ of $\mathrm{V}(\mathrm{L})$ which satisfies the condition: $\forall i \in\{1, \ldots, n\}$

$$
y_{i}=\exp \left(\int \frac{e_{i}}{t_{p}} d x\right) S_{i} \text { for some } S_{i} \in \mathbb{C}\left(\left(t_{p}^{1 / r}\right)\right)[\ln (x)]
$$

where $e_{1}, \ldots, e_{n} \in \mathbb{C}\left[t_{p}^{-1 / r}\right]$ are generalized exponents and the constant term of $S_{i}$ is non-zero.

Proof. We just use the definition of the universal extension and the fact that, for $i=1, \ldots, n$

$$
r_{e_{i}} \mid r \Longrightarrow\left\{\begin{array}{l}
e_{i} \in \mathbb{C}\left[t_{p}^{-1 / r}\right], \\
S_{i} \in \mathbb{C}\left(\left(t_{p}^{1 / r}\right)\right)\left[\ln \left(t_{p}\right)\right] .
\end{array}\right.
$$

The details can be found in [16, Chapter 4.3.3, theorem 5].

Remarks 1.32. By using the construction of the universal extension space, Lemma 1.27 and the definition of the generalized exponents, we get: 
1. For all $e_{1}, e_{2} \in \mathrm{E}, e_{1}-e_{2} \in \frac{1}{r_{e_{1}}} \mathbb{Z} \Leftrightarrow R_{e_{1}}=R_{e_{2}}$ (working modulo the ideal $I$ in Theorem 1.25). Therefore, by Theorem 1.31 the set of generalized exponents in $\mathrm{E}$ at $p$ is unique modulo $\frac{1}{r} \mathbb{Z}$ where $r$ is the product of all the ramification indices of the generalized exponents at $p$.

2. For two generalized exponents $e_{1}$ and $e_{2}$ at $p$, if $e_{1} \neq e_{2}$ modulo $\frac{1}{r_{e_{1}}} \mathbb{Z}$, their associated solution spaces belong to different sub-modules of the universal extension and they are not related.

3. For two generalized exponents $e_{1}$ and $e_{2}$ at $p$, if $e_{1}=e_{2}$ modulo $\frac{1}{r_{e_{1}}} \mathbb{Z}$, their associated solution spaces belong to the same sub-modules of the universal extension and they can be related. So we can have in certain cases a logarithmic solution at $p$.

4. Let $p$ be a point and $e_{1}, e_{2} \in \mathrm{E}$ two generalized exponents at $p$. Then $R_{e_{1}}=R_{e_{2}}$ if and only if $r_{e_{1}}=r_{e_{2}}$ and one of the following statements holds:

(a) at most one term in one of the coefficients of the series expansion of $e_{1}$ and $e_{2}$ at $p$ differ, and those terms that differ are roots of the same irreducible polynomial in $\mathbb{Q}[X]$;

(b) There exists $a \in \mathbb{Q}$ such that by doing the change of variable $T=\sqrt[r_{e}]{a(x-p)}$, i.e. $T^{r_{e_{1}}}=a(x-p)$, in $e_{1}$ and $e_{2}$ we have $e_{1}=e_{2}$.

Let $\mathrm{E}$ be as in Lemma 1.27.

Remark 1.33. For $q \in \mathrm{E}$ such that $\mathrm{V}(\mathrm{L})_{q} \neq\{0\}$ and $\mathrm{P}_{q}(x)$ the minimal polynomial with maximal degree of all the minimal polynomials coming from expressions related to the coefficients in the series expansion of $q$ such that taking any other root of this polynomial instead of the chosen one gets also $\mathrm{V}(\mathrm{L})_{q} \neq\{0\}$, we have

$$
\operatorname{dim} \mathrm{V}(\mathrm{L})_{q}=\operatorname{deg}\left(\mathrm{P}_{q}(x)\right) \cdot r_{q}
$$

where $\mathrm{V}(\mathrm{L})_{q}$ is defined as in Remark 1.28.

Lemma 1.34. Let $\mathrm{L} \in \mathbb{C}(x)[\partial]$ be a linear differential operator of order $n$, and as in Lemma 1.27 and Remark 1.28, $\Omega=\bigcup_{m \in \mathbb{N}^{*}} x^{-1 / m} \mathbb{C}\left[x^{-1 / m}\right], \mathrm{E}=\left\{q+a \mid q \in \Omega, a \in \mathbb{C} /\left(\frac{1}{r_{q}} \mathbb{Z}\right)\right\}$, $\mathrm{V}(\mathrm{L})_{q}=\mathrm{V}(\mathrm{L}) \cap R_{q}$ where $q \in \mathrm{E}, R_{q}:=e(q) \mathbb{C}\left(\left(x^{1 / r_{q}}\right)\right)[l]$ with $l=\ln (x)$ and $e(q)=$ $\exp \left(\int \frac{q}{x} d x\right)$. For $q \in \mathrm{E}$ such that $\mathrm{V}(\mathrm{L})_{q} \neq\{0\}$, and $\mathrm{P}_{q}(x)$ the minimal polynomial with maximal degree of all the minimal polynomials coming from expressions related to the coefficients in the series expansion of $q$ at $x=0$ such that taking any other root of this polynomial instead of the chosen one gets also $\mathrm{V}(\mathrm{L})_{q} \neq\{0\}$. Then $r_{q} \in\{1, \ldots, n\}$.

Proof. By using Remark 1.33 we have $\operatorname{dim} \mathrm{V}(\mathrm{L})_{q}=\operatorname{deg}\left(\mathrm{P}_{\mathrm{q}}(x)\right) \cdot r_{q}$. Remark 1.28 gives us $\mathrm{V}(\mathrm{L})=\bigoplus_{q \in \mathrm{E}} \mathrm{V}(\mathrm{L})_{q}$ and then $n=\sum_{q \in \mathrm{E}} \operatorname{deg}\left(\mathrm{P}_{q}(x)\right) \cdot r_{q}$. Since $\operatorname{deg}\left(\mathrm{P}_{q}(x)\right) \geq 1$ and $r_{q} \geq 1$ for all $q \in \mathrm{E}$, then $r_{q} \leq n$. So $r_{q} \in\{1, \ldots, n\}$. 


\section{Relations between Singularities and Generalized Exponents}

We consider here the case where the number of singularities of the differential operator is finite. Hence the singularities will be isolated.

Definition 1.35. If $p \in \mathbb{C} \cup\{\infty\}$, we define the local parameter $t_{p}$ as

$$
t_{p}=\left\{\begin{array}{cc}
z-p & \text { if } p \neq \infty \\
\frac{1}{z} & \text { if } p=\infty
\end{array}\right.
$$

Let

$$
\mathrm{L}=\partial^{n}+P_{n-1}(z) \partial^{n-1}+P_{n-2}(z) \partial^{n-2}+\cdots+P_{1}(z) \partial+P_{0}(z)
$$

where $P_{i}(z), 0 \leq i \leq n-1$ are analytic functions in some domain $\mathcal{D}$ of the $z$-plane. Suppose that $z_{0}$ is either an isolated singularity or a regular point of $\mathrm{L}$.

Definition 1.36. $y \in \mathrm{V}(\mathrm{L})$ is called a regular solution at $z_{0}$ if its generalized exponent $e$ belong to $\mathbb{C}$. In that case the ramification index of $e$ is 1 . That means

$$
y=t_{z_{0}}^{e} S, \quad \text { with } S \in \mathbb{C}\left(\left(t_{z_{0}}\right)\right)\left[\ln \left(t_{z_{0}}\right)\right] \text { and } e \in \mathbb{C} .
$$

When $e \notin \mathbb{C}, y$ is an irregular solution.

Lemma 1.37. $z_{0}$ is either a regular point or an apparent singularity or a regular singularity of $\mathrm{L}$ if $\mathrm{V}(\mathrm{L})$ has a basis at $z_{0}$ of regular solutions. Otherwise $z_{0}$ is an irregular singularity of $\mathrm{L}$.

Proof. We just use the Definition 1.15 of singularities of an operator and the Frobenius method.

By this lemma we can conclude the following:

Corollary 1.38. For a basis $\left\{y_{1}, \ldots, y_{n}\right\}$ of $\mathrm{V}(\mathrm{L})$ at $z_{0}$ with associated generalized exponents $\left\{e_{1}, \ldots, e_{n}\right\}$, we say that $z_{0}$ is either a regular point or an apparent singularity or a regular singularity of $\mathrm{L}$ if $\left\{e_{1}, \ldots, e_{n}\right\} \subset \mathbb{C}$. Otherwise $z_{0}$ is an irregular singularity of $\mathrm{L}$.

\section{Regular Solution}

The condition that the coefficients of the operator L must satisfy at $z_{0}$ to have a basis of regular solutions is the following:

$$
P_{i}(z)=\sum_{j=0}^{+\infty} p_{i, j} t_{z_{0}}^{j-(n-i)}, \quad \text { with } \quad p_{i, j} \in \mathbb{C}, 0 \leq i \leq n-1 .
$$

By taking solutions in the form $t_{z_{0}}^{e} S$, with $S \in \mathbb{C}\left(\left(t_{z_{0}}\right)\right)\left[\ln \left(t_{z_{0}}\right)\right]$ and $e \in \mathbb{C}$, and substitute them into the differential equation associated to $\mathrm{L}$ we get an indicial equation in the form

$$
\lambda(\lambda-1)(\lambda-2) \cdots(\lambda-(n-1))+\sum_{i=0}^{n-1} p_{i, 0} \lambda^{n-i-1}=0
$$

with $\lambda \in \mathbb{C}$. Generalized exponents of solutions of $\mathrm{L}$ at $z_{0}$ are solutions of this indicial equation. 
Lemma 1.39. Let us assume that $\mathrm{L}$ has at $z_{0}$ a basis of regular solutions which are not logarithmic. The generalized exponents of $\mathrm{L}$ at $z_{0}$ are non-negative integers if and only if $z_{0}$ is either a regular point or an apparent singularity of $\mathrm{L}$.

Proof. We just use the fact that at every regular point or apparent singularity of $\mathrm{L}$ the solutions are analytic.

Corollary 1.40. When $\mathrm{L}$ has at $z_{0}$ a basis of regular solutions and $z_{0}$ is not a regular point nor an apparent singularity of $\mathrm{L}$, then $z_{0}$ is a regular singularity of $\mathrm{L}$. That means there exists at least one generalized exponent of $\mathrm{L}$ at $z_{0}$ which is not in $\mathbb{N}$ when $\mathrm{L}$ doesn't have a logarithmic solution at $z_{0}$.

Corollary 1.41. If $z_{0}$ is a regular point of $\mathrm{L}$ then the generalized exponents of $\mathrm{L}$ at $z_{0}$ are $0,1,2, \ldots, n-1$.

Proof. Since all the coefficients $P_{i}(z)$ of $\mathrm{L}$ are regular at $z_{0}$, for $0 \leq i \leq n-1$, all the coefficients $p_{i, j}$ of the negative powers of $P_{i}(z)$ in equation (1.10) will be zero. That means, for $0 \leq i \leq n-1$, we will have

$$
p_{i, j}=0, \quad 0 \leq j<n-i
$$

Hence, $p_{i, 0}=0$ with $0 \leq i \leq n-1$ and our indicial equation will become

$$
\lambda(\lambda-1)(\lambda-2) \cdots(\lambda-(n-1))=0 .
$$

So $\lambda \in\{0,1,2, \ldots, n-1\}$.

\subsection{Maple Commands}

In this thesis, we will develop all of our algorithms, codes and examples with Maple. So in this section we want to introduce some commands we need in Maple.

In Maple, the DEtools package contains commands that help us to work with differential equations. The input to use this package is

$>$ with (DETools):

This following command tells Maple that we use $x$ as variable and $D x$ as derivation.

$>$ _Envdiffopdomain: $=[\mathrm{Dx}, \mathrm{x}]$ :

We will always assume that the DEtools package is loaded and the differential domain is defined by $[D x, x]$.

\subsubsection{From an operator to a differential equation and vice versa}

With the command diffop2de Maple can transform a linear differential operator to its associated differential equation. Let us take the irreducible third-order linear differential operator $\mathrm{L}_{B B}$ associated to the modified Bessel square function of the first kind $\mathrm{I}(\nu, x)^{2}$ with parameter $\nu$.

$$
\begin{array}{rl}
>\text { LBB }:=\mathrm{x}^{\wedge} 2 & * \mathrm{Dx} \wedge 3+3 * \mathrm{x} * \mathrm{Dx} \wedge 2+\left(1-4 * \mathrm{x}^{\wedge} 2-4 * \mathrm{nu}^{\wedge} 2\right) * \mathrm{Dx}-4 * \mathrm{x} ; \\
L B B & :=x^{2} D x^{3}+3 x D x^{2}+\left(1-4 x^{2}-4 \nu^{2}\right) D x-4 x
\end{array}
$$


With the command diffop2de Maple can transform a differential operator to the left hand side of its associated differential equation.

$>$ eq:=diffop $2 \operatorname{de}(\operatorname{LBB}, \mathrm{y}(\mathrm{x}))$;

$$
e q:=-4 x y(x)+\left(1-4 x^{2}-4 \nu^{2}\right) \frac{d}{d x} y(x)+3 x \frac{d^{2}}{d x^{2}} y(x)+x^{2} \frac{d^{3}}{d x^{3}} y(x)
$$

With the command de2diffop Maple can transform a linear differential homogeneous equation to its associated differential operator.

$>\operatorname{de} 2 \operatorname{diffop}(e q, \mathrm{y}(\mathrm{x}))$;

$$
x^{2} D x^{3}+3 x D x^{2}+\left(1-4 x^{2}-4 \nu^{2}\right) D x-4 x
$$

\subsubsection{Solving linear differential equations}

With the command dsolve Maple can solve linear differential equations.

$>$ dsolve (eq,y $(\mathrm{x}))$;

$$
y(x)={ }_{-} C 1(\mathrm{I}(\nu, x))^{2}+{ }_{-} C 2(\mathrm{~K}(\nu, x))^{2}+{ }_{-} C 3 \mathrm{I}(\nu, x) \mathrm{K}(\nu, x)
$$

where_C1 and _C2 are constants and, I $(\nu, x)$ and $\mathrm{K}(\nu, x)$ are respectively the modified Bessel functions of the first and second kind with parameter $\nu$.

\subsubsection{Factorization into irreducible linear differential operators}

With the command Dfactor Maple can factorize reducible linear differential operators (see [17], [16], [18] and [22]). If the parameter $\nu=1 / 2$ in the expression of $\mathrm{L}_{B B}$, then $\mathrm{L}_{B B}$ is reducible and we can factor it

$>\mathrm{L} 1:=\operatorname{subs}(\mathrm{nu}=1 / 2, \mathrm{LBB})$;

$$
L 1:=x^{2} D x^{3}+3 x D x^{2}-4 x^{2} D x-4 x
$$

$>$ DFactor $(\mathrm{L} 1)$;

$$
\left[x^{2} D x-x(-1+2 x), D x+\frac{1+2 x}{x}, D x+x^{-1}\right]
$$

\subsubsection{Generalized Exponents}

Generalized exponents can be computed in Maple with the command gen_exp, which belongs to the package DEtools. The input is an operator, a variable $t$ to express the generalized exponents and a point $x=p$ at which we want to compute the generalized exponents. The output is a list of pairs $[g, e q]$ which each represents a generalized exponent at the given point $p$. In this pair the equation $e q$ describes the variable $x-p$ which is used to express the generalized exponent $g$.

$$
\begin{aligned}
& >\quad \text { gen_exp }(\mathrm{LBB}, \mathrm{t}, \mathrm{x}=0) ; \\
& {[[0, t=x],[-2 \nu, t=x],[2 \nu, t=x]]} \\
& >\quad \text { gen_exp(LBB, } \mathrm{t}, \mathrm{x}=\inf \text { inity }) ; \\
& \quad\left[\left[1, t=x^{-1}\right],\left[2 t^{-1}+1, t=x^{-1}\right],\left[-2 t^{-1}+1, t=x^{-1}\right]\right] \\
& >\text { gen_exp }(\mathrm{LBB}, \mathrm{t}, \mathrm{x}=1) ;
\end{aligned}
$$

$$
[[0,1,2, t=x-1]]
$$




\subsubsection{Formal and logarithmic solutions}

With the command formal_sol Maple can compute the formal solutions of an operator at a point.

$>\mathrm{L} 2:=\operatorname{subs}(\mathrm{nu}=0, \mathrm{LBB})$;

$L 2:=x^{2} D x^{3}+3 x D x^{2}+\left(1-4 x^{2}\right) D x-4 x$

$>$ formal_sol $(\mathrm{L} 2, \mathrm{t}, \mathrm{x}=0)$;

$\left[\left[1 / 2(\ln (t))^{2}+\left(-1 / 4 \ln (t)+1 / 4(\ln (t))^{2}\right) t^{2}+\left(\frac{3}{64}(\ln (t))^{2}-\frac{11}{128} \ln (t)\right.\right.\right.$

$\left.+\frac{1}{32}\right) t^{4}+O\left(t^{6}\right), \ln (t)+(-1 / 4+1 / 2 \ln (t)) t^{2}+\left(\frac{3}{32} \ln (t)-\frac{11}{128}\right) t^{4}$

$\left.\left.+O\left(t^{6}\right), 1+1 / 2 t^{2}+\frac{3}{32} t^{4}+O\left(t^{6}\right), t=x\right]\right]$

$>$ formal_sol $(\mathrm{L} 2, \mathrm{t}, \mathrm{x}=1)$;

$$
\begin{gathered}
{\left[\left[1 / 2-1 / 2 t+5 / 8 t^{2}-\frac{13}{24} t^{3}+\frac{49}{96} t^{4}-\frac{7}{16} t^{5}+O\left(t^{6}\right),\right.\right.} \\
-t+t^{2}-3 / 2 t^{3}+\frac{11}{8} t^{4}-\frac{27}{20} t^{5}+O\left(t^{6}\right), \\
\left.\left.t^{2}-t^{3}+5 / 4 t^{4}-7 / 6 t^{5}+O\left(t^{6}\right), t=x-1\right]\right]
\end{gathered}
$$

If we want to know whether the operator $\mathrm{L}_{2}$ has logarithmic solutions at a point, we can also use the command formal_sol but with 'has logarithm?' as second argument.

$>$ formal_sol(L2, 'has logarithm?', $\mathrm{x}=0$ ) ;

true

> formal_sol(L2, 'has logarithm?', $\mathrm{x}=1$ ) ;

false 


\section{Chapter 2}

\section{Transformations}

In this chapter, some results are ours, and the others are our extension to third-order linear differential operators of results from Ruben Debeerst [11] and Quan Yuan [42] related to second-order linear differential operators. Those two authors also refer to some references like: [3], [7], [5], [7], [35], [28], and [20].

Our idea, in order to solve a differential operator in terms of solutions of another differential operator, starts by seeing first how they can be connected. That will help us to find the map between their solution spaces. Therefore, we will discuss the transformations that preserve third-order differential operators and how they can affect the generalized exponents of solutions of a third-order differential operator. We assume here that all of our operators are irreducible.

Let $k$ be an extension field of $\mathbb{Q}$ which is algebraically closed and has characteristic zero. Let $\mathrm{K}=k(x)$ be the field of rational functions in $x$.

\subsection{Types of Transformations}

Definition 2.1. A transformation between two differential operators $\mathrm{L}_{1}, \mathrm{~L}_{2} \in \mathrm{K}[\partial]$ is a map from the solution space $\mathrm{V}\left(\mathrm{L}_{1}\right)$ onto the solution space $\mathrm{V}\left(\mathrm{L}_{2}\right)$.

The transformation is invertible if there also exists a map from $\mathrm{V}\left(\mathrm{L}_{2}\right)$ onto $\mathrm{V}\left(\mathrm{L}_{1}\right)$. There are three known types of transformations that preserve the differential field and preserve order three. They are:

Definition 2.2. Let $\mathrm{L}_{1} \in \mathrm{K}[\partial]$ be a differential operator of degree three. For $y=y(x) \in$ $\mathrm{V}\left(\mathrm{L}_{1}\right)$ we have:

(i) change of variables: $y(x) \rightarrow y(f(x)), f \in \mathrm{K} \backslash k$,

(ii) exp-product: $y \rightarrow \exp \left(\int r d x\right) y, \quad r \in \mathrm{K}$, and

(iii) gauge transformation: $y \rightarrow r_{0} y+r_{1} y^{\prime}+r_{2} y^{\prime \prime}, \quad r_{0}, r_{1}, r_{2} \in \mathrm{K}$.

They are denoted by $\longrightarrow_{C}, \longrightarrow_{E}, \longrightarrow_{G}$ respectively and for the resulting operator $\mathrm{L}_{2} \in \mathrm{K}[\partial]$ we write $\mathrm{L}_{1} \stackrel{f}{\longrightarrow} \mathrm{L}_{2}, \mathrm{~L}_{1} \stackrel{r}{\longrightarrow} \mathrm{L}_{2}, \mathrm{~L}_{1} \stackrel{r_{0}, r_{1}, r_{2}}{\longrightarrow}{ }_{G} \mathrm{~L}_{2}$, respectively. Furthermore, we write $\mathrm{L}_{1} \longrightarrow \mathrm{L}_{2}$ if there exists a sequence of those transformations that sends $\mathrm{L}_{1}$ to $\mathrm{L}_{2}$. The rational functions $f, r, r_{0}, r_{1}$ and $r_{2}$ will be called parameters of the transformations, and in case $(i i)$ the function $\exp \left(\int r d x\right)$ is a hyperexponential function. 
Theorem 2.3. Let $\mathrm{L}_{1} \in \mathrm{K}[\partial]$ be an irreducible third-order linear differential operator. If the parameters of the transformations above are given, we can always find $\mathrm{L}_{2} \in \mathrm{K}[\partial]$ with $\operatorname{deg}\left(\mathrm{L}_{2}\right)=3$ coming from $\mathrm{L}_{1}$ by the transformations associated to those parameters.

Proof. Let $\mathrm{L}_{1}=a_{3}(x) \partial^{3}+a_{2}(x) \partial^{2}+a_{1}(x) \partial+a_{0}(x)$ be an irreducible third-order linear differential operator with coefficients in $\mathrm{K}\left(a_{3}(x) \neq 0\right)$. Let $y=y(x)$ be a solution of $\mathrm{L}_{1}$, i.e

$$
a_{3}(x) \frac{d^{3} y}{d x^{3}}+a_{2}(x) \frac{d^{2} y}{d x^{2}}+a_{1}(x) \frac{d y}{d x}+a_{0}(x) y=0
$$

Let us also assume, if it exists,

$$
\frac{d^{3} z}{d x^{3}}+b_{2}(x) \frac{d^{2} z}{d x^{2}}+b_{1}(x) \frac{d z}{d x}+b_{0}(x) z=0
$$

to be the equation coming from (2.1) after one of our three transformations. Let us sometimes use the notation' for $\frac{d}{d x}$.

a- The change of variables transformation:

Let $f \in \mathrm{K}$ and $z=y(f)$. Then

$$
\begin{aligned}
& z=y(f), \\
& \frac{d z}{d x}=\frac{d}{d x} y(f)=\left.\frac{d}{d x} y(x)\right|_{x=f} \cdot f^{\prime}(x), \\
& \frac{d^{2} z}{d x^{2}}=\frac{d^{2}}{d x^{2}} y(f)=\left.\frac{d^{2}}{d x^{2}} y(x)\right|_{x=f} \cdot\left(f^{\prime}(x)\right)^{2}+\left.\frac{d}{d x} y(x)\right|_{x=f} \cdot f^{\prime \prime}(x), \\
& \text { and } \frac{d^{3} z}{d x^{3}}=\frac{d^{3}}{d x^{3}} y(f)=\left.\frac{d^{2}}{d x^{2}} y(x)\right|_{x=f} \cdot\left[-\left.\frac{a_{2}(x)}{a_{3}(x)}\right|_{x=f} \cdot\left(f^{\prime}(x)\right)^{3}+3 f^{\prime}(x) f^{\prime \prime}(x)\right] \\
&+\left.\frac{d}{d x} y(x)\right|_{x=f} \cdot\left[-\left.\frac{a_{1}(x)}{a_{3}(x)}\right|_{x=f} \cdot\left(f^{\prime}(x)\right)^{3}+f^{\prime \prime \prime}(x)\right] \\
&-\left.\left.y(x)\right|_{x=f} \cdot \frac{a_{0}(x)}{a_{3}(x)}\right|_{x=f} \cdot\left(f^{\prime}(x)\right)^{3} \cdot
\end{aligned}
$$

We can rewrite the equation (2.2) in terms of $\left.y(x)\right|_{x=f},\left.\frac{d}{d x} y(x)\right|_{x=f}$ and $\left.\frac{d^{2}}{d x^{2}} y(x)\right|_{x=f}$ using the equations above and get

$$
\begin{aligned}
& \left.\frac{d^{2}}{d x^{2}} y(x)\right|_{x=f} \cdot\left[-\left.\frac{a_{2}(x)}{a_{3}(x)}\right|_{x=f} \cdot\left(f^{\prime}(x)\right)^{3}+b_{2}(x)\left(f^{\prime}(x)\right)^{2}+3 f^{\prime}(x) f^{\prime \prime}(x)\right] \\
& +\left.\frac{d}{d x} y(x)\right|_{x=f} \cdot\left[-\left.\frac{a_{1}(x)}{a_{3}(x)}\right|_{x=f} \cdot\left(f^{\prime}(x)\right)^{3}+b_{2}(x) f^{\prime \prime}(x)+b_{1}(x) f^{\prime}(x)+f^{\prime \prime \prime}(x)\right] \\
& +\left.y(x)\right|_{x=f} \cdot\left[-\left.\frac{a_{0}(x)}{a_{3}(x)}\right|_{x=f} \cdot\left(f^{\prime}(x)\right)^{3}+b_{0}(x)\right]=0 .
\end{aligned}
$$


Since $\mathrm{L}_{1}$ is irreducible, all the coefficients of $\left.y(x)\right|_{x=f},\left.\frac{d}{d x} y(x)\right|_{x=f}$ and $\left.\frac{d^{2}}{d x^{2}} y(x)\right|_{x=f}$ in the equation (2.3) have to be zero. That gives us:

$$
\left\{\begin{array}{l}
b_{0}(x)=\left.\frac{a_{0}(x)}{a_{3}(x)}\right|_{x=f} \cdot\left(f^{\prime}(x)\right)^{3}, \\
b_{1}(x)=3\left(\frac{f^{\prime \prime}(x)}{f^{\prime}(x)}\right)^{2}-\frac{f^{\prime \prime \prime}(x)}{f^{\prime}(x)}-\left.\frac{a_{2}(x)}{a_{3}(x)}\right|_{x=f} \cdot f^{\prime \prime}(x)+\left.\frac{a_{1}(x)}{a_{3}(x)}\right|_{x=f} \cdot\left(f^{\prime}(x)\right)^{2}, \\
b_{2}(x)=-3 \frac{f^{\prime \prime}(x)}{f^{\prime}(x)}+\left.\frac{a_{2}(x)}{a_{3}(x)}\right|_{x=f} \cdot f^{\prime}(x) .
\end{array}\right.
$$

Thus, we have found a differential equation (2.2) for $z=y(f)$ which has order three.

b- The exp-product transformation:

Let $r \in \mathrm{K}$ and $z=\exp \left(\int r d x\right) y(x)$. Then

$$
\begin{aligned}
z= & \exp \left(\int r d x\right) y(x), \\
\frac{d z}{d x}= & {\left[\frac{d y(x)}{d x}+r y(x)\right] \exp \left(\int r d x\right), } \\
\frac{d^{2} z}{d x^{2}}= & {\left[\frac{d^{2} y(x)}{d x^{2}}+2 r \frac{d y(x)}{d x}+\left(r^{\prime}+r^{2}\right) y(x)\right] \exp \left(\int r d x\right), } \\
\text { and } \frac{d^{3} z}{d x^{3}}= & {\left[\left(3 r-\frac{a_{2}(x)}{a_{3}(x)}\right) \frac{d^{2} y(x)}{d x^{2}}+\left(3 r^{2}+3 r^{\prime}-\frac{a_{1}(x)}{a_{3}(x)}\right) \frac{d y(x)}{d x}\right.} \\
& \left.+\left(r^{\prime \prime}+3 r r^{\prime}+r^{3}-\frac{a_{0}(x)}{a_{3}(x)}\right) y(x)\right] \exp \left(\int r d x\right) .
\end{aligned}
$$

We can rewrite the equation (2.2) in terms of $y(x), \frac{d y(x)}{d x}$ and $\frac{d^{2} y(x)}{d x^{2}}$ using the equations above and get

$$
\begin{aligned}
& {\left[\left(b_{2}(x)+3 r-\frac{a_{2}(x)}{a_{3}(x)}\right) \frac{d^{2} y(x)}{d x^{2}}+\left(3 r^{2}+3 r^{\prime}+2 r b_{2}(x)+b_{1}(x)-\frac{a_{1}(x)}{a_{3}(x)}\right) \frac{d y(x)}{d x}\right.} \\
+ & \left.\left(r^{\prime \prime}+3 r r^{\prime}+r^{3}+\left(r^{\prime}+r^{2}\right) b_{2}(x)+r b_{1}(x)+b_{0}(x)-\frac{a_{0}(x)}{a_{3}(x)}\right) y(x)\right] \exp \left(\int r d x\right) \\
= & 0 .
\end{aligned}
$$

Since $\mathrm{L}_{1}$ is irreducible, all the coefficients of $y(x), \frac{d y(x)}{d x}$ and $\frac{d^{2} y(x)}{d x^{2}}$ in the equation (2.4) have to be zero. That gives us:

$$
\left\{\begin{array}{l}
b_{0}(x)=\left(r^{2}-r^{\prime}\right) \frac{a_{2}(x)}{a_{3}(x)}-r \frac{a_{1}(x)}{a_{3}(x)}+\frac{a_{0}(x)}{a_{3}(x)}-r^{\prime \prime}+3 r r^{\prime}-r^{3} \\
b_{1}(x)=\frac{a_{1}(x)}{a_{3}(x)}-2 r \frac{a_{2}(x)}{a_{3}(x)}+3 r^{2}-3 r^{\prime} \\
b_{2}(x)=\frac{a_{2}(x)}{a_{3}(x)}-3 r
\end{array}\right.
$$

Thus, we have found a differential equation (2.2) for $z=\exp \left(\int r d x\right) y(x)$ which has order three. 
c- The gauge transformation:

Let $r_{0}, r_{1}, r_{2} \in \mathrm{K}$ and $z=r_{2} \frac{d^{2} y(x)}{d x^{2}}+r_{1} \frac{d y(x)}{d x}+r_{0} y(x)$. Then

$$
\begin{aligned}
z= & r_{2} \frac{d^{2} y(x)}{d x^{2}}+r_{1} \frac{d y(x)}{d x}+r_{0} y(x), \\
\frac{d z}{d x}= & \left(r_{2}^{\prime}+r_{1}-r_{2} \frac{a_{2}(x)}{a_{3}(x)}\right) \frac{d^{2} y(x)}{d x^{2}}+\left(r_{1}^{\prime}+r_{0}-r_{2} \frac{a_{1}(x)}{a_{3}(x)}\right) \frac{d y(x)}{d x} \\
& \quad+\left(r_{0}^{\prime}-r_{2} \frac{a_{0}(x)}{a_{3}(x)}\right) y(x), \\
\frac{d^{2} z}{d x^{2}}= & {\left[r_{2}\left(\frac{a_{2}(x)}{a_{3}(x)}\right)^{2}-r_{2}\left(\frac{a_{2}(x)}{a_{3}(x)}\right)^{\prime}-\left(r_{1}+2 r_{2}^{\prime}\right) \frac{a_{2}(x)}{a_{3}(x)}-r_{2} \frac{a_{1}(x)}{a_{3}(x)}+r_{2}^{\prime \prime}+2 r_{1}^{\prime}+r_{0}\right] } \\
\times & \frac{d^{2} y(x)}{d x^{2}}+\left[r_{2} \frac{a_{1}(x)}{a_{3}(x)} \cdot \frac{a_{2}(x)}{a_{3}(x)}-r_{2}\left(\frac{a_{1}(x)}{a_{3}(x)}\right)^{\prime}-\left(r_{1}+2 r_{2}^{\prime}\right) \frac{a_{1}(x)}{a_{3}(x)}+r_{1}^{\prime \prime}+2 r_{0}^{\prime}\right. \\
- & \left.r_{2} \frac{a_{0}(x)}{a_{3}(x)}\right] \frac{d y(x)}{d x}+\left[r_{2} \frac{a_{0}(x)}{a_{3}(x)} \cdot \frac{a_{2}(x)}{a_{3}(x)}-r_{2}\left(\frac{a_{0}(x)}{a_{3}(x)}\right)^{\prime}-\left(r_{1}+2 r_{2}^{\prime}\right) \frac{a_{0}(x)}{a_{3}(x)}+r_{0}^{\prime \prime}\right] \\
\times & y(x),
\end{aligned}
$$

and

$$
\begin{aligned}
\frac{d^{3} z}{d x^{3}} & =\left[2 r_{2} \frac{a_{1}(x)}{a_{3}(x)} \cdot \frac{a_{2}(x)}{a_{3}(x)}+3 r_{2} \frac{a_{2}(x)}{a_{3}(x)} \cdot\left(\frac{a_{2}(x)}{a_{3}(x)}\right)^{\prime}-r_{2}\left(\frac{a_{2}(x)}{a_{3}(x)}\right)^{\prime \prime}-r_{2}^{\prime}\left(\frac{a_{2}(x)}{a_{3}(x)}\right)^{\prime}\right. \\
& -2 r_{2}\left(\frac{a_{1}(x)}{a_{3}(x)}\right)^{\prime}-\left(2 r_{2}^{\prime}+r_{1}\right)\left(\frac{a_{2}(x)}{a_{3}(x)}\right)^{\prime}-r_{2}\left(\frac{a_{2}(x)}{a_{3}(x)}\right)^{3}+\left(3 r_{2}^{\prime}+r_{1}\right)\left(\frac{a_{2}(x)}{a_{3}(x)}\right)^{2} \\
& \left.-\left(3 r_{2}^{\prime \prime}+3 r_{1}^{\prime}+r_{0}\right) \frac{a_{2}(x)}{a_{3}(x)}-\left(3 r_{2}^{\prime}+r_{1}\right) \frac{a_{1}(x)}{a_{3}(x)}-r_{2} \frac{a_{0}(x)}{a_{3}(x)}\right] \frac{d^{2} y(x)}{d x^{2}} \\
& +\left[r_{2}\left(\frac{a_{1}(x)}{a_{3}(x)} \cdot \frac{a_{2}(x)}{a_{3}(x)}\right)^{\prime}+r_{2} \frac{a_{1}(x)}{a_{3}(x)} \cdot\left(\frac{a_{2}(x)}{a_{3}(x)}\right)^{\prime}-r_{2} \frac{a_{1}(x)}{a_{3}(x)} \cdot\left(\frac{a_{2}(x)}{a_{3}(x)}\right)^{2}\right. \\
& -r_{2}\left(3 r_{2}^{\prime}+r_{1}\right) \frac{a_{0}(x)}{a_{3}(x)} \cdot \frac{a_{2}(x)}{a_{3}(x)}+r_{2} \frac{a_{0}(x)}{a_{3}(x)} \cdot \frac{a_{2}(x)}{a_{3}(x)}+r_{2}\left(\frac{a_{1}(x)}{a_{3}(x)}\right)^{2}-r_{2}\left(\frac{a_{1}(x)}{a_{3}(x)}\right)^{\prime \prime} \\
& -r_{2}^{\prime}\left(\frac{a_{1}(x)}{a_{3}(x)}\right)^{\prime}-\left(2 r_{2}^{\prime}+r_{1}\right)\left(\frac{a_{1}(x)}{a_{3}(x)}\right)^{\prime}-2 r_{2}\left(\frac{a_{0}(x)}{a_{3}(x)}\right)^{\prime}-\left(3 r_{2}^{\prime \prime}+3 r_{1}^{\prime}+r_{0}\right) \frac{a_{1}(x)}{a_{3}(x)} \\
& \left.-\left(2 r_{2}^{\prime}+r_{1}\right) \frac{a_{0}(x)}{a_{3}(x)}+3 r_{0}^{\prime \prime}+r_{1}^{\prime \prime \prime}\right] \frac{d y(x)}{d x}+\left[2 r_{2} \frac{a_{0}(x)}{a_{3}(x)} \cdot\left(\frac{a_{2}(x)}{a_{3}(x)}\right)^{\prime}-r_{2}\left(\frac{a_{2}(x)}{a_{3}(x)}\right)^{\prime \prime}\right. \\
& +r_{2} \frac{a_{2}(x)}{a_{3}(x)} \cdot\left(\frac{a_{0}(x)}{a_{3}(x)}\right)^{\prime}-r_{2} \frac{a_{0}(x)}{a_{3}(x)} \cdot\left(\frac{a_{2}(x)}{a_{3}(x)}\right)^{2}+\left(3 r_{2}^{\prime}+r_{1}\right) \frac{a_{0}(x)}{a_{3}(x)} \cdot \frac{a_{2}(x)}{a_{3}(x)}+r_{0}^{\prime \prime \prime} \\
& \left.+r_{2} \frac{a_{0}(x)}{a_{3}(x)} \cdot \frac{a_{2}(x)}{a_{3}(x)}-\left(2 r_{2}^{\prime}+r_{1}\right)\left(\frac{a_{0}(x)}{a_{3}(x)}\right)^{\prime}-\left(3 r_{2}^{\prime \prime}+3 r_{1}^{\prime}+r_{0}\right) \frac{a_{0}(x)}{a_{3}(x)}\right] y(x) .
\end{aligned}
$$

We can rewrite the equation (2.2) in terms of $y(x), \frac{d y(x)}{d x}$ and $\frac{d^{2} y(x)}{d x^{2}}$ using the equations above. Then, since $\mathrm{L}_{1}$ is irreducible, all the coefficients of $y(x), \frac{d y(x)}{d x}$ and $\frac{d^{2} y(x)}{d x^{2}}$, in the equation that we will get, have to be zero. Hence, we will find a 
differential equation (2.2) for $z=r_{2} \frac{d^{2} y(x)}{d x^{2}}+r_{1} \frac{d y(x)}{d x}+r_{0} y(x)$ which has order three. We cannot present here the result because it is too large. This result is included in a Maple worksheet and is also a part of this dissertation.

\subsection{Examples}

We wrote and implemented Maple algorithms for those three transformations. All of them are in our implemented package called ODE3solve. They take as input a linear irreducible third-order differential operator and the parameters of transformation(s). The output is another linear irreducible third-order differential operator coming from the input through the transformation associated to our input transformation parameter(s).

a- The change of variables transformation:

Let us take the irreducible third-order linear differential operator $\mathrm{L}_{02}$ associated to the hypergeometric function ${ }_{0} F_{2}\left(\begin{array}{c|c}- & x \\ 1 / 7,1 / 12\end{array} \mid\right.$ and the change of variable parameter $f=\frac{2(x-7)^{2}(x-12)}{(x-3)^{2}}$. We have:

$$
\begin{gathered}
>\quad \mathrm{L} 02:=\mathrm{x}^{\wedge} 2 * \mathrm{Dx} \wedge 3+(103 / 84) * \mathrm{x} * \mathrm{Dx} \wedge 2+(1 / 84) * \mathrm{Dx}-1 ; \\
L 02:=x^{2} D x^{3}+\frac{103}{84} x D x^{2}+\frac{1}{84} D x-1 \\
>\mathrm{f}:=\operatorname{normal}((2 *(\mathrm{x}-7) \sim 2 *(\mathrm{x}-12)) /(\mathrm{x}-3) \wedge 2, \text { expanded }) ; \\
f:=\frac{2 x^{3}-52 x^{2}+434 x-1176}{x^{2}-6 x+9}
\end{gathered}
$$

To transform $\mathrm{L}_{02}$ by the change of variables with parameter $f$, we use the algorithm ChangeOfVariables in our implemented package ODE3solve

$>\quad \mathrm{L}:=$ ChangeOfVariables $(\mathrm{L} 02, \mathrm{f})$;

$$
\begin{gathered}
L:=84\left(x^{2}-2 x-75\right)^{2}(x-7)^{3}(x-12)^{3}(x-3)^{5} D x^{3}+\left(x^{2}-2 x-75\right)\left(103 x^{4}\right. \\
\left.-412 x^{3}-59390 x^{2}+913908 x-3630033\right)(x-7)^{2}(x-12)^{2}(x-3)^{4} D x^{2} \\
+(x-7)(x-12)\left(x^{8}-8 x^{7}+25948 x^{6}-1451784 x^{5}+34550774 x^{4}\right. \\
\left.-458049624 x^{3}+3589905852 x^{2}-15576038424 x+28661695857\right)(x-3)^{3} D x \\
-168(x-7)^{2}(x-12)\left(x^{2}-2 x-75\right)^{5}
\end{gathered}
$$

b- The exp-product transformation:

Let us take the irreducible third-order linear differential operator $\mathrm{L}_{12}$ associated to the hypergeometric function ${ }_{1} F_{2}\left(\begin{array}{c}1 / 3 \\ 1 / 5,1 / 9\end{array} \mid x\right)$ and the exp-product transformation parameter $r=(x-5)(x-9)$. We have:

$>\mathrm{L} 12:=\mathrm{x}^{\wedge} 2 * \mathrm{Dx} \wedge 3+(59 / 45) * \mathrm{x} * \mathrm{Dx}^{\wedge} 2+(1 / 45-\mathrm{x}) * \mathrm{Dx}-1 / 3$; 


$$
\begin{aligned}
L 12:=x^{2} D x^{3}+\frac{59}{45} x D x^{2}+(1 / 45-x) D x-1 / 3 \\
>\quad \mathrm{r}:=(\mathrm{x}-5) *(\mathrm{x}-9) ; \quad \\
r:=(x-5)(x-9)
\end{aligned}
$$

To transform $\mathrm{L}_{12}$ by the exp-product transformation with parameter $r$, we use the algorithm ExpProduct in our implemented package ODE3solve

$>\mathrm{L}:=\operatorname{ExpProduct}(\mathrm{L} 12, \mathrm{r})$;

$$
\begin{aligned}
L:= & 45 x^{2} D x^{3}-x\left(-59+135 x^{3}-1890 x^{2}+6075 x\right) D x^{2}+\left(1-5355 x-170488 x^{3}\right. \\
& \left.+276917 x^{2}+135 x^{6}-3780 x^{5}+38610 x^{4}\right) D x-1471397 x^{4}+3882779 x^{3} \\
& -4260854 x^{2}+122340 x-60-45 x^{8}+1890 x^{7}-32535 x^{6}+293909 x^{5}
\end{aligned}
$$

c- The gauge transformation:

Let us take the irreducible third-order linear differential operator $\mathrm{L}_{22}$ associated to the hypergeometric function ${ }_{2} F_{2}\left(\begin{array}{c}1 / 7,1 / 3 \\ 1 / 2,1 / 4\end{array} \mid x\right)$ and the gauge transformation parameters $r_{0}=x+1, r_{1}=0$ and $r_{2}=x^{3}$. We have:

$$
\begin{aligned}
& >\quad \mathrm{L} 22:=\mathrm{x}^{\wedge} 2 * \mathrm{Dx}^{\wedge} 3+\left(-\mathrm{x}^{\wedge} 2+(7 / 4) * \mathrm{x}\right) * \mathrm{Dx}^{\wedge} 2+(-(31 / 21) * \mathrm{x}+1 / 8) * \mathrm{Dx}-1 / 21 \text {; } \\
& L 22:=x^{2} D x^{3}+\left(-x^{2}+7 / 4 x\right) D x^{2}+\left(-\frac{31}{21} x+1 / 8\right) D x-1 / 21 \\
& >\mathrm{r} 0:=\mathrm{x}+1 \text {; } \\
& r 0:=x+1 \\
& >r 1:=0 \text {; } \\
& >\mathrm{r} 2:=\mathrm{x}^{\wedge} 3 \\
& r 1:=0 \\
& r 2:=x^{3}
\end{aligned}
$$

To transform $\mathrm{L}_{22}$ by the gauge transformation with parameters $r_{0}, r_{1}$ and $r_{2}$, we use the algorithm GaugeTransf in our implemented package ODE3solve

$$
\begin{aligned}
> & \mathrm{L}:=\text { GaugeTransf }(\mathrm{L} 22, \mathrm{r} 0, \mathrm{r} 1, \mathrm{r} 2) ; \\
L:= & 168 x^{2}\left(47424 x^{5}+97456 x^{4}+180789 x^{2}+28224+128919 x^{3}+86436 x\right) D x^{3} \\
& -42 x\left(189696 x^{6}+1006336 x^{5}+1392780 x^{4}+1367751 x^{3}+526533 x^{2}\right. \\
& -146412 x-197568) D x^{2}+\left(4173312 x^{6}+48970048 x^{5}+28542024 x^{4}\right. \\
& \left.-73928001 x^{3}-78087429 x^{2}-19261116 x+592704\right) D x-4552704 x^{5} \\
& +56356881 x^{2}+18202716 x-818496-44657216 x^{4}+16950528 x^{3}
\end{aligned}
$$

\subsection{Transformations as relations}

Solving an equation in terms of a function is like to find the relation (connection) between this equation and the equation that the considered function is solution. Hence, a transformation between differential operators can be considered as relation between them.

Remark 2.4. We can consider $\longrightarrow_{C}, \longrightarrow_{E}$ and $\longrightarrow_{G}$ as binary relations on $\mathbb{C}(x)[\partial]$. Hence, $\longrightarrow_{E}$ and $\longrightarrow_{G}$ are equivalence relations, but $\longrightarrow_{C}$ is not: the symmetry of $\longrightarrow_{C}$ 
would require algebraic functions as parameter. For example, to cancel the operation $x \mapsto x^{3}$, we would need $x \mapsto x^{1 / 3}$.

An important question when searching for transformations between two operators $\mathrm{L}_{1}$ and $\mathrm{L}_{2}$ is whether we can restrict our search to a specific order of transformations $\longrightarrow_{C}, \longrightarrow_{E}$ and $\longrightarrow_{G}$.

Lemma 2.5. Let $\mathrm{L}_{1}, \mathrm{~L}_{2}, \mathrm{~L}_{3} \in \mathrm{K}[\partial]$ be three irreducible third-order linear differential operators such that $\mathrm{L}_{1} \longrightarrow_{G} \mathrm{~L}_{2} \longrightarrow_{E} \mathrm{~L}_{3}$. Then there exists a differential operator $\mathrm{M} \in \mathrm{K}[\partial]$ such that $\mathrm{L}_{1} \longrightarrow_{E} \mathrm{M} \longrightarrow_{G} \mathrm{~L}_{3}$.

Similarly, if $\mathrm{L}_{1} \longrightarrow_{E} \mathrm{~L}_{2} \longrightarrow_{G} \mathrm{~L}_{3}$ we find $\mathrm{M} \in \mathrm{K}[\partial]$ such that $\mathrm{L}_{1} \longrightarrow_{G} \mathrm{M} \longrightarrow_{E} \mathrm{~L}_{3}$.

Proof. We use a similar proof as in [11, Lemma 2.7].

We write $\longrightarrow E G$ for any sequence of those transformations. Since they are equivalence relations, $\longrightarrow_{E G}$ is also.

Definition 2.6. We say $\mathrm{L}_{1} \in \mathrm{K}[\partial]$ is

(a) gauge equivalent to $\mathrm{L}_{2}$ if and only if $\mathrm{L}_{1} \longrightarrow_{G} \mathrm{~L}_{2}$,

(b) exp-product equivalent to $\mathrm{L}_{2}$ if and only if $\mathrm{L}_{1} \longrightarrow_{E} \mathrm{~L}_{2}$,

(c) projectively equivalent to $\mathrm{L}_{2}$ if and only if $\mathrm{L}_{1} \longrightarrow_{E G} \mathrm{~L}_{2}$.

Lemma 2.7. Let $\mathrm{L}_{1}, \mathrm{~L}_{2}, \mathrm{~L}_{3} \in \mathrm{K}[\partial]$ be three irreducible third-order linear differential operators. The following holds:

(i) $\mathrm{L}_{1} \longrightarrow_{E} \mathrm{~L}_{2} \longrightarrow_{C} \mathrm{~L}_{3} \Longrightarrow \exists \mathrm{M} \in \mathrm{K}[\partial]: \mathrm{L}_{1} \longrightarrow_{C} \mathrm{M} \longrightarrow_{E} \mathrm{~L}_{3}$,

(ii) $\mathrm{L}_{1} \longrightarrow_{G} \mathrm{~L}_{2} \longrightarrow_{C} \mathrm{~L}_{3} \Longrightarrow \exists \mathrm{M} \in \mathrm{K}[\partial]: \mathrm{L}_{1} \longrightarrow_{C} \mathrm{M} \longrightarrow_{G} \mathrm{~L}_{3}$.

Note that the converse of $(i)$ and $(i i)$ is not generally true since $\longrightarrow_{C}$ is not symmetric.

By those two lemmas above, we can then have the following statement:

Lemma 2.8. Let $\mathrm{L}_{1}, \mathrm{~L}_{2} \in \mathrm{K}[\partial]$ be two irreducible third-order linear differential operators such that $\mathrm{L}_{1} \longrightarrow \mathrm{L}_{2}$. Then there exists an operator $\mathrm{M} \in \mathrm{K}[\partial]$ such that $\mathrm{L}_{1} \longrightarrow{ }_{C} \mathrm{M} \longrightarrow_{E G}$ $\mathrm{L}_{2}$.

Proof. We just use Lemma 2.5 and Lemma 2.7 and the rest follows immediately.

\subsection{Relation between Transformations and Generalized Exponents}

Let $k$ be an extension field of $\mathbb{Q}$ which is algebraically closed and has characteristic zero (that is to be sure that the universal extension of $k(x)$ exists). Let $\mathrm{K}=k(x)$ be the field of rational functions in $x$. All of our differential operators are taken from $\mathrm{K}[\partial]$.

As it was previously said, our three known transformations connect the solutions spaces of two irreducible third-order linear differential operators. Since the solutions are related by the generalized exponents, we can think also about the way that the generalized exponents change from one solution space to another by those transformations. 


\subsubsection{Relation between Exp-product Transformation and Gener- alized Exponents}

The following lemma states how the generalized exponent varies after an exp-product transformation:

Lemma 2.9. Let $\mathrm{L}, \mathrm{M} \in \mathrm{K}[\partial]$ be two irreducible third-order linear differential operators such that $\mathrm{M} \stackrel{r}{\longrightarrow}$ E $\mathrm{L}$ and let $e$ be a generalized exponent of $\mathrm{M}$ at the point $p \in k \cup\{\infty\}$ with the ramification index $n_{e} \in \mathbb{N}^{*}$. Furthermore, let $r$ has at $p$ the series representation

$$
r=\sum_{i=m_{p}}^{+\infty} r_{i} t_{p}^{i}, \quad m_{p} \in \mathbb{Z} \quad \text { with } r_{i} \in k \quad \text { and } r_{m_{p}} \neq 0
$$

1- If $p$ is not a pole of $r$ then $m_{p} \geq 0$ and the generalized exponent of $\mathrm{L}$ at $p$ is

$$
\bar{e}= \begin{cases}e & \text { if } p \neq \infty \\ e-r_{0} t_{\infty}^{-1}-r_{1} \text { otherwise. }\end{cases}
$$

2- If $p$ is a pole of $r$ then we will have $m_{p} \leq-1$, where $-m_{p}$ is the multiplicity order of $r$ at $p$, and the generalized exponent of $\mathrm{L}$ at $p$ will be given by

$$
\bar{e}=\left\{\begin{array}{l}
e+\sum_{i=m_{p}}^{-1} r_{i} t_{p}^{i+1} \text { if } p \neq \infty \\
e-\sum_{i=m_{\infty}}^{1} r_{i} t_{\infty}^{i-1} \text { otherwise. }
\end{array}\right.
$$

Proof. Since $e$ is a generalized exponent of $\mathrm{M}$ at $p, \mathrm{M}$ has a solution of the form

$$
y=\exp \left(\int \frac{e}{t_{p}} d t_{p}\right) S
$$

for some Puiseux series $S \in k\left(\left(t_{p}^{1 / n_{e}}\right)\right)\left[\ln \left(t_{p}\right)\right]$ with non-zero constant term. The expproduct transformation, with parameter $r \in k(x)$, converts this solution into

$$
z=\exp \left(\int r d x\right) \times \exp \left(\int \frac{e}{t_{p}} d t_{p}\right) S .
$$

In order to determine the generalized exponent $\bar{e}$ of $\mathrm{L}$ at $p$ we have to rewrite (2.5) in the form:

$$
z=\exp \left(\int \frac{\bar{e}}{t_{p}} d t_{p}\right) \bar{S}
$$

for some Puiseux series $\bar{S} \in k\left(\left(t_{p}^{1 / n}\right)\right)\left[\ln \left(t_{p}\right)\right]$ with non-zero constant term and $n \in \mathbb{N}^{*}$.

1- Since $p$ is not a pole of $r$ and $r_{m_{p}} \neq 0$, we have $m_{p} \geq 0$.

$* p \neq \infty$ :

By using the fact that $t_{p}=x-p$, which means $d t_{p}=d x$, we have

$$
\exp \left(\int r d x\right)=\exp \left(\sum_{i=m_{p}}^{\infty} \frac{r_{i}}{i+1} t_{p}^{i+1}\right) \text {. }
$$


With $\exp (x)=\sum_{j=0}^{+\infty} \frac{x^{j}}{j !}$ when $x$ tends to zero, we can rewrite (2.6) as power series in $t_{p}$ since $\sum_{i=m_{p}}^{+\infty} \frac{r_{i}}{i+1} t_{p}^{i+1}$ also tends to zero when $t_{p}$ tends to zero.

$$
\begin{aligned}
\exp \left(\int r d x\right) & =\sum_{j=0}^{+\infty}\left[\frac{1}{j !} \cdot\left(\sum_{i=m_{p}}^{+\infty} \frac{r_{i}}{i+1} t_{p}^{i+1}\right)^{j}\right] \\
& =\sum_{j=0}^{+\infty} a_{j} t_{p}^{j} \quad \text { with } a_{j} \in k \text { and } a_{0}=1 .
\end{aligned}
$$

Substituting (2.7) in (2.5) we get

$$
z=\exp \left(\int \frac{e}{t_{p}} d t_{p}\right) \bar{S}
$$

where $\bar{S}=S \cdot \sum_{j=0}^{+\infty} a_{j} t_{p}^{j} \in k\left(\left(t_{p}^{1 / n_{e}}\right)\right)\left[\ln \left(t_{p}\right)\right]$ has a non-zero constant term.

* $\underline{p=\infty}:$

Since $t_{\infty}=1 / x$ we have $d t_{\infty}=-\frac{1}{x^{2}} d x=-t_{\infty}^{2} d x$ which means $d x=-t_{\infty}^{-2} d t_{\infty}$. Hence,

$$
\begin{aligned}
\exp \left(\int r d x\right) & =\exp \left(\int\left(r_{0}+r_{1} t_{\infty}\right) d x\right) \exp \left(\int\left(\sum_{i=2}^{+\infty} r_{i} t_{\infty}^{i}\right) d x\right) \\
& =\exp \left(\int\left(r_{0}+r_{1} t_{\infty}\right)\left(-t_{\infty}^{-2}\right) d t_{\infty}\right) \exp \left(\int\left(\sum_{i=2}^{+\infty} r_{i} t_{\infty}^{i}\right)\right. \\
& \left.\times\left(-t_{\infty}^{-2}\right) d t_{\infty}\right) \\
& =\exp \left(-\int \frac{r_{0} t_{\infty}^{-1}+r_{1}}{t_{\infty}} d t_{\infty}\right) \exp \left(\sum_{i=2}^{+\infty} \frac{r_{i}}{i-1} t_{\infty}^{i-1}\right)
\end{aligned}
$$

With $\exp (x)=\sum_{j=0}^{+\infty} \frac{x^{j}}{j !}$ when $x$ tends to zero, we can rewrite the second right factor in (2.8) as power series in $t_{\infty}$ since $\sum_{i=2}^{+\infty} \frac{r_{i}}{i-1} t_{\infty}^{i-1}$ also tends to zero when $t_{\infty}$ tends to zero.

$$
\begin{aligned}
\exp \left(\sum_{i=2}^{+\infty} \frac{r_{i}}{i-1} t_{\infty}^{i-1}\right) & =\sum_{j=0}^{+\infty}\left[\frac{1}{j !} \cdot\left(\sum_{i=2}^{+\infty} \frac{r_{i}}{i-1} t_{\infty}^{i-1}\right)^{j}\right] \\
& =\sum_{j=0}^{+\infty} a_{j} t_{\infty}^{j} \quad \text { with } a_{j} \in k \text { and } a_{0}=1
\end{aligned}
$$

Substituting (2.9) in (2.8) and then the result in (2.5) we get

$$
z=\exp \left(\int \frac{e-r_{0} t_{\infty}^{-1}-r_{1}}{t_{\infty}} d t_{\infty}\right) \bar{S}
$$


where $\bar{S}=S \cdot \sum_{j=0}^{+\infty} a_{j} t_{\infty}^{j} \in k\left(\left(t_{\infty}^{1 / n_{e}}\right)\right)\left[\ln \left(t_{\infty}\right)\right]$ has a non-zero constant term.

2- Since $p$ is a pole of $r$ and $r_{m_{p}} \neq 0$ then $m_{p} \leq-1$, and $-m_{p}$ is the multiplicity order of $r$ at $p$.

$* p \neq \infty$ :

We have to handle the positive and negative powers of $t_{p}$ in $r$ separately. Here (2.5) becomes

$$
z=\exp \left(\int\left(\sum_{i=m_{p}}^{-1} r_{i} t_{p}^{i}\right) d x\right) \cdot \exp \left(\int\left(\sum_{i=0}^{+\infty} r_{i} t_{p}^{i}\right) d x\right) \cdot \exp \left(\int \frac{e}{t_{p}} d t_{p}\right) S .
$$

By using the fact that $t_{p}=x-p$, which means $d t_{p}=d x$, the positive powers of $t_{p}$ in $r$ become

$$
\exp \left(\int\left(\sum_{i=0}^{+\infty} r_{i} t_{p}^{i}\right) d x\right)=\exp \left(\sum_{i=0}^{+\infty} \frac{r_{i}}{i+1} t_{p}^{i+1}\right)
$$

With $\exp (x)=\sum_{j=0}^{+\infty} \frac{x^{j}}{j !}$ when $x$ tends to zero, we can rewrite (2.11) as power series in $t_{p}$ since $\sum_{i=0}^{+\infty} \frac{r_{i}}{i+1} t_{p}^{i+1}$ also tends to zero when $t_{p}$ tends to zero.

$$
\begin{aligned}
\exp \left(\int\left(\sum_{i=0}^{+\infty} r_{i} t_{p}^{i}\right) d x\right) & =\sum_{j=0}^{+\infty}\left[\frac{1}{j !} \cdot\left(\sum_{i=0}^{+\infty} \frac{r_{i}}{i+1} t_{p}^{i+1}\right)^{j}\right] \\
& =\sum_{j=0}^{+\infty} a_{j} t_{p}^{j} \quad \text { with } a_{j} \in k \text { and } a_{0}=1 .
\end{aligned}
$$

The negative powers of $t_{p}$ in the series expansion of $r$ becomes a part of the generalized exponent:

$$
\exp \left(\int\left(\sum_{i=m_{p}}^{-1} r_{i} t_{p}^{i}\right) d x\right)=\exp \left(\int\left[\frac{1}{d t_{p}} \cdot \sum_{i=m_{p}}^{-1} r_{i} t_{p}^{i+1}\right] d t_{p}\right) .
$$

Substituting (2.12) and (2.13) in (2.10) we get

$$
z=\exp \left(\int\left[\frac{1}{d t_{p}} \cdot\left(e+\sum_{i=m_{p}}^{-1} r_{i} t_{p}^{i+1}\right)\right] d t_{p}\right) \bar{S}
$$

where $\bar{S}=S \cdot \sum_{j=0}^{+\infty} a_{j} t_{p}^{j} \in k\left(\left(t_{p}^{1 / n_{e}}\right)\right)\left[\ln \left(t_{p}\right)\right]$ has a non-zero constant term.

* $p=\infty$ :

Since $t_{\infty}=1 / x$, we have $d t_{\infty}=-\frac{1}{x^{2}} d x=-t_{\infty}^{2} d x$ which means $d x=-t_{\infty}^{-2} d t_{\infty}$. 
Hence,

$$
\begin{aligned}
\exp \left(\int r d x\right) & =\exp \left(\int\left(\sum_{i=m_{\infty}}^{1} r_{i} t_{\infty}^{i}\right) d x\right) \cdot \exp \left(\int\left(\sum_{i=2}^{+\infty} r_{i} t_{\infty}^{i}\right) d x\right) \\
& =\exp \left(\int\left(\sum_{i=m_{\infty}}^{1} r_{i} t_{\infty}^{i}\right)\left(-t_{\infty}^{-2}\right) d t_{\infty}\right) \cdot \exp \left(\int\left(\sum_{i=2}^{+\infty} r_{i} t_{\infty}^{i}\right)\right. \\
& =\exp \left(-\int \frac{\left.\left.t_{\infty}^{-2}\right) d t_{\infty}\right)}{t_{\infty}} d t_{\infty}\right) \cdot \exp \left(\sum_{i=2}^{+\infty} \frac{r_{i}}{i-1} t_{\infty}^{i-1}\right) \cdot(2.1
\end{aligned}
$$

With $\exp (x)=\sum_{j=0}^{+\infty} \frac{x^{j}}{j !}$ when $x$ tends to zero, we can rewrite the second right factor in (2.8) as power series in $t_{\infty}$ since $\sum_{i=2}^{+\infty} \frac{r_{i}}{i-1} t_{\infty}^{i-1}$ also tends to zero when $t_{\infty}$ tends to zero.

$$
\begin{aligned}
\exp \left(\sum_{i=2}^{+\infty} \frac{r_{i}}{i-1} t_{\infty}^{i-1}\right) & =\sum_{j=0}^{+\infty}\left[\frac{1}{j !} \cdot\left(\sum_{i=2}^{+\infty} \frac{r_{i}}{i-1} t_{\infty}^{i-1}\right)^{j}\right] \\
& =\sum_{j=0}^{+\infty} a_{j} t_{\infty}^{j} \quad \text { with } a_{j} \in k \text { and } a_{0}=1
\end{aligned}
$$

Substituting (2.15) in (2.14) and then the result in (2.5) we get

$$
z=\exp \left(\int \frac{e-\sum_{i=m_{\infty}}^{1} r_{i} t_{\infty}^{i-1}}{t_{\infty}} d t_{\infty}\right) \bar{S}
$$

where $\bar{S}=S \cdot \sum_{j=0}^{+\infty} a_{j} t_{\infty}^{j} \in k\left(\left(t_{\infty}^{1 / n_{e}}\right)\right)\left[\ln \left(t_{\infty}\right)\right]$ has a non-zero constant term.

\section{Example}

Let us take the irreducible third-order linear differential operator $\mathrm{L}_{02}$ associated to the hypergeometric function ${ }_{0} F_{2}\left(\begin{array}{c|c}- \\ 1 / 7,1 / 12\end{array} \mid x\right)$ and the exp-product parameter $r=\frac{2(x-7)^{2}(x-12)}{(x-3)^{5}}$ such that $\mathrm{L}_{02} \stackrel{r}{\longrightarrow}_{E} \mathrm{M}$.

$>\quad \mathrm{L} 02:=\mathrm{x}^{\wedge} 2 * \mathrm{Dx}^{\wedge} 3+(103 / 84) * \mathrm{x} * \mathrm{Dx}^{\wedge} 2+(1 / 84) * \mathrm{Dx}-1$; 
$>\quad M:=\operatorname{ExpProduct}(\mathrm{L} 02, \mathrm{r})$;

$$
L 02:=x^{2} D x^{3}+\frac{103}{84} x D x^{2}+\frac{1}{84} D x-1
$$

$$
\begin{aligned}
M:= & 84 x^{2}(x-3)^{20} D x^{3}+x\left(103 x^{5}-2049 x^{4}+22374 x^{3}-137178 x^{2}+338067 x\right. \\
& -25029)(x-3)^{15} D x^{2}+\left(x^{10}+566 x^{9}-28567 x^{8}+487772 x^{7}-3652074 x^{6}\right. \\
& +9698904 x^{5}+24950142 x^{4}-208055196 x^{3}+375197913 x^{2}-59065038 x \\
& +59049)(x-3)^{10} D x-2\left(42 x^{15}-1890 x^{14}+39989 x^{13}-536323 x^{12}\right. \\
& +5165621 x^{11}-37389819 x^{10}+198814604 x^{9}-703239656 x^{8}+1187929356 x^{7} \\
& +1661566320 x^{6}-12458079627 x^{5}+17843929317 x^{4}+15530253525 x^{3} \\
& \left.-53256919635 x^{2}+15808471434 x-637374906\right)(x-3)^{5}
\end{aligned}
$$

- $x=0$ is not a pole of $r$ and the generalized exponents of $\mathrm{L}_{02}$ at $x=0$ are

$>$ gen_exp $(\mathrm{L} 02, \mathrm{t}, \mathrm{x}=0)$;

$$
\left[[0, t=x],\left[\frac{11}{12}, t=x\right],[6 / 7, t=x]\right]
$$

For the operator $\mathrm{M}$ we have

$>\quad$ gen_exp $(M, t, x=0)$;

$$
\left[[0, t=x],\left[\frac{11}{12}, t=x\right],[6 / 7, t=x]\right]
$$

So the generalized exponents of $\mathrm{L}_{02}$ and $\mathrm{M}$ at $x=0$ are the same (that can be seen by the part 1- of Lemma 2.9).

- $x=\infty$ is not a pole of $r$ and the generalized exponents of $\mathrm{L}_{02}$ at $x=\infty$ are

$>\quad$ gen_exp(L02,t,x=infinity);

$$
\left[\left[t^{-1}-\frac{65}{252},-t^{3}=x^{-1}\right]\right]
$$

Let $t_{1}, t_{2}$ and $t_{3}$ be the roots of the equation $-t^{3}=1 / x$ where $t$ is the unknown. Let $e_{1}=t_{1}^{-1}-\frac{65}{252}, e_{2}=t_{2}^{-1}-\frac{65}{252}$ and $e_{3}=t_{3}{ }^{-1}-\frac{65}{252}$.

For the operator $M$ we have

$>\quad \operatorname{gen} \exp (\mathrm{M}, \mathrm{t}, \mathrm{x}=$ infinity $)$;

$$
\left[\left[t^{-1}-\frac{65}{252},-t^{3}=x^{-1}\right]\right]
$$

So $\bar{e}_{1}=t_{1}{ }^{-1}-\frac{65}{252}, \bar{e}_{2}=t_{2}{ }^{-1}-\frac{65}{252}$ and $\bar{e}_{3}=t_{3}{ }^{-1}-\frac{65}{252}$ will be the generalized exponents of M at $x=\infty$.

The series expansion of $r$ at $x=\infty$, with coefficient $r_{i}$, is

$>\operatorname{series}(r, x=$ infinity);

$$
2 x^{-2}-22 x^{-3}-76 x^{-4}+204 x^{-5}+O\left(x^{-6}\right)
$$

So $r=2 t_{\infty}{ }^{2}-22 t_{\infty}{ }^{3}-76 t_{\infty}{ }^{4}+204 t_{\infty}{ }^{5}+O\left(t_{\infty}{ }^{6}\right)$ and hence $r_{0}=0$ and $r_{1}=0$. Therefore, for $j=1,2,3$, we have

$$
e_{j}-r_{0} t_{\infty}^{-1}-r_{1}=e_{j}-0 \times t_{\infty}^{-1}-0=e_{j}=\bar{e}_{j} .
$$

So the part 1- of our Lemma 2.9 is satisfied. 
- $x=3$ is a pole of $r$ of order 5 and the generalized exponents of $\mathrm{L}_{02}$ at $x=3$ are

$>\quad \operatorname{gen} \_\exp (\mathrm{L} 02, \mathrm{t}, \mathrm{x}=3)$;

$$
[[0,1,2, t=x-3]]
$$

Let $e_{1}=0, e_{2}=1$ and $e_{3}=2$.

For the operator $\mathrm{M}$ we have

$$
\begin{aligned}
& >\quad \operatorname{gen} \_\exp (\mathrm{M}, \mathrm{t}, \mathrm{x}=3) ; \\
& {\left[\left[2 t^{-1}-34 t^{-2}+176 t^{-3}-288 t^{-4}, 1+2 t^{-1}-34 t^{-2}+176 t^{-3}-288 t^{-4},\right.\right.} \\
& \left.\left.2+2 t^{-1}-34 t^{-2}+176 t^{-3}-288 t^{-4}, t=x-3\right]\right]
\end{aligned}
$$

Let $\bar{e}_{1}=2 t^{-1}-34 t^{-2}+176 t^{-3}-288 t^{-4}, \bar{e}_{2}=1+2 t^{-1}-34 t^{-2}+176 t^{-3}-288 t^{-4}$ and $\bar{e}_{3}=2+2 t^{-1}-34 t^{-2}+176 t^{-3}-288 t^{-4}$. The series representation of $r$ at $x=3$, with coefficient $r_{i}$, is

$>\operatorname{series}(r, \mathrm{x}=3)$;

$$
-288(x-3)^{-5}+176(x-3)^{-4}-34(x-3)^{-3}+2(x-3)^{-2}
$$

Hence $\sum_{i=-5}^{-1} r_{i} t_{3}^{i+1}=-288(x-3)^{-4}+176(x-3)^{-3}-34(x-3)^{-2}+2(x-3)^{-1}$.

$$
\left\{\begin{aligned}
e_{1}+\sum_{i=-5}^{-1} r_{i} t_{3}^{i+1} & =0-288(x-3)^{-4}+176(x-3)^{-3}-34(x-3)^{-2}+2(x-3)^{-1} \\
& =\bar{e}_{1}, \\
e_{2}+\sum_{i=-5}^{-1} r_{i} t_{3}^{i+1} & =1-288(x-3)^{-4}+176(x-3)^{-3}-34(x-3)^{-2}+2(x-3)^{-1} \\
& =\bar{e}_{2}, \\
e_{3}+\sum_{i=-5}^{-1} r_{i} t_{3}^{i+1} & =2-288(x-3)^{-4}+176(x-3)^{-3}-34(x-3)^{-2}+2(x-3)^{-1} \\
& =\bar{e}_{3} .
\end{aligned}\right.
$$

So the part 2- of our Lemma 2.9 is satisfied.

Let us now take the exp-product parameter $r=(x-5)(x-9)$ such that $\mathrm{L}_{02} \stackrel{r}{\longrightarrow}{ }_{E} \mathrm{M}$.

$$
\begin{aligned}
>\mathrm{r}: & =(\mathrm{x}-5) *(\mathrm{x}-9) ; \\
>\quad \mathrm{M}: & =\text { ExpProduct }(\mathrm{L} 02, \mathrm{r}) ; \\
M:= & 84 x^{2} D x^{3}-x\left(-103+252 x^{3}-3528 x^{2}+11340 x\right) D x^{2}+\left(252 x^{6}-318230 x^{3}\right. \\
& \left.+1+516712 x^{2}-9270 x-7056 x^{5}+72072 x^{4}\right) D x-2746408 x^{4}+7245730 x^{3} \\
& -7943415 x^{2}+210031 x-129-84 x^{8}+3528 x^{7}-60732 x^{6}+548623 x^{5}
\end{aligned}
$$

$x=\infty$ is a pole of $r$ of order 2. By the computations above, the generalized exponents of $\mathrm{L}_{02}$ at $x=\infty$ are $e_{1}=t_{1}{ }^{-1}-\frac{65}{252}, e_{2}=t_{2}{ }^{-1}-\frac{65}{252}$ and $e_{3}=t_{3}{ }^{-1}-\frac{65}{252}$ where $t_{1}, t_{2}$ and $t_{3}$ are the roots of the equation $-t^{3}=1 / x$ with the unknown $t$.

For the operator $\mathrm{M}$ we have

$>\quad \operatorname{gen} \_\exp (\mathrm{M}, \mathrm{t}, \mathrm{x}=$ infinity);

$$
\left[\left[t^{-1}-\frac{65}{252}+45 t^{-3}+14 t^{-6}+t^{-9},-t^{3}=x^{-1}\right]\right]
$$


So $\bar{e}_{1}=1 / t_{1}-65 / 252+45 / t_{1}{ }^{3}+14 / t_{1}{ }^{6}+1 / t_{1}{ }^{9}, \bar{e}_{2}=1 / t_{2}-65 / 252+45 / t_{2}{ }^{3}+$ $14 / t_{2}{ }^{6}+1 / t_{2}{ }^{9}$ and $\bar{e}_{3}=1 / t_{3}-65 / 252+45 / t_{3}{ }^{3}+14 / t_{3}{ }^{6}+1 / t_{3}{ }^{9}$ will be the generalized exponents of $\mathrm{M}$ at $x=\infty$.

The series expansion of $r$ at $x=\infty$, with coefficient $r_{i}$, is

$>\operatorname{series}(r, x=i n f i n i t y)$;

$$
x^{2}-14 x+45
$$

So $r=t_{\infty}^{-2}-14 t_{\infty}^{-1}+45$ and hence

$$
\begin{aligned}
\sum_{i=-2}^{1} r_{i} t_{\infty}^{i-1} & =t_{\infty}^{-3}-14 t_{\infty}^{-2}+45 t_{\infty}^{-1} \\
& =\left(-t^{3}\right)^{-3}-14\left(-t^{3}\right)^{-2}+45\left(-t^{3}\right)^{-1} \text { for }-t^{3}=1 / x=t_{\infty} \\
& =-t^{-9}-14 t^{-6}-45 t^{-3} \text { for }-t^{3}=1 / x=t_{\infty}
\end{aligned}
$$

Therefore, for $j=1,2,3$, we have

$$
\begin{aligned}
e_{j}-\sum_{i=-2}^{1} r_{i} t_{\infty}^{i-1} & =t_{j}^{-1}-\frac{65}{252}-\left(-t^{-9}-14 t^{-6}-45 t^{-3}\right) \quad \text { for } \quad-t^{3}=1 / x=t_{\infty} \\
& =t_{j}{ }^{-1}-\frac{65}{252}-\left(-t_{j}{ }^{-9}-14 t_{j}{ }^{-6}-45 t_{j}^{-3}\right) \quad \text { since } \quad-t_{j}{ }^{3}=1 / x=t_{\infty} \\
& =t_{j}{ }^{-1}-\frac{65}{252}+t_{j}{ }^{-9}+14 t_{j}{ }^{-6}+45 t_{j}{ }^{-3} \\
& =\bar{e}_{j} .
\end{aligned}
$$

So the part 2- of our Lemma 2.9 is satisfied.

\subsubsection{Relation between Gauge Transformation and Generalized Exponents}

The following lemma states how the generalized exponent varies after a gauge transformation:

Lemma 2.10. Let $\mathrm{L}, \mathrm{M} \in \mathbb{C}(x)[\partial]$ be two irreducible third-order linear differential operators such that $\mathrm{M} \longrightarrow_{G} \mathrm{~L}$ and let $e$ be a generalized exponent of $\mathrm{M}$ at the point $p$. The operator $\mathrm{L}$ has at $p$ a generalized exponent $\bar{e}$ such that $\bar{e}=e \bmod \frac{1}{n_{e}} \mathbb{Z}$, where $n_{e} \in \mathbb{N}^{*}$ is the ramification index of $e$.

Proof. Since $e$ is a generalized exponent of $\mathrm{M}$ at $p$, then $\mathrm{M}$ has a solution of the form

$$
y=\exp \left(\int \frac{e}{t_{p}} d t_{p}\right) S
$$

for some Puiseux series $S \in k\left(\left(t_{p}^{1 / n_{e}}\right)\right)\left[\ln \left(t_{p}\right)\right]$ with non-zero constant term. The gauge transformation, with parameter $r_{0}, r_{1}, r_{2} \in k(x)$, converts this solution into

$$
z=r_{2} y^{\prime \prime}+r_{1} y^{\prime}+r_{0} y \text {. }
$$

In order to determine the generalized exponent $\bar{e}$ of $\mathrm{L}$ at $p$ we have to rewrite (2.17) in the form:

$$
z=\exp \left(\int \frac{\bar{e}}{t_{p}} d t_{p}\right) \bar{S}
$$


for some Puiseux series $\bar{S} \in k\left(\left(t_{p}^{1 / n}\right)\right)\left[\ln \left(t_{p}\right)\right]$ with non-zero constant term and $n \in \mathbb{N}^{*}$.

Let, for $i \in\{0,1,2\}, r_{i}$ has at $p$ the series representation

$$
r_{i}=\sum_{j=m_{i}}^{+\infty} a_{i, j} t_{p}^{j} \quad m_{i} \in \mathbb{Z} \quad \text { with } \quad a_{i, j} \in k \quad \text { and } \quad a_{i, m_{i}} \neq 0 .
$$

Then, for $i \in\{0,1,2\}$

$$
r_{i}=t_{p}^{m_{i}} \sum_{j=0}^{+\infty} a_{i, j+m_{i}} t_{p}^{j}
$$

Since $\sum_{j=0}^{+\infty} a_{i, j+m_{i}} t_{p}^{j} \in k\left(t_{p}\right) \subseteq k\left(\left(t_{p}^{1 / n_{e}}\right)\right)$ then

$$
r_{i}=t_{p}^{m_{i}} \bar{S}_{i}
$$

for some Puiseux series $\bar{S}_{i} \in k\left(\left(t_{p}^{1 / n_{e}}\right)\right)\left[\ln \left(t_{p}\right)\right]$ with non-zero constant term.

Since $\mathrm{M}$ is an irreducible third-order operator, then $y, y^{\prime}$ and $y^{\prime \prime}$ are linearly independent. That means in particular they cannot be zero.

$$
\begin{aligned}
y^{\prime} & =\frac{e}{t_{p}} \exp \left(\int \frac{e}{t_{p}} d t_{p}\right) S+\exp \left(\int \frac{e}{t_{p}} d t_{p}\right) S^{\prime} \\
& =\exp \left(\int \frac{e}{t_{p}} d t_{p}\right)\left[\frac{e}{t_{p}} S+S^{\prime}\right] .
\end{aligned}
$$

With the fact that $y^{\prime} \neq 0$ and $S$ is a Puiseux series, we can conclude that $\frac{e}{t_{p}} S+S^{\prime}$ is a non-null Puiseux series, and can be written as

$$
\frac{e}{t_{p}} S+S^{\prime}=t_{p}^{\alpha_{1}} \tilde{S}
$$

where $\alpha_{1} \in \frac{1}{n_{e}} \mathbb{Z}$ and $\tilde{S} \in k\left(\left(t_{p}^{1 / n_{e}}\right)\right)\left[\ln \left(t_{p}\right)\right]$ a Puiseux series with non-zero constant term. Hence

$$
\begin{gathered}
y^{\prime}=t_{p}^{\alpha_{1}} \tilde{S} \cdot \exp \left(\int \frac{e}{t_{p}} d t_{p}\right) \\
=\exp \left(\int \frac{e+\alpha_{1}}{t_{p}} d t_{p}\right) \tilde{S} . \\
y^{\prime \prime}=\frac{e+\alpha_{1}}{t_{p}} \exp \left(\int \frac{e+\alpha_{1}}{t_{p}} d t_{p}\right) \tilde{S}+\exp \left(\int \frac{e+\alpha_{1}}{t_{p}} d t_{p}\right) \tilde{S}^{\prime} \\
=\exp \left(\int \frac{e+\alpha_{1}}{t_{p}} d t_{p}\right)\left[\frac{e+\alpha_{1}}{t_{p}} \tilde{S}+\tilde{S}^{\prime}\right] .
\end{gathered}
$$

With the fact that $y^{\prime \prime} \neq 0, \alpha_{1} \in \frac{1}{n_{e}} \mathbb{Z}$ and $\tilde{S}$ is a Puiseux series, we can conclude that $\frac{e+\alpha_{1}}{t_{p}} \tilde{S}+\tilde{S}^{\prime}$ is a non-null Puiseux series, and can be written as

$$
\frac{e+\alpha_{1}}{t_{p}} \tilde{S}+\tilde{S}^{\prime}=t_{p}^{\alpha_{2}} \tilde{\tilde{S}}
$$


where $\alpha_{2} \in \frac{1}{n_{e}} \mathbb{Z}$ and $\tilde{\tilde{S}} \in k\left(\left(t_{p}^{1 / n_{e}}\right)\right)\left[\ln \left(t_{p}\right)\right]$ is a Puiseux series with non-zero constant term. Hence,

$$
\begin{aligned}
y^{\prime \prime} & =t_{p}^{\alpha_{2}} \tilde{\tilde{S}} \cdot \exp \left(\int \frac{e+\alpha_{1}}{t_{p}} d t_{p}\right) \\
& =\exp \left(\int \frac{e+\alpha_{1}+\alpha_{2}}{t_{p}} d t_{p}\right) \tilde{\tilde{S}} .
\end{aligned}
$$

By using (2.17), (2.18), (2.19) and (2.22), our equation (2.17) becomes

$$
\begin{aligned}
z= & t_{p}^{m_{0}} \bar{S}_{0} \cdot \exp \left(\int \frac{e}{t_{p}} d t_{p}\right) S+t_{p}^{m_{1}} \bar{S}_{1} \cdot \exp \left(\int \frac{e+\alpha_{1}}{t_{p}} d t_{p}\right) \tilde{S} \\
& +t_{p}^{m_{2}} \bar{S}_{2} \cdot \exp \left(\int \frac{e+\alpha_{1}+\alpha_{2}}{t_{p}} d t_{p}\right) \tilde{\tilde{S}} \\
= & \exp \left(\int \frac{e}{t_{p}} d t_{p}\right)\left[t_{p}^{m_{0}} \bar{S}_{0} S+t_{p}^{m_{1}+\alpha_{1}} \bar{S}_{1} \tilde{S}+t_{p}^{m_{2}+\alpha_{1}+\alpha_{2}} \bar{S}_{2} \tilde{\tilde{S}}\right] .
\end{aligned}
$$

With the fact that $z \neq 0,\left\{m_{0}, m_{1}, m_{2}, \alpha_{1}, \alpha_{2}\right\} \subset \frac{1}{n_{e}} \mathbb{Z}$, and $S, \bar{S}_{0}, \bar{S}_{1}, \bar{S}_{2}, \tilde{S}$ and $\tilde{\tilde{S}}$ are Puiseux series, we can conclude that $t_{p}^{m_{0}} \bar{S}_{0} S+t_{p}^{m_{1}+\alpha_{1}} \bar{S}_{1} \tilde{S}+t_{p}^{m_{2}+\alpha_{1}+\alpha_{2}} \bar{S}_{2} \tilde{\tilde{S}}$ is a non-null Puiseux series, and can be written as

$$
t_{p}^{m_{0}} \bar{S}_{0} S+t_{p}^{m_{1}+\alpha_{1}} \bar{S}_{1} \tilde{S}+t_{p}^{m_{2}+\alpha_{1}+\alpha_{2}} \bar{S}_{2} \tilde{\tilde{S}}=t_{p}^{\alpha} \bar{S}
$$

where $\alpha \in \frac{1}{n_{e}} \mathbb{Z}$ and $\bar{S} \in k\left(\left(t_{p}^{1 / n_{e}}\right)\right)\left[\ln \left(t_{p}\right)\right]$ is a Puiseux series with non-zero constant term. Hence

$$
\begin{aligned}
z & =t_{p}^{\alpha} \bar{S} \cdot \exp \left(\int \frac{e}{t_{p}} d t_{p}\right) \\
& =\exp \left(\int \frac{e+\alpha}{t_{p}} d t_{p}\right) \bar{S}
\end{aligned}
$$

\section{Example}

Let us take the irreducible third-order linear differential operator $\mathrm{L}_{12}$ associated to the hypergeometric function ${ }_{1} F_{2}\left(\begin{array}{c|c}1 / 3 \\ 1 / 7,1 / 12\end{array} \mid x\right)$ and the gauge parameters $r_{0}=\frac{3}{x}, r_{1}=\frac{x}{x-7}$ and $r_{2}=x-7$ such that $\mathrm{L}_{12} \stackrel{r_{0}, r_{1}, r_{2}}{\longrightarrow} G \mathrm{M}$.

$>\quad \mathrm{L} 12:=\mathrm{x}^{\wedge} 2 * \mathrm{Dx}^{\wedge} 3+(103 / 84) * \mathrm{x} * \mathrm{Dx}^{\wedge} 2+(1 / 84-\mathrm{x}) * \mathrm{Dx}-1 / 3$;

$>\mathrm{r} 0:=3 / \mathrm{x}$

$$
L 12:=x^{2} D x^{3}+\frac{103}{84} x D x^{2}+\left(\frac{1}{84}-x\right) D x-1 / 3
$$

$$
r 0:=3 x^{-1}
$$




$$
\begin{aligned}
> & \mathrm{r} 1:=\mathrm{x} /(\mathrm{x}-7) ; \\
r 1:=\frac{x}{x-7} & r 2:=x-1 \\
> & \mathrm{r} 2:=\mathrm{x}-1 ; \\
>\quad & \text { M }:=\text { GaugeTransf }(\mathrm{L} 12, \mathrm{r} 0, \mathrm{r} 1, \mathrm{r} 2) ; \\
M:= & 84 x^{3}(x-7)^{2}\left(21952 x^{7}-498540 x^{6}+3209213 x^{5}-6210396 x^{4}+7536812 x^{3}\right. \\
& \left.-498540 x^{6}-24756039 x^{2}+1510327 x-1029\right)^{2} D x^{3}+x^{2}(x-7)\left(21952 x^{7}\right. \\
& \left.+3209213 x^{5}-6210396 x^{4}+7536812 x^{3}-24756039 x^{2}+1510327 x-1029\right) \\
& \times\left(4105024 x^{8}-125116180 x^{7}+1205576435 x^{6}-5040206417 x^{5}+12071161040 x^{4}\right. \\
& \left.-29324248037 x^{3}+62562392872 x^{2}-4642032346 x+3767169\right) D x^{2}-x(x-7) \\
& \times\left(1843968 x^{9}-56651056 x^{8}-2782269063 x^{6}+7351831423 x^{5}-25304734510 x^{4}\right. \\
& \left.+595006692 x^{7}+52630605482 x^{3}-39362575777 x^{2}+4084231683 x-4653138\right) \\
& \times\left(21952 x^{7}-498540 x^{6}+3209213 x^{5}-6210396 x^{4}+7536812 x^{3}-24756039 x^{2}\right. \\
& +1510327 x-1029) D x-(x-7)\left(2458624 x^{9}-82127920 x^{8}+972393772 x^{7}\right. \\
& -5432120281 x^{6}+16952067129 x^{5}-35816091042 x^{4}+40872253492 x^{3} \\
& \left.-4796991661 x^{2}+142564863 x-749112\right)\left(21952 x^{7}-498540 x^{6}+3209213 x^{5}\right. \\
& \left.-6210396 x^{4}+7536812 x^{3}-24756039 x^{2}+1510327 x-1029\right)
\end{aligned}
$$

- The generalized exponents of $\mathrm{L}_{12}$ at $x=0$ are

$>\quad$ gen_exp $(\mathrm{L} 12, \mathrm{t}, \mathrm{x}=0)$;

$$
\left[[0, t=x],\left[\frac{11}{12}, t=x\right],[6 / 7, t=x]\right]
$$

Let $e_{1}=0, e_{2}=\frac{11}{12}$ and $e_{3}=\frac{6}{7}$ be those generalized exponents. Their ramification indices are $n_{e_{1}}=n_{e_{2}}=n_{e_{3}}=1$.

For the operator $\mathrm{M}$ we have

$$
\begin{aligned}
& >\quad \operatorname{gen} \_\exp (\mathrm{M}, \mathrm{t}, \mathrm{x}=0) \\
& \qquad\left[[-1, t=x],\left[-\frac{8}{7}, t=x\right],\left[-\frac{13}{12}, t=x\right]\right]
\end{aligned}
$$

So $\bar{e}_{1}=-1, \bar{e}_{2}=-\frac{13}{12}$ and $\bar{e}_{3}=-\frac{8}{7}$ will be the generalized exponents of $\mathrm{M}$ at $x=0$.

$$
\left\{\begin{array}{l}
\bar{e}_{1}-e_{1}=-1-0=-1 \in \mathbb{Z}=\frac{1}{n_{e_{1}}} \mathbb{Z} \\
\bar{e}_{2}-e_{2}=-\frac{13}{12}-\frac{11}{12}=-2 \in \mathbb{Z}=\frac{1}{n_{e_{2}}} \mathbb{Z} \\
\bar{e}_{3}-e_{3}=-\frac{8}{7}-\frac{6}{7}=-2 \in \mathbb{Z}=\frac{1}{n_{e_{3}}} \mathbb{Z}
\end{array}\right.
$$

- The generalized exponents of $\mathrm{L}_{12}$ at $x=\infty$ are

$>$ gen_exp(L12,t, $x=$ infinity); 


$$
\left[\left[1 / 3, t=x^{-1}\right],\left[t^{-1}-\frac{17}{56}, t^{2}=x^{-1}\right]\right]
$$

Let $e_{1}=\frac{1}{3}, e_{2}=t_{1}{ }^{-1}-\frac{17}{56}$ and $e_{3}=t_{2}{ }^{-1}-\frac{17}{56}$ be those generalized exponents where $t_{1}$ and $t_{2}$ are solutions of the equation $t^{2}=x^{-1}$ with unknown $t$. The ramification index of $e_{1}$ is $n_{e_{1}}=1$ but for $e_{2}$ and $e_{3}$ we have $n_{e_{2}}=n_{e_{3}}=2$.

For the operator $M$ we have

$>\quad \operatorname{gen} \_\exp (M, t, x=i n f$ inity $)$;

$$
\left[\left[4 / 3, t=x^{-1}\right],\left[t^{-1}-\frac{17}{56}, t^{2}=x^{-1}\right]\right]
$$

So $\bar{e}_{1}=\frac{4}{3}, \bar{e}_{2}=t_{1}{ }^{-1}-\frac{17}{56}$ and $\bar{e}_{3}=t_{2}{ }^{-1}-\frac{17}{56}$ will be the generalized exponents of $\mathrm{M}$ at $x=\infty$ where $t_{1}$ and $t_{2}$ are solutions of the equation $t^{2}=x^{-1}$ with unknown $t$.

$$
\left\{\begin{array}{l}
\frac{4}{3}-\frac{1}{3}=1 \in \mathbb{Z}=\frac{1}{\bar{e}_{1}-e_{1}=\mathbb{Z},} \\
\bar{e}_{2}-e_{2}=t_{1}^{-1}-\frac{17}{56}-\left(t_{1}^{-1}-\frac{17}{56}\right)=0 \in \frac{1}{n_{e_{2}}} \mathbb{Z} \\
\bar{e}_{3}-e_{3}=t_{2}^{-1}-\frac{17}{56}-\left(t_{2}^{-1}-\frac{17}{56}\right)=0 \in \frac{1}{n_{e_{3}}} \mathbb{Z} .
\end{array}\right.
$$

- The generalized exponents of $\mathrm{L}_{12}$ at $x=7$ are

$>$ gen_exp $(\mathrm{L} 12, \mathrm{t}, \mathrm{x}=7)$;

$$
[[0,1,2, t=x-7]]
$$

Let $e_{1}=0, e_{2}=1$ and $e_{3}=2$ be those generalized exponents. Their ramification indices are $n_{e_{1}}=n_{e_{2}}=n_{e_{3}}=1$.

For the operator $M$ we have

$>\quad$ gen_exp $(M, t, x=7)$;

$$
[[-1,0,1, t=x-7]]
$$

So $\bar{e}_{1}=-1, \bar{e}_{2}=0$ and $\bar{e}_{3}=1$ will be the generalized exponents of $\mathrm{M}$ at $x=7$.

$$
\left\{\begin{array}{l}
\bar{e}_{1}-e_{1}=-1-0=-1 \in \mathbb{Z}=\frac{1}{n_{e_{1}}} \mathbb{Z} \\
\bar{e}_{2}-e_{2}=0-1=-1 \in \mathbb{Z}=\frac{1}{n_{e_{2}}} \mathbb{Z} \\
\bar{e}_{3}-e_{3}=1-2=-1 \in \mathbb{Z}=\frac{1}{n_{e_{3}}} \mathbb{Z}
\end{array}\right.
$$

- The generalized exponents of $\mathrm{L}_{12}$ at $x=1$ are

$>\quad$ gen_exp $(\mathrm{L} 12, \mathrm{t}, \mathrm{x}=1)$;

$$
[[0,1,2, t=x-1]]
$$

Let $e_{1}=0, e_{2}=1$ and $e_{3}=2$ be those generalized exponents. Their ramification indices are $n_{e_{1}}=n_{e_{2}}=n_{e_{3}}=1$.

For the operator $\mathrm{M}$ we have

$$
>\quad \operatorname{gen} \_\exp (M, t, x=1) \text {; }
$$

$$
[[0,1,2, t=x-1]]
$$


So $\bar{e}_{1}=0, \bar{e}_{2}=1$ and $\bar{e}_{3}=2$ will be the generalized exponents of $\mathrm{M}$ at $x=0$.

$$
\left\{\begin{array}{l}
\bar{e}_{1}-e_{1}=0-0=0 \in \mathbb{Z}=\frac{1}{n_{e_{1}}} \mathbb{Z}, \\
\bar{e}_{2}-e_{2}=1-1=0 \in \mathbb{Z}=\frac{1}{n_{e_{2}}} \mathbb{Z}, \\
\bar{e}_{3}-e_{3}=2-2=0 \in \mathbb{Z}=\frac{1}{n_{e_{3}}} \mathbb{Z} .
\end{array}\right.
$$

\subsubsection{Relation between Change of Variable Transformation and Generalized Exponents}

The following theorem states how the generalized exponents look like after a change of variables $f$ at the point $p$ such that $f(p)=0$ and $f(p)=\infty$ (i.e. at the zeroes and poles of $f$ ) since we will assume that our differential operator $\mathrm{L}_{0}$ that we want to solve in terms of its solutions has only two singularities: 0 and $\infty$.

Theorem 2.11. Let $\mathrm{L}_{0}, \mathrm{M} \in \mathrm{K}[\partial]$ be two irreducible third-order linear differential operators such that $\mathrm{L}_{0} \stackrel{f}{\longrightarrow}{ }_{C} \mathrm{M}, \quad f \in \mathrm{K} \backslash k$.

(i) Let $p$ be a zero of $f$ with multiplicity $m_{p} \in \mathbb{N}^{*}$ and e a generalized exponent of $\mathrm{L}_{0}$ at $x=0$ with ramification index $n_{e} \in \mathbb{N}^{*}$. Then $p$ is a regular singularity of $\mathrm{M}$ and the generalized exponent of $\mathrm{M}$ at $p$ related to $e$ is

$$
m_{p} \cdot e_{0}-\sum_{i=1}^{n} \sum_{j=-i \cdot m_{p}}^{-1} \frac{j \cdot e_{i} \cdot \sigma^{-i}}{i} \bar{f}_{i, j+i \cdot m_{p}} t_{p}^{j / n_{e}}
$$

where

$$
\left\{\begin{aligned}
& f=t_{p}^{m_{p}} \sum_{j=0}^{+\infty} f_{j} t_{p}^{j}, \quad \text { with } f_{i} \in k \quad \text { and } f_{0} \neq 0, \\
& e=\sum_{i=0}^{n} e_{i} t^{-i} \quad \text { with } b t^{n_{e}}=x, b \in k \backslash\{0\}, n \in \mathbb{N} \text { and } e_{i} \in k, \\
&\left(\sum_{j=0}^{+\infty} f_{j} t_{p}^{j}\right)^{-i / n_{e}}=\sum_{j=0}^{+\infty} \bar{f}_{i, j} t_{p}^{j / n_{e}} \quad \text { with } \bar{f}_{i, j} \in k, i=1, \cdots n \\
& \text { and } \sigma \text { is solution of } X^{n_{e}}-b^{-1}=0 \text { with unknown } X .
\end{aligned}\right.
$$

(ii) Let $p$ be a pole of $f$ with multiplicity $m_{p} \in \mathbb{N}^{*}$ and e a generalized exponent of $\mathrm{L}_{0}$ at $x=0$ with ramification index $n_{e} \in \mathbb{N}^{*}$. Then $p$ is an irregular singularity of $\mathrm{M}$ and the generalized exponent of $\mathrm{M}$ at $p$ related to $e$ is

$$
m_{p} \cdot e_{0}-\sum_{i=1}^{n} \sum_{j=-i \cdot m_{p}}^{-1} \frac{j \cdot e_{i} \cdot \sigma^{-i}}{i} \bar{f}_{i, j+i \cdot m_{p}} t_{p}^{j / n_{e}}
$$


where

$$
\left\{\begin{aligned}
& f=t_{p}^{-m_{p}} \sum_{j=0}^{+\infty} f_{j-m_{p}} t_{p}^{j}, \quad \text { with } f_{j-m_{p}} \in k \text { and } f_{-m_{p}} \neq 0, \\
& e=\sum_{i=0}^{n} e_{i} t^{-i} \text { with } b t^{n_{e}}=t_{\infty}, b \in k \backslash\{0\}, n \in \mathbb{N} \text { and } e_{i} \in k, \\
&\left(\sum_{j=0}^{+\infty} f_{j-m_{p}} t_{p}^{j}\right)^{i / n_{e}}=\sum_{j=0}^{+\infty} \bar{f}_{i, j} t_{p}^{j / n_{e}} \quad \text { with } \bar{f}_{i, j} \in k, i=1, \cdots n \\
& \text { and } \sigma \text { is solution of } X^{n_{e}}-b^{-1}=0 \text { with unknown } X .
\end{aligned}\right.
$$

Proof.

(i) Let $p$ be a zero of $f$ with multiplicity $m_{p} \in \mathbb{N}^{*}$ and $e$ a generalized exponent of $\mathrm{L}_{0}$ at $x=0$ with ramification index $n_{e} \in \mathbb{N}^{*}$. Then $f$ has the representation

$$
f=t_{p}^{m_{p}} \sum_{j=0}^{+\infty} f_{j} t_{p}^{j}, \quad \text { with } f_{i} \in k \text { and } f_{0} \neq 0
$$

and there exists a solution $y(x)$ of $\mathrm{L}_{0}$ at $x=0$ of the form

$$
y(x)=\exp \left(\int \frac{e}{x} d x\right) S(x)
$$

for some Puiseux series $S(x) \in k\left(\left(x^{1 / n_{e}}\right)\right)[\ln (x)]$ with non-zero constant term. Since $e \in k\left[x^{-1 / n_{e}}\right]$ with integer constant term, we can write $e$ in the form

$$
\begin{aligned}
e & =\sum_{i=0}^{n} e_{i} t^{-i} \quad \text { with } b t^{n_{e}}=x, b \in k \backslash\{0\}, n \in \mathbb{N} \text { and } e_{i} \in k \\
& =\sum_{i=0}^{n}\left(e_{i} \cdot \sigma^{-i}\right) x^{-i / n_{e}} \quad \text { with } \sigma \text { solution of } X^{n_{e}}-b^{-1}=0 \text { with unknown } X
\end{aligned}
$$

Hence,

$$
\begin{gathered}
y(x)=\exp \left(\int \frac{\sum_{i=0}^{n}\left(e_{i} \cdot \sigma^{-i}\right) x^{-i / n_{e}}}{x} d x\right) S(x)=\exp \left(\int \sum_{i=0}^{n}\left(e_{i} \cdot \sigma^{-i}\right) x^{-\left(i / n_{e}\right)-1} d x\right) \\
=\exp \left(-\sum_{i=1}^{n} \frac{e_{i} \cdot \sigma^{-i} \cdot n_{e}}{i} x^{-i / n_{e}}\right) x^{e_{0}} S(x) .
\end{gathered}
$$

If we now replace $x$ by $f$ in (2.29), we get a solution $z$ of $\mathrm{M}$ at $p$

$$
z=y(f)=\exp \left(-\sum_{i=1}^{n} \frac{e_{i} \cdot \sigma^{-i} \cdot n_{e}}{i} f^{-i / n_{e}}\right) f^{e_{0}} S(f) .
$$




$$
f^{-i / n_{e}}=t_{p}^{-\frac{i \cdot m_{p}}{n_{e}}}\left(\sum_{j=0}^{+\infty} f_{j} t_{p}^{j}\right)^{-i / n_{e}} \quad \text { and } \quad f^{e_{0}}=t_{p}^{m_{p} \cdot e_{0}}\left(\sum_{j=0}^{+\infty} f_{j} t_{p}^{j}\right)^{e_{0}}
$$

Since $f_{0} \neq 0$ then $\sum_{j=0}^{+\infty} f_{j} t_{p}^{j}$ doesn't vanish at $x=p$. Therefore

$$
\left\{\begin{array}{l}
\left(\sum_{j=0}^{+\infty} f_{j} t_{p}^{j}\right)^{-i / n_{e}}=\sum_{j=0}^{+\infty} \bar{f}_{i, j} t_{p}^{j / n_{e}} \quad \text { with } \bar{f}_{i, j} \in k \\
\left(\sum_{j=0}^{+\infty} f_{j} t_{p}^{j}\right)^{+\infty}=\sum_{j=0}^{+\infty} \widetilde{f}_{j} t_{p}^{j / d} \quad \text { with } \tilde{f}_{j} \in k \text { and } d=\text { denominator of } e_{0}
\end{array}\right.
$$

and we have

$$
\left\{\begin{array}{l}
f^{-i / n_{e}}=t_{p}^{-\frac{i \cdot m_{p}}{n_{e}}} \sum_{j=0}^{+\infty} \bar{f}_{i, j} t_{p}^{j / n_{e}}=\sum_{j=0}^{+\infty} \bar{f}_{i, j} t_{p}^{\left(j-i \cdot m_{p}\right) / n_{e}} \\
f^{e_{0}}=t_{p}^{m_{p} \cdot e_{0}} \sum_{j=0}^{+\infty} \widetilde{f}_{j} t_{p}^{j / d}
\end{array}\right.
$$

Now by replacing (2.32) in (2.30) we get

$$
\begin{aligned}
z & =\exp \left(-\sum_{i=1}^{n} \sum_{j=0}^{+\infty} \frac{e_{i} \cdot \sigma^{-i} \cdot n_{e}}{i} \bar{f}_{i, j} t_{p}^{\left(j-i \cdot m_{p}\right) / n_{e}}\right) S(f) t_{p}^{m_{p} \cdot e_{0}} \sum_{j=0}^{+\infty} \widetilde{f}_{j} t_{p}^{j / d} \\
& =\exp \left(-\sum_{i=1}^{n} \sum_{j=-i \cdot m_{p}}^{+\infty} \frac{e_{i} \cdot \sigma^{-i} \cdot n_{e}}{i} \bar{f}_{i, j+i \cdot m_{p}} t_{p}^{j / n_{e}}\right) S(f) t_{p}^{m_{p} \cdot e_{0}} \sum_{j=0}^{+\infty} \widetilde{f}_{j} t_{p}^{j / d} .
\end{aligned}
$$

By splitting the sum $\sum_{j=-i \cdot m_{p}}^{+\infty}$ in (2.33) into three parts: $-i \cdot m_{p} \leq j \leq-1, j=0$ and $j \geq 1$, we get

$$
\begin{gathered}
z=\exp \left(-\sum_{i=1}^{n} \sum_{j=-i \cdot m_{p}}^{-1} \frac{e_{i} \cdot \sigma^{-i} \cdot n_{e}}{i} \bar{f}_{i, j+i \cdot m_{p}} t_{p}^{j / n_{e}}\right) t_{p}^{m_{p} \cdot e_{0}} \cdot T_{0} T_{1} S(f) \sum_{j=0}^{+\infty} \tilde{f}_{j} t_{p}^{j / d} \\
\text { where }\left\{\begin{array}{l}
T_{0}=\exp \left(-\sum_{i=1}^{n} \frac{e_{i} \cdot \sigma^{-i} \cdot n_{e}}{i} \bar{f}_{i, i \cdot m_{p}}\right), \\
T_{1}=\exp \left(\begin{array}{l}
n \\
\left.-\sum_{i=1}^{n} \sum_{j=1}^{+\infty} \frac{e_{i} \cdot \sigma^{-i} \cdot n_{e}}{i} \bar{f}_{i, j+i \cdot m_{p}} t_{p}^{j / n_{e}}\right) .
\end{array}\right.
\end{array}\right.
\end{gathered}
$$

With $\exp (x)=\sum_{q=0}^{+\infty} \frac{x^{q}}{q !}$ when $x$ tends to zero, we can rewrite $T_{1}$ as power series in $t_{p}$ since $\sum_{i=1}^{n} \sum_{j=1}^{+\infty} \frac{e_{i} \cdot \sigma^{-i} \cdot n_{e}}{i} \bar{f}_{i, j+i \cdot m_{p}} t_{p}^{j / n_{e}}$ also tends to zero when $t_{p}$ tends to zero.

$$
\begin{aligned}
T_{1} & =\sum_{q=0}^{+\infty} \frac{1}{q !}\left[-\sum_{i=1}^{n} \sum_{j=1}^{+\infty} \frac{e_{i} \cdot \sigma^{-i} \cdot n_{e}}{i} \bar{f}_{i, j+i \cdot m_{p}} t_{p}^{j / n_{e}}\right]^{q} \\
& =\sum_{q=0}^{+\infty} a_{q} t_{p}^{q / n_{e}} \quad \text { with } a_{q} \in k \text { and } a_{0}=1
\end{aligned}
$$


By taken $t_{p}$ as the variable and using (2.27), $f$ has no constant term. Adding the fact that $S(x)$ has a non-zero constant term, we conclude that $S(f(x))$ has a non-zero constant term.

$$
\begin{aligned}
f_{0} \neq 0 & \Longrightarrow\left(\sum_{j=0}^{+\infty} f_{j} t_{p}^{j}\right)^{e_{0}} \text { has a non-zero constant term } \\
& \Longrightarrow \sum_{j=0}^{+\infty} \widetilde{f}_{j} t_{p}^{j / d} \text { has a non-zero constant term by }(2.31)
\end{aligned}
$$

Since $t_{p}, t_{p}^{1 / n_{e}}, t_{p}^{1 / n_{e}}, t_{p}^{1 / d} \in k\left(\left(t_{p}^{1 /\left(n_{e} \cdot d\right)}\right)\right), T_{1}, S(f(x))$ and $\sum_{j=0}^{+\infty} \widetilde{f}_{j} t_{p}^{j / d}$ are Puiseux series in $k\left(\left(t_{p}^{1 /\left(n_{e} \cdot d\right)}\right)\right)\left[\ln \left(t_{p}\right)\right]$ with non-zero constant term. With the fact that $T_{0} \neq 0$, otherwise $z$ will be zero, we conclude that

$$
\bar{S}=T_{0} T_{1} S(f) \sum_{j=0}^{+\infty} \widetilde{f}_{j} t_{p}^{j / d} \in k\left(\left(t_{p}^{1 /\left(n_{e} \cdot d\right)}\right)\right)\left[\ln \left(t_{p}\right)\right] \text { with a non-zero constant term }
$$

therefore

$$
\begin{gathered}
z=\exp \left(-\sum_{i=1}^{n} \sum_{j=-i \cdot m_{p}}^{-1} \frac{e_{i} \cdot \sigma^{-i} \cdot n_{e}}{i} \bar{f}_{i, j+i \cdot m_{p}} t_{p}^{j / n_{e}}\right) t_{p}^{m_{p} \cdot e_{0}} \bar{S} \\
=\exp \left(\int\left(-\sum_{i=1}^{n} \sum_{j=-i \cdot m_{p}}^{-1} \frac{e_{i} \cdot \sigma^{-i} \cdot j}{i} \bar{f}_{i, j+i \cdot m_{p}} t_{p}^{j / n_{e}-1}\right) d t_{p}\right) \bar{S} \\
\times \exp \left(\int \frac{m_{p} \cdot e_{0}}{t_{p}} d t_{p}\right) \bar{S} \\
=\exp \left(\int\left(\frac{m_{p} \cdot e_{0}-\sum_{i=1}^{n} \frac{-1}{\sum_{j=-i \cdot m_{p}}^{1}} \frac{e_{i} \cdot \sigma^{-i} \cdot j}{i} \bar{f}_{i, j+i \cdot m_{p}} t_{p}^{j / n_{e}}}{t_{p}}\right) d t_{p}\right) \bar{S}
\end{gathered}
$$

(ii) Let $p$ be a pole of $f$ with multiplicity $m_{p} \in \mathbb{N}^{*}$ and $e$ a generalized exponent of $\mathrm{L}_{0}$ at $x=\infty$ with ramification index $n_{e} \in \mathbb{N}^{*}$. Then $f$ has the representation

$$
f=t_{p}^{-m_{p}} \sum_{j=0}^{+\infty} f_{j-m_{p}} t_{p}^{j}, \quad \text { with } f_{j-m_{p}} \in k \text { and } f_{-m_{p}} \neq 0
$$

and there exists a solution $y(x)$ of $\mathrm{L}_{0}$ at $x=0$ of the form

$$
y(x)=\exp \left(\int \frac{e}{t_{\infty}} d t_{\infty}\right) S\left(t_{\infty}\right)
$$

for some Puiseux series $S\left(t_{\infty}\right) \in k\left(\left(t_{\infty}^{1 / n_{e}}\right)\right)\left[\ln \left(t_{\infty}\right)\right]$ with non-zero constant term. 
Since $e \in k\left[t_{\infty}^{-1 / n_{e}}\right]$ with integer constant term, we can write $e$ in the form

$$
\begin{aligned}
e & =\sum_{i=0}^{n} e_{i} t^{-i / n_{e}} \quad \text { with } b t^{n_{e}}=t_{\infty}, b \in k \backslash\{0\}, n \in \mathbb{N} \text { and } e_{i} \in k \\
& =\sum_{i=0}^{n}\left(e_{i} \cdot \sigma^{-i}\right) t_{\infty}^{-i / n_{e}} \quad \text { with } \sigma \text { solution of } X^{n_{e}}-b^{-1}=0 \text { with unknown } X
\end{aligned}
$$

Hence,

$$
\begin{aligned}
y(x) & =\exp \left(\int \frac{\sum_{i=0}^{n}\left(e_{i} \cdot \sigma^{-i}\right) t_{\infty}^{-i / n_{e}}}{x} d t_{\infty}\right) S\left(t_{\infty}\right) \\
& =\exp \left(\int \sum_{i=0}^{n}\left(e_{i} \cdot a^{-i}\right) t_{\infty}^{-\left(i / n_{e}\right)-1} d t_{\infty}\right) S\left(t_{\infty}\right) \\
& =\exp \left(-\sum_{i=1}^{n} \frac{\left(e_{i} \cdot \sigma^{-i}\right) n_{e}}{i} t_{\infty}^{-i / n_{e}}\right) t_{\infty}^{e_{0}} S\left(t_{\infty}\right) .
\end{aligned}
$$

If we now replace $t_{\infty}$ by $1 / f$ in $(2.37)$ since $t_{\infty}=1 / x$, we get a solution $z$ of $\mathrm{M}$ at $p$

$$
z=y(f)=\exp \left(-\sum_{i=1}^{n} \frac{e_{i} \cdot \sigma^{-i} \cdot n_{e}}{i}\left(\frac{1}{f}\right)^{-i / n_{e}}\right)\left(\frac{1}{f}\right)^{e_{0}} S\left(\frac{1}{f(x)}\right) .
$$

(2.35) gives us

$$
\left\{\begin{array}{l}
\left(\frac{1}{f}\right)^{-i / n_{e}}=t_{p}^{-\frac{i \cdot m_{p}}{n_{e}}}\left(\sum_{j=0}^{+\infty} f_{j-m_{p}} t_{p}^{j}\right)^{i / n_{e}} \\
\left(\frac{1}{f}\right)^{e_{0}}=t_{p}^{m_{p} \cdot e_{0}}\left(\sum_{j=0}^{+\infty} f_{j-m_{p}} t_{p}^{j}\right)^{-e_{0}}
\end{array}\right.
$$

Since $f_{-m_{p}} \neq 0$, it follows that $\sum_{j=0}^{+\infty} f_{j-m_{p}} t_{p}^{j}$ doesn't vanish at $x=p$. Therefore

$$
\left\{\begin{array}{l}
\left(\sum_{j=0}^{+\infty} f_{j-m_{p}} t_{p}^{j}\right)^{i / n_{e}}=\sum_{j=0}^{+\infty} \bar{f}_{i, j} t_{p}^{j / n_{e}} \quad \text { with } \bar{f}_{i, j} \in k, \\
\left(\sum_{j=0}^{+\infty} f_{j-m_{p}} t_{p}^{j}\right)^{-e_{0}}=\sum_{j=0}^{+\infty} \widetilde{f}_{j} t_{p}^{j / d} \text { with } \tilde{f}_{j} \in k \text { and } d=\text { denominator of } e_{0},
\end{array}\right.
$$

and we have

$$
\left\{\begin{array}{l}
\left(\frac{1}{f}\right)^{-i / n_{e}}=t_{p}{ }^{-\frac{i \cdot m_{p}}{n_{e}}} \sum_{j=0}^{+\infty} \bar{f}_{i, j} t_{p}^{j / n_{e}}=\sum_{j=0}^{+\infty} \bar{f}_{i, j} t_{p}^{\left(j-i \cdot m_{p}\right) / n_{e}}, \\
\left(\frac{1}{f}\right)^{e_{0}}=t_{p}^{m_{p} \cdot e_{0}} \sum_{j=0}^{+\infty} \tilde{f}_{j} t_{p}^{j / d}
\end{array}\right.
$$


Now by replacing (2.40) in (2.38) we get

$$
\begin{aligned}
z & =\exp \left(-\sum_{i=1}^{n} \sum_{j=0}^{+\infty} \frac{e_{i} \cdot \sigma^{-i} \cdot n_{e}}{i} \bar{f}_{i, j} t_{p}^{\left(j-i \cdot m_{p}\right) / n_{e}}\right) S\left(\frac{1}{f(x)}\right) t_{p} \frac{m_{p} \cdot e_{0}}{l} \sum_{j=0}^{+\infty} \widetilde{f}_{j} t_{p}^{j / d} \\
& =\exp \left(-\sum_{i=1}^{n} \sum_{j=-i \cdot m_{p}}^{+\infty} \frac{e_{i} \cdot \sigma^{-i} \cdot n_{e}}{i} \bar{f}_{i, j+i \cdot m_{p}} t_{p}^{j / n_{e}}\right) S\left(\frac{1}{f(x)}\right) t_{p}^{m_{p} \cdot e_{0}} \sum_{j=0}^{+\infty} \widetilde{f}_{j} t_{p}^{j / d} .
\end{aligned}
$$

By splitting the sum $\sum_{j=-i \cdot m_{p}}^{+\infty}$ in (2.41) into three parts: $-i m_{p} \leq j \leq-1, j=0$ and $j \geq 1$, we get

$$
\begin{aligned}
& z=\exp \left(-\sum_{i=1}^{n} \sum_{j=-i \cdot m_{p}}^{-1} \frac{e_{i} \cdot \sigma^{-i} \cdot n_{e}}{i} \bar{f}_{i, j+i \cdot m_{p}} t_{p}^{j / n_{e}}\right) t_{p}^{m_{p} \cdot e_{0}} \\
& \times T_{0} T_{1} S\left(\frac{1}{f(x)}\right) \sum_{j=0}^{+\infty} \widetilde{f}_{j} t_{p}^{j / d} \\
& \text { where }\left\{\begin{array}{l}
T_{0}=\exp \left(-\sum_{i=1}^{n} \frac{e_{i} \cdot \sigma^{-i} \cdot n_{e}}{i} \bar{f}_{i, i \cdot m_{p}}\right) \\
T_{1}=\exp \left(-\sum_{i=1}^{n} \sum_{j=1}^{+\infty} \frac{e_{i} \cdot \sigma^{-i} \cdot n_{e}}{i} \bar{f}_{i, j+i \cdot m_{p}} t_{p}^{j / n_{e}}\right) .
\end{array}\right.
\end{aligned}
$$

With $\exp (x)=\sum_{q=0}^{+\infty} \frac{x^{q}}{q !}$ when $x$ tends to zero, we can rewrite $T_{1}$ as power series in $t_{p}$ since $\sum_{i=1}^{n} \sum_{j=1}^{+\infty} \frac{e_{i} \sigma^{-i} n_{e}}{i} \bar{f}_{i, j+i \cdot m_{p}} t_{p}^{j / n_{e}}$ also tends to zero when $t_{p}$ tends to zero.

$$
\begin{aligned}
T_{1} & =\sum_{q=0}^{+\infty} \frac{1}{q !}\left[-\sum_{i=1}^{n} \sum_{j=1}^{+\infty} \frac{e_{i} \cdot \sigma^{-i} \cdot n_{e}}{i} \bar{f}_{i, j+i \cdot m_{p}} t_{p}^{j / n_{e}}\right]^{q} \\
& =\sum_{q=0}^{+\infty} a_{q} t_{p}^{q / n_{e}} \quad \text { with } a_{q} \in k \text { and } a_{0}=1
\end{aligned}
$$

By taking $t_{p}$ as the variable and using (2.35), $1 / f$ has no constant term. Adding the fact that $S(x)$ has a non-zero constant term, we conclude that $S(1 / f(x))$ has a non-zero constant term.

$$
\begin{aligned}
f_{-m_{p}} \neq 0 & \Longrightarrow\left(\sum_{j=0}^{+\infty} f_{j-m_{p}} t_{p}^{j}\right)^{e_{0}} \text { has a non-zero constant term } \\
& \Longrightarrow \sum_{j=0}^{+\infty} \tilde{f}_{j} t_{p}^{j / d} \text { has a non-zero constant term by }(2.39) .
\end{aligned}
$$


Since $t_{p}, t_{p}^{1 / n_{e}}, t_{p}^{1 / n_{e}}, t_{p}^{1 / d} \in k\left(\left(t_{p}^{1 /\left(n_{e} \cdot d\right)}\right)\right), T_{1}, S(1 / f(x))$ and $\sum_{j=0}^{+\infty} \widetilde{f}_{j} t_{p}^{j / d}$ are Puiseux series in $k\left(\left(t_{p}^{1 /\left(n_{e} \cdot d\right)}\right)\right)\left[\ln \left(t_{p}\right)\right]$ with non-zero constant term. With the fact that $T_{0} \neq 0$, otherwise $z$ will be zero, we conclude that

$\bar{S}=T_{0} T_{1} S\left(\frac{1}{f(x)}\right) \sum_{j=0}^{+\infty} \widetilde{f}_{j} t_{p}^{j / d} \in k\left(\left(t_{p}^{1 /\left(n_{e} \cdot d\right)}\right)\right)\left[\ln \left(t_{p}\right)\right]$ with a non-zero constant term,

therefore

$$
\begin{gathered}
z=\exp \left(-\sum_{i=1}^{n} \sum_{j=-i \cdot m_{p}}^{-1} \frac{e_{i} \cdot \sigma^{-i} \cdot n_{e}}{i} \bar{f}_{i, j+i \cdot m_{p}} t_{p}^{j / n_{e}}\right) t_{p}^{m_{p} \cdot e_{0}} \bar{S} \\
=\exp \left(\int\left(-\sum_{i=1}^{n} \sum_{j=-i \cdot m_{p}}^{-1} \frac{e_{i} \cdot \sigma^{-i} \cdot j}{i} \bar{f}_{i, j+i \cdot m_{p}} t_{p}^{j / n_{e}-1}\right) d t_{p}\right) \\
=\exp \left(\int \frac{m_{p} \cdot e_{0}}{t_{p}} d t_{p}\right) \bar{S} \\
\left.=\exp \left(\int \frac{m_{p} \cdot e_{0}-\sum_{i=1}^{n} \frac{\sum_{j=-i \cdot m_{p}}^{-1}}{t_{p}}{ }^{\frac{e_{i} \cdot \sigma^{-i} \cdot j}{i}} \bar{f}_{i, j+i \cdot m_{p}} t_{p}^{j / n_{e}}}{}\right) d t_{p}\right) \bar{S}
\end{gathered}
$$

\section{Example}

Let us take the irreducible third-order linear differential operator $\mathrm{L}_{B B}$ associated to the Bessel square root function $\check{\mathrm{B}}_{\nu}^{2}(x)=\mathrm{B}_{\nu}^{2}(\sqrt{x})$ where $\mathrm{B}_{\nu}$ is the modified Bessel function of the first kind with parameter $\nu=3$.

$$
\begin{array}{rl}
>\mathrm{LBB}:=2 * \mathrm{x}^{\wedge} 2 & * \mathrm{Dx} \wedge 3+6 * \mathrm{x} * \mathrm{Dx} \wedge 2+(-16-2 * \mathrm{x}) * \mathrm{Dx}-1 ; \\
L B B & :=2 x^{2} D x^{3}+6 x D x^{2}+(-16-2 x) D x-1
\end{array}
$$

The generalized exponents of $\mathrm{L}_{B B}$ at $x=0$ are

$>$ gen_exp $(\mathrm{LBB}, \mathrm{t}, \mathrm{x}=0)$;

$$
[[-3,0,3, t=x]]
$$

Let us denote by $e^{\theta}=-3, e^{\phi}=0$ and $e^{\psi}=3$ the generalized exponents of $\mathrm{L}_{B B}$ at $x=0$. For $\alpha \in\{\theta, \phi, \psi\}$ the ramification index of $e^{\alpha}$ is $n_{e^{\alpha}}=1$ and the element $b \in k \backslash\{0\}$ such that $b \cdot t^{n_{e^{\alpha}}}=x$ is $b=1$. Therefore, the solution of $X^{n_{e^{\alpha}}}-b^{-1}=0$ is $\sigma_{\alpha}=1$. By substituting $e^{\alpha}$ in the form $e^{\alpha}=\sum_{i=0}^{n_{\alpha}} e_{i}^{\alpha} t^{-i}$ we have

$$
\left\{\begin{array}{l}
n_{\alpha}=0 \\
e_{0}^{\alpha}=e^{\alpha}
\end{array}\right.
$$

The generalized exponents of $\mathrm{L}_{B B}$ at $x=\infty$ are

$$
>\text { gen_exp(LBB,t,x=infinity); }
$$




$$
\left[\left[1 / 2, t=x^{-1}\right],\left[t^{-1}+1 / 2, t^{2}=x^{-1}\right]\right]
$$

$E^{\theta}=1 / 2$ is a generalized exponent of $\mathrm{L}_{B B}$ at $x=\infty$. Its ramification index is $n_{E^{\theta}}=1$ and the element $b \in k \backslash\{0\}$ such that $b \cdot t^{n_{E^{\theta}}}=t_{\infty}$ is $b=1$. Therefore, the solution of $X^{n_{E^{\theta}}}-b^{-1}=0$ is $\sigma_{\theta}=1$.

$t^{-1}+1 / 2$ with $t^{2}=t_{\infty}$ are generalized exponents of $\mathrm{L}_{B B}$ at $x=\infty$. Their ramification index is 2. The element $b \in k \backslash\{0\}$ such that $b \cdot t^{2}=t_{\infty}$ is $b=1$, and therefore solution of $X^{2}-b^{-1}=0$ are $\sigma_{\phi}=1$ and $\sigma_{\psi}=-1$. Therefore, for $\sigma_{\phi}=1$ (resp. $\sigma_{\psi}=-1$ ) $E^{\phi}=t_{\infty}^{-1 / 2}+1 / 2\left(\right.$ resp. $\left.\quad E^{\psi}=-t_{\infty}^{-1 / 2}+1 / 2\right)$ is a generalized exponent of $\mathrm{L}_{B B}$ at $x=\infty$ with ramification index $n_{E^{\phi}}=2$ (resp. $n_{E^{\psi}}=2$ ). For $\alpha \in\{\theta, \phi, \psi\}$, by substituting $E^{\alpha}$ in the form $E^{\alpha}=\sum_{i=0}^{n_{\alpha}} E_{i}^{\alpha} t^{-i}$ we have

$$
\left\{\begin{array}{l}
n_{\theta}=0, n_{\phi}=1, n_{\psi}=1 \\
E_{0}^{\alpha}=1 / 2 \quad \text { with } \alpha \in\{\theta, \phi, \psi\} \\
E_{1}^{\phi}=1, E_{1}^{\psi}=1
\end{array}\right.
$$

Let $\mathrm{M}$ be the operator coming from $\mathrm{L}_{B B}$ by the change of variable transformation with parameter $f=\frac{(x-1)^{8} x^{6}}{(x-12)^{10}}$.

$>f:=(x-1) \wedge 8 * x \sim 6 /((x-12) \wedge 10) ;$

$$
\begin{aligned}
& f:=\frac{(x-1)^{8} x^{6}}{(x-12)^{10}} \\
&> \text { M:=ChangeOfVariables }(\text { LBB }, \mathrm{f}) ; \\
& M:=(x-1)^{2} x^{2}(x-12)^{13}\left(x^{2}-41 x+18\right)^{2} D x^{3}+3\left(x^{4}-82 x^{3}+575 x^{2}-468 x+216\right) \\
& \times(x-1) x(x-12)^{12}\left(x^{2}-41 x+18\right) D x^{2}-\left(933087744110886912-627170724 x^{17}\right. \\
&-9299742418343559168 x-72791577911076323328 x^{3}+36716049153820459008 x^{2} \\
&+16 x^{22}-2752 x^{21}+183968 x^{20}+92172927 x^{18}+2409383746 x^{16}-6314702496 x^{15} \\
&+541073854638635904 x^{8}-64409641148306304 x^{9}+76208058878030512128 x^{4} \\
&+5730368146930000 x^{10}+30279851895 x^{14}-744912611952 x^{13}+19429306967672 x^{12} \\
&-3332740360025590272 x^{7}-42594780648286715904 x^{5}+14553041176441463040 x^{6} \\
&\left.-5927232 x^{19}-384289295082784 x^{11}\right)(x-12) D x-32(x-1)^{7} x^{5}\left(x^{2}-41 x+18\right)^{5}
\end{aligned}
$$

1. Let us analyse the generalized exponents of $\mathrm{M}$ at the zeros of $f$.

- $x=0$ is a zero of $f$ with order $m_{0}=6$ and the series representation of $f$ at $x=0$ is

$$
\begin{aligned}
& >\operatorname{series}(\mathrm{f}, \mathrm{x}=0,9) ; \\
& \quad \frac{x^{6}}{61917364224}-\frac{43 x^{7}}{371504185344}+\frac{3127 x^{8}}{8916100448256}+O\left(x^{9}\right)
\end{aligned}
$$

Hence $f$ has the form $x^{6} \sum_{j=0}^{+\infty} f_{j} x^{j}$ with $f_{j} \in k$ and $f_{0} \neq 0$. The generalized exponents of $\mathrm{M}$ at $x=0$ are

$>\quad$ gen_exp $(M, t, x=0)$; 


$$
[[-18,0,18, t=x]] \text {. }
$$

Let $\overline{e^{\theta}}=-18, \overline{e^{\phi}}=0$ and $\overline{e^{\psi}}=18$ we have

$$
\left\{\begin{array}{l}
m_{0} \cdot e_{0}^{\theta}=6 \times(-3)=-18=\overline{e^{\theta}}, \\
m_{0} \cdot e_{0}^{\phi}=6 \times 0=0=\overline{e^{\phi}} \\
m_{0} \cdot e_{0}^{\psi}=6 \times 3=18=\overline{e^{\psi}} .
\end{array}\right.
$$

Since $n_{\alpha}=0 \quad \forall \alpha \in\{\theta, \phi, \psi\}$, our Theorem 2.11 is satisfied.

- $x=1$ is a zero of $f$ with order $m_{1}=8$ and the series representation of $f$ at $x=1$ is

$>\operatorname{series}(f, x=1,10)$;

$$
\frac{(x-1)^{8}}{25937424601}+\frac{76(x-1)^{9}}{285311670611}+O\left((x-1)^{10}\right)
$$

Hence $f$ has the form $(x-1)^{8} \sum_{j=0}^{+\infty} f_{j} t_{1}^{j}$ with $f_{j} \in k$ and $f_{0} \neq 0$. The generalized exponents of $\mathrm{M}$ at $x=1$ are

$$
>\quad \operatorname{gen} \_\exp (\mathrm{M}, \mathrm{t}, \mathrm{x}=1) \text {; }
$$

$$
[[-24,0,24, t=x-1]]
$$

Let $\overline{e^{\theta}}=-24, \overline{e^{\phi}}=0$ and $\overline{e^{\psi}}=24$ we have

$$
\left\{\begin{array}{l}
m_{1} \cdot e_{0}^{\theta}=8 \times(-3)=-24=\overline{e^{\theta}} \\
m_{1} \cdot e_{0}^{\phi}=8 \times 0=0=\overline{e^{\phi}}, \\
m_{1} \cdot e_{0}^{\psi}=8 \times 3=24=\overline{e^{\psi}} .
\end{array}\right.
$$

Since $n_{\alpha}=0 \quad \forall \alpha \in\{\theta, \phi, \psi\}$, our Theorem 2.11 is satisfied.

2. Let us analyse the generalized exponents of $\mathrm{M}$ at the poles of $f$.

- $x=12$ is a pole of $f$ with order $m_{12}=10$ and the series representation of $f$ at $x=12$ is

$$
\begin{aligned}
& >\quad \operatorname{series}(\mathrm{f}, \mathrm{x}=12) ; \\
& 640072188923904(x-12)^{-10}+785543140952064(x-12)^{-9} \\
& +447543588019968(x-12)^{-8}+156886659198720(x-12)^{-7} \\
& +37804890836592(x-12)^{-6}+6624387287496(x-12)^{-5} \\
& +O\left((x-12)^{-4}\right)
\end{aligned}
$$

Hence $f$ has the form $(x-12)^{-10} \sum_{j=0}^{+\infty} f_{j-10} t_{12}^{j}$ with $f_{j} \in k$ and $f_{-10} \neq 0$. Let

$$
\left(\sum_{j=0}^{+\infty} f_{j-10} t_{12}^{j}\right)^{i / n_{E^{\alpha}}}=\sum_{j=0}^{+\infty} \bar{f}_{i, j} t_{12}^{j / n_{E^{\alpha}}} \quad \text { with } i \in \mathbb{N}, i \geq 1 \text { and } \alpha \in\{\theta, \phi, \psi\} .
$$

Since $n_{\alpha} \in\{0,1\} \quad \forall \alpha \in\{\theta, \phi, \psi\}$, our $i$ will be in $\{0,1\}$. For $i=1$, we have 


$$
\begin{aligned}
& >\operatorname{series}((\mathrm{f} *(\mathrm{x}-12)-10) \sim(1 / 2), \mathrm{x}=12) ; \\
& -160997760+15524784 x+4081572(x-12)^{2}+595969(x-12)^{3} \\
& +52196(x-12)^{4}+2742(x-12)^{5}+O\left((x-12)^{6}\right)
\end{aligned}
$$

So $\bar{f}_{1,0}=-160997760, \bar{f}_{1,2}=15524784, \bar{f}_{1,4}=4081572, \bar{f}_{1,6}=595969$, $\bar{f}_{1,8}=52196$, and $\bar{f}_{1, j}=0 \quad \forall j \in\{1,3,5,7,9\}$. The generalized exponents of M at $x=12$ are

$$
\begin{aligned}
& >\quad \text { gen_exp }(\mathrm{M}, \mathrm{t}, \mathrm{x}=12) ; \\
& \quad\left[[5, t=x-12],\left[5-2383876 t^{-2}-124198272 t^{-4}-252996480 t^{-5}\right.\right. \\
& \left.\quad-104392 t^{-1}-24489432 t^{-3}, t=x-12\right],\left[2383876 t^{-2}+104392 t^{-1}\right. \\
& \left.\left.\quad+252996480 t^{-5}+124198272 t^{-4}+24489432 t^{-3}+5, t=x-12\right]\right]
\end{aligned}
$$

Let

$$
\left\{\begin{array}{l}
\overline{E^{\theta}}=5, \\
\overline{E^{\phi}}=-252996480 t^{-5}-104392 t^{-1}-124198272 t^{-4}-24489432 t^{-3} \\
\quad-2383876 t^{-2}+5 \\
\overline{E^{\psi}}=252996480 t^{-5}+104392 t^{-1}+124198272 t^{-4}+24489432 t^{-3} \\
+2383876 t^{-2}+5 .
\end{array}\right.
$$

We have

$$
\left\{\begin{array}{l}
m_{12} \cdot E_{0}^{\theta}=10 \times 1 / 2=\overline{E^{\theta}}, \\
m_{12} \cdot E_{0}^{\phi}-\sum_{j=-10}^{-1} j \cdot E_{1}^{\phi} \cdot \sigma_{\phi} \cdot \bar{f}_{1, j+10} t_{12}^{j / n_{E^{\phi}}}=\overline{E^{\phi}} \\
m_{12} \cdot E_{0}^{\psi}-\sum_{j=-10}^{-1} j \cdot E_{1}^{\psi} \cdot \sigma_{\psi} \cdot \bar{f}_{1, j+10} t_{12}^{j / n_{E^{\psi}}}=\overline{E^{\psi}} .
\end{array}\right.
$$

Since $n_{\theta}=0, n_{\phi}=1$ and $n_{\psi}=1$, our Theorem 2.11 is satisfied.

- $x=\infty$ is a pole of $f$ with order $m_{\infty}=4$ and the series representation of $f$ at $x=\infty$ is

$$
\begin{aligned}
& >\quad \operatorname{series}(\mathrm{f}, \mathrm{x}=\text { infinity) } \\
& x^{4}+112 x^{3}+6988 x^{2}+320104 x+12000070+389761048 x^{-1} \\
& \quad+11353950076 x^{-2}+303501512344 x^{-3}+7565103474817 x^{-4} \\
& +177920465247480 x^{-5}+O\left(x^{-6}\right)
\end{aligned}
$$

Hence $f$ has the form $t_{\infty}^{-4} \sum_{j=0}^{+\infty} f_{j-4} t_{\infty}^{j}$ with $f_{j} \in k$ and $f_{-4} \neq 0$. Let

$$
\left(\sum_{j=0}^{+\infty} f_{j-4} t_{\infty}^{j}\right)^{i / n_{E^{\alpha}}}=\sum_{j=0}^{+\infty} \bar{f}_{i, j} t_{\infty}^{j / n_{E^{\alpha}}} \quad \text { with } i \in \mathbb{N}, i \geq 1 \text { and } \alpha \in\{\theta, \phi, \psi\}
$$

Since $n_{\alpha} \in\{0,1\} \quad \forall \alpha \in\{\theta, \phi, \psi\}$, our $i$ will be in $\{0,1\}$. For $i=1$, we have

$$
\begin{aligned}
& >\operatorname{series}((\mathrm{f} *(1 / \mathrm{x}) \sim 4) \wedge(1 / 2), \mathrm{x}=\inf \text { inity }, 5) ; \\
& 1+56 x^{-1}+1926 x^{-2}+52196 x^{-3}+1222321 x^{-4}+O\left(x^{-5}\right)
\end{aligned}
$$


So $\bar{f}_{1,0}=1, \bar{f}_{1,2}=56$ and $\bar{f}_{1, j}=0 \quad \forall j \in\{1,3\}$. The generalized exponents of M at $x=\infty$ are

$>\quad \operatorname{gen} \_\exp (\mathrm{M}, \mathrm{t}, \mathrm{x}=$ infinity $)$;

$\left[\left[2, t=x^{-1}\right],\left[-4 t^{-2}-112 t^{-1}+2, t=x^{-1}\right],\left[4 t^{-2}+112 t^{-1}+2, t=x^{-1}\right]\right]$

Let

$$
\left\{\begin{array}{l}
\overline{E^{\theta}}=2, \\
\overline{E^{\phi}}=4 t^{-2}+112 t^{-1}+2, \\
\overline{E^{\psi}}=-4 t^{-2}-112 t^{-1}+2 .
\end{array}\right.
$$

We have

$$
\left\{\begin{array}{l}
m_{\infty} \cdot E_{0}^{\theta}=2=\overline{E^{\theta}}, \\
m_{\infty} \cdot E_{0}^{\phi}-\sum_{j=-4}^{-1} j \cdot E_{1}^{\phi} \cdot \sigma_{\phi} \cdot \bar{f}_{1, j+4} t_{\infty}^{j / n_{E^{\phi}}}=\overline{E^{\phi}}, \\
m_{\infty} \cdot E_{0}^{\psi}-\sum_{j=-4}^{-1} j \cdot E_{1}^{\psi} \cdot \sigma_{\psi} \cdot \bar{f}_{1, j+4} t_{\infty}^{j / n_{E} \psi}=\overline{E^{\psi}} .
\end{array}\right.
$$

Since $n_{\theta}=0, n_{\phi}=1$ and $n_{\psi}=1$, our Theorem 2.11 is satisfied.

Let us now consider another operator $\mathrm{M}$ coming also from $\mathrm{L}_{B B}$ above but with the change of variable parameter $f=\frac{(x-3)^{2}}{x^{10}}$.

$$
\begin{aligned}
>\mathrm{f}:= & (\mathrm{x}-3) \sim 2 / \mathrm{x} \wedge 10 ; \\
\qquad \mathrm{M}:= & \text { ChangeOfVariables }(\mathrm{LBB}, \mathrm{f}) ; \\
M:= & (x-3)^{2} x^{13}(4 x-15)^{2} D x^{3}+3\left(4 x^{2}-30 x+45\right)(x-3) x^{12}(4 x-15) D x^{2} \\
& -x\left(1822500-3159000 x-872640 x^{3}+2276100 x^{2}+1820475 x^{10}+9200 x^{14}\right. \\
& \left.-138000 x^{13}+776250 x^{12}-1941030 x^{11}-21504 x^{5}+1024 x^{6}+187776 x^{4}\right) D x \\
& +4(4 x-15)^{5}(x-3)
\end{aligned}
$$

1. Let us analyse the generalized exponents of $\mathrm{M}$ at the zeros of $f$.

- $x=3$ is a zero of $f$ with order $m_{3}=2$ and the series representation of $f$ at $x=3$ is

$$
\begin{array}{r}
>\operatorname{series}(\mathrm{f}, \mathrm{x}=3) ; \\
\frac{(x-3)^{2}}{59049}-\frac{10(x-3)^{3}}{177147}+\frac{55(x-3)^{4}}{531441} \\
-\frac{220(x-3)^{5}}{1594323}+O\left((x-3)^{6}\right)
\end{array}
$$

Hence $f$ has the form $(x-3)^{2} \sum_{j=0}^{+\infty} f_{j} t_{3}^{j}$ with $f_{j} \in k$ and $f_{0} \neq 0$. The generalized exponents of $\mathrm{M}$ at $x=3$ are

$>$ gen_exp $(M, t, x=3)$;

$$
[[-6,0,6, t=x-3]]
$$


Let $\overline{e^{\theta}}=-6, \overline{e^{\phi}}=0$ and $\overline{e^{\psi}}=6$ we have

$$
\left\{\begin{array}{l}
m_{3} \cdot e_{0}^{\theta}=2 \times(-3)=-6=\overline{e^{\theta}}, \\
m_{3} \cdot e_{0}^{\phi}=2 \times 0=0=\overline{e^{\phi}} \\
m_{3} \cdot e_{0}^{\psi}=2 \times 3=6=\overline{e^{\psi}} .
\end{array}\right.
$$

Since $n_{\alpha}=0 \forall \alpha \in\{\theta, \phi, \psi\}$, our Theorem 2.11 is satisfied.

- $x=\infty$ is a zero of $f$ with order $m_{\infty}=8$ and the series representation of $f$ at $x=\infty$ is

$>\operatorname{series}(\mathrm{f}, \mathrm{x}=$ infinity, 12$)$;

$$
x^{-8}-6 x^{-9}+9 x^{-10}
$$

Hence $f$ has the form $t_{\infty}^{8} \sum_{j=0}^{+\infty} f_{j} t_{\infty}^{j}$ with $f_{j} \in k$ and $f_{0} \neq 0$. The generalized exponents of $\mathrm{M}$ at $x=\infty$ are

$$
>\quad \operatorname{gen} \_\exp (\mathrm{M}, \mathrm{t}, \mathrm{x}=\text { infinity }) \text {; }
$$

$$
\left[\left[-24,0,24, t=x^{-1}\right]\right]
$$

Let $\overline{e^{\theta}}=-24, \overline{e^{\phi}}=0$ and $\overline{e^{\psi}}=24$ we have

$$
\left\{\begin{array}{l}
m_{\infty} \cdot e_{0}^{\theta}=8 \times(-3)=-24=\overline{e^{\theta}} \\
m_{\infty} \cdot e_{0}^{\phi}=8 \times 0=0=\overline{e^{\phi}} \\
m_{\infty} \cdot e_{0}^{\psi}=8 \times 3=24=\overline{e^{\psi}} .
\end{array}\right.
$$

Since $n_{\alpha}=0 \forall \alpha \in\{\theta, \phi, \psi\}$, our Theorem 2.11 is satisfied.

2. Let us analyse the generalized exponents of $M$ at the poles of $f$.

The only pole of $f$ is $x=0$ with order $m_{0}=10$ and the series representation of $f$ at $x=0$ is

$>\operatorname{series}(f, x=0)$;

$$
x^{-8}-6 x^{-9}+9 x^{-10}
$$

Hence $f$ has the form $x^{-10} \sum_{j=0}^{+\infty} f_{j-10} x^{j}$ with $f_{j} \in k$ and $f_{-10} \neq 0$. Let

$$
\left(\sum_{j=0}^{+\infty} f_{j-10} x^{j}\right)^{i / n_{E^{\alpha}}}=\sum_{j=0}^{+\infty} \bar{f}_{i, j} x^{j / n_{E^{\alpha}}} \quad \text { with } i \in \mathbb{N}, i \geq 1 \text { and } \alpha \in\{\theta, \phi, \psi\} .
$$

Since $n_{\alpha} \in\{0,1\} \quad \forall \alpha \in\{\theta, \phi, \psi\}$, our $i$ will be in $\{0,1\}$. For $i=1$, we have

$>\operatorname{series}\left(f * x^{\wedge} 5, x=0\right)$;

$$
x-3
$$

So $\bar{f}_{1,0}=3, \bar{f}_{1,2}=-1$ and $\bar{f}_{1, j}=0 \quad \forall j \in\{1,3,4,5,6,7,8,9\}$. The generalized exponents of $\mathrm{M}$ at $x=0$ are

$>\quad \operatorname{gen} \_\exp (\mathrm{M}, \mathrm{t}, \mathrm{x}=0)$;

$$
\left[[5, t=x],\left[-30 t^{-5}+8 t^{-4}+5, t=x\right],\left[30 t^{-5}-8 t^{-4}+5, t=x\right]\right]
$$


Let $\overline{E^{\theta}}=5, \overline{E^{\phi}}=30 x^{-5}-8 t^{-4}+5$ and $\overline{E^{\psi}}=-30 x^{-5}+8 t^{-4}+5$. We have

$$
\left\{\begin{array}{l}
m_{0} \cdot E_{0}^{\theta}=5=\overline{E^{\theta}} \\
m_{0} \cdot E_{0}^{\phi}-\sum_{j=-10}^{-1} j \cdot E_{1}^{\phi} \cdot \sigma_{\phi} \cdot \bar{f}_{1, j+5} x^{j / n_{E^{\phi}}}=\overline{E^{\phi}} \\
m_{0} \cdot E_{0}^{\psi}-\sum_{j=-10}^{-1} j \cdot E_{1}^{\psi} \cdot \sigma_{\psi} \cdot \bar{f}_{1, j+5} x^{j / n_{E^{\psi}}}=\overline{E^{\psi}} .
\end{array}\right.
$$

Since $n_{\theta}=0, n_{\phi}=1$ and $n_{\psi}=1$, our Theorem 2.11 is satisfied. 


\section{Chapter 3}

\section{Steps to Find Solutions}

In this chapter, all the results are ours, except in

- the p-curvature test where some results come from Katz [24], van der Put [30], Quan Yuan [42], Cluzeau and van Hoeij [10], and Alin Bostan, Xavier Caruso and Eric Schost [4];

- the gauge equivalence which contains an algorithm from van Hoeij, van der Put and Michael Singer (see [17] and [32]).

From the previous chapter, we know how two irreducible third-order linear differential operators can be connected and some modifications that can appear when we move from the solution space of one of them to the other. In this chapter, we try

1. first to clarify our method of solving a third-order differential operator in terms of solutions of another third-order differential operator. That will be done by considering some specific transformations (connections) learnt previously between them: change of variables, exp-product and gauge transformations. L will be our given third-order differential operator and $\mathrm{L}_{0}$ the third-order differential operator that we need to solve $\mathrm{L}$ in terms of its solutions. Hence, by the previous studies on those transformations in the last chapter, we will have

$$
\mathrm{L}_{0} \stackrel{f}{\longrightarrow} C \mathrm{M} \longrightarrow E G \mathrm{~L}
$$

where $\mathrm{M}$ is a differential operator and $r, r_{2}, r_{1}, r_{0}, f \in \mathrm{K}$ the parameters of the transformations. By taking $y$ as solution of $\mathrm{L}_{0}$, the solutions of $\mathrm{L}$ will be written in the form

$$
\exp \left(\int r d x\right)\left(r_{2} y(f(x))^{\prime \prime}+r_{1} y(f(x))^{\prime}+r_{0} y(f(x))\right) .
$$

In this work, the solution $y$ of $\mathrm{L}_{0}$ will be one of the following functions: Bessel square root function $\check{\mathrm{B}}_{\nu}^{2}(x)=\mathrm{B}_{\nu}^{2}(\sqrt{x})$ where $\mathrm{B}_{\nu}$ is the Bessel function, ${ }_{2} F_{2},{ }_{1} F_{2},{ }_{0} F_{2}$ hypergeometric functions and the square of the ${ }_{1} F_{1}$ hypergeometric function.

2. Then we give some steps and main tools to find the parameters of the transformations between the operators $\left(\mathrm{L}_{0}\right.$ and $\left.\mathrm{L}\right)$ :

(a) the first step will be to find the change of variable parameter $(f)$. That will be done mainly by some studies on the generalized exponents of $\mathrm{L}_{1} \mathrm{~L}_{0}$ and $\mathrm{M}$ (if those transformations exist) at their singularities which will help us to find the zeroes and poles of $f$, with also their associated multiplicity orders. 
(b) If $f$ is known (or equivalently $\mathrm{M}$ ), then the next step will be to find the expproduct and gauge transformations. That will be done also by some studies on the generalized exponents of $\mathrm{L}$ and $\mathrm{M}$ at their singularities. The p-curvature test can be good in order to reduce the number of candidates for the expproduct and gauge transformations. But we will not use it since it uses one of Grothendieck's conjectures which is not yet proved.

Let $k$ be an extension field of $\mathbb{Q}$ which is algebraically closed and has characteristic zero. Let $\mathrm{K}=k(x)$ be the field of rational functions in $x$. All of our differential operators are irreducible and belong to $\mathrm{K}[\partial]$. For $n \in \mathbb{N} \backslash\{0\}$ and $a$ an element of $\mathrm{K}=k(x)$ or not, when we will talk about $a$ modulo $\frac{1}{n} \mathbb{Z}$, that will means $a$ modulo an additive element of $\frac{1}{n} \mathbb{Z}$

\subsection{Meaning of our Problem}

With what we have learnt in the last chapter, we can state what we mean by solving a differential operator in terms of a particular function.

Definition 3.1. Assuming $y$ is a solution of a differential operator $\mathrm{L}_{0}$, we say that we can solve a differential operator $\mathrm{L}$ in terms of $y$ when we can find the transformations

$$
\mathrm{L}_{0} \stackrel{f}{\longrightarrow}_{C} \mathrm{M} \longrightarrow E G \mathrm{~L}
$$

where $\mathrm{M}$ is a differential operator. That is: the solutions of $\mathrm{L}$ can be written in the following form

$$
\exp \left(\int r d x\right)\left(r_{2} y(f(x))^{\prime \prime}+r_{1} y(f(x))^{\prime}+r_{0} y(f(x))\right)
$$

with $r, r_{2}, r_{1}, r_{0}, f \in \mathrm{K}$ (parameters of transformations).

We will concentrated in this thesis on the determination of those parameters of transformation.

In our work, we solve irreducible third-order linear differential equations in terms of those functions:

- Bessel square root functions $\check{\mathrm{B}}_{\nu}^{2}(x)=\mathrm{B}_{\nu}^{2}(\sqrt{x})$ where $\mathrm{B}_{\nu}^{2}$ is the Bessel square functions of parameter $\nu$, with associated differential operators

$$
\mathrm{L}_{\check{B}_{\nu}^{2}}=2 x^{2} \partial^{3}+6 x \partial^{2}+\left(2-2 x-2 \nu^{2}\right) \partial-1
$$

- the ${ }_{2} F_{2}\left(\begin{array}{c}a_{1}, a_{2} \\ b_{1}, b_{2}\end{array} \mid x\right)$ hypergeometric functions, with associated differential operators

$$
\mathrm{L}_{22}=x^{2} \partial^{3}+x\left(1+b_{1}+b_{2}-x\right) \partial^{2}+\left(b_{1} b_{2}-x\left(1+a_{1}+a_{2}\right)\right) \partial-a_{1} a_{2},
$$


- the ${ }_{1} F_{2}\left(\begin{array}{c}a_{1} \\ b_{1}, b_{2}\end{array} \mid x\right)$ hypergeometric functions, with associated differential operators $\mathrm{L}_{12}=x^{2} \partial^{3}+x\left(1+b_{1}+b_{2}\right) \partial^{2}+\left(b_{1} b_{2}-x\right) \partial-a_{1}$,

- the ${ }_{0} F_{2}\left(\begin{array}{c|c}- & \\ b_{1}, b_{2} & x\end{array}\right)$ hypergeometric functions, with associated differential operators $\mathrm{L}_{02}=x^{2} \partial^{3}+x\left(1+b_{1}+b_{2}\right) \partial^{2}+b_{1} b_{2} \partial-1$,

- the ${ }_{1} F_{1}^{2}\left(\begin{array}{l}a \\ b\end{array} \mid x\right)$ functions, with associated differential operators

$$
\mathrm{L}_{11}^{2}=x^{2} \partial^{3}+3 x(-x+b) \partial^{2}-\left(-2 x^{2}+4 x(a+b)-b(2 b-1)\right) \partial-2 a(-2 x+2 b-1)
$$

where $a_{1}, a_{2}, b_{1}, b_{2}, a, b$ are constants called hypergeometric parameters, and $\nu$ is a constant called Bessel parameter.

Therefore, $\mathrm{L}_{0}$ can be $\mathrm{L}_{\breve{B}_{\nu}^{2}}$ or $\mathrm{L}_{22}$ or $\mathrm{L}_{12}$ or $\mathrm{L}_{02}$ or $\mathrm{L}_{11}^{2}$. All those third-order operators are irreducible and have singularities just at 0 and $\infty$.

Remarks 3.2. 1. For a given hypergeometric function, one can generate the associated differential equation using Theorem 1.18. That is how we got the differential equation satisfied by ${ }_{1} F_{1},{ }_{0} F_{2},{ }_{1} F_{2}$ and ${ }_{2} F_{2}$.

2. For a given hypergeometric function, one can use algebraic operations to develop differential equation satisfied by its powers, its derivative, the power of its derivative and by its composition with a algebraic function (see [39] and [33]).

3. In general, the product of two solutions of a second-order differential equation satisfies a fourth order differential equation. However, the square of a solution of differential equation of order two can satisfy a differential equation of order three. That is the case for the differential equation satisfied by the functions $\check{\mathrm{B}}_{\nu}^{2}=\mathrm{B}_{\nu}^{2}(\sqrt{x})$ and ${ }_{1} F_{1}^{2}$ (see [39] and [33]).

Lemma 3.3. Let $\mathrm{L}_{0} \in\left\{\mathrm{L}_{\breve{B}_{\nu}^{2}}, \mathrm{~L}_{22}, \mathrm{~L}_{12}, \mathrm{~L}_{02}, \mathrm{~L}_{11}^{2}\right\}$. Then $x=0$ is a regular singularity of $\mathrm{L}_{0}$ and $x=\infty$ is an irregular singularity of $\mathrm{L}_{0}$.

Proof. Let $\mathrm{L}_{0} \in\left\{\mathrm{L}_{\check{B}_{\nu}^{2}}, \mathrm{~L}_{22}, \mathrm{~L}_{12}, \mathrm{~L}_{02}, \mathrm{~L}_{11}^{2}\right\}$. By the representation of $\mathrm{L}_{0}, \mathrm{~L}_{0}$ has two singularities: 0 and $\infty$.

1. For the point $x=0$.

(a) If $\mathrm{L}_{0}=\mathrm{L}_{\check{B}_{\nu}^{2}}$ then, by using Maple, the generalized exponents of $\mathrm{L}_{0}$ at $x=0$ are

$$
-\nu, \quad 0, \quad \nu
$$

i. For $\nu \neq 0, \mathrm{~L}_{0}$ has at same time positive and negative constant generalized exponents at $x=0$. So $x=0$ is a regular singularity of $\mathrm{L}_{0}$. 
ii. For $\nu=0$ all the generalized exponents of $\mathrm{L}_{0}$ at $x=0$ are zero. By checking, using Maple, if $\mathrm{L}_{0}$ has at this point a logarithmic solution, we get

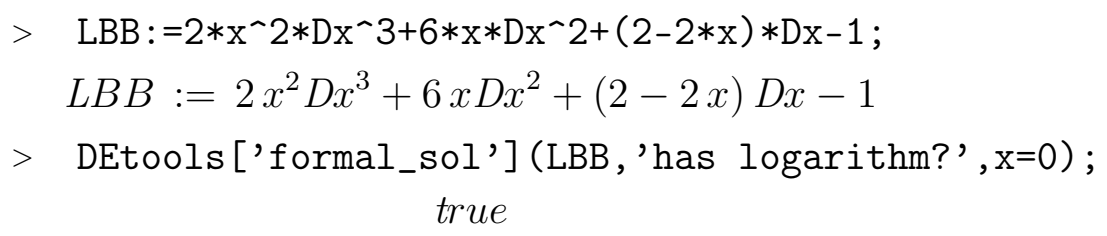

So at $x=0$ we have a logarithmic solution of $\mathrm{L}_{0}$. Therefore $x=0$ is a regular singularity of $\mathrm{L}_{0}$.

(b) If $\mathrm{L}_{0} \in\left\{\mathrm{L}_{22}, \mathrm{~L}_{12}, \mathrm{~L}_{02}, \mathrm{~L}_{11}^{2}\right\}$ then, by using Maple, the generalized exponents of $\mathrm{L}_{0}$ at $x=0$ are

i. for $\mathrm{L}_{0} \in\left\{\mathrm{L}_{22}, \mathrm{~L}_{12}, \mathrm{~L}_{02}\right\}$

$$
1-b_{1}, \quad 0, \quad 1-b_{2}
$$

ii. for $\mathrm{L}_{0}=\mathrm{L}_{11}^{2}$

$$
0, \quad 1-b, \quad 2(1-b)
$$

where $b, b_{1}$ and $b_{2}$ are the lower parameters of the hypergeometric function associated to $\mathrm{L}_{0}$. Since we don't have the variable $t_{0}=x$ in the expression of the generalized exponents, $x=0$ can be either a regular point or an apparent singularity or a regular singularity of $\mathrm{L}_{0}$.

For $x=0$ to be an apparent singularity or a regular point of $\mathrm{L}_{0}$, it is necessary that $1-b, 1-b_{1}, 1-b_{2} \in \mathbb{N}$. Since the lower parameter(s) of a hypergeometric function cannot be zero $\left(b \neq 0, b_{1} \neq 0, b_{2} \neq 0\right)$, that means $b=b_{1}=b_{2}=1$. Hence all the generalized exponents of $\mathrm{L}_{0}$ at $x=0$ are zero. Using the fact that at a regular point the generalized exponents are 0,1 and 2 (see corollary 1.41), $x=0$ cannot be a regular point. By checking, using Maple, if $\mathrm{L}_{0}$ has at $x=0$ a logarithmic solution, the answer is "true". For example when $\mathrm{L}_{0}=\mathrm{L}_{12}$ with upper parameter $a_{1}=1$

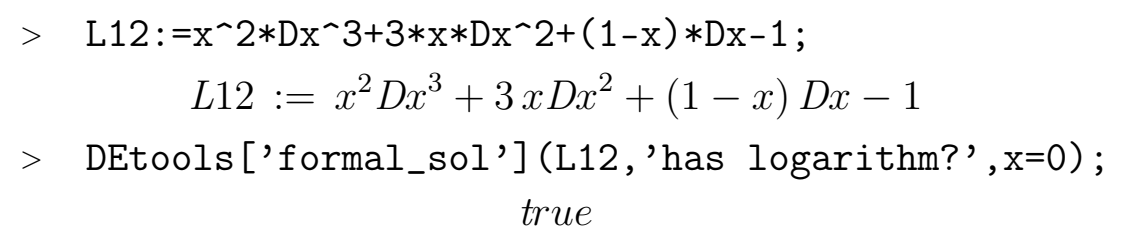

Hence $x=0$ is neither an apparent singularity nor a regular point of $\mathrm{L}_{0}$. So $x=0$ is a regular singularity of $\mathrm{L}_{0}$.

2. For the point $x=\infty$, by using Maple to compute the generalized exponents, we have at least one non-constant generalized exponent of $\mathrm{L}_{0}$ at this point. So $x=\infty$ is an irregular singularity of $\mathrm{L}_{0}$.

Remark 3.4. The necessary and sufficient condition for $\mathrm{L}_{0}$ to have a logarithmic solution at $x=0$ is that

1. for $\mathrm{L}_{0}=\mathrm{L}_{\check{B}_{\nu}^{2}}$ we have $\nu \in \mathbb{Z}$, 
2. for $\mathrm{L}_{0} \in\left\{\mathrm{L}_{22}, \mathrm{~L}_{12}, \mathrm{~L}_{02}\right\}$ we have $b_{1} \in \mathbb{Z}$ or $b_{2} \in \mathbb{Z}$ or $b_{1}-b_{2} \in \mathbb{Z}$ or $\left(2 b_{1}, 2 b_{2} \in\right.$ $\mathbb{Z}$ with $b 1 \cdot b 2<0)$, and

3. for $\mathrm{L}_{0}=\mathrm{L}_{11}^{2}$ we have $b \in \mathbb{Z}$.

Let $y$ be a solution of the differential operator $\mathrm{L}_{0}$, and $\mathrm{L}$ a given irreducible third-order linear differential operator that we want to solve in terms of $y$. There are two steps to find $y$ type solutions of L. The first step is to find the middle operator M (i.e the change of variables $f$ ). If $\mathrm{M}$ (or equivalently $f$ ) is known, then the next step is to find the map from $M$ to L: This step is called the equivalence of differential operators.

\subsection{The first step to solve our problem}

This step is to find the change of variable parameters $f$ such that

$$
\mathrm{L}_{0} \stackrel{f}{\longrightarrow} C \mathrm{M} \text {. }
$$

Here we will just give capital tools that can help us to find this parameter.

If $y$ is a solution of $\mathrm{L}_{0}$ then $y(f(x))$ is a solution of M. If $y$ has singularity at $p_{0}$, it is obvious that $y(f(x))$ has a singularity at those points $x$ such that $f(x)=p_{0}$. Since in our case $\mathrm{L}_{0}$ has only singularities at 0 and $\infty$, if $y$ has a singularity at $p_{0}$ then $p_{0} \in\{0, \infty\}$ (singularity of a solution of an operator is always singularity of this operator). Therefore, a singularity of $\mathrm{M}$ which is not an apparent singularity belong to the set formed by the zeroes $(f(x)=0)$ and poles $(f(x)=\infty)$ of $f$ :

- Any regular singularity of $\mathrm{M}$ is a zero of $f$, but the converse is not always true.

- Poles of $f$ are irregular singularities of M.

\subsubsection{Observations}

The main way to find a rational function (here $f$ ) is to find its zeroes and its poles. We are showing here how the singularities of $\mathrm{L}$ can be related to the zeroes and poles of $f$.

\section{case 1: $\mathrm{L}=\mathrm{M}$}

That is when we have no exp-product and gauge transformations:

$$
\mathrm{L}_{0} \stackrel{f}{\rightarrow} \mathrm{L}_{\mathrm{L}}
$$

The poles of $f$ are always the irregular singularities of L but for a zero of $f$ it can become either a regular singularity or an apparent singularity or a regular point of L.

(a) All the zeroes of $f$ are regular singularities of L.

Example: Let us take $\mathrm{L}_{0}$ to be the Bessel square root operator $\mathrm{L}_{\check{B}_{\nu}^{2}}$ with parameter $\nu=3$ and the change of variable parameter $f=\frac{(x-1)^{8} x^{6}}{(x-12)^{10}}$.

$>\mathrm{LBB}:=2 * \mathrm{x}^{\wedge} 2 * \mathrm{Dx} \wedge 3+6 * \mathrm{x} * \mathrm{Dx} \wedge 2+(-2 * \mathrm{x}-16) * \mathrm{Dx}-1$;

$$
L B B:=2 x^{2} D x^{3}+6 x D x^{2}+(-2 x-16) D x-1
$$




$$
\begin{aligned}
& >\mathrm{f}:=(\mathrm{x}-1) \sim 8 * \mathrm{x}^{\wedge} 6 /(\mathrm{x}-12) \sim 10 \\
& f:=\frac{(x-1)^{8} x^{6}}{(x-12)^{10}} \\
& >\mathrm{L}:=\text { ChangeOfVariables }(\mathrm{LBB}, \mathrm{f}) ; \\
L:= & (x-1)^{2} x^{2}(x-12)^{13}\left(x^{2}-41 x+18\right)^{2} D x^{3}+3 x\left(x^{4}-82 x^{3}+575 x^{2}-468 x\right. \\
& +216)(x-1)(x-12)^{12}\left(x^{2}-41 x+18\right) D x^{2}-\left(-42594780648286715904 x^{5}\right. \\
& +933087744110886912-9299742418343559168 x+36716049153820459008 x^{2} \\
& +14553041176441463040 x^{6}+76208058878030512128 x^{4}+5730368146930000 x^{10} \\
& +16 x^{22}-2752 x^{21}+183968 x^{20}-5927232 x^{19}+30279851895 x^{14}+92172927 x^{18} \\
& -627170724 x^{17}+2409383746 x^{16}-6314702496 x^{15}-3332740360025590272 x^{7} \\
& -72791577911076323328 x^{3}+541073854638635904 x^{8}-64409641148306304 x^{9} \\
& \left.-744912611952 x^{13}+19429306967672 x^{12}-384289295082784 x^{11}\right)(x-12) D x \\
& -32 x^{5}(x-1)^{7}\left(x^{2}-41 x+18\right)^{5}
\end{aligned}
$$

The zeroes of $f$ are $x=0$ and $x=1$, and the poles of $f$ are $x=12$ and $x=\infty$.

Next we compute all the singularities of $\mathrm{L}$ which are different from $\infty$ :

$>\quad\{$ solve $(\operatorname{coeff}(\mathrm{L}, \mathrm{Dx}, 3), \mathrm{x})\}$;

$$
\left\{0,1,12, \frac{41}{2}-1 / 2 \sqrt{1609}, \frac{41}{2}+1 / 2 \sqrt{1609}\right\}
$$

The generalized exponents of $\mathrm{L}$ at its singularities are

$$
\begin{aligned}
& >\quad \text { gen_exp }(L, t, x=0) \text {; } \\
& {[[-18,0,18, t=x]]} \\
& >\quad \operatorname{gen} \_\exp (\mathrm{L}, \mathrm{t}, \mathrm{x}=1) \text {; } \\
& {[[-24,0,24, t=x-1]]} \\
& >\quad \text { gen_exp }(\mathrm{L}, \mathrm{t}, \mathrm{x}=12) \text {; } \\
& {\left[[5, t=x-12],\left[-252996480 t^{-5}-104392 t^{-1}-124198272 t^{-4}-24489432 t^{-3}\right.\right.} \\
& \left.-2383876 t^{-2}+5, t=x-12\right],\left[252996480 t^{-5}+104392 t^{-1}+124198272 t^{-4}\right. \\
& \left.\left.+24489432 t^{-3}+2383876 t^{-2}+5, t=x-12\right]\right] \\
& >\quad \operatorname{gen} \_\exp (\mathrm{L}, \mathrm{t}, \mathrm{x}=41 / 2-(1 / 2) * \operatorname{sqrt}(1609)) \text {; } \\
& {\left[\left[0,2,4, t=x-\frac{41}{2}+1 / 2 \sqrt{1609}\right]\right]} \\
& >\quad \operatorname{gen} \_\exp (\mathrm{L}, \mathrm{t}, \mathrm{x}=41 / 2+(1 / 2) * \operatorname{sqrt}(1609)) \text {; } \\
& {\left[\left[0,2,4, t=x-\frac{41}{2}-1 / 2 \sqrt{1609}\right]\right]} \\
& >\text { gen_exp(L,t, } \mathrm{x}=\text { infinity); } \\
& {\left[\left[2, t=x^{-1}\right],\left[4 t^{-2}+112 t^{-1}+2, t=x^{-1}\right],\left[-4 t^{-2}-112 t^{-1}+2, t=x^{-1}\right]\right]}
\end{aligned}
$$

Hence $x=0$ and $x=1$ are regular singularities of $\mathrm{L}$ since all of their generalized exponents are constant and not all of them have the same sign. So all the zeroes of $f$ are regular singularities of L. The only irregular singularities of L are $x=12$ and 
$x=\infty$ since $\mathrm{L}$ has at those points at least one non-constant generalized exponents. They correspond to the poles of $f$.

(b) A zero of $f$ can become an apparent singularity of L.

Example: Let us take $\mathrm{L}_{0}$ to be the operator $\mathrm{L}_{02}$ coming from the hypergeometric function ${ }_{0} F_{2}\left(\begin{array}{c|c}- \\ 3 / 4,1 / 2\end{array} \mid x\right)$, and the change of variable parameter $f=2(x-1)^{8}$.

$>\quad \mathrm{L} 02:=\mathrm{x}^{\wedge} 2 * \mathrm{Dx}^{\wedge} 3+(9 / 4) * \mathrm{x} * \mathrm{Dx}^{\wedge} 2+(3 / 8) * \mathrm{Dx}-1$;

$$
L 02:=x^{2} D x^{3}+9 / 4 x D x^{2}+3 / 8 D x-1
$$

$>f:=2 *(x-1) \sim 8$

$$
f:=2(x-1)^{8}
$$

$>\mathrm{L}:=$ ChangeOfVariables $(\mathrm{LO} 2, \mathrm{f})$;

$$
L:=(x-1)^{2} D x^{3}+(-3 x+3) D x^{2}+3 D x-1024(x-1)^{7}
$$

$x=1$ is a zero of $f$. Let us see which kind of point $x=1$ is for L. First we have to see if $x=1$ is a singularity or a regular point of $\mathrm{L}$ :

$>\{\operatorname{solve}(\operatorname{coeff}(\mathrm{L}, \mathrm{Dx}, 3), \mathrm{x})\}$;

Hence $x=1$ is a singularity of L.

$>\quad \operatorname{gen} \_\exp (\mathrm{L}, \mathrm{t}, \mathrm{x}=1)$;

$$
[[0,2,4, t=x-1]]
$$

So $x=1$ can be either an apparent singularity or a regular singularity of L. Since all the generalized exponents at $x=1$ are positive, for $x=1$ to be a regular singularity, $\mathrm{L}$ must have at this point a logarithmic solution.

$>$ DEtools['formal_sol'] (L, 'has logarithm?', $x=1$ );

$$
\text { false }
$$

Therefore $x=1$ is an apparent singularity of $\mathrm{L}$.

(c) A zero of $f$ can become a regular point of $\mathrm{L}$.

Example: Let us take $\mathrm{L}_{0}$ as in $(b)$ above but now with the change of variable parameter $f=2(x-1)^{4}$.

$>f:=2 *(x-1) \sim 4$;

$$
f:=2(x-1)^{4}
$$

$>\quad \mathrm{L}:=$ ChangeOfVariables $(\mathrm{L} 02, \mathrm{f})$;

$$
L:=D x^{3}+128-128 x
$$

$x=1$ is a zero of $f$. Let us see which kind of point $x=1$ is for L. First we have to see if $x=1$ is a singularity or a regular point of L:

$>\quad\{$ solve $(\operatorname{coeff}(\mathrm{L}, \mathrm{Dx}, 3), \mathrm{x})\}$;

So $x=1$ is not a singularity of $\mathrm{L}$. That can also be seen by computing the generalized exponents of L at $x=1$ : 
$>\quad \operatorname{gen} \_\exp (\mathrm{L}, \mathrm{t}, \mathrm{x}=1)$;

$$
[[0,1,2, t=x-1]]
$$

Therefore $x=1$ is a regular point of $\mathrm{L}$.

\section{case 2: $\quad L \neq M$}

That is when both exp-product and a gauge transformation are involved:

$$
\mathrm{L}_{0} \stackrel{f}{\longrightarrow}_{C} \mathrm{M} \longrightarrow{ }_{E G} \mathrm{~L} .
$$

(a) Regular singularity of L can be a regular singularity of $\mathrm{M}$.

Example: Let us take $\mathrm{L}_{0}$ to be the Bessel square root operator $\mathrm{L}_{\check{B}_{\nu}^{2}}$ with parameter $\nu=3$ and

- the change of variable parameter $f=4(x-1)^{2}$,

- the exp-product parameter $r=\frac{(x-3)^{5}}{(x-7)^{2}}$, and

- the gauge parameters $r_{0}=1, r_{1}=0$ and $r_{2}=0$.

$>\mathrm{LBB}:=2 * \mathrm{x}^{\wedge} 2 * \mathrm{Dx}^{\wedge} 3+6 * \mathrm{x} * \mathrm{Dx}^{\wedge} 2+(-2 * \mathrm{x}-16) * \mathrm{Dx}-1$;

$$
L B B:=2 x^{2} D x^{3}+6 x D x^{2}+(-2 x-16) D x-1
$$

$>\mathrm{f}:=4 *(\mathrm{x}-1)^{\sim} 2$;

$$
f:=4(x-1)^{2}
$$

$>\quad M:=$ ChangeOfVariables $(\mathrm{LBB}, \mathrm{f})$;

$$
M:=D x^{3}(x-1)^{2}+(-3+3 x) D x^{2}+\left(-16 x^{2}+32 x-51\right) D x-16 x+16
$$

$>\mathrm{r}:=(\mathrm{x}-3) \wedge 5 /(\mathrm{x}-7) \sim 2$;

$>$ L1:=ExpProduct $(\mathrm{M}, \mathrm{r})$;

$$
r:=\frac{(x-3)^{5}}{(x-7)^{2}}
$$

$$
\begin{aligned}
L 1:= & (x-7)^{6}(x-1)^{2} D x^{3}-3\left(674 x^{2}-634 x+194+x^{6}-16 x^{5}+105 x^{4}\right. \\
& \left.-360 x^{3}\right)(x-7)^{4}(x-1) D x^{2}+\left(-66075-410476 x+1637220 x^{2}\right. \\
& -1850976 x^{5}+872012 x^{6}+2763364 x^{4}-2756132 x^{3}-295032 x^{7}-96 x^{11} \\
& \left.+71670 x^{8}-12240 x^{9}+1398 x^{10}+3 x^{12}\right)(x-7)^{2} D x-30962009 \\
& +74560847 x-6765252 x^{2}-712520204 x^{5}+612617908 x^{6}+569424702 x^{4} \\
& -253119160 x^{3}-x^{17}+47 x^{16}-1036 x^{15}-390079508 x^{7}+190709494 x^{8} \\
& -72915454 x^{9}+21964884 x^{10}+14220 x^{14}-136068 x^{13}+962598 x^{12} \\
& -5207528 x^{11} \\
>\quad \text { r0 } & :=1 ;
\end{aligned}
$$

$$
r 0:=1
$$

$>\mathrm{r} 1:=0$

$$
r 1:=0
$$

$>r 2:=0$

$$
r 2:=0
$$




$$
\begin{aligned}
>\mathrm{L}:= & \text { GaugeTransf }(\mathrm{L} 1, \mathrm{r} 0, \mathrm{r} 1, \mathrm{r} 2) ; \\
L:= & (x-1)^{5}(x-7)^{12} D x^{3}-3\left(674 x^{2}-634 x+194+x^{6}-16 x^{5}+105 x^{4}\right. \\
& \left.-360 x^{3}\right)(x-1)^{4}(x-7)^{10} D x^{2}+\left(-66075-410476 x+1637220 x^{2}\right. \\
& +872012 x^{6}+2763364 x^{4}-2756132 x^{3}-295032 x^{7}-96 x^{11}+3 x^{12} \\
& \left.-1850976 x^{5}+71670 x^{8}-12240 x^{9}+1398 x^{10}\right)(x-1)^{3}(x-7)^{8} D x \\
& -\left(30962009-74560847 x+6765252 x^{2}+712520204 x^{5}-612617908 x^{6}\right. \\
& -569424702 x^{4}+253119160 x^{3}+x^{17}-47 x^{16}+1036 x^{15}+390079508 x^{7} \\
& -190709494 x^{8}+72915454 x^{9}-21964884 x^{10}-14220 x^{14}+136068 x^{13} \\
& \left.-962598 x^{12}+5207528 x^{11}\right)(x-1)^{3}(x-7)^{6}
\end{aligned}
$$

Let us see which kind of point $x=1$ is for the operators $\mathrm{L}$ and M:

$>\quad \operatorname{gen} \_\exp (M, t, x=1)$;

$$
>\operatorname{gen} \_\exp (\mathrm{L}, \mathrm{t}, \mathrm{x}=1) ; \begin{aligned}
& {[[-6,0,6, t=x-1]]} \\
& {[[-6,0,6, t=x-1]]}
\end{aligned}
$$

Hence $x=1$ is a regular singularity of $\mathrm{L}$ and $\mathrm{M}$ since all its generalized exponents, in the case of L and M, are constant and all of them don't have the same sign.

(b) Regular singularity of $\mathrm{L}$ can be a regular point of $\mathrm{M}$.

Example: Let us take $\mathrm{L}_{0}$ to be the operator $\mathrm{L}_{02}$ coming from the hypergeometric function ${ }_{0} F_{2}\left(\begin{array}{c|c}- \\ 3 / 4,1 / 2\end{array} \mid x\right)$, and

- the change of variable parameter $f=\frac{2}{(x-1)^{4}}$,

- the exp-product parameter $r=\frac{3 / 7}{x}$, and

- the gauge parameters $r_{0}=x-1, r_{1}=0$ and $r_{2}=0$.

$>\quad \mathrm{L} 02:=\mathrm{x}^{\wedge} 2 * \mathrm{Dx}^{\wedge} 3+(9 / 4) * \mathrm{x} * \mathrm{Dx} \wedge 2+(3 / 8) * \mathrm{Dx}-1$;

$$
L 02:=x^{2} D x^{3}+9 / 4 x D x^{2}+3 / 8 D x-1
$$

$>\mathrm{f}:=2 /(\mathrm{x}-1) \sim 4$;

$$
f:=2(x-1)^{-4}
$$

$>\quad M:=$ ChangeOfVariables $($ L02, f);

$$
M:=(x-1)^{7} D x^{3}+6(x-1)^{6} D x^{2}+6(x-1)^{5} D x+128
$$

$>$ L1:=ExpProduct $(\mathrm{M}, \mathrm{r})$;

$$
\begin{aligned}
L 1:= & 343 x^{3}(x-1)^{7} D x^{3}+147 x^{2}(11 x+3)(x-1)^{6} D x^{2} \\
& +42 x\left(22 x^{2}+12 x+15\right)(x-1)^{5} D x-2310 x-630 x^{5} \\
& +510+420 x^{6}+1470 x^{4}-132 x^{7}+4032 x^{2}+40544 x^{3}
\end{aligned}
$$

$>\mathrm{r} 0:=\mathrm{x}-1$

$$
r 0:=x-1
$$


$>r 1:=0$

$$
r 1:=0
$$

$>\mathrm{r} 2:=0$

$$
r 2:=0
$$

$>\mathrm{L}:=$ GaugeTransf $(\mathrm{L} 1, \mathrm{r} 0, \mathrm{r} 1, \mathrm{r} 2)$;

$$
\begin{aligned}
L:= & 343 x^{3}(x-1)^{8} D x^{3}+147 x^{2}(4 x+3)(x-1)^{7} D x^{2} \\
& -126 x(2 x+5)(x-1)^{7} D x+2\left(3465 x^{2}-1470 x+255\right. \\
& \left.+2625 x^{4}+60 x^{7}-105 x^{6}-630 x^{5}+17752 x^{3}\right)(x-1)
\end{aligned}
$$

Let us see which kind of point $x=\infty$ is for the operators $\mathrm{L}$ and $\mathrm{M}$ :

$>\quad$ gen_exp $(M, t, x=i n f i n i t y)$;

$$
\left[\left[0,1,2, t=x^{-1}\right]\right]
$$

$>$ gen_exp(L,t,x=infinity);

$$
\left[\left[-\frac{10}{7},-3 / 7,4 / 7, t=x^{-1}\right]\right]
$$

Hence $x=\infty$ is a regular singularity of $\mathrm{L}$ since all its generalized exponents are constants and all of them are not integers. But $x=\infty$ is a regular point of M.

(c) Apparent singularity of L can be a regular singularity of $\mathrm{M}$.

Example: Let us take $\mathrm{L}_{0}$ to be the Bessel square root operator $\mathrm{L}_{\check{B}_{\nu}^{2}}$ with parameter $\nu=1 / 3$ and

- the change of variable parameter $f=\frac{4}{(x-1)^{6}}$,

- the exp-product parameter $r=-\frac{3}{x}$, and

- the gauge parameters $r_{0}=1, r_{1}=0$ and $r_{2}=0$.

$>\mathrm{LBB}:=2 * \mathrm{x}^{\wedge} 2 * \mathrm{Dx} \wedge 3+6 * \mathrm{x} * \mathrm{Dx}^{\wedge} 2+(-2 * \mathrm{x}+16 / 9) * \mathrm{Dx}-1$;

$$
L B B:=2 x^{2} D x^{3}+6 x D x^{2}+\left(-2 x+\frac{16}{9}\right) D x-1
$$

$>f:=4 /(x-1) \sim 6$

$$
f:=4(x-1)^{-6}
$$

$>\quad M:=$ ChangeOf Variables $(L B B, f)$;

$$
\begin{aligned}
M:= & (x-1)^{9} D x^{3}+3(x-1)^{8} D x^{2}-3\left(x^{6}-6 x^{5}+15 x^{4}\right. \\
& \left.-20 x^{3}+15 x^{2}-6 x+49\right)(x-1) D x+432
\end{aligned}
$$

$>r:=-3 / x$

$$
r:=-3 x^{-1}
$$

$>$ L1:=ExpProduct $(\mathrm{M}, \mathrm{r})$;

$$
\begin{aligned}
L 1:= & x^{3}(x-1)^{9} D x^{3}+3 x^{2}(4 x-3)(x-1)^{8} D x^{2}+3 x\left(11 x^{8}-84 x^{7}+279 x^{6}\right. \\
& \left.-526 x^{5}+615 x^{4}-456 x^{3}+161 x^{2}-54 x+6\right)(x-1) D x-6+72 x \\
& +81 x^{2}+1701 x^{5}-1197 x^{6}-1575 x^{4}+945 x^{3}+531 x^{7}-135 x^{8}+15 x^{9} \\
>\quad \mathrm{r} 0 & :=1 ;
\end{aligned}
$$




$$
\begin{aligned}
& r 0:=1 \\
& >r 1:=0 \text {; } \\
& r 1:=0 \\
& >\mathrm{r} 2:=0 \text {; } \\
& r 2:=0 \\
& >\mathrm{L}:=\text { GaugeTransf }(\mathrm{L} 1, \mathrm{r} 0, \mathrm{r} 1, \mathrm{r} 2) \text {; } \\
& L:=x^{3}(x-1)^{18} D x^{3}+3 x^{2}(4 x-3)(x-1)^{17} D x^{2}+3 x\left(11 x^{8}-84 x^{7}+279 x^{6}\right. \\
& \left.-526 x^{5}+615 x^{4}-456 x^{3}+161 x^{2}-54 x+6\right)(x-1)^{10} D x+3(-2+24 x \\
& \left.+27 x^{2}+567 x^{5}-399 x^{6}-525 x^{4}+315 x^{3}+177 x^{7}-45 x^{8}+5 x^{9}\right)(x-1)^{9}
\end{aligned}
$$

Let us see which kind of point $x=\infty$ is for the operators L and M:

$$
\begin{gathered}
>\text { gen_exp }(M, t, x=\operatorname{infinity~}) ; \\
{\left[\left[-2,0,2, t=x^{-1}\right]\right]} \\
>\text { gen_exp }(\mathrm{L}, \mathrm{t}, \mathrm{x}=\inf \text { inity }) ; \\
{\left[\left[1,3,5, t=x^{-1}\right]\right]}
\end{gathered}
$$

Hence $x=\infty$ is a regular singularity of $\mathrm{M}$ since all its generalized exponents are constants and all of them don't have the same sign. But $x=\infty$ can be a regular singularity or an apparent singularity of L (we have excluded the case of $x=\infty$ to be a regular point of $\mathrm{L}$ since its generalized exponents are not $0,1,2$ ). To have an clear idea about $x=\infty$ we need to check, using Maple, if L has at this point a logarithmic solution:

\section{$>$ DEtools['formal_sol'] (L, 'has logarithm?', x=infinity); false}

So L doesn't have logarithm solution at $x=\infty$. Therefore $x=\infty$ is an apparent singularity of L.

(d) Irregular singularities of L can be a regular singularity of M.

Example: Let us take $\mathrm{L}_{0}$ to be the Bessel square root operator $\mathrm{L}_{\check{B}_{\nu}^{2}}$ with parameter $\nu=3$ and

- the change of variable parameter $f=4(x-1)^{2}$,

- the exp-product parameter $r=\frac{(x-3)^{5}}{(x-1)^{2}}$, and

- the gauge parameters $r_{0}=1, r_{1}=0$ and $r_{2}=0$.

$>\mathrm{LBB}:=2 * \mathrm{x}^{\wedge} 2 * \mathrm{Dx}^{\wedge} 3+6 * \mathrm{x} * \mathrm{Dx}^{\wedge} 2+(-2 * \mathrm{x}-16) * \mathrm{Dx}-1$;

$$
L B B:=2 x^{2} D x^{3}+6 x D x^{2}+(-2 x-16) D x-1
$$

$>\mathrm{f}:=4 *(\mathrm{x}-1) \sim 2$;

$$
f:=4(x-1)^{2}
$$

$>\quad M:=$ ChangeOfVariables $(\mathrm{LBB}, \mathrm{f})$;

$$
\begin{aligned}
M & :=(x-1)^{2} D x^{3}+(-3+3 x) D x^{2}+\left(-16 x^{2}+32 x-51\right) D x-16 x+16 \\
& >\quad \mathrm{r}:=(\mathrm{x}-3)-5 /(\mathrm{x}-1)-2 ;
\end{aligned}
$$


$>$ L1: $=\operatorname{ExpProduct}(\mathrm{M}, \mathrm{r})$;

$$
r:=\frac{(x-3)^{5}}{(x-1)^{2}}
$$

$$
\begin{aligned}
L 1:= & (x-1)^{6} D x^{3}-3\left(404 x-242+x^{5}-15 x^{4}+90 x^{3}-270 x^{2}\right)(x-1)^{4} D x^{2} \\
& +\left(175881-586306 x+880339 x^{2}-183498 x^{5}+51015 x^{6}+458069 x^{4}\right. \\
& \left.-783836 x^{3}-9720 x^{7}+1215 x^{8}-90 x^{9}+3 x^{10}\right)(x-1)^{2} D x+14218567 \\
& -70983755 x+165535207 x^{2}-175940275 x^{5}+97936689 x^{6}+239499317 x^{4} \\
& +239499317 x^{4}-239206503 x^{3}-x^{15}-42052279 x^{7}+14039831 x^{8}-945 x^{13} \\
& -3644249 x^{9}+729387 x^{10}+45 x^{14}+12285 x^{12}-110553 x^{11} \\
>\quad & \mathrm{r} 0:=1 ; \\
>\quad & \mathrm{r} 1:=0 ; \\
>\quad & \quad r 2:=0 ; \\
>\quad & \mathrm{L}:=\text { GaugeTransf }(\mathrm{L} 1, \mathrm{r} 0, \mathrm{r} 1, \mathrm{r} 2) ; \\
L= & (x-1)^{12} D x^{3}-3\left(404 x-242+x^{5}-15 x^{4}+90 x^{3}-270 x^{2}\right)(x-1)^{10} D x^{2} \\
& +\left(175881-586306 x+880339 x^{2}-183498 x^{5}+51015 x^{6}+458069 x^{4}\right. \\
& -\left(-14218567-783836 x^{3}-9720 x^{7}+1215 x^{8}-90 x^{9}+3 x^{10}\right)(x-1)^{8} D x \\
& +70983755 x-165535207 x^{2}+175940275 x^{5}-97936689 x^{6}-239499317 x^{4} \\
& -729387 x^{10}+239206503 x^{3}+x^{15}+42052279 x^{7}-14039831 x^{8}+3644249 x^{9} \\
& \left.-45 x^{14}+945 x^{13}-12285 x^{12}+110553 x^{11}\right)(x-1)^{6}
\end{aligned}
$$

Let us see which kind of point $x=1$ is for the operators $\mathrm{L}$ and $\mathrm{M}$ :

$>\quad \operatorname{gen} \_\exp (\mathrm{M}, \mathrm{t}, \mathrm{x}=1)$;

$$
\begin{gathered}
{[[-6,0,6, t=x-1]]} \\
>\quad \operatorname{gen} \_\exp (\mathrm{L}, \mathrm{t}, \mathrm{x}=1) ; \\
{\left[\left[74-32 t^{-1}, 80-32 t^{-1}, 86-32 t^{-1}, t=x-1\right]\right]}
\end{gathered}
$$

Hence $x=1$ is a regular singularity of $\mathrm{M}$ since all its generalized exponents are constants and all of them don't have the same sign. The generalized exponents of $\mathrm{L}$ at $x=1$ contain the parameter $t$, so $x=1$ is an irregular singularity of L.

(e) Irregular singularities of $\mathrm{L}$ can be a regular point of $\mathrm{M}$.

Example: Let us take $\mathrm{L}_{0}$ to be the Bessel square root operator $\mathrm{L}_{\check{B}_{\nu}^{2}}$ as in $(d)$ with the same change of variable parameter $f$ and the same gauge parameters. For the exp-product parameter, let us take $r=\frac{(x-3)^{5}}{(x-7)^{2}}$.

$>\quad r:=(x-3) \sim 5 /(x-7) \sim 2 ;$

$>\quad \mathrm{L} 1:=\operatorname{ExpProduct}(\mathrm{M}, \mathrm{r})$;

$$
r:=\frac{(x-3)^{5}}{(x-7)^{2}}
$$




$$
\begin{aligned}
L 1:= & (x-7)^{6}(x-1)^{2} D x^{3}-3\left(674 x^{2}-634 x+194+x^{6}-16 x^{5}+105 x^{4}-360 x^{3}\right) \\
& \times(x-7)^{4}(x-1) D x^{2}+\left(-66075-410476 x+1637220 x^{2}-1850976 x^{5}+3 x^{12}\right. \\
& +2763364 x^{4}-2756132 x^{3}-295032 x^{7}+71670 x^{8}-12240 x^{9}+1398 x^{10}-96 x^{11} \\
& \left.+872012 x^{6}\right)(x-7)^{2} D x-30962009-1036 x^{15}-390079508 x^{7}+74560847 x \\
& -6765252 x^{2}-712520204 x^{5}+612617908 x^{6}+569424702 x^{4}-253119160 x^{3} \\
& -x^{17}+47 x^{16}+190709494 x^{8}-72915454 x^{9}+21964884 x^{10}-136068 x^{13} \\
& +14220 x^{14}+962598 x^{12}-5207528 x^{11} \\
& >\quad r 0:=1 ; \\
& >\quad r 1:=0 ; \\
& \quad r 1:=0 \\
& >\text { r } 2:=0 ; \\
& >\quad \mathrm{L}:=\text { GaugeTransf }(\mathrm{L} 1, \mathrm{r} 0, \mathrm{r} 1, \mathrm{r} 2) ; \\
L= & (x-1)^{5}(x-7)^{12} D x^{3}-3\left(674 x^{2}-634 x+194+x^{6}-16 x^{5}+105 x^{4}-360 x^{3}\right) \\
& \times(x-1)^{4}(x-7)^{10} D x^{2}+\left(-66075-410476 x+1637220 x^{2}+1398 x^{10}+3 x^{12}\right. \\
+ & 872012 x^{6}+2763364 x^{4}-2756132 x^{3}-295032 x^{7}+71670 x^{8}-12240 x^{9}-96 x^{11} \\
- & \left.1850976 x^{5}\right)(x-1)^{3}(x-7)^{8} D x-\left(30962009-612617908 x^{6}-569424702 x^{4}\right. \\
- & 74560847 x+6765252 x^{2}+712520204 x^{5}+253119160 x^{3}+x^{17}-47 x^{16} \\
+ & 1036 x^{15}+390079508 x^{7}-190709494 x^{8}+72915454 x^{9}-21964884 x^{10} \\
- & \left.14220 x^{14}+136068 x^{13}-962598 x^{12}+5207528 x^{11}\right)(x-1)^{3}(x-7)^{6} \\
& +
\end{aligned}
$$

Let us see which kind of point $x=7$ is for the operators $\mathrm{L}$ and $\mathrm{M}$ :

$>\quad\{\operatorname{solve}(\operatorname{coeff}(\mathrm{M}, \mathrm{Dx}, 3), \mathrm{x})\}$;

So $x=7$ is a regular point of $\mathrm{M}$. That can also be seen by computing the generalized exponents of $\mathrm{M}$ at $x=7$

$>\quad$ gen_exp $(M, t, x=7)$;

$$
[[0,1,2, t=x-7]]
$$

For the operator $\mathrm{L}$ we have

$>\quad \operatorname{gen} \_\exp (\mathrm{L}, \mathrm{t}, \mathrm{x}=7)$;

$$
\left[\left[1280+1024 t^{-1}, 1281+1024 t^{-1}, 1282+1024 t^{-1}, t=x-7\right]\right]
$$

The generalized exponents of $\mathrm{L}$ at $x=7$ contain the parameter $t$, so $x=7$ is an irregular singularity of L.

(f) Apparent singularities of $\mathrm{L}$ can be a regular point of $\mathrm{M}$.

Example: Let us take $\mathrm{L}_{0}$ to be the operator $\mathrm{L}_{02}$ as in $(b)$ with the change of variable parameter $f=2(x-1)^{4}$ and the same gauge parameters. For the exp-product parameter, let us take $r=x-3$.

$>f:=2 *(x-1) \sim 4$; 


$$
\begin{aligned}
& \quad f:=2(x-1)^{4} \\
& >\quad \mathrm{M}:=\text { ChangeOfVariables }(\mathrm{L} 02, \mathrm{f}) ; \\
& M:=D x^{3}+128-128 x \\
& >\quad \mathrm{r}:=\mathrm{x}-3 ; \quad r:=x-3 \\
& >\quad \mathrm{L} 1:=\text { ExpProduct }(\mathrm{M}, \mathrm{r}) ; \\
& L 1:=D x^{3}+(9-3 x) D x^{2}+3(x-2)(x-4) D x+146-152 x-x^{3}+9 x^{2} \\
& >\quad \mathrm{r} 0:=\mathrm{x}-1 ; \quad r 0:=x-1 \\
& >\quad \mathrm{r} 1:=0 ; \\
& >\quad \mathrm{r} 2:=0 ; \\
& >\quad \mathrm{L}:=\text { GaugeTransf }(\mathrm{L} 1, \mathrm{r} 0, \mathrm{r} 1, \mathrm{r} 2) ; \\
& L=(x-1)^{6} D x^{3}-3(x-2)^{2}(x-1)^{5} D x^{2}+3(x-2)\left(x^{3}-6 x^{2}+11 x-8\right)(x \\
& \quad-1)^{4} D x-\left(972 x^{2}-654 x^{3}+185 x^{4}+x^{6}-680 x-12 x^{5}+194\right)(x-1)^{3}
\end{aligned}
$$

Let us see which kind of point $x=1$ is for the operators $\mathrm{L}$ and M:

$>\quad\{\operatorname{solve}(\operatorname{coeff}(\mathrm{M}, \mathrm{Dx}, 3), \mathrm{x})\}$;

So $x=1$ is a regular point of $\mathrm{M}$. That can also be seen by computing the generalized exponents of $\mathrm{M}$ at $x=1$

$>\quad \operatorname{gen} \_\exp (M, t, x=1)$;

$$
[[0,1,2, t=x-1]]
$$

For the operator L we have

$>\quad \operatorname{gen} \_\exp (\mathrm{L}, \mathrm{t}, \mathrm{x}=1)$;

$$
[[1,2,3, t=x-1]]
$$

Hence $x=1$ can be a regular singularity or an apparent singularity of $\mathrm{L}$ (we have excluded the case of $x=1$ to be a regular point of $\mathrm{L}$ since its generalized exponents are not $0,1,2)$. To have a clear idea about $x=1$ we need to check, using Maple, if $\mathrm{L}$ has at this point a logarithmic solution:

$$
\begin{gathered}
\text { DEtools['formal_sol'](L, 'has logarithm?', } \mathrm{x}=1) \text {; } \\
\text { false }
\end{gathered}
$$

So L doesn't have a logarithm solution at $x=1$, and therefore $x=1$ is an apparent singularity of L.

(g) Irregular singularities of $\mathrm{M}$ are always a subset of the set of irregular singularities of L.

Example: Let us take $\mathrm{L}_{0}$ to be the Bessel square root operator $\mathrm{L}_{\check{B}_{\nu}^{2}}$ as in $(a)$ with the same change of variable parameter $f$, the same gauge parameters and the same exp-product parameter. Let us see which kind of point $x=\infty$ is for the operators $\mathrm{L}$ and $\mathrm{M}$ : 


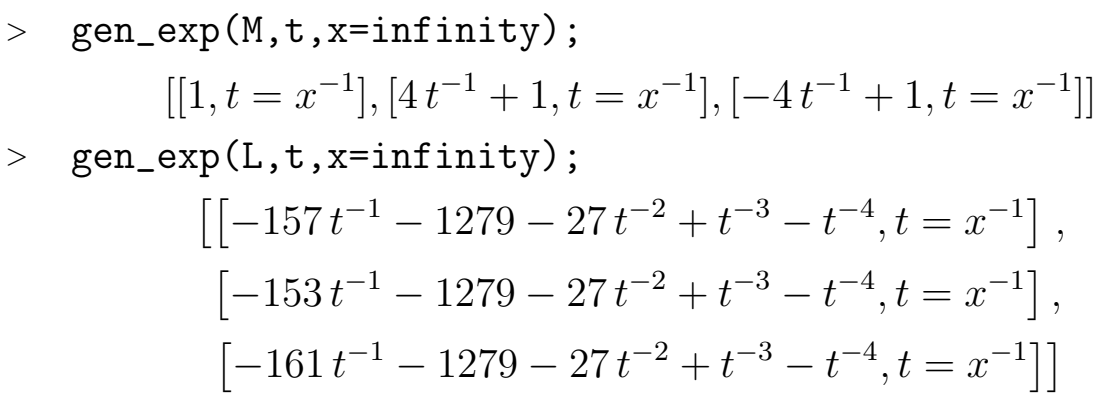

There are generalized exponents of $\mathrm{L}$ and $\mathrm{M}$ at $x=\infty$ which contain the parameter $t$, so $x=\infty$ is an irregular singularity of $\mathrm{L}$ and also of $\mathrm{M}$.

Remarks 3.5. - Looking at $(g)$, all the poles of $f$ are a subset of the set of irregular singularities of $\mathrm{L}$.

- Looking at $(a),(c)$ and $(d)$ we have that a zero of $f$ can become by exp-product and gauge transformations a regular point or an apparent singularity or regular singularity or irregular singularity of $\mathrm{L}$. Hence, it is difficult to find the zeroes of $f$.

For computing $f$, the only information retrieved from $\mathrm{L}$ that we can use is the information on the invariance under projective equivalence. The invariant we use is the difference of the exponents of $\mathrm{L}$.

\subsubsection{Exponent differences}

Definition 3.6. Let $\mathrm{L} \in \mathrm{K}[\partial]$ be a linear differential operator of order greater than one, let $p$ be any point, and $e_{1}, e_{2}$ be two generalized exponents of $\mathrm{L}$ at $p$. Then the difference $e_{2}-e_{1}$ is called an exponent difference of $\mathrm{L}$ at $p$.

If $\operatorname{deg}(\mathrm{L})=3$ there exists just three generalized exponents at each point $p: e_{1}, e_{2}$ and $e_{3}$, and we define

$$
\Delta_{1}(\mathrm{~L}, p)= \pm\left(e_{2}-e_{1}\right), \quad \Delta_{2}(\mathrm{~L}, p)= \pm\left(e_{3}-e_{1}\right), \quad \text { and } \quad \Delta_{3}(\mathrm{~L}, p)= \pm\left(e_{3}-e_{2}\right) .
$$

We define $\Delta$ modulo a factor -1 to make it well-defined because we have no ordering in the generalized exponents.

Corollary 3.7. Let $\mathrm{L} \in \mathrm{K}[\partial]$ be an irreducible third-order linear differential operator. Let $e_{1}$ and $e_{2}$ be two generalized exponents of $\mathrm{L}$ at $p$ with ramification index $n_{e_{i}}, i=1,2$. The exponent difference $e_{2}-e_{1}$ is invariant modulo $\frac{1}{n} \mathbb{Z}$ under $\longrightarrow_{E G}$, where $n \in \mathbb{N}^{*}$ is the smallest positive integer such that $\left\{\frac{1}{n_{e_{1}}} \mathbb{Z}, \frac{1}{n_{e_{2}}} \mathbb{Z}\right\} \subseteq \frac{1}{n} \mathbb{Z}$.

Proof. We just use Theorem 1.31, Lemma 2.9 and Lemma 2.10.

Remark 3.8. The exponent difference $\Delta_{i}(\mathrm{~L}, p)$ modulo $\frac{1}{n} \mathbb{Z}, i=1,2,3$, is invariant under $\left(\longrightarrow \longrightarrow_{E G}\right)$, where $n \in \mathbb{Z}$ is related to the ramification index of the generalized exponents of $\mathrm{L}$ at $p$ (see Corollary 3.7). Hence, to compute the change of variable parameter $f$, the information on the difference of the generalized exponents of $\mathrm{L}$ at its singularities will be very important for us. We will take a closed look at the part $\mathrm{L}_{0} \stackrel{f}{\longrightarrow} C \mathrm{M}$ and analyse 
$\Delta_{i}(\mathrm{~L}, p), i=1,2,3$ because, using the invariance of the exponents difference modulo $\frac{1}{n} \mathbb{Z}$ under $\longrightarrow_{E G}$, we can then apply results not only to $\mathrm{L}_{0} \stackrel{f}{\longrightarrow} C \mathrm{M}$ but also to $\mathrm{L}_{0} \stackrel{f}{\longrightarrow} C$ $\mathrm{M} \longrightarrow E G \mathrm{~L}$.

\section{Assumptions on $\mathrm{L}_{0}$}

Let $p$ be a point and $e_{i}, i=1,2,3$ the generalized exponents of $\mathrm{L}_{0}$ at $p$ with ramification index $n_{e_{i}}$. Since $e_{i} \in k\left[t_{p}^{-1 / n_{e_{i}}}\right], e_{i}$ can be put in the form

$e_{i}=\sum_{j=0}^{n_{i}} e_{j, i} t^{-j} \quad$ with $\quad b_{i} t^{n_{e_{i}}}=t_{p}, b_{i} \in k \backslash\{0\}, n_{i} \in \mathbb{N}$ and $e_{j, i} \in k, i=1,2,3$

$=\sum_{j=0}^{n_{i}}\left(e_{j, i} \sigma_{i}^{-j}\right) t_{p}^{-j / n_{e_{i}}} \quad$ with $\sigma_{i}$ solution of $X^{n_{e_{i}}}-b_{i}^{-1}=0$ with unknown $X, i=1,2,3$.

1- For $\mathrm{L}_{0} \in\left\{\mathrm{L}_{\breve{B}_{\nu}^{2}}, \mathrm{~L}_{22}, \mathrm{~L}_{12}, \mathrm{~L}_{02}, \mathrm{~L}_{11}^{2}\right\}$ and $p=0$, the generalized exponents of $\mathrm{L}_{0}$ at $p$ are

$$
e_{i}=e_{0, i}, \quad i=1,2,3 .
$$

All the other coefficients $e_{j, i}$ with $j>0$ are zero.

If we now apply Theorem 2.11 to $\mathrm{L}_{0}$ and $\mathrm{M}$ such that $\mathrm{L}_{0} \stackrel{f}{\longrightarrow}{ }_{C} \mathrm{M}$, then the generalized exponents of $\mathrm{M}$ at a zero $p$ of $f$ are $\bar{e}_{i}, i=1,2,3$ given by

$$
\bar{e}_{i}=m_{p} e_{0, i}, \quad i=1,2,3
$$

where $f=t_{p}^{m_{p}} \sum_{j=0}^{+\infty} f_{j} t_{p}^{j}, \quad$ with $f_{i} \in k$ and $f_{0} \neq 0$. Hence the ramification index of the generalized exponents of $\mathrm{M}$ at $p$ is 1 .

2- For $\mathrm{L}_{0} \in\left\{\mathrm{L}_{\breve{B}_{\nu}^{2}}, \mathrm{~L}_{22}, \mathrm{~L}_{12}, \mathrm{~L}_{02}, \mathrm{~L}_{11}^{2}\right\}$ and $p=\infty$, the generalized exponents of $\mathrm{L}_{0}$ at $p$ are

$$
e_{i}=e_{0, i}+e_{1, i} \sigma_{i}^{-1} t_{\infty}^{-1 / n_{e_{i}}}, \quad i=1,2,3 .
$$

All the others coefficients $e_{j, i}$ with $j>1$ are zero.

If we now apply Theorem 2.11 to $\mathrm{L}_{0}$ and $\mathrm{M}$ such that $\mathrm{L}_{0} \stackrel{f}{\longrightarrow} \mathrm{C}$, then the generalized exponents of $\mathrm{M}$ at a pole $p$ of $f$ are $\bar{e}_{i}, i=1,2,3$ given by

$$
\bar{e}_{i}=m_{p} e_{0, i}-e_{1, i} \sigma_{i}^{-1} \sum_{j=-m_{p}}^{-1} j \bar{f}_{j+m_{p}} t_{p}^{j / n_{e_{i}}}, \quad i=1,2,3
$$

where $\left\{\begin{aligned} f & =t_{p}^{-m_{p}} \sum_{j=0}^{+\infty} f_{j-m_{p}} t_{p}^{j}, \quad \text { with } f_{j-m_{p}} \in k \text { and } f_{-m_{p}} \neq 0, \\ \left(\sum_{j=0}^{+\infty} f_{j-m_{p}} t_{p}^{j}\right)^{1 / n_{e_{i}}} & =\sum_{j=0}^{+\infty} \bar{f}_{j} t_{p}^{j / n_{e_{i}}} \text { with } \bar{f}_{j} \in k\end{aligned}\right.$ 
Theorem 3.9. Let $\mathrm{L} \in \mathrm{K}[\partial]$ be an irreducible third-order linear differential operator and $p$ a point. Let $e_{i}, i=1,2,3$, be the generalized exponents of $\mathrm{L}$ at $p$ with ramification index $n_{e_{i}}, i=1,2,3$. Then

$$
e_{i} \in k\left[t_{p}^{-1 / n}\right] \quad \text { with } n=\max \left(n_{e_{i}}, i=1,2,3\right)
$$

Proof. Let $\mathrm{L} \in \mathrm{K}[\partial]$ be an irreducible third-order linear differential operator and $p$ a point. Let $e_{i}, i=1,2,3$, be the generalized exponents of $\mathrm{L}$ at $p$ with ramification index $n_{e_{i}}, i=1,2,3$ and $\mathrm{P}_{e_{i}}(x), i=1,2,3$ their associated minimal polynomial as in Lemma 1.34. By the definition of the generalized exponent, we have $e_{i} \in k\left[t_{p}^{-1 / n_{e_{i}}}\right], i=1,2,3$.

By Remark 1.28 we have $\mathrm{V}(\mathrm{L})=\bigoplus_{q \in \mathrm{E}} \mathrm{V}(\mathrm{L})_{q}$ with E as in Lemma 1.27. Let us rename the indices of $n_{e_{i}}, i=1,2,3$ as follows: $n_{e_{\alpha}}, n_{e_{\beta}}, n_{e_{\gamma}}$ with $\alpha, \beta, \gamma \in\{1,2,3\}$ and all different.

- If all the generalized exponents belong to different sub-modules of $\mathrm{V}(\mathrm{L})$ then

$$
\begin{gathered}
\mathrm{V}(\mathrm{L})=\bigoplus_{q \in \mathrm{E}} \mathrm{V}(\mathrm{L})_{q}=\mathrm{V}(\mathrm{L})_{e_{\alpha}} \bigoplus \mathrm{V}(\mathrm{L})_{e_{\beta}} \bigoplus \mathrm{V}(\mathrm{L})_{e_{\gamma}} \\
\Longrightarrow \operatorname{dim} \mathrm{V}(\mathrm{L})=\operatorname{dim} \mathrm{V}(\mathrm{L})_{e_{\alpha}}+\operatorname{dim} \mathrm{V}(\mathrm{L})_{e_{\beta}}+\operatorname{dim} \mathrm{V}(\mathrm{L})_{e_{\gamma}} \\
\Longrightarrow \operatorname{deg}\left(\mathrm{P}_{e_{\alpha}}(x)\right) \times n_{e_{\alpha}}+\operatorname{deg}\left(\mathrm{P}_{e_{\beta}}(x)\right) \times n_{e_{\beta}}+\operatorname{deg}\left(\mathrm{P}_{e_{\gamma}}(x)\right) \times n_{e_{\gamma}}=3 \quad \text { by } \\
\quad \operatorname{Remark} 1.33 \\
\Longrightarrow \operatorname{deg}\left(\mathrm{P}_{e_{\alpha}}(x)\right) \times n_{e_{\alpha}}=\operatorname{deg}\left(\mathrm{P}_{e_{\beta}}(x)\right) \times n_{e_{\beta}}=\operatorname{deg}\left(\mathrm{P}_{e_{\gamma}}(x)\right) \times n_{e_{\gamma}}=1 \text { since } \\
\operatorname{deg}\left(\mathrm{P}_{e_{\alpha}}(x)\right) \times n_{e_{\alpha}}, \operatorname{deg}\left(\mathrm{P}_{e_{\beta}}(x)\right) \times n_{e_{\beta}}, \operatorname{deg}\left(\mathrm{P}_{e_{\gamma}}(x)\right) \times n_{e_{\gamma}} \in \mathbb{N}^{*} \\
\Longrightarrow n_{e_{\alpha}}=n_{e_{\beta}}=n_{e_{\gamma}}=1 \text { since } \operatorname{deg}\left(\mathrm{P}_{e_{\alpha}}(x)\right), \operatorname{deg}\left(\mathrm{P}_{e_{\beta}}(x)\right), \operatorname{deg}\left(\mathrm{P}_{e_{\gamma}}(x)\right) \in \mathbb{N}^{*} .
\end{gathered}
$$

Hence $e_{\alpha}, e_{\beta}, e_{\gamma} \in k\left[t_{p}^{-1 / n}\right]$ with $n=\max \left(n_{e_{i}}, i=1,2,3\right)=1$.

- If exactly two of the generalized exponents $e_{\alpha}, e_{\beta}$ belong to the same sub-module of 
$\mathrm{V}(\mathrm{L})$, called $\mathrm{V}(\mathrm{L})_{e}$ with $e \in \mathrm{E}$ and $n_{e}$ its ramification index, then

$$
\begin{aligned}
& e_{\alpha}=e_{\beta} \text { modulo } \frac{1}{n_{e_{\beta}}} \mathbb{Z}, \quad \operatorname{dim} \mathrm{V}(\mathrm{L})_{e}=2 \text { and } \mathrm{V}(\mathrm{L})=\mathrm{V}(\mathrm{L})_{e} \bigoplus \mathrm{V}(\mathrm{L})_{e_{\gamma}} \\
& \Longrightarrow\left\{\begin{array}{l}
n_{e_{\alpha}}=n_{e_{\beta}}=n_{e}, \\
\operatorname{deg}\left(\mathrm{P}_{e}(x)\right) \times n_{e}=2 \quad \text { by Remark } 1.33, \\
\operatorname{deg}\left(\mathrm{P}_{e}(x)\right) \times n_{e}+\operatorname{deg}\left(\mathrm{P}_{e_{\gamma}}(x)\right) \times n_{e_{\gamma}}=3 \text { by Remark } 1.33
\end{array}\right. \\
& \Longrightarrow\left\{\begin{array}{l}
n_{e_{\alpha}}=n_{e_{\beta}}=n_{e} \\
\operatorname{deg}\left(\mathrm{P}_{e}(x)\right) \times n_{e}=2, \\
\operatorname{deg}\left(\mathrm{P}_{e_{\gamma}}(x)\right) \times n_{e_{\gamma}}=1
\end{array}\right. \\
& \Longrightarrow n_{e_{\alpha}}=n_{e_{\beta}}=2, n_{e} \in\{1,2\} \text { and } n_{e_{\gamma}}=1 \text { since } \operatorname{deg}\left(\mathrm{P}_{e}(x)\right) \geq 1 \text { and } \\
& \operatorname{deg}\left(\mathrm{P}_{e_{\gamma}}(x)\right) \geq 1 \\
& \Longrightarrow n_{e_{\alpha}}=n_{e_{\beta}} \in\{1,2\} \text { and } n_{e_{\gamma}}=1 \\
& \Longrightarrow\left(n_{e_{\alpha}}=n_{e_{\beta}}=n_{e_{\gamma}}=1\right) \text { or }\left(n_{e_{\alpha}}=n_{e_{\beta}}=2 \text { and } n_{e_{\gamma}}=1\right) \\
& \Longrightarrow\left\{\begin{array}{l}
n=\max \left(n_{e_{\alpha}}, n_{e_{\beta}}, n_{e_{\zeta}}\right)=1 \text { and } e_{\alpha}, e_{\beta}, e_{\gamma} \in k\left[t_{p}^{-1}\right] \\
\text { or } \\
n=\max \left(n_{e_{\alpha}}, n_{e_{\beta}}, n_{e_{\gamma}}\right)=2, e_{\alpha}, e_{\beta} \in k\left[t_{p}^{-1 / 2}\right] \text { and } e_{\gamma} \in k\left[t_{p}^{-1}\right] \subset k\left[t_{p}^{-1 / 2}\right]
\end{array}\right. \\
& \Longrightarrow e_{\alpha}, e_{\beta}, e_{\gamma} \in \mathrm{K}\left[t_{p}^{-1 / n}\right] \text { with } n=\max \left(n_{e_{\alpha}}, n_{e_{\beta}}, n_{e_{\gamma}}\right) \text {. }
\end{aligned}
$$

- If all the generalized exponents belong to the same sub-module of $\mathrm{V}(\mathrm{L})$, called $\mathrm{V}(\mathrm{L})_{e}$ with $e \in \Omega$ and $n_{e}$ its ramification index, then

$$
\begin{aligned}
& n_{e_{\alpha}}=n_{e_{\beta}}=n_{e_{\gamma}}=n_{e}, e_{\alpha}, e_{\beta}, e_{\gamma} \in \mathrm{K}\left[t_{p}^{-1 / n_{e}}\right] \text { and } n=\max \left(n_{e_{\alpha}}, n_{e_{\beta}}, n_{e_{\zeta}}\right)=n_{e} \\
\Longrightarrow & e_{\alpha}, e_{\beta}, e_{\gamma} \in \mathrm{K}\left[t_{p}^{-1 / n}\right] \text { with } n=\max \left(n_{e_{\alpha}}, n_{e_{\beta}}, n_{e_{\gamma}}\right) .
\end{aligned}
$$

Corollary 3.10. Let $\mathrm{M} \in \mathrm{K}[\partial]$ be an irreducible third-order linear differential operator such that $\mathrm{L}_{0} \stackrel{f}{\longrightarrow} C \mathrm{M}$ with $\mathrm{L}_{0} \in\left\{\mathrm{L}_{\breve{B}_{\nu}^{2}}, \mathrm{~L}_{22}, \mathrm{~L}_{12}, \mathrm{~L}_{02}, \mathrm{~L}_{11}^{2}\right\}$.

a- If $p$ is a zero of $f$ with multiplicity $m_{p} \in \mathbb{N}^{*}$ and $e_{i}, i=1,2,3$ are the generalized exponents of $\mathrm{L}_{0}$ at $x=0$ with ramification index $n_{e_{i}} \in \mathbb{N}^{*}$. Then the exponent differences of $\mathrm{M}$ at $p$ are

$$
\begin{gathered}
\Delta_{1}(\mathrm{M}, p)=m_{p}\left(e_{0,2}-e_{0,1}\right), \Delta_{2}(\mathrm{M}, p)=m_{p}\left(e_{0,3}-e_{0,1}\right) \text { and } \Delta_{3}(\mathrm{M}, p)=m_{p}\left(e_{0,3}-e_{0,2}\right) \\
\text { where }\left\{\begin{array}{r}
f=t_{p}^{m_{p}} \sum_{j=0}^{+\infty} f_{j} t_{p}^{j}, \quad \text { with } f_{i} \in k \text { and } f_{0} \neq 0, \\
e_{i}=\sum_{j=0}^{n_{i}} e_{j, i} t^{-j} \quad \text { with } b_{i} t^{n_{e_{i}}}=t_{p}, b_{i} \in k \backslash\{0\}, \\
n_{i} \in \mathbb{N} \text { and } e_{j, i} \in k, i=1,2,3 .
\end{array}\right.
\end{gathered}
$$


b- If $p$ is a pole of $f$ with multiplicity $m_{p} \in \mathbb{N}^{*}$ and $e_{i}, i=1,2,3$ are the generalized exponents of $\mathrm{L}_{0}$ at $x=\infty$ with ramification index $n_{e_{i}} \in \mathbb{N}^{*}$. Then the exponents differences of $\mathrm{M}$ at $p$ are

$$
\begin{aligned}
& \Delta_{1}(\mathrm{M}, p)=m_{p}\left(e_{0,2}-e_{0,1}\right)-\left(e_{1,2} \sigma_{2}^{-1}-e_{1,1} \sigma_{1}^{-1}\right) \sum_{j=-m_{p}}^{-1} j \bar{f}_{j+m_{p}} t_{p}^{j / n_{e_{i}}} \\
& \Delta_{2}(\mathrm{M}, p)=m_{p}\left(e_{0,3}-e_{0,1}\right)-\left(e_{1,3} \sigma_{3}^{-1}-e_{1,1} \sigma_{1}^{-1}\right) \sum_{j=-m_{p}}^{-1} j \bar{f}_{j+m_{p}} t_{p}^{j / n_{e_{i}}}, \\
& \text { and } \Delta_{3}(\mathrm{M}, p)=m_{p}\left(e_{0,3}-e_{0,2}\right)-\left(e_{1,3} \sigma_{3}^{-1}-e_{1,2} \sigma_{2}^{-1}\right) \sum_{j=-m_{p}}^{-1} j \bar{f}_{j+m_{p}} t_{p}^{j / n_{e_{i}}}
\end{aligned}
$$

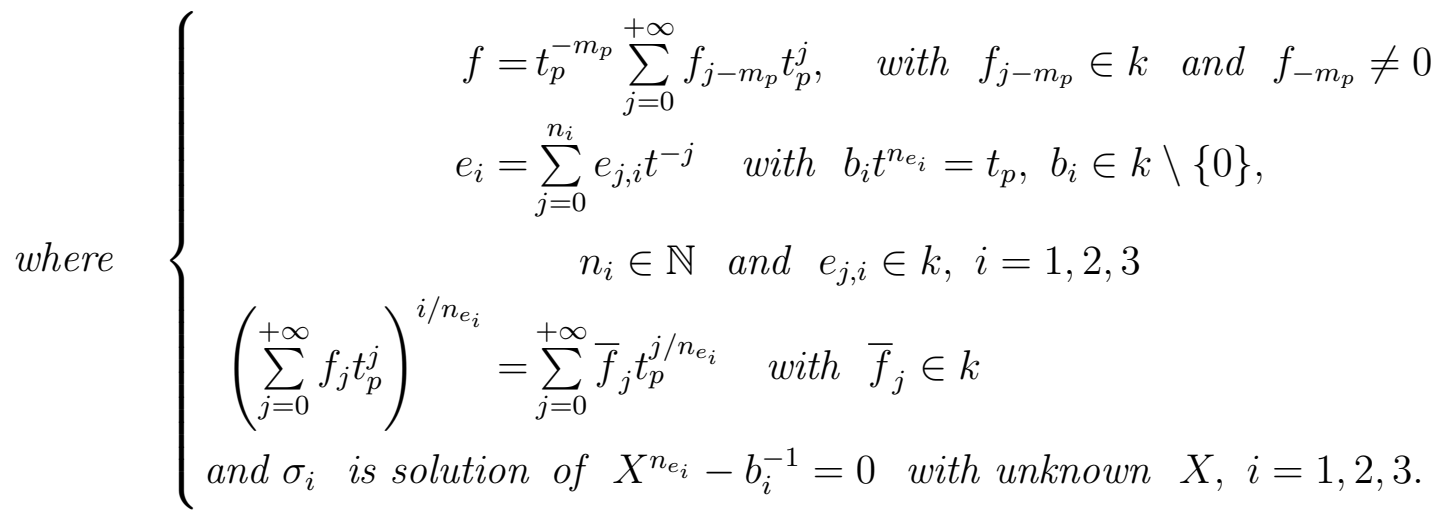

Proof. We just use the assumptions above on $\mathrm{L}_{0}$ as element of $\left\{\mathrm{L}_{\check{B}_{\nu}^{2}}, \mathrm{~L}_{22}, \mathrm{~L}_{12}, \mathrm{~L}_{02}, \mathrm{~L}_{11}^{2}\right\}$ and Theorem 3.9, and then compute the difference of the generalized exponents.

Corollary 3.11. Let $\mathrm{L}, \mathrm{M} \in \mathrm{K}[\partial]$ be irreducible third-order linear differential operators such that $\mathrm{L}_{0} \stackrel{f}{\longrightarrow} C \mathrm{M} \longrightarrow \longrightarrow_{E G} \mathrm{~L}$ with $\mathrm{L}_{0} \in\left\{\mathrm{L}_{\breve{B}_{\nu}^{2}}, \mathrm{~L}_{22}, \mathrm{~L}_{12}, \mathrm{~L}_{02}, \mathrm{~L}_{11}^{2}\right\}$. Let $p$ be a point. If $\left\{\Delta_{i}(\mathrm{~L}, p), i=1,2,3\right\} \subset k$ then the maximum of the ramification index of the generalized exponents of $\mathrm{L}$ at $p$ is 1 .

Proof. Let $e_{i}, i=1,2,3$ be the generalized exponents of $\mathrm{L}$ at $p$ with ramification index $n_{e_{i}} \in \mathbb{N}^{*}$. Let us assume that $\left\{\Delta_{i}(\mathrm{~L}, p), i=1,2,3\right\} \subset k$. Then by Corollary 3.7 about the invariance of the difference exponents modulo $\frac{1}{n} \mathbb{Z}$ ( $n$ depends on $n_{e_{i}}, i=1,2,3$ ), under the transformation $\longrightarrow_{E G}$ we have also $\left\{\Delta_{i}(\mathrm{M}, p), i=1,2,3\right\} \subset k$. Hence $p$ is a regular point or a regular singularity of $M$ since we have assumed in this thesis that $M$ will not have apparent singularities. Therefore, $p$ is a regular point of $\mathrm{M}$ or a zero of $f$ and, by our assumption part, the ramification index of the generalized exponents of $\mathrm{M}$ at $p$ is 1 . Lemma 2.9 and Lemma 2.10 show us that the ramification index doesn't change after $\longrightarrow{ }_{E G}$. That means we also have $n_{e_{i}}=1, i=1,2,3$. Hence $\max \left(n_{e_{i}}, i=1,2,3\right)=1$.

Definition 3.12. Let $\mathrm{L} \in \mathrm{K}[\partial]$ be a irreducible third-order linear differential operator. 
We define

$$
\begin{aligned}
\mathrm{S}_{\text {log }}(\mathrm{L}) & =\{p \mid \mathrm{L} \text { has logarithmic solution at } p\} \\
\mathrm{S}_{\text {reg }}(\mathrm{L}) & =\left\{p | \{ \Delta _ { i } ( \mathrm { L } , p ) , i = 1 , 2 , 3 \} \subset k \text { and } \left(\left\{\Delta_{i}(\mathrm{~L}, p), i=1,2,3\right\} \not \subset \mathbb{N}\right.\right. \\
\left.\left.\quad \text { or } p \in \mathrm{S}_{\text {log }}(\mathrm{L})\right)\right\}, & \\
\mathrm{S}_{\text {irr }}(\mathrm{L}) & =\left\{p \mid \exists i \in\{1,2,3\}, \Delta_{i}(\mathrm{~L}, p) \text { contains } t_{p}\right\}, \\
\text { and } \overline{\mathrm{S}_{\text {reg }}(\mathrm{L})} & =\left\{P_{s} \in k[x] \mid P_{s} \text { is the minimal polynomial at } s \in \mathrm{S}_{\text {reg }}(\mathrm{L}) \text { over } k\right\}, \\
& =\left\{P_{s} \in k[x] \mid P_{s} \text { is the minimal polynomial at } s \in \mathrm{S}_{\text {irr }}(\mathrm{L}) \text { over } k\right\} .
\end{aligned}
$$

We define the minimal polynomial at $\infty$ as $P_{\infty} \equiv 1$ and its degree $\operatorname{deg}\left(P_{\infty}\right) \equiv 1$.

Corollary 3.13. Let $\mathrm{L}, \mathrm{M} \in \mathrm{K}[\partial]$ be irreducible third-order linear differential operators such that $\mathrm{L}_{0} \stackrel{f}{\longrightarrow} C \mathrm{M} \longrightarrow E G$ L with $\mathrm{L}_{0} \in\left\{\mathrm{L}_{\check{B}_{\nu}^{2}}, \mathrm{~L}_{22}, \mathrm{~L}_{12}, \mathrm{~L}_{02}, \mathrm{~L}_{11}^{2}\right\}$. The following holds:

(a) $p \in \mathrm{S}_{\text {irr }}(\mathrm{L}) \Longleftrightarrow p$ is a pole of $f$, and

(b) $p \in \mathrm{S}_{\text {reg }}(\mathrm{L}) \Longrightarrow p$ is a zero of $f$.

Proof. Let $p$ be a point and $n_{p}$ be the maximum of the ramification index of L at $p$.

(a) Let us assume that $p \in \mathrm{S}_{\text {irr }}(\mathrm{L})$. Then by the definition of $\mathrm{S}_{\mathrm{irr}}(\mathrm{L})$ and the fact that $\longrightarrow{ }_{E G}$ is an equivalence relation, and also Corollary 3.7 about the invariance of the difference exponents modulo $\frac{1}{n_{p}} \mathbb{Z}$ under the transformation $\longrightarrow_{E G}$, we get

$$
\begin{aligned}
& \exists i \in\{1,2,3\}, \Delta_{i}(\mathrm{~L}, p) \text { contains } t_{p} \\
& \Longleftrightarrow \exists i \in\{1,2,3\}, \Delta_{i}(\mathrm{M}, p) \text { contains } t_{p} \\
& \Longleftrightarrow p \text { is an irregular singularity of } \mathrm{M} \\
& \Longleftrightarrow p \text { is a pole of } f .
\end{aligned}
$$

(b) Let us assume that $p \in \mathrm{S}_{\mathrm{reg}}(\mathrm{L})$. We will proceed by contradiction. Let us assume that $p$ is not a zero of $f$.

* If $p$ is a pole of $f$ then by the proof of $(a), \exists i \in\{1,2,3\}, \Delta_{i}(\mathrm{~L}, p)$ contains $t_{p}$. Hence $\left\{\Delta_{i}(\mathrm{~L}, p), i=1,2,3\right\} \not \subset k$. That is wrong by the definition of an element $p$ in $\mathrm{S}_{\mathrm{reg}}(\mathrm{L})$.

* If $p$ is not a pole of $f$ then $p$ will be a regular point of M since M doesn't have apparent singularities (regular singularities of $\mathrm{M}$ are the zeroes of $f$ and irregular singularities of $\mathrm{M}$ are the poles of $f)$. Hence $\left\{\Delta_{i}(\mathrm{M}, p), i=1,2,3\right\} \subset$ $\mathbb{N}$ (the maximum of the ramification index of $\mathrm{M}$ at $p$ is $n=1$ ) and $\mathrm{M}$ doesn't have logarithmic solution at $p$. Using Corollary 3.7 about the invariance of the difference exponents modulo $\frac{1}{n} \mathbb{Z}$ under the transformation $\longrightarrow_{E G}$ we get $\left\{\Delta_{i}(\mathrm{~L}, p), i=1,2,3\right\} \subset \mathbb{N}$. Therefore, L doesn't have logarithmic solution at $p$. That is wrong by the definition of an element $p$ in $\mathrm{S}_{\text {reg }}(\mathrm{L})$.

So we can conclude that $p$ is a zero of $f$. 


\section{Conclusion}

To find the change of variable parameter $f$ we proceed as follows:

1. We compute all the singularities of our input operator L and their associated generalized exponents.

2. We find the set $\mathrm{S}_{\mathrm{reg}}(\mathrm{L})$ and $\mathrm{S}_{\mathrm{irr}}(\mathrm{L})$.

3. For $\mathrm{S}_{\text {irr }}(\mathrm{L})$ :

(a) By Corollary 3.13 we can have all the poles of $f$.

(b) By Corollary 3.10, Corollary 3.13 and also Corollary 3.7 about the invariance of the exponent differences modulo $\frac{1}{n} \mathbb{Z}, n \in \mathbb{N}^{*}$ under $\longrightarrow_{E G}$, we can have all the multiplicity orders of all the poles of $f$.

4. For $\mathrm{S}_{\mathrm{reg}}(\mathrm{L})$ :

We have two cases

(a) when $\mathrm{S}_{\text {reg }}(\mathrm{L}) \neq \emptyset$ :

Here using Corollary 3.10, Corollary 3.13 and also Corollary 3.7 about the invariance of the exponent differences modulo $\frac{1}{n} \mathbb{Z}, n \in \mathbb{N}^{*}$ under $\longrightarrow_{E G}$,

i. If $\mathrm{S}_{\text {reg }}(\mathrm{L})$ contains all the zeroes of $f$ then we can have all the multiplicity orders of all the zeros of $f$. The only unknown will be the leading coefficient of the numerator of $f$. We will see in the next chapters how to get it. Then we will find our $f$.

ii. If $\mathrm{S}_{\mathrm{reg}}(\mathrm{L})$ gives just a part (not all) of the zeroes of $f$ then we can just have some zeroes of $f$ and their multiplicity orders. That is not enough to compute $f$. This case will be treated in the next chapters.

(b) when $\mathrm{S}_{\mathrm{reg}}(\mathrm{L})=\emptyset$ :

Here we don't know any zero of $f$ and it is difficult to find $f$. We will see what to do in the next chapters.

Now, we assume that we have found $f$ (that means also M). The next step is to find the map from $\mathrm{M}$ to $\mathrm{L}\left(\longrightarrow_{E G}\right)$.

\subsection{The second step to solve our problem}

Here we have our input third-order irreducible linear differential operator $\mathrm{L}$ and we know M (or equivalently $f$ ) which is also a third-order irreducible linear differential operator. This step is just how to find the parameters of the exp-product and gauge transformations between two third-order irreducible linear differential operators.

\subsubsection{The $p$-curvature test}

In this thesis, the $p$-curvature test (see [4], [10], [24], [30] and [42]) can increase the speed of our algorithm because it quickly eliminates most of candidates for $f$. 
Roughly speaking, the $p$-curvature of the linear differential operator $\mathrm{L}$ is a matrix that measures to what extent the solution space of $\mathrm{L}$ has dimension close to its order. One of Grothendieck's conjectures states that an irreducible linear differential operator with coefficients in $\mathbb{Q}(x)$ admits a basis of algebraic solutions over $\mathbb{Q}(x)$ if and only if its reductions modulo $p$ admit a zero $p$-curvature for almost all primes $p$.

Let $\overline{\mathbb{F}_{p}}$ denote the algebraic closure of the finite field $\mathbb{F}_{p}$ (field of characteristic $p$ ). The field of constants of $\overline{\mathbb{F}_{p}}(x)[\partial]$ is $\overline{\mathbb{F}_{p}}\left(x^{p}\right)$. We briefly introduce the idea of the $p$-curvature test here, for more details, see [10] and [30]. $\overline{\mathbb{F}_{p}}\left(x^{p}\right)$ :

The differential field $\overline{\mathbb{F}_{p}}(x)$ is a finite dimensional vector space over its field of constant

$$
\overline{\mathbb{F}_{p}}(x)=\bigoplus_{i=0}^{p-1} \overline{\mathbb{F}_{p}}\left(x^{p}\right) x^{i} .
$$

To any differential operator $\mathrm{L} \in \overline{\mathbb{F}_{p}}(x)[\partial]$, one can associate the differential module

$$
\mathcal{M}_{\mathrm{L}}:=\overline{\mathbb{F}_{p}}(x)[\partial] / \overline{\mathbb{F}_{p}}(x)[\partial] \mathrm{L}
$$

$\mathcal{M}_{\mathrm{L}}$ is a finite dimensional vector space over $\overline{\mathbb{F}_{p}}(x)$ and a basis of it is $\left(1, \partial, \ldots, \partial^{n-1}\right)$, where $n$ denotes the degree of $\mathrm{L}$ with respect to $\partial$. Multiplication by $\partial$ gives a map $\bar{\partial}$ satisfying:

$$
\bar{\partial} f m=f^{\prime} m+f \bar{\partial} m, \quad \forall m \in \mathcal{M}_{\mathrm{L}} \text { and } f \in \overline{\mathbb{F}_{p}}(x) .
$$

Definition 3.14. The p-curvature of $\mathrm{L} \in \overline{\mathbb{F}_{p}}(x)[\partial]$ is the $\overline{\mathbb{F}_{p}}(x)$-linear endomorphism of $\mathcal{M}_{\mathrm{L}}$ induced by the multiplication by the central element $\partial^{p}$. In other words, it is the $\overline{\mathbb{F}_{p}}(x)$-linear map $\bar{\partial}^{p}$ acting on the differential module $\mathcal{M}_{\mathrm{L}}$ associated to $\mathrm{L}$.

In terms of matrices we have:

Definition 3.15. The p-curvature of a differential operator $\mathrm{L}$ of order $n$ in $\overline{\mathbb{F}_{p}}(x)[\partial]$ is the $(n \times n)$ matrix with coefficients in $\overline{\mathbb{F}_{p}}(x)$, whose $(i, j)$ entry is the coefficient of $\partial^{i}$ in the remainder of the Euclidean (right) division of $\partial^{p+j}$ by $\mathrm{L}$, for $0 \leq i, j<n$.

Given $\mathrm{L} \in \overline{\mathbb{F}_{p}}(x)[\partial]$ of degree $n$, we denote by $\mathrm{A}_{p}(\mathrm{~L})$ the matrix of the $p$-curvature of $\mathrm{L}$ in the basis $\left(1, \partial, \ldots, \partial^{n-1}\right)$ and by $\mathcal{X}\left(\mathrm{A}_{p}(\mathrm{~L})\right)$ its characteristic polynomial:

$$
\mathcal{X}\left(\mathrm{A}_{p}(\mathrm{~L})\right)(X)=\operatorname{det}\left(X \cdot \mathrm{Id}_{n}-\mathrm{A}_{p}(\mathrm{~L})\right)
$$

where $\operatorname{Id}_{n}$ is the $(n \times n)$ identity matrix. Katz [24] gave the first algorithm for $\mathrm{A}_{p}(\mathrm{~L})$, based on the following matrix recurrence:

$$
\mathrm{A}_{1}=\mathrm{A}, \quad \mathrm{A}_{i+1}=\mathrm{A}_{i}^{\prime}+\mathrm{AA}_{i}
$$

where $\mathrm{A} \in \mathcal{M}\left(\overline{\mathbb{F}_{p}}(x)\right)$ is the companion matrix associated to $\mathrm{L}$. It was observed that it is slightly more efficient to replace (3.16) by the recurrence

$$
v_{i+1}=v_{i}^{\prime}+\mathrm{A} v_{i}
$$

which computes the first column $v_{i}$ of $\mathrm{A}_{i}$, by taking for $v_{1}$ the first column of $\mathrm{A}$. Then $v_{p}, \ldots, v_{p+n-1}$ are the columns of $\mathrm{A}_{p}$. This alternative requires only matrix-vector products, and thus saves a factor of $n$, but still remains quadratic in $p$. 
Lemma 3.16. Let $\mathrm{L} \in \overline{\mathbb{F}_{p}}(x)[\partial]$. If $\mathrm{L}=\mathrm{L}_{1} \mathrm{~L}_{2}$ then $\mathcal{X}\left(\mathrm{A}_{p}(\mathrm{~L})\right)=\mathcal{X}\left(\mathrm{A}_{p}\left(\mathrm{~L}_{1}\right)\right) \mathcal{X}\left(\mathrm{A}_{p}\left(\mathrm{~L}_{2}\right)\right)$ with $\operatorname{deg}\left(\mathcal{X}\left(\mathrm{A}_{p}\left(\mathrm{~L}_{i}\right)\right)\right)=\operatorname{deg}\left(\mathrm{L}_{i}\right), \quad i=1,2$.

Proof. See [42].

Theorem 3.17. Let $\mathrm{H} \subseteq \overline{\mathbb{Q}}(x)$. Let $\mathrm{L}_{1}, \mathrm{~L}_{2} \in \mathrm{H}[\partial]$ with order $n$ and $\mathrm{L}_{1} \longrightarrow \mathrm{L}_{2}$, then $\mathcal{X}\left(\mathrm{A}_{p}\left(\mathrm{~L}_{1}\right)\right)=\mathcal{X}\left(\mathrm{A}_{p}\left(\mathrm{~L}_{2}\right)\right)$ for any prime $p$.

Proof. See [42].

Remark 3.18. Let $\mathrm{K}_{p}=\overline{\mathbb{F}_{p}}(x)[y] /(F(x, y))$ be an algebraic extension of $\overline{\mathbb{F}_{p}}(x)$ of degree $n$, where $n<p$. Let $\mathcal{C}_{p}=\left\{a \in \mathrm{K}_{p} \mid a^{\prime}=0\right\}$. Then $\left[\mathrm{K}_{p}: \mathcal{C}_{p}\right]=p$ and $\mathrm{K}_{p}=\mathcal{C}_{p}+\mathcal{C}_{p} \cdot x+$ $\cdots+\mathcal{C}_{p} \cdot x^{p-1}$. Hence we can use the p-curvature for $\mathrm{K}_{p}$ in the same way in Theorem 3.17.

Proof. See [42].

Remark 3.19. $\longrightarrow_{E}$ changes $\mathcal{X}\left(\mathrm{A}_{p}(\mathrm{~L})\right)$, but not its discriminant.

To apply the $p$-curvature test between two third-order linear irreducible differential operators $\mathrm{L}_{1}$ and $\mathrm{L}_{2}$ :

1. We compute $A_{L_{1}}$ and $A_{L_{2}}$ the companion matrices associated to $L_{1}$ and $L_{2}$, respectively.

2. By the proof of Remark 3.18 in [42], we assume $p>3$ and $p$ is a prime number.

3. We compute $\mathrm{A}_{p}\left(\mathrm{~L}_{1}\right)$ and $\mathrm{A}_{p}\left(\mathrm{~L}_{2}\right)$ the matrices of the $p$-curvature of $\mathrm{L}_{1}$ and $\mathrm{L}_{2}$, respectively.

4. We compute $\mathcal{X}\left(\mathrm{A}_{p}\left(\mathrm{~L}_{1}\right)\right)$ and $\mathcal{X}\left(\mathrm{A}_{p}\left(\mathrm{~L}_{2}\right)\right)$ the characteristic polynomials associated to $\mathrm{A}_{p}\left(\mathrm{~L}_{1}\right)$ and $\mathrm{A}_{p}\left(\mathrm{~L}_{2}\right)$, respectively.

(a) If $\mathcal{X}\left(\mathrm{A}_{p}\left(\mathrm{~L}_{1}\right)\right) \neq \mathcal{X}\left(\mathrm{A}_{p}\left(\mathrm{~L}_{2}\right)\right)$, then $\mathrm{L}_{1}$ and $\mathrm{L}_{2}$ can not be related just by a gauge transformation $\left(\longrightarrow_{G}\right)$.

(b) If the discriminant $\left(\mathcal{X}\left(\mathrm{A}_{p}\left(\mathrm{~L}_{1}\right)\right)\right) \neq \operatorname{discriminant}\left(\mathcal{X}\left(\mathrm{A}_{p}\left(\mathrm{~L}_{2}\right)\right)\right)$, then $\mathrm{L}_{1}$ and $\mathrm{L}_{2}$ can not be related by an exp-product transformation $\left(\longrightarrow_{E}\right)$.

(c) If the discriminant $\left(\mathcal{X}\left(\mathrm{A}_{p}\left(\mathrm{~L}_{1}\right)\right)\right) \neq \operatorname{discriminant}\left(\mathcal{X}\left(\mathrm{A}_{p}\left(\mathrm{~L}_{2}\right)\right)\right)$, then $\mathrm{L}_{1}$ and $\mathrm{L}_{2}$ can not be related by the transformation $\longrightarrow_{E G}$.

Remark 3.20. It is true that the p-curvature test can increase the speed of our algorithm by quickly eliminating most of candidates for the change of variables parameter $f$. But since it uses one of Grothendieck's conjectures which is not yet proved, we will not use it in our implemented codes.

\subsubsection{The Exp-product Equivalence}

Definition 3.21. Two linear differential operators $\mathrm{L}_{1}$ and $\mathrm{L}_{2}$ in $k(x)[\partial]$ are exp-product equivalent if there exists $r \in k(x)$ such that

$$
\mathrm{L}_{1} \stackrel{r}{\longrightarrow} \mathrm{L}_{2}
$$

and vice versa. 
Theorem 3.22. Two linear differential operators $\mathrm{L}_{1}$ and $\mathrm{L}_{2}$ in $k(x)[\partial]$ defined as follow

$$
\begin{aligned}
& \mathrm{L}_{1}=a_{3}(x) \partial^{3}+a_{2}(x) \partial^{2}+a_{1}(x) \partial^{1}+a_{0}(x) \\
& \mathrm{L}_{2}=b_{3}(x) \partial^{3}+b_{2}(x) \partial^{2}+b_{1}(x) \partial^{1}+b_{0}(x)
\end{aligned}
$$

are related by an exp-product transformation with parameter $r, \mathrm{~L}_{1} \stackrel{r}{\longrightarrow} \mathrm{L}_{2}$, if

$$
r=\frac{a_{2}(x)-b_{2}(x)}{3}
$$

and $r$ satisfies

$$
\left\{\begin{array}{l}
a_{1}(x)-b_{1}(x)-2 r a_{2}(x)+3 r^{2}-3 r^{\prime}=0 \\
\left(r^{2}-r^{\prime}\right) a_{2}(x)-b_{0}(x)-r a_{1}(x)-r^{\prime \prime}+3 r r^{\prime}-r^{3}+a_{0}(x)=0 .
\end{array}\right.
$$

Proof. Just see the proof of Theorem 2.3.

To show that two operators $\mathrm{L}_{1}$ and $\mathrm{L}_{2}$ are exp-product equivalent, we have to

1. apply the $p$-curvature test: we see if

$$
\operatorname{discriminant}\left(\mathcal{X}\left(\mathrm{A}_{p}\left(\mathrm{~L}_{1}\right)\right)\right)=\operatorname{discriminant}\left(\mathcal{X}\left(\mathrm{A}_{p}\left(\mathrm{~L}_{2}\right)\right)\right)
$$

where $p>3$ is a prime number. (we will not use this test because of Remark 3.20).

2. compute the exp-product parameter $r$ such that $\mathrm{L}_{1} \stackrel{r}{\longrightarrow} \mathrm{L}_{2}$.

We have implemented in Maple an algorithm called ExpProdEquiv which takes as input two third-order irreducible linear differential operators $\mathrm{L}_{1}$ and $\mathrm{L}_{2}$ and returns $r$ if $\mathrm{L}_{1}$ and $\mathrm{L}_{2}$ are related just by an exp-product transformation with parameter $r$, otherwise returns 0 .

\section{Examples}

(1) Let $\mathrm{L}_{1}$ be the Bessel square root operator $\mathrm{L}_{\check{B}_{\nu}^{2}}$ with parameter $\nu=3$ and $\mathrm{L}_{2}$ the operator coming from $\mathrm{L}_{1}$ by the exp-product transformation with parameter $r=$ $\frac{(x-3)^{5}}{(x-7)^{2}}$.

$>\mathrm{L} 1:=2 * \mathrm{x}^{\wedge} 2 * \mathrm{Dx} \wedge 3+6 * \mathrm{x} * \mathrm{Dx} \wedge 2+(-2 * \mathrm{x}-16) * \mathrm{Dx}-1$;

$$
L 1:=2 x^{2} D x^{3}+6 x D x^{2}+(-2 x-16) D x-1
$$

$>r:=(x-3) \sim 5 /(x-7) \sim 2$;

$>\quad$ L2: $=$ ExpProduct $(\mathrm{L} 1, \mathrm{r})$;

$$
r:=\frac{(x-3)^{5}}{(x-7)^{2}}
$$

$$
\begin{aligned}
L 2:= & 2(x-7)^{6} x^{2} D x^{3}-6\left(x^{6}-15 x^{5}+90 x^{4}-270 x^{3}+404 x^{2}-229 x-49\right) \\
& \times(x-7)^{4} x D x^{2}+2\left(-90 x^{11}+1215 x^{10}-9720 x^{9}+51015 x^{8}-12644 x^{2}\right. \\
& -183276 x^{7}+454281 x^{6}-757351 x^{5}+784370 x^{4}-397780 x^{3}+80017 x \\
& \left.+3 x^{12}-19208\right)(x-7)^{2} D x-2 x^{17}-1890 x^{15}+24570 x^{14}-221106 x^{13}
\end{aligned}
$$




$$
\begin{aligned}
& +90 x^{16}-7276896 x^{11}+27913502 x^{10}+187904862 x^{8}-320893598 x^{7} \\
& +1458408 x^{12}-82688140 x^{9}+394295049 x^{6}-316407588 x^{5}-9452737 \\
& +119726385 x^{4}+37324028 x^{3}-65292369 x^{2}+32352936 x \\
> & \text { ExpProdEquiv(L1,L2) }
\end{aligned}
$$

$$
\left[\frac{(x-3)^{5}}{(x-7)^{2}}\right]
$$

(2) Let us change the exp-product parameter above by taking $r=(x-7)(x-12)$.

$$
\begin{aligned}
& >\quad \mathrm{r}:=(\mathrm{x}-7) *(\mathrm{x}-12) ; \\
& >\quad \mathrm{L} 2:=\operatorname{ExpProduct}(\mathrm{L} 1, \mathrm{r}) ; \\
& L 2:=2 x^{2} D x^{3}-6\left(x^{3}-19 x^{2}+84 x-1\right) x D x^{2}+\left(6 x^{6}-228 x^{5}+3174 x^{4}\right. \\
& \left.\quad-19176 x^{3}+42678 x^{2}-1010 x-16\right) D x-2 x^{8}+114 x^{7}-2670 x^{6} \\
& \quad+32888 x^{5}-224850 x^{4}+810734 x^{3}-1214174 x^{2}+42314 x+1343 \\
& >\quad \text { ExpProdEquiv(L1,L2); } \\
& \quad[(x-7)(x-12)]
\end{aligned}
$$

(3) Let us consider the case where $\mathrm{L}_{2}$ comes from $\mathrm{L}_{1}$ by a gauge transformation.

Let $\mathrm{L}_{1}$ be the same operator as in (1) and $\mathrm{L}_{2}$ the operator coming from $\mathrm{L}_{1}$ by the gauge transformation with parameters $r_{0}=x-7, r_{1}=x-9$ and $r_{2}=x-11$.

$$
\begin{aligned}
& >\quad r 0:=x-7 \text {; } \\
& r 0:=x-7 \\
& >\quad r 1:=x-9 \text {; } \\
& >\quad r 2:=x-11 \\
& r 1:=x-9 \\
& r 2:=x-11 \\
& >\text { L2: =GaugeTransf (L1, r0, r1, r2); } \\
& L 2:=2 x^{2}\left(4 x^{7}-20 x^{6}+308 x^{5}-1892 x^{4}-1535 x^{3}+17719 x^{2}-11033 x\right. \\
& \text {-12463) Dx } x^{3}-2\left(20 x^{6}-616 x^{5}+5676 x^{4}+6140 x^{3}-88595 x^{2}\right. \\
& +66198 x+87241) x D x^{2}-2\left(4 x^{7}+8 x^{6}+2474 x^{4}-38935 x^{3}\right. \\
& \left.+184 x^{5}+15413 x^{2}+158511 x-89155\right) x D x+4 x^{7}+9156 x^{4} \\
& +52 x^{6}-160 x^{5}-80677 x^{3}-11893 x^{2}+299673 x+19965 \\
& >\text { ExpProdEquiv(L1,L2); }
\end{aligned}
$$

(4) Let us consider the case where $\mathrm{L}_{2}$ comes from $\mathrm{L}_{1}$ by an exp-product transformation and a gauge transformation.

Let $\mathrm{L}_{1}$ be the same operator as in (1) and $\mathrm{L}_{2}$ the operator such that $\mathrm{L}_{1} \stackrel{r}{\longrightarrow} E$ $\mathrm{M}_{1} \stackrel{r_{0}, r_{1}, r_{2}}{\longrightarrow} \mathrm{L}_{2}$ with $r=x-1, r_{0}=0, r_{1}=x$ and $r_{2}=0$. 


$$
\begin{aligned}
& >\mathrm{r}:=\mathrm{x}-1 \\
& r:=x-1 \\
& >\quad \mathrm{M} 1:=\operatorname{ExpProduct}(\mathrm{L} 1, \mathrm{r}) \text {; } \\
& M 1:=2 x^{2} D x^{3}-6\left(x^{2}-x-1\right) D x^{2} x+\left(6 x^{4}-12 x^{3}-12 x^{2}+10 x-16\right) D x \\
& -2 x^{5}+6 x^{4}+6 x^{3}-14 x^{2}+14 x-17 \\
& >\quad r 0:=0 \text {; } \\
& r 0:=0 \\
& >\quad \mathrm{r} 1:=\mathrm{x} \\
& r 1:=x \\
& >\quad r 2:=0 \\
& r 2:=0 \\
& \text { > L2: =GaugeTransf (M1, r0, r1, r2); } \\
& L 2:=2 x^{3}\left(2 x^{5}-6 x^{4}-6 x^{3}+14 x^{2}-14 x+17\right) D x^{3}-2\left(6 x^{7}-24 x^{6}+6 x^{5}\right. \\
& \left.+48 x^{4}-90 x^{3}+93 x^{2}-37 x-34\right) D x^{2} x^{2}+2\left(-30 x^{8}+30 x^{7}+40 x^{6}\right. \\
& \left.+6 x^{9}-112 x^{5}+195 x^{4}+40 x^{3}-335 x^{2}+225 x-153\right) x D x+24 x^{10} \\
& -4 x^{11}-36 x^{9}-20 x^{8}+92 x^{7}-204 x^{6}-88 x^{5}+686 x^{4}-1624 x^{3} \\
& +1186 x^{2}-793 x+306 \\
& >\operatorname{ExpProdEquiv}(\mathrm{L} 1, \mathrm{~L} 2) \text {; }
\end{aligned}
$$

\subsubsection{The Gauge Equivalence}

Definition 3.23. Two third-order linear differential operators $\mathrm{L}_{1}$ and $\mathrm{L}_{2}$ in $k(x)[\partial]$ are gauge equivalent if there exists $r_{0}, r_{1}$ and $r_{2} \in k(x)$ such that

$$
\mathrm{L}_{1} \stackrel{r_{0}, r_{1}, r_{2}}{\longrightarrow} \mathrm{L}_{2}
$$

and vice versa.

To show that two operators $\mathrm{L}_{1}$ and $\mathrm{L}_{2}$ are gauge equivalent, we have to

1- apply the $p$-curvature test: we see if

$$
\mathcal{X}\left(\mathrm{A}_{p}\left(\mathrm{~L}_{1}\right)\right)=\mathcal{X}\left(\mathrm{A}_{p}\left(\mathrm{~L}_{2}\right)\right)
$$

where $p>3$ is a prime number. (we will not use this test because of Remark 3.20).

2- compute the gauge parameters $r_{0}, r_{1}$ and $r_{2}$ such that $\mathrm{L}_{1} \stackrel{r_{0}, r_{1}, r_{2}}{\longrightarrow} \mathrm{L}_{2}$.

There already exists in Maple an algorithm called Homomorphisms, implemented by Mark van Hoeij (see [17]), which takes as input two linear differential operators $\mathrm{L}_{1}$ and $\mathrm{L}_{2}$ of order $n$ and returns a basis where we can take an operator $\mathrm{G}$ of order $n-1$ such that $\mathrm{L}_{1}=\mathrm{L}_{2} \mathrm{G}$ or $\mathrm{L}_{2}=\mathrm{L}_{1} \mathrm{G}$, otherwise it returns an empty list. Let $n=3$ and $\mathrm{G}=$ $a_{2}(x) \partial^{2}+a_{1}(x) \partial+a_{0}(x) \partial$, we deduce the gauge parameters $r_{0}, r_{1}$ and $r_{2}$ by taking

$$
\left\{\begin{array}{l}
r_{0}=a_{0}(x), \\
r_{1}=a_{1}(x), \\
r_{2}=a_{2}(x) .
\end{array}\right.
$$




\section{Examples}

(1) Let $\mathrm{L}_{1}$ be the Bessel square root operator $\mathrm{L}_{\breve{B}_{\nu}^{2}}$ with parameter $\nu=3$ and $\mathrm{L}_{2}$ the operator coming from $\mathrm{L}_{1}$ by the gauge transformation with parameters $r_{0}=x-$ $7, r_{1}=x-9$ and $r_{2}=x-11$.

$$
\begin{aligned}
& >\mathrm{L} 1:=2 * \mathrm{x}^{\wedge} 2 * \mathrm{Dx}^{\wedge} 3+6 * \mathrm{x} * \mathrm{Dx}^{\wedge} 2+(-2 * \mathrm{x}-16) * \mathrm{Dx}-1 \text {; } \\
& L 1:=2 x^{2} D x^{3}+6 x D x^{2}+(-2 x-16) D x-1 \\
& >\mathrm{r} 0:=\mathrm{x}-7 \\
& r 0:=x-7 \\
& >\mathrm{r} 1:=\mathrm{x}-9 \\
& >\mathrm{r} 2:=\mathrm{x}-11 \\
& r 1:=x-9 \\
& r 2:=x-11 \\
& >\text { L2: =GaugeTransf (L1, r0, r1, r2); } \\
& L 2:=2 x^{2}\left(4 x^{7}-92 x^{6}+884 x^{5}-5216 x^{4}+18133 x^{3}-1505 x^{2}-132473 x\right. \\
& \text {-128623) Dx } x^{3}-2\left(92 x^{6}-1768 x^{5}+15648 x^{4}-72532 x^{3}+7525 x^{2}\right. \\
& +794838 x+900361) x D x^{2}-2\left(4 x^{7}+40 x^{5}+4454 x^{4}-57991 x^{3}\right. \\
& \left.-64 x^{6}+327005 x^{2}-322917 x-1013155\right) D x x+4 x^{7}-1576 x^{5} \\
& +28 x^{6}+14544 x^{4}-111253 x^{3}+179279 x^{2}+445665 x+19965 \\
& >\text { Homomorphisms (L1, L2); } \\
& {\left[(x-11) D x^{2}+(x-9) D x+x-7\right]}
\end{aligned}
$$

(2) Let $\mathrm{L}_{1}$ be the operator coming from the Bessel square root operator $\mathrm{L}_{\breve{B}_{\nu}^{2}}$, with parameter $\nu=3$, by the exp-product transformation with parameter $r=x-1$. Let $\mathrm{L}_{2}$ be the operator coming from $\mathrm{L}_{1}$ by the gauge transformation with parameters $r_{0}=0, r_{1}=x$ and $r_{2}=0$.

$>\quad \mathrm{LBB}:=2 * \mathrm{x}^{\wedge} 2 * \mathrm{Dx} \wedge 3+6 * \mathrm{x} * \mathrm{Dx} \bumpeq 2+(-2 * \mathrm{x}-16) * \mathrm{Dx}-1$;

$$
L B B:=2 x^{2} D x^{3}+6 x D x^{2}+(-2 x-16) D x-1
$$

$>\mathrm{r}:=\mathrm{x}-1$

$$
r:=x-1
$$

$>\quad \mathrm{L} 1:=\operatorname{ExpProduct}(\mathrm{LBB}, \mathrm{r})$;

$$
\begin{aligned}
& L 1:=2 x^{2} D x^{3}-6\left(x^{2}-x-1\right) x D x^{2}+\left(6 x^{4}-12 x^{3}-12 x^{2}+10 x-16\right) D x \\
& -2 x^{5}+6 x^{4}+6 x^{3}-14 x^{2}+14 x-17 \\
& >\mathrm{r} 0:=0 \text {; } \\
& r 0:=0 \\
& >\quad r 1:=x \\
& r 1:=x \\
& >r 2:=0 \\
& r 2:=0 \\
& >\quad \text { L2: =GaugeTransf (L1, r0, r1, r2); } \\
& L 2:=2 D x^{3} x^{3}\left(2 x^{5}-6 x^{4}-6 x^{3}+14 x^{2}-14 x+17\right)-2\left(6 x^{7}-24 x^{6}+6 x^{5}\right.
\end{aligned}
$$




$$
\begin{aligned}
& \left.+48 x^{4}-90 x^{3}+93 x^{2}-37 x-34\right) x^{2} D x^{2}+2\left(-30 x^{8}+30 x^{7}+40 x^{6}\right. \\
& \left.+6 x^{9}-112 x^{5}+195 x^{4}+40 x^{3}-335 x^{2}+225 x-153\right) D x x+24 x^{10} \\
& -36 x^{9}-20 x^{8}+92 x^{7}-204 x^{6}+686 x^{4}-1624 x^{3}+1186 x^{2}-793 x \\
& -4 x^{11}-88 x^{5}+306 \\
& >\text { Homomorphisms (L1, L2); }
\end{aligned}
$$

$$
[x D x]
$$

\subsubsection{The Projective Equivalence}

Definition 3.24. Two third-order linear differential operators $\mathrm{L}_{1}$ and $\mathrm{L}_{2}$ in $k(x)[\partial]$ are projective equivalent if there exists $r, r_{0}, r_{1}$ and $r_{2} \in k(x)$ and also $\mathrm{L}_{3} \in k(x)[\partial]$ such that

$$
\mathrm{L}_{1} \stackrel{r}{\longrightarrow} \mathrm{L}_{3}{\stackrel{r_{0}, r_{1}, r_{2}}{\longrightarrow}}_{G} \mathrm{~L}_{2}
$$

and vice versa.

We will see how to find those transformation parameters $\left(r, r_{0}, r_{1}\right.$ and $\left.r_{2}\right)$.

Definition 3.25. Let $g \in \mathrm{K}=k(x)$ and $p \in k$. The polar part of $g$ at $p$ is

$$
\begin{cases}\sum_{i=-m_{p}}^{-1} g_{i} t_{p}^{i} & \text { if } p \text { is a pole of } g \\ 0 & \text { otherwise }\end{cases}
$$

where $\sum_{i=-m_{p}}^{+\infty} g_{i} t_{p}^{i}$ is the series expansion of $g$ at $p, g_{i} \in k$ and $m_{p} \in \mathbb{N} \backslash\{0\}$. We denote it by $\operatorname{Polar}(g, p) . m_{p}$ is called the multiplicity order of $p$ as a pole of $g$.

Definition 3.26. Let $g \in \mathrm{K}=k(x)$. Then $g$ can be defined in terms of its poles as follows:

$$
g=c+\sum_{p \in \mathbb{P}} \operatorname{Polar}(g, p)
$$

where $c \in k$ and $\mathbb{P}=\mathbb{P}_{0} \cup\{\infty\}$ with $\mathbb{P}_{0}$ the set of poles of $g$. We call $\sum_{p \in \mathbb{P}} \operatorname{Polar}(g, p)$ the polar part of $g$.

Theorem 3.27. Let $g \in \mathrm{K}=k(x)$. Then the constant term $c$ in the expansion of $g$ as in Definition 3.26 is the same as the constant in the series expansion of $g$ at $\infty$.

Proof. Let us consider the series expansion of $g$ at $\infty$ given by $\sum_{i=-m_{p}}^{+\infty} g_{i} t_{\infty}^{i}$. Then

$$
g=\operatorname{Polar}(g, \infty)+g_{0}+\sum_{i=1}^{+\infty} g_{i} t_{\infty}^{i} .
$$

By using the expansion of $g$ as in Definition 3.26 we have

$$
g=\operatorname{Polar}(g, \infty)+c+\sum_{p \in \mathbb{P} \backslash\{\infty\}} \operatorname{Polar}(g, p) .
$$


Since $g$ can be also be brought in the form $\frac{h_{1}}{h_{2}}+a+\operatorname{Polar}(g, \infty)$ with $h_{1}, h_{2} \in k[x], a \in k$ and $\operatorname{deg}\left(h_{1}\right)<\operatorname{deg}\left(h_{2}\right)$ or $h_{1}=0$, we conclude by (3.19) and (3.20) that

$$
\sum_{p \in \mathbb{P} \backslash\{\infty\}} \operatorname{Polar}(g, p)=\frac{h_{1}}{h_{2}}=\sum_{i=1}^{+\infty} g_{i} t_{\infty}^{i} \quad \text { and } \quad c=a=g_{0} .
$$

Theorem 3.28. Let $\mathrm{L}_{1}, \mathrm{~L}_{2} \in k(x)[\partial]$ be two irreducible third-order linear differential operators and $r \in k(x)$ the exp-product parameter such that $\mathrm{L}_{1} \stackrel{r}{\longrightarrow} \mathrm{L}_{2}$. Let $p$ be a pole of $r$ with multiplicity $m_{p}$ such that $m_{p} \geq 2$ if $p \neq \infty$. If we assume that $\mathrm{L}_{1}$ is not the image of an exp-product transformation with rational function $-r+a_{p} t_{p}^{-1}$ with $a_{p} \in k$, then $p$ is an irregular singularity of $\mathrm{L}_{2}$.

Proof. Let $p$ be a pole of $r$ of order $m_{p}$ and $e$ a generalized exponent of $\mathrm{L}_{1}$ at $p$. Then by Lemma 2.9 a generalized exponent of $\mathrm{L}_{2}$ at $p$ is

$$
\bar{e}=\left\{\begin{array}{l}
e+\sum_{i=-m_{p}}^{-1} r_{i} t_{p}^{i+1} \text { if } p \neq \infty \\
e-\sum_{i=-m_{\infty}}^{1} r_{i} t_{\infty}^{i-1} \text { otherwise }
\end{array}\right.
$$

where $\sum_{i=-m_{p}}^{+\infty} r_{i} t_{p}^{i}$ is the series expansion of $r$ at $p$. Since $\mathrm{L}_{1}$ doesn't come from an operator by the exp-product transformation of parameter $-r+a_{p} t_{p}^{-1}$ with $a_{p} \in k$, and $m_{p} \geq 2$ if $p \neq \infty$, e cannot be written in the form

$$
e=\left\{\begin{array}{l}
b-\sum_{i=-m_{p}}^{-2} r_{i} t_{p}^{i+1} \text { if } p \neq \infty \\
b+\sum_{i=-m_{\infty}}^{0} r_{i} t_{\infty}^{i-1} \text { otherwise }
\end{array}\right.
$$

with $b \in k$. Hence, we will always have in $\bar{e}$ the parameter $t_{p}$. So $\bar{e} \notin k$ and we conclude that $p$ is an irregular singularity of $\mathrm{L}_{2}$.

Theorem 3.29. Let $\mathrm{L}_{1}, \mathrm{~L}_{2} \in k(x)[\partial]$ be two irreducible third-order linear differential operators and $r \in k(x)$ the exp-product parameter such that $\mathrm{L}_{1} \stackrel{r}{\longrightarrow} \mathrm{L}_{2}$. Let $\mathbb{P}_{0}$ be the set of poles of $r$ and for all $p \in \mathbb{P}_{0}, m_{p}$ its multiplicity order as a pole of $r$. For all $p \in k$, let $\bar{e}_{p}^{i}, i \in\{1,2,3\}$, be the generalized exponents of $\mathrm{L}_{2}$ at $p$. If we assume that $\mathrm{L}_{1}$ is not the image of an exp-product transformation with rational function $-r+a_{p} t_{p}^{-1}$ with $a_{p} \in k$ and $p \in \mathbb{P}_{0}$ such that $m_{p} \geq 2$ if $p \neq \infty$, then

$$
\sum_{p \in \mathbb{S}} \operatorname{Polar}(r, p)=\sum_{p \in \mathbb{P}} \operatorname{Polar}(r, p)-\sum_{p \in \mathbb{P}_{0}^{1} \backslash \mathbb{S}} \operatorname{Polar}(r, p)
$$

where $\mathbb{S}$ is the set of all non-apparent singularities of $\mathrm{L}_{2}, \mathbb{P}=\mathbb{P}_{0} \cup\{\infty\}$ and $\mathbb{P}_{0}^{1}=\left\{p \in \mathbb{P}_{0} \mid\left\{\bar{e}_{p}^{1}, \bar{e}_{p}^{2}, \bar{e}_{p}^{3}\right\} \subseteq \mathbb{Z}\right\}$ 
Proof. For all $p \in k$ let $\bar{e}_{p}^{i}, i \in\{1,2,3\}$, be the generalized exponents of $\mathrm{L}_{2}$ at $p$. Let us define also the following sets:

$$
\begin{aligned}
& \mathbb{P}_{0}^{2}=\left\{p \in \mathbb{P}_{0} \mid\left\{\bar{e}_{p}^{1}, \bar{e}_{p}^{2}, \bar{e}_{p}^{3}\right\} \subseteq k \text { and } \exists i \in\{1,2,3\}, \bar{e}_{p}^{i} \notin \mathbb{Z}\right\} \\
& \mathbb{P}_{0}^{3}=\left\{p \in \mathbb{P}_{0} \mid m_{p} \geq 2\right\}
\end{aligned}
$$

We have $\mathbb{P}_{0}=\mathbb{P}_{0}^{1} \cup \mathbb{P}_{0}^{2} \cup \mathbb{P}_{0}^{3}$ and for all $p \in \mathbb{P}_{0}^{2}$ (resp. $p \in \mathbb{P}_{0}^{3}$ ), $p$ is a regular singularity of $\mathrm{L}_{2}$ (resp. $p$ is an irregular singularity of $\mathrm{L}_{2}$ using Theorem 3.28). Hence, $\mathbb{P}_{0}^{2} \cup \mathbb{P}_{0}^{3} \subseteq \mathbb{S}$. By Definition 3.25, the polar part of $r$ at $p \notin \mathbb{P}_{0}$ is zero since $p$ is not a pole of $r$. Therefore,

$$
\begin{aligned}
& \sum_{p \in \mathbb{S}} \operatorname{Polar}(r, p)=\sum_{p \in \mathbb{S} \cap \mathbb{P}_{0}} \operatorname{Polar}(r, p)=\sum_{p \in \mathbb{P}_{0}^{2} \cup \mathbb{P}_{0}^{3}} \operatorname{Polar}(r, p)+\sum_{p \in \mathbb{P}_{0}^{1} \cap \mathbb{S}} \operatorname{Polar}(r, p) \\
& =\sum_{p \in \mathbb{P}_{0}} \operatorname{Polar}(r, p)-\sum_{p \in \mathbb{P}_{0}^{1} \backslash \mathbb{S}} \operatorname{Polar}(r, p) \text {. }
\end{aligned}
$$

Using again Definition 3.25, Polar $(r, \infty)=0$ if $\infty \notin \mathbb{P}_{0}$ and then

$$
\begin{aligned}
\sum_{p \in \mathbb{S}} \operatorname{Polar}(r, p) & =\sum_{p \in \mathbb{P}_{0} \cup\{\infty\}} \operatorname{Polar}(r, p)-\sum_{p \in \mathbb{P}_{0}^{1} \backslash \mathbb{S}} \operatorname{Polar}(r, p) \\
& =\sum_{p \in \mathbb{P}} \operatorname{Polar}(r, p)-\sum_{p \in \mathbb{P}_{0}^{1} \backslash \mathbb{S}} \operatorname{Polar}(r, p) .
\end{aligned}
$$

Theorem 3.30. Let $\mathrm{L}_{1}, \mathrm{~L}_{2}, \mathrm{~L}_{3} \in k(x)[\partial]$ be three irreducible third-order linear differential operators, $r, r_{0}, r_{1}, r_{2} \in k(x)$ such that $\mathrm{L}_{1} \stackrel{r}{\longrightarrow}{ }_{E} \mathrm{~L}_{2} \stackrel{r_{0}, r_{1}, r_{2}}{\longrightarrow}{ }_{G} \mathrm{~L}_{3}$. Let $\mathbb{P}_{0}$ be the set of poles of $r$ and for all $p \in \mathbb{P}_{0}, m_{p}$ its multiplicity order as a pole of $r$. For all $p \in k$, let $\bar{e}_{p}^{i}, \quad i \in\{1,2,3\}$, be the generalized exponents of $\mathrm{L}_{3}$ at $p$. If we assume that $\mathrm{L}_{1}$ is not the image of an exp-product transformation with rational function $-r+a_{p} t_{p}^{-1}$ with $a_{p} \in k$ and $p \in \mathbb{P}_{0}$ such that $m_{p} \geq 2$ if $p \neq \infty$, then

$$
\sum_{p \in \mathbb{S}} \operatorname{Polar}(r, p)=\sum_{p \in \mathbb{P}} \operatorname{Polar}(r, p)-\sum_{p \in \mathbb{P}_{0}^{1} \backslash \mathbb{S}} \operatorname{Polar}(r, p)
$$

where $\mathbb{S}$ is the set of all non-apparent singularities of $\mathrm{L}_{3}, \mathbb{P}=\mathbb{P}_{0} \cup\{\infty\}$ and $\mathbb{P}_{0}^{1}=\left\{p \in \mathbb{P}_{0} \mid\left\{\bar{e}_{p}^{1}, \bar{e}_{p}^{2}, \bar{e}_{p}^{3}\right\} \subseteq \mathbb{Z}\right\}$.

Proof. We just use Theorem 3.28 and the fact that, by gauge transformation, the generalized exponents vary by a constant. So an irregular singularity of $\mathrm{L}_{2}$ remains an irregular singularity of $\mathrm{L}_{3}$. The rest follows as in the proof of Theorem 3.29.

Now we will show how to find the exp-product parameter in the projective equivalence.

Theorem 3.31. Let $\mathrm{L}_{1}, \mathrm{~L}_{2} \in k(x)[\partial]$ be two irreducible third-order linear differential operators such that $\mathrm{L}_{1} \longrightarrow_{E G} \mathrm{~L}_{2}$ and $r$ the parameter of the exp-product transformation. Let $\mathbb{S}$ be the set of all non-apparent singularities of $\mathrm{L}_{2}$ and $\mathbb{P}_{0}$ the set of all the poles of $r$. For $p \in \mathbb{P}_{0} \cup \mathbb{S}$, let us set

$$
e_{p}^{i}=e_{p}^{i}\left(\mathrm{~L}_{2}\right)-e_{p}^{i}\left(\mathrm{~L}_{1}\right), \quad i=1,2,3
$$


where $e_{p}^{i}\left(\mathrm{~L}_{j}\right)$ is the $i^{\text {th }}$ generalized exponent of $\mathrm{L}_{j}$ at $p, j \in\{1,2\}$, and $r$ has series representation

$$
r=\sum_{i=-m_{p}}^{+\infty} r_{p, i} t_{p}^{i}, \quad m_{p} \in \mathbb{N} \quad \text { with } r_{p, i} \in k \text { and } r_{p,-m_{p}} \neq 0 .
$$

If we assume that

(a)- $\mathbb{P}_{0}^{11}=\left\{p \in \mathbb{P}_{0} \mid\left\{e_{p}^{1}\left(\mathrm{~L}_{2}\right), e_{p}^{2}\left(\mathrm{~L}_{2}\right), e_{p}^{3}\left(\mathrm{~L}_{2}\right)\right\} \subseteq \mathbb{Z}\right.$ and $\left.r_{p,-1} \notin \mathbb{Z}\right\}=\emptyset$

(b)- $\mathrm{L}_{1}$ is not the image of an exp-product transformation with rational function $-r+$ $a_{p} t_{p}^{-1}$ with $a_{p} \in k$ and $p \in \mathbb{P}_{0}$ such that $m_{p} \geq 2$ if $p \neq \infty$,

then

$$
\sum_{p \in \mathbb{S} \backslash\{\infty\}} \frac{e_{p}^{i}}{t_{p}}-t_{\infty} \cdot \overline{e_{\infty}^{i}}=r+\sum_{p \in \mathbb{S} \backslash\{\infty\}} \frac{b_{p}}{n_{p}} t_{p}^{-1}-\sum_{p \in \mathbb{P}_{0}^{12} \backslash(\mathbb{S} \cup\{\infty\})} r_{p,-1} t_{p}^{-1}
$$

where $\overline{e_{\infty}^{i}}=e_{\infty}^{i}-\operatorname{const}\left(e_{\infty}^{i}\right)$ with const $\left(e_{\infty}^{i}\right)$ the constant term of $e_{\infty}^{i}, b_{p} \in \mathbb{Z}$, $n_{p}=\max \left\{n_{e_{p}^{i}\left(\mathrm{~L}_{2}\right)}, i=1,2,3\right\}$ with $n_{e_{p}^{i}\left(\mathrm{~L}_{2}\right)}$ the ramification index of $e_{p}^{i}\left(\mathrm{~L}_{2}\right)$, and $\mathbb{P}_{0}^{12}=\left\{p \in \mathbb{P}_{0} \mid\left\{e_{p}^{1}\left(\mathrm{~L}_{2}\right), e_{p}^{2}\left(\mathrm{~L}_{2}\right), e_{p}^{3}\left(\mathrm{~L}_{2}\right), r_{p,-1}\right\} \subseteq \mathbb{Z}\right\}$

Proof. Let $p \in \mathbb{S}$ and $i \in\{1,2,3\}$ :

* If $p \neq \infty$ then by Lemma 2.9 and Lemma 2.10

$$
e_{p}^{i}\left(\mathrm{~L}_{2}\right)= \begin{cases}e_{p}^{i}\left(\mathrm{~L}_{1}\right)+\sum_{j=-m_{p}}^{-1} r_{p, j} t_{p}^{j+1}+\frac{b_{p}}{n_{p}}, \quad b_{p} \in \mathbb{Z} & \text { if } p \text { is a pole of } r, \\ e_{p}^{i}\left(\mathrm{~L}_{1}\right)+\frac{b_{p}}{n_{p}}, \quad b_{p} \in \mathbb{Z} & \text { otherwise }\end{cases}
$$

Since $e_{p}^{i}=e_{p}^{i}\left(\mathrm{~L}_{2}\right)-e_{p}^{i}\left(\mathrm{~L}_{1}\right)$, we have

$$
\begin{aligned}
\frac{e_{p}^{i}}{t_{p}} & = \begin{cases}\sum_{j=-m_{p}}^{-1} r_{p, j} t_{p}^{j}+\frac{b_{p}}{n_{p}} t_{p}^{-1}, \quad b_{p} \in \mathbb{Z} & \text { if } p \text { is a pole of } r \\
\frac{b_{p}}{n_{p}}, \quad b_{p} \in \mathbb{Z} & \text { otherwise }\end{cases} \\
& =\operatorname{Polar}(r, p)+\frac{b_{p}}{n_{p}} t_{p}^{-1}, \quad b_{p} \in \mathbb{Z} .
\end{aligned}
$$

* If $p=\infty$ then by Lemma 2.9 and Lemma 2.10

$$
e_{\infty}^{i}\left(\mathrm{~L}_{2}\right)= \begin{cases}e_{\infty}^{i}\left(\mathrm{~L}_{1}\right)-\sum_{j=m_{\infty}}^{1} r_{\infty, j} t_{\infty}^{j-1}+\frac{b_{\infty}}{n_{\infty}}, \quad b_{\infty} \in \mathbb{Z} & \text { if } \infty \text { is a pole of } r \\ e_{p}^{i}\left(\mathrm{~L}_{1}\right)-r_{\infty, 0} t_{\infty}^{-1}-r_{\infty, 1}+\frac{b_{\infty}}{n_{\infty}}, b_{\infty} \in \mathbb{Z} & \text { otherwise }\end{cases}
$$




$$
\begin{aligned}
\text { Since } e_{\infty}^{i}=e_{\infty}^{i}\left(\mathrm{~L}_{2}\right) & -e_{\infty}^{i}\left(\mathrm{~L}_{1}\right) \text { then } \\
e_{\infty}^{i} & =\left\{\begin{array}{l}
-\sum_{j=m_{\infty}}^{1} r_{\infty, j} t_{\infty}^{j-1}+\frac{b_{\infty}}{n_{\infty}}, b_{\infty} \in \mathbb{Z} \text { if } \infty \text { is a pole of } r, \\
-r_{\infty, 0} t_{\infty}^{-1}-r_{\infty, 1}+\frac{b_{\infty}}{n_{\infty}}, b_{\infty} \in \mathbb{Z} \text { otherwise }
\end{array}\right. \\
\Longrightarrow \overline{e_{\infty}^{i}} & =\left\{\begin{array}{l}
e_{\infty}^{i}-\left(-r_{\infty, 1}+\frac{b_{\infty}}{n_{\infty}}\right), b_{\infty} \in \mathbb{Z} \text { if } \infty \text { is a pole of } r \\
e_{\infty}^{i}-\left(-r_{\infty, 1}+\frac{b_{\infty}}{n_{\infty}}\right), b_{\infty} \in \mathbb{Z} \text { otherwise }
\end{array}\right. \\
\Longrightarrow t_{\infty} \cdot \overline{e_{\infty}^{i}} & =\left\{\begin{array}{l}
-\sum_{j=m_{\infty}}^{0} r_{\infty, j} t_{\infty}^{j} \text { if } \infty \text { is a pole of } r, \\
-r_{\infty, 0} \text { otherwise } \\
-r_{\infty, 0}-\sum_{j=m_{\infty}}^{-1} r_{\infty, j} t_{\infty}^{j} \text { if } \infty \text { is a pole of } r, \\
-r_{\infty, 0} \text { otherwise } \\
-\left(r_{\infty, 0}+\sum_{j=m_{\infty}}^{-1} r_{\infty, j} t_{\infty}^{j}\right) \text { if } \infty \text { is a pole of } r . \\
-r_{\infty, 0} \text { otherwise }
\end{array}\right. \\
& =-\left(r_{\infty, 0}+\operatorname{Polar}(r, \infty)\right) .
\end{aligned}
$$

Let $\mathbb{P}_{0}^{1}=\left\{p \in \mathbb{P}_{0} \mid\left\{e_{p}^{1}\left(\mathrm{~L}_{2}\right), e_{p}^{2}\left(\mathrm{~L}_{2}\right), e_{p}^{3}\left(\mathrm{~L}_{2}\right)\right\} \subseteq \mathbb{Z}\right\}$. Using (3.24) and (3.26), we get

$$
\begin{aligned}
\sum_{p \in \mathbb{S} \backslash\{\infty\}} \frac{e_{p}^{i}}{t_{p}}-t_{\infty} \cdot \overline{e_{\infty}^{i}}= & r_{\infty, 0}+\operatorname{Polar}(r, \infty)+\sum_{p \in \mathbb{S} \backslash\{\infty\}} \operatorname{Polar}(r, p)+\sum_{p \in \mathbb{S} \backslash\{\infty\}} \frac{b_{p}}{n_{p}} t_{p}^{-1} \\
= & r_{\infty, 0}+\operatorname{Polar}(r, \infty)+\sum_{p \in \mathbb{P} \backslash\{\infty\}} \operatorname{Polar}(r, p)-\sum_{p \in \mathbb{P}_{0}^{1} \backslash(\mathbb{S} \cup\{\infty\})} \operatorname{Polar}(r, p) \\
& +\sum_{p \in \mathbb{S} \backslash\{\infty\}} \frac{b_{p}}{n_{p}} t_{p}^{-1} \text { by Theorem } 3.30 \\
= & r_{\infty, 0}+\sum_{p \in \mathbb{P}} \operatorname{Polar}(r, p)+\sum_{p \in \mathbb{S} \backslash\{\infty\}} \frac{b_{p}}{n_{p}} t_{p}^{-1}-\sum_{p \in \mathbb{P}_{0}^{1} \backslash(\mathbb{S} \cup\{\infty\})} \operatorname{Polar}(r, p) .
\end{aligned}
$$

Using the fact that $r_{\infty, 0}$ is the constant term in the series expansion of $r$ at $\infty$ and also Definition 3.26 and Theorem 3.27 we have

$$
\sum_{p \in \mathbb{S} \backslash\{\infty\}} \frac{e_{p}^{i}}{t_{p}}-t_{\infty} \cdot \overline{e_{\infty}^{i}}=r+\sum_{p \in \mathbb{S} \backslash\{\infty\}} \frac{b_{p}}{n_{p}} t_{p}^{-1}-\sum_{p \in \mathbb{P}_{0}^{1} \backslash(\mathbb{S} \cup\{\infty\})} \operatorname{Polar}(r, p) .
$$

Since $\mathbb{P}_{0}^{1}=\mathbb{P}_{0}^{11} \cup \mathbb{P}_{0}^{12}$, and by assumption $\mathbb{P}_{0}^{11}=\emptyset$, we have $\mathbb{P}_{0}^{1}=\mathbb{P}_{0}^{12}$. For $p \in \mathbb{P}_{0}^{1}$, if $m_{p} \geq 2$ then using Theorem 3.28, $p$ will be an irregular singularity of $\mathrm{L}_{2}$ and that is not true by the definition of $\mathbb{P}_{0}^{1}$. Therefore, $m_{p}=1$ and then

$$
\sum_{p \in \mathbb{P}_{0}^{1} \backslash(\mathbb{S} \cup\{\infty\})} \operatorname{Polar}(r, p)=\sum_{p \in \mathbb{P}_{0}^{1} \backslash(\mathbb{S} \cup\{\infty\})} r_{p,-1} t_{p}^{-1}=\sum_{p \in \mathbb{P}_{0}^{12} \backslash(\mathbb{S} \cup\{\infty\})} r_{p,-1} t_{p}^{-1} .
$$


Hence,

$$
\sum_{p \in \mathbb{S} \backslash\{\infty\}} \frac{e_{p}^{i}}{t_{p}}-t_{\infty} \cdot \overline{e_{\infty}^{i}}=r+\sum_{p \in \mathbb{S} \backslash\{\infty\}} \frac{b_{p}}{n_{p}} t_{p}^{-1}-\sum_{p \in \mathbb{P}_{0}^{12} \backslash(\mathbb{S} \cup\{\infty\})} r_{p,-1} t_{p}^{-1}
$$

Lemma 3.32. Let us consider the hypothesis and notations of Theorem 3.31, and assume that all the conditions in Theorem 3.31 are also satisfied. Then the parameter $r$ of the exp-product transformation is given by

$$
r=\sum_{p \in \mathbb{S} \backslash\{\infty\}} \frac{e_{p}^{i}}{t_{p}}-t_{\infty} \cdot \overline{e_{\infty}^{i}}+\sum_{p \in \mathbb{S} \backslash\{\infty\}} \frac{c_{p}}{n_{p}} t_{p}^{-1}
$$

with $c_{p} \in \mathbb{Z}$ and $\left|c_{p}\right|<n_{p}$.

Proof. By Theorem 3.31 we have

$$
\begin{aligned}
r & =\sum_{p \in \mathbb{S} \backslash\{\infty\}} \frac{e_{p}^{i}}{t_{p}}-t_{\infty} \cdot \overline{e_{\infty}^{i}}-\sum_{p \in \mathbb{S} \backslash\{\infty\}} \frac{b_{p}}{n_{p}} t_{p}^{-1}+\sum_{p \in \mathbb{P}_{0}^{12} \backslash(\mathbb{S} \cup\{\infty\})} r_{p,-1} t_{p}^{-1} \\
& =\sum_{p \in \mathbb{S} \backslash\{\infty\}} \frac{e_{p}^{i}}{t_{p}}-t_{\infty} \cdot \overline{e_{\infty}^{i}}+\sum_{p \in \mathbb{S} \backslash\{\infty\}} \frac{c_{p}}{n_{p}} t_{p}^{-1}+\sum_{p \in \mathbb{S} \backslash\{\infty\}} \frac{d_{p}}{n_{p}} t_{p}^{-1}+\sum_{p \in \mathbb{P}_{0}^{12} \backslash(\mathbb{S} \cup\{\infty\})} r_{p,-1} t_{p}^{-1}
\end{aligned}
$$

where $-b_{p}=c_{p}+d_{p}$ with $c_{p}, d_{p} \in \mathbb{Z}$ and $\left|c_{p}\right|<n_{p}$. Let $y$ be a solution of $\mathrm{L}_{1}$. Since we are searching solutions $\bar{y}$ of $\mathrm{L}_{2}$ in the form

$$
\bar{y}=\exp \left(\int r d x\right)\left(r_{0} y+r_{1} y^{\prime}+r_{2} y^{\prime \prime}\right)
$$

the term $\sum_{p \in \mathbb{S} \backslash\{\infty\}} \frac{d_{p}}{n_{p}} t_{p}^{-1}+\sum_{p \in \mathbb{P}_{0}^{12} \backslash(\mathbb{S} \cup\{\infty\})} r_{p,-1} t_{p}^{-1}$ in the expression of $r$ will be transformed as follows:

$$
\begin{aligned}
& \exp \left(\int\left(\sum_{p \in \mathbb{S} \backslash\{\infty\}} \frac{d_{p}}{n_{p}} t_{p}^{-1}+\sum_{p \in \mathbb{P}_{0}^{12} \backslash(\mathbb{S} \cup\{\infty\})} r_{p,-1} t_{p}^{-1}\right) d x\right)\left(r_{0} y+r_{1} y^{\prime}+r_{2} y^{\prime \prime}\right) \\
= & x^{p \sum^{p \backslash\{\infty\}\}}{ }^{d_{p}}}+\sum_{p \in \mathbb{P}_{0}^{12} \backslash(\mathbb{S} \cup\{\infty\})}{ }^{r_{p,-1}}\left(r_{0} y+r_{1} y^{\prime}+r_{2} y^{\prime \prime}\right) \\
= & \overline{r_{0}} y+\overline{r_{1}} y^{\prime}+\bar{r}_{2} y^{\prime \prime}
\end{aligned}
$$

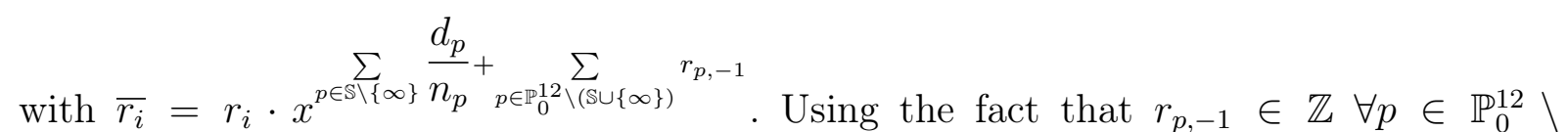
$(\mathbb{S} \cup\{\infty\})$, and also $d_{p} \in \mathbb{Z} \forall p \in \mathbb{S} \backslash\{\infty\}$, we will have $\overline{r_{0}}, \overline{r_{1}}, \overline{r_{2}} \in k(x)$. Therefore, we can take the exp-product parameter $r$ as

$$
r=\sum_{p \in \mathbb{S} \backslash\{\infty\}} \frac{e_{p}^{i}}{t_{p}}-t_{\infty} \cdot \overline{e_{\infty}^{i}}+\sum_{p \in \mathbb{S} \backslash\{\infty\}} \frac{c_{p}}{n_{p}} t_{p}^{-1}
$$

and that will just change the gauge transformation parameters $r_{1}, r_{2}$ and $r_{3}$.

We know now how to find the exp-product parameter in the projective equivalence. What remains for us is to show how to find the gauge parameters in the projective equivalence. 
Definition 3.33. Let $\mathrm{L}_{1}, \mathrm{~L}_{2} \in k(x)[\partial]$. The symmetric product of $\mathrm{L}_{1}$ and $\mathrm{L}_{2}$, denoted $\mathrm{L}_{1} \mathrm{~S} \mathrm{~L}_{2}$, is the smallest order monic operator $\mathrm{L} \in k(x)[\partial]$ for which $y_{1} y_{2} \in \mathrm{V}(\mathrm{L})$ for every $y_{1} \in \mathrm{V}\left(\mathrm{L}_{1}\right)$ and $y_{2} \in \mathrm{V}\left(\mathrm{L}_{2}\right)$. The operator $\mathrm{L}^{\circledR m}$ is called the $m^{\text {th }}$ symmetric power of $\mathrm{L}$, it is the symmetric product of $m$ copies of $\mathrm{L}$.

More details about the symmetric product can be found in [20], [19] and [26].

Definition 3.34. Let $\mathrm{L} \in k(x)[\partial]$. A hyperexponential solution of $\mathrm{L}$ over $k(x)$ is a nonzero $y \in \mathrm{V}(\mathrm{L})$ for which $y^{\prime} / y \in k(x)$. This corresponds to a first order right-hand $\partial-r$ of $\mathrm{L}$ where $r=y^{\prime} / y$. We will denote a non-zero solution $y$ of $\partial-r$ as $\exp \left(\int r d x\right)$.

Remark 3.35. By assuming that we know the exp-product parameter $r$, Definition 3.33 and Definition 3.34 will help us to find the middle operator $\mathrm{L}_{2}$ in the transformation $\mathrm{L}_{1} \stackrel{r}{\longrightarrow}_{E} \mathrm{~L}_{2} \longrightarrow_{G} \mathrm{~L}_{3}$. It is given by

$$
\mathrm{L}_{2}=\mathrm{L}_{1} \mathrm{~S}(\partial-r)
$$

Hence, we just have to find the gauge transformation between $\mathrm{L}_{1} \mathrm{~S}(\partial-r)$ and $\mathrm{L}_{3}$. This reduces to the gauge equivalence between $\mathrm{L}_{1} \mathrm{~S}(\partial-r)$ and $\mathrm{L}_{3}$, and that can be found using the last paragraph on how to find the gauge equivalence.

\section{Conclusion}

Let us consider two irreducible third-order linear differential operators $\mathrm{L}, \mathrm{M} \in k(x)[\partial]$ such that $\mathrm{M} \longrightarrow_{E G} \mathrm{~L}$. To find the exp-product and gauge transformation parameters we proceed as follow:

1. We compute the set $\mathbb{S}$ of non-apparent singularities of L.

2. We compute for all $p \in \mathbb{S}, i=1,2,3$ the difference $e_{p}^{i}=e_{p}^{i}(\mathrm{~L})-e_{p}^{i}(\mathrm{M})$ where $e_{p}^{i}(\mathrm{~L})$ and $e_{p}^{i}(\mathrm{M})$ are the $i^{t h}$ generalized exponent of $\mathrm{L}$ and $\mathrm{M}$ at $p$, respectively.

3. We compute the exp-product parameter $r$ using the relation

$$
r=\sum_{p \in \mathbb{S} \backslash\{\infty\}} \frac{e_{p}^{i}}{t_{p}}-t_{\infty} \cdot \overline{e_{\infty}^{i}}+\sum_{p \in \mathbb{S} \backslash\{\infty\}} \frac{c_{p}}{n_{p}} t_{p}^{-1}
$$

where $\overline{e_{\infty}^{i}}=e_{\infty}^{i}-\operatorname{const}\left(e_{\infty}^{i}\right)$ with $\operatorname{const}\left(e_{\infty}^{i}\right)$ the constant term of $e_{\infty}^{i}, n_{p}=$ $\max \left\{n_{e_{p}^{i}(\mathrm{~L})}, i=1,2,3\right\}$ with $n_{e_{p}^{i}(\mathrm{~L})}$ the ramification index of $e_{p}^{i}(\mathrm{~L})$, and $c_{p} \in \mathbb{Z}$ such that $\left|c_{p}\right|<n_{p}$.

4. We compute the middle operator $\mathrm{L}_{1} \in k(x)[\partial]$ such that $\mathrm{M} \stackrel{r}{\longrightarrow} \mathrm{L}_{1} \longrightarrow_{G} \mathrm{~L}$ which is given by $\mathrm{L}_{1}=\mathrm{M}(\mathrm{S})(\partial-r)$. There exists in Maple an algorithm called symmetry (see [20], [19] and [26]) to compute the symmetric product between two differential operators.

5. We compute the operator $\mathrm{G} \in k(x)[\partial]$ of order two such that $\mathrm{L}=\mathrm{GL}_{1}$. That can be done in Maple by the algorithm called Homomorphisms (see [17] and [32]). 
6. Let $\mathrm{G}=a_{2} \partial^{2}+a_{1} \partial+a_{0}$ with $a_{0}, a_{1}, a_{2} \in k(x)$. We deduce the gauge parameters $r_{0}, r_{1}, r_{2} \in k(x)$ by taking

$$
\left\{\begin{array}{l}
r_{0}=a_{0}, \\
r_{1}=a_{1}, \\
r_{2}=a_{2} .
\end{array}\right.
$$

We have succeeded by writing and implementing in Maple algorithms for projective equivalence called Equi vExpgaugeBesSq1, Equi vExpgaugeBesSqRoot1, EquivExpgauge2F2, EquivExpgauge1F2, EquivExpgaugeOF2, EquivExpgauge1F1sq when one involved operator is coming from the operator $\mathrm{L}_{\breve{B}_{\nu}^{2}}$ with $f=g^{2}$ and $g \in k(x)$, the $\mathrm{L}_{\breve{B}_{\nu}^{2}}$ with $f \neq g^{2}$ and $g \in k(x), \mathrm{L}_{22}, \mathrm{~L}_{12}, \mathrm{~L}_{02}, \mathrm{~L}_{11}^{2}$, respectively. Those algorithms take as input two thirdorder irreducible linear differential operators $\mathrm{L}_{1}$ and $\mathrm{L}_{2}$, and return as output, if they are equivalent, a list of two elements:

- the first is the exp-product parameter $r$ such that $\mathrm{L}_{1} \stackrel{r}{\longrightarrow}_{E} \mathrm{~A} \longrightarrow_{E G} \mathrm{~L}_{2}$ where $\mathrm{A}$ is an operator in $k(x)[\partial]$;

- the second is a list containing a basis where we can take an operator $\mathrm{G}$ of order two such that $\mathrm{A}=\mathrm{L}_{2} \mathrm{G}$. Let $\mathrm{G}=a_{2}(x) \partial^{2}+a_{1}(x) \partial+a_{0}(x) \partial$, we deduce the gauge parameters $r_{0}, r_{1}$ and $r_{2}$ by taking

$$
\left\{\begin{array}{l}
r_{0}=a_{0}(x), \\
r_{1}=a_{1}(x), \\
r_{2}=a_{2}(x)
\end{array}\right.
$$

If $\mathrm{L}_{1}$ and $\mathrm{L}_{2}$ are not equivalent, the algorithm returns an empty list.

\section{Examples}

(1) Let $\mathrm{L}_{1}$ be the Bessel square root operator $\mathrm{L}_{\check{B}_{\nu}^{2}}$ with parameter $\nu=3, \mathrm{~L}_{2}$ the operator coming from $\mathrm{L}_{1}$ by the exp-product transformation with parameter $r=x-1$ and $\mathrm{L}_{3}$ the operator coming from $\mathrm{L}_{2}$ by the gauge transformation with parameters $r_{0}=$ $0, r_{1}=x$ and $r_{2}=0$.

$$
\begin{aligned}
& >\mathrm{L} 1:=2 * \mathrm{x}^{\wedge} 2 * \mathrm{Dx}^{\wedge} 3+6 * \mathrm{x} * \mathrm{Dx} \wedge 2+(-2 * \mathrm{x}-16) * \mathrm{Dx}-1 \text {; } \\
& L 1:=2 x^{2} D x^{3}+6 x D x^{2}+(-2 x-16) D x-1 \\
& >\mathrm{r}:=\mathrm{x}-1 \\
& r:=x-1 \\
& >\quad \mathrm{L} 2:=\text { ExpProduct }(\mathrm{L} 1, \mathrm{r}) \text {; } \\
& L 2:=2 x^{2} D x^{3}-6\left(x^{2}-x-1\right) x D x^{2}+\left(6 x^{4}-12 x^{3}-12 x^{2}+10 x-16\right) D x \\
& -2 x^{5}+6 x^{4}+6 x^{3}-14 x^{2}+14 x-17 \\
& >\mathrm{r} 0:=0 \text {; } \\
& r 0:=0 \\
& >\mathrm{r} 1:=\mathrm{x} \\
& r 1:=x
\end{aligned}
$$


$>\mathrm{r} 2:=0$;

$$
\begin{aligned}
& r 2:=0 \\
& >\text { L3:=GaugeTransf (L2, r0, r1, r2); } \\
& L 3:=2 x^{3}\left(2 x^{5}-6 x^{4}-6 x^{3}+14 x^{2}-14 x+17\right) D x^{3}-2\left(6 x^{7}-24 x^{6}+6 x^{5}\right. \\
& \left.+48 x^{4}-90 x^{3}+93 x^{2}-37 x-34\right) x^{2} D x^{2}+2\left(-30 x^{8}+30 x^{7}+40 x^{6}\right. \\
& \left.+6 x^{9}-112 x^{5}+195 x^{4}+40 x^{3}-335 x^{2}+225 x-153\right) x D x+24 x^{10} \\
& -36 x^{9}-20 x^{8}+92 x^{7}-204 x^{6}-88 x^{5}+686 x^{4}-1624 x^{3}+1186 x^{2} \\
& -4 x^{11}-793 x+306 \\
& >\text { B2:= Singular }(\mathrm{L} 2,\{\}) \text {; } \\
& B 2:=[[x, 0],[\infty, \infty]] \\
& >\mathrm{S} 2:=\operatorname{NotAppSing}(\mathrm{L} 2, \mathrm{~B} 2,\{\}) \text {; } \\
& S 2:=[[x, 0],[\infty, \infty]] \\
& >\quad \text { C2: =IrrRegAppsingBesSqRoot (L2, t , B2, \{\}): } \\
& >\text { EquivExpgaugeBesSqRoot1 (L1, L2, C2, x, t , T },\{\}) \text {; } \\
& \begin{array}{c}
\left\{\left[\left[\frac{x^{2}-x-3}{x}\right],\left[x^{3}\right]\right],\left[\left[\frac{x^{2}-x+3}{x}\right],\left[\frac{1}{x^{3}}\right]\right],\left[\left[\frac{x^{2}-x+6}{x}\right],\left[\frac{1}{x^{6}}\right]\right],\right. \\
\left.\left[\left[\frac{(x+2)(x-3)}{x}\right],\left[x^{6}\right]\right],[[x-1],[1]]\right\}
\end{array} \\
& >\text { B3: }=\text { Singular }(\mathrm{L} 3,\{\}) \text {; } \\
& B 3:=\left[\left[x^{5}-3 x^{4}-3 x^{3}+7 x^{2}-7 x+17 / 2, \operatorname{RootOf}\left(2{ }_{-} Z^{5}-6{ }_{-} Z^{4}\right.\right.\right. \\
& \left.\left.\left.-6 Z_{-} Z^{3}+14_{-} Z^{2}-14_{-} Z+17\right)\right],[x, 0],[\infty, \infty]\right] \\
& >\text { S3: }=\operatorname{NotAppSing}(L 3, B 3,\{\}) \text {; } \\
& S 3:=[[x, 0],[\infty, \infty]] \\
& >\text { C3: }=\operatorname{IrrRegAppsingBesSqRoot~}(\mathrm{L} 3, \mathrm{t}, \mathrm{B} 3,\{\}) \text { : } \\
& >\text { EquivExpgaugeBesSqRoot1 (L2, L3, C3, x, t }, \mathrm{T},\{\} \text { ); } \\
& \left\{\left[\left[\frac{1}{x}\right],\left[D x-\frac{1}{x}\right]\right],\left[\left[-\frac{6}{x}\right],\left[x^{7} D x+6 x^{6}\right]\right],\left[\left[-\frac{3}{x}\right],\left[x^{4} D x+3 x^{3}\right]\right],\right. \\
& {\left[\left[-\frac{2}{x}\right],\left[x^{3} D x+2 x^{2}\right]\right],\left[\left[\frac{3}{x}\right],\left[\frac{D x}{x^{2}}-\frac{3}{x^{3}}\right]\right],\left[\left[\frac{4}{x}\right],\left[\frac{D x}{x^{3}}-\frac{4}{x^{4}}\right]\right],} \\
& \left.\left[\left[\frac{6}{x}\right],\left[\frac{D x}{x^{5}}-\frac{6}{x^{6}}\right]\right]\right\} \\
& \left\{\left[\left[\frac{x^{2}-x+1}{x}\right],\left[D x-\frac{1}{x}\right]\right],\left[\left[\frac{x^{2}-x-3}{x}\right],\left[x^{4} D x+3 x^{3}\right]\right],\left[\left[\frac{x^{2}-x+3}{x}\right]\right.\right. \\
& \left.\left[\frac{D x}{x^{2}}-\frac{3}{x^{3}}\right]\right],\left[\left[\frac{x^{2}-x+4}{x}\right],\left[\frac{D x}{x^{3}}-\frac{4}{x^{4}}\right]\right],\left[\left[\frac{x^{2}-x+6}{x}\right],\left[\frac{D x}{x^{5}}-\frac{6}{x^{6}}\right]\right], \\
& {\left[\left[\frac{(x+1)(x-2)}{x}\right],\left[x^{3} D x+2 x^{2}\right]\right],\left[\left[\frac{(x+2)(x-3)}{x}\right],\left[x^{7} D x+6 x^{6}\right]\right] \text {, }} \\
& [[x-1],[x D x]]\}
\end{aligned}
$$


(2) Let $\mathrm{L}_{1}$ be the same operator as in (1), $\mathrm{L}_{2}$ the operator coming from $\mathrm{L}_{1}$ by the exp-product transformation with parameter $r=(x-1)(x-7)$ and $\mathrm{L}_{3}$ the operator coming from $\mathrm{L}_{2}$ by the gauge transformation with parameters $r_{0}=x-9, r_{1}=0$ and $r_{2}=0$.

$>\mathrm{r}:=(\mathrm{x}-1) *(\mathrm{x}-7)$;

$$
r:=(x-1)(x-7)
$$

$>\quad \mathrm{L} 2:=$ ExpProduct $(\mathrm{L} 1, \mathrm{r})$;

$L 2:=2 x^{2} D x^{3}-6\left(x^{3}-8 x^{2}+7 x-1\right) x D x^{2}+\left(6 x^{6}-96 x^{5}+468 x^{4}-696 x^{3}\right.$

$\left.+438 x^{2}-86 x-16\right) D x+48 x^{7}-426 x^{6}+1714 x^{5}-3222 x^{4}+3290 x^{3}$

$-2 x^{8}-1710 x^{2}+228 x+111$

$>\mathrm{r} 0:=\mathrm{x}-9$;

$$
r 0:=x-9
$$

$>\mathrm{r} 1:=0$

$$
r 1:=0
$$

$>\mathrm{r} 2:=0$

$$
r 2:=0
$$

> L3: =GaugeTransf (L2,r0,r1, r2);

$$
\begin{aligned}
L 3:= & 2 x^{2}\left(2 x^{9}-48 x^{8}-1864 x^{6}+4560 x^{5}-8354 x^{4}+9752 x^{3}-8528 x^{2}\right. \\
& \left.+432 x^{7}+4211 x-1296\right) D x^{3}-2\left(-192 x^{11}+2490 x^{10}-16956 x^{9}\right. \\
& +6 x^{12}+67248 x^{8}-171918 x^{7}+319920 x^{6}-425946 x^{5}+413743 x^{4} \\
& \left.-284040 x^{3}+128063 x^{2}-35638 x+3888\right) x D x^{2}+\left(12 x^{15}-480 x^{14}\right. \\
& -76440 x^{12}-1657216 x^{10}+4333048 x^{9}-8391000 x^{8}+12203078 x^{7} \\
& +8136 x^{13}+440652 x^{11}-13401932 x^{6}+11005272 x^{5}-6540700 x^{4} \\
& \left.+2623026 x^{3}-593486 x^{2}+22068 x+20736\right) D x-4 x^{17}-4020 x^{15} \\
& +192 x^{16}-369996 x^{13}-6928124 x^{11}+18406312 x^{10}-8411426 x^{3} \\
& +48304 x^{14}+1910192 x^{12}-37207068 x^{9}+58247804 x^{8}+242081 x \\
& -71057310 x^{7}+67146884 x^{6}-48140538 x^{5}+1220934 x^{2}-125568 \\
& +24996944 x^{4}
\end{aligned}
$$

$>$ B2: $=$ Singular $(\mathrm{L} 2,\{\})$;

$$
B 2:=[[x, 0],[\infty, \infty]]
$$

$>$ S2: $=\operatorname{NotAppSing}(\mathrm{L} 2, \mathrm{~B} 2,\{\})$;

$$
S 2:=[[x, 0],[\infty, \infty]]
$$

$>\mathrm{C} 2:=\operatorname{IrrRegAppsingBesSqRoot}(\mathrm{L} 2, \mathrm{t}, \mathrm{B} 2,\{\}):$

$>$ EquivExpgaugeBesSqRoot1 ( $\mathrm{L} 1, \mathrm{~L} 2, \mathrm{C} 2, \mathrm{x}, \mathrm{t}, \mathrm{T},\{\})$;

$$
\begin{gathered}
\{[(x-1)(x-7)],[1]],\left[\left[\frac{x^{3}-8 x^{2}+7 x-6}{x}\right],\left[x^{6}\right]\right],\left[\left[\frac{x^{3}-8 x^{2}+7 x-3}{x}\right],\right. \\
\left.\left.\left[x^{3}\right]\right],\left[\left[\frac{x^{3}-8 x^{2}+7 x+3}{x}\right],\left[\frac{1}{x^{3}}\right]\right],\left[\left[\frac{x^{3}-8 x^{2}+7 x+6}{x}\right],\left[\frac{1}{x^{6}}\right]\right]\right\}
\end{gathered}
$$

$>$ B3:= Singular $(\mathrm{L} 3,\{\})$; 


$$
\begin{aligned}
B 3:=[ & {[x, 0],\left[x^{9}-24 x^{8}+216 x^{7}-932 x^{6}+2280 x^{5}-4177 x^{4}+4876 x^{3}\right.} \\
& -4264 x^{2}+\frac{4211 x}{2}-648, \operatorname{RootOf}\left(2{ }_{-} Z^{9}-48_{-} Z^{8}+432 \_Z^{7}\right. \\
& -1864 \_Z^{6}+4560{ }_{-} Z^{5}-8354_{-} Z^{4}+9752_{-} Z^{3}-8528_{-} Z^{2} \\
& \left.\left.\left.+4211_{-} Z-1296\right)\right],[\infty, \infty]\right]
\end{aligned}
$$

$>$ S3: $=\operatorname{NotAppSing}(\mathrm{L} 3, \mathrm{~B} 3,\{\})$;

$$
S 3:=[[x, 0],[\infty, \infty]]
$$

$>\mathrm{C} 3:=\operatorname{IrrRegAppsingBesSqRoot}(\mathrm{L} 3, \mathrm{t}, \mathrm{B} 3,\{\}):$

$>$ EquivExpgaugeBesSqRoot1 (L2, L3, C3, x, t , T, \{\});

$$
\begin{gathered}
\left\{\left[\left[-\frac{6}{x}\right],\left[x^{7} D x+x^{7}-3 x^{6}\right]\right],\left[\left[-\frac{3}{x}\right],\left[x^{4} D x+x^{4}-6 x^{3}\right]\right],\right. \\
\left.\left[\left[\frac{3}{x}\right],\left[\frac{D x}{x^{2}}+\frac{x-12}{x^{3}}\right]\right],\left[\left[\frac{6}{x}\right],\left[\frac{D x}{x^{5}}+\frac{x-15}{x^{6}}\right]\right]\right\}
\end{gathered}
$$

$>$ EquivExpgaugeBesSqRoot1(L1, L3, C3, x, t , T, \{\}) ;

$$
\begin{gathered}
\left\{[[(x-1)(x-7)],[x D x+x-9]],\left[\left[\frac{x^{3}-8 x^{2}+7 x-6}{x}\right],\right.\right. \\
\left.\left[x^{7} D x+x^{7}-3 x^{6}\right]\right],\left[\left[\frac{x^{3}-8 x^{2}+7 x-3}{x}\right],\left[x^{4} D x\right.\right. \\
\left.\left.\quad+x^{4}-6 x^{3}\right]\right],\left[\left[\frac{x^{3}-8 x^{2}+7 x+3}{x}\right],\left[\frac{D x}{x^{2}}+\frac{x-12}{x^{3}}\right]\right], \\
\left.\left[\left[\frac{x^{3}-8 x^{2}+7 x+6}{x}\right],\left[\frac{D x}{x^{5}}+\frac{x-15}{x^{6}}\right]\right]\right\}
\end{gathered}
$$




\section{Chapter 4}

\section{Bessel Square Root Type Solutions}

Let $\mathrm{L}$ be an irreducible third-order linear differential operator that we want to solve in terms of Bessel square root functions. In this chapter, we apply the theories developed in the last chapter to discuss the details of the algorithm to find Bessel square root type solutions of $\mathrm{L}$ if they exist. Using what we have done before, with $\mathrm{L}_{0}=\mathrm{L}_{\breve{B}_{\nu}^{2}}$ (as in the last chapter) we just have to consider

$$
\mathrm{L}_{\check{B}_{\nu}^{2}} \stackrel{f}{\longrightarrow} C \mathrm{M} \longrightarrow_{E G} \mathrm{~L} .
$$

The operator $\mathrm{L}$ is the only input to the algorithm. We define $k$ to be an extension field of $\mathbb{Q}$ which is algebraically closed and has characteristic zero such that $\mathrm{L} \in \mathrm{K}[\partial]$ with $\mathrm{K}=k(x)$.

As we have said in the last chapter, we will just take a closer look at the part $\mathrm{L}_{\check{B}_{\nu}^{2}} \stackrel{f}{\longrightarrow} C$ M. Once we found the Bessel parameter $\nu$ and the parameter $f$ we can obtain M from $\mathrm{L}_{\check{B}_{\nu}^{2}}$. For fixed $\mathrm{M} \in \mathrm{K}[\partial]$ we know how to solve, by the last chapter, the question of equivalence between $\mathrm{M}$ and L. We can then finally solve (4.1).

Note that $f$ and $\nu$ are related. If we can fix $f$ then we can get a finite list of candidates for $\nu$ by using the exponents differences at the zeroes of $f$. On the other hand, if we know $\nu$ we can identify disappearing singularities and it will give us the information about the multiplicity orders of the factors of $f$.

In order to find Bessel square root type solutions (solutions in terms of $\check{\mathrm{B}}_{\nu}^{2}(x)=\mathrm{B}_{\nu}^{2}(\sqrt{x})$ functions), we will deal with $\sqrt{f}$ where $f \in k(x)$ is the change of variable parameter. Therefore, we will consider two cases:

1. when $f=g^{2}$ with $g \in k(x)$,

2. when $f \neq g^{2}$ with $g \in k(x)$.

In this chapter, for $n \in \mathbb{N} \backslash\{0\}$ and $a$ an element of $\mathrm{K}=k(x)$ or not, when we will talk about $a$ modulo $\frac{1}{n} \mathbb{Z}$, that will mean $a$ modulo an additive element of $\frac{1}{n} \mathbb{Z}$.

\subsection{Change of variable parameters are squares of ratio- nal functions in $k(x)$}

Let us denote by $\mathrm{L}_{B_{\nu}^{2}}$ the operator associated to the Bessel square function $\mathrm{B}_{\nu}^{2}$ :

$$
\mathrm{L}_{B_{\nu}^{2}}=x^{2} \partial^{3}+3 x \partial^{2}+\left(1-4 x^{2}-4 \nu^{2}\right) \partial-4 x .
$$


The operator $\mathrm{L}_{\breve{B}_{\nu}^{2}}$ can be derived from $\mathrm{L}_{B_{\nu}^{2}}$ by the transformation $\sqrt{x}$ :

$$
\mathrm{L}_{B_{\nu}^{2}} \stackrel{\sqrt{x}}{\longrightarrow} C \mathrm{~L}_{\check{B}_{\nu}^{2}}
$$

Since $f=g^{2}$ with $g \in k(x)$, then the transformation

$$
\mathrm{L}_{\check{B}_{\nu}^{2}} \stackrel{f}{\longrightarrow} C \mathrm{M} \longrightarrow_{E G} \mathrm{~L}
$$

is equivalent to the transformation

$$
\mathrm{L}_{B_{\nu}^{2}} \stackrel{g}{\longrightarrow} C \mathrm{M} \longrightarrow{ }_{E G} \mathrm{~L} .
$$

Therefore, in the whole section we will consider the situation

$$
\mathrm{L}_{B_{\nu}^{2}} \stackrel{f}{\longrightarrow} C \mathrm{M} \longrightarrow_{E G} \mathrm{~L}
$$

instead of (4.1), and solve now in terms of solutions of $\mathrm{L}_{B_{\nu}^{2}}$ that means find Bessel square type solutions. To get Bessel square root solutions (solutions in terms of $\check{\mathrm{B}}_{\nu}^{2}(x)=\mathrm{B}_{\nu}^{2}(\sqrt{x})$ ), we will just replace the change of variable parameter $f$, in the solutions that we have found in terms of $\mathrm{B}_{\nu}^{2}$, by its square.

Remarks 4.1. 1. The operator $\mathrm{L}_{B_{\nu}^{2}}$ is irreducible when $\nu \notin \frac{1}{2}+\mathbb{Z}$, and has two singularities: one regular at $x=0$ and another irregular at $x=\infty$.

2. $\nu \in \mathbb{Z}$ is the necessary and sufficient condition for $\mathrm{L}_{B_{\nu}^{2}}$ to have logarithmic solutions at $x=0$.

3. All the theorems, lemmas, corollaries and remarks that we have seen in chapter two and chapter three related to the operator $\mathrm{L}_{\check{B}_{\nu}^{2}}$ are also valid for the operator $\mathrm{L}_{B_{\nu}^{2}}$.

4. The parts, in chapter three, called "Observations" and "Assumptions" hold also for $\mathrm{L}_{0}=\mathrm{L}_{B_{\nu}^{2}}$.

\subsubsection{Exponent differences}

The generalized exponents of $\mathrm{L}_{B_{\nu}^{2}}$ at 0 and $\infty$ are

$$
\begin{aligned}
& >\quad \operatorname{gen} \_\exp (\mathrm{LBB}, \mathrm{t}, \mathrm{x}=0) ; \\
& {[[0, t=x],[-2 \nu, t=x],[2 \nu, t=x]]} \\
& >\quad \text { gen_exp }(\mathrm{LBB}, \mathrm{t}, \mathrm{x}=\inf \text { inity }) ; \\
& {\left[\left[1, t=x^{-1}\right],\left[-2 t^{-1}+1, t=x^{-1}\right],\left[2 t^{-1}+1, t=x^{-1}\right]\right]}
\end{aligned}
$$

Hence the ramification index of $\mathrm{L}_{B_{\nu}^{2}}$ at all the points is 1 .

Using the assumptions of the last chapter, the generalized exponents of $\mathrm{M}$ at

- a zero $p$ of $f$ are

$$
\left[0, \quad-2 m_{p} \nu, \quad 2 m_{p} \nu\right]
$$


- a pole $p$ of $f$ are

$$
\left[m_{p}, \quad m_{p}+2 \sum_{j=-m_{p}}^{-1} j \bar{f}_{1, j+m_{p}} t_{p}^{j}, \quad m_{p}-2 \sum_{j=-m_{p}}^{-1} j \bar{f}_{1, j+m_{p}} t_{p}^{j}\right]
$$

where $\left\{\begin{aligned} f & =t_{p}^{-m_{p}} \sum_{j=0}^{+\infty} f_{j-m_{p}} t_{p}^{j}, \quad \text { with } f_{j-m_{p}} \in k \text { and } f_{-m_{p}} \neq 0, \\ \sum_{j=0}^{+\infty} f_{j-m_{p}} t_{p}^{j} & =\sum_{j=0}^{+\infty} \bar{f}_{1, j} t_{p}^{j} \quad \text { with } \bar{f}_{1, j} \in k .\end{aligned}\right.$

$\sum_{j=0}^{+\infty} f_{j-m_{p}} t_{p}^{j}=\sum_{j=0}^{+\infty} \bar{f}_{1, j} t_{p}^{j} \Longrightarrow \bar{f}_{1, j+m_{p}}=f_{j} \quad \forall j \in \mathbb{N}$. Therefore, the generalized exponents of $\mathrm{M}$ at a pole $p$ of $f$ are

$$
\left[m_{p}, \quad m_{p}+2 \sum_{j=-m_{p}}^{-1} j f_{j} t_{p}^{j}, \quad m_{p}-2 \sum_{j=-m_{p}}^{-1} j f_{j} t_{p}^{j}\right] .
$$

So the ramification index of $\mathrm{M}$ at all the points is 1 . Since the exp-product and gauge transformations don't change the ramification index, the ramification index of $\mathrm{L}$ at all the points has also to be 1 , otherwise L can't have Bessel square type solutions. $\mathrm{M}$ at

Using (4.5) and (4.6) we have that the exponents differences, modulo a factor -1 , of

- a zero $p$ of $f$ are

$$
\left[-2 m_{p} \nu, \quad 2 m_{p} \nu, \quad 4 m_{p} \nu\right]
$$

- a pole $p$ of $f$ are

$$
\left[2 \sum_{j=-m_{p}}^{-1} j f_{j} t_{p}^{j}, \quad-2 \sum_{j=-m_{p}}^{-1} j f_{j} t_{p}^{j}, \quad-4 \sum_{j=-m_{p}}^{-1} j f_{j} t_{p}^{j}\right]
$$

By using now the fact that the exponent difference is invariant modulo $\frac{1}{n} \mathbb{Z}$ (here $n=1$ ) under the exp-product and gauge transformations, the exponent differences of $\mathrm{L}$ at

- a zero $p$ of $f$ are

$$
\left[-2 m_{p} \nu+\alpha_{1}, \quad 2 m_{p} \nu+\alpha_{2}, \quad 4 m_{p} \nu+\alpha_{3}\right]
$$

with $\alpha_{1}, \alpha_{2}, \alpha_{3} \in \mathbb{Z}$

- a pole $p$ of $f$ are

$$
\left[\beta_{1}+2 \sum_{j=-m_{p}}^{-1} j f_{j} t_{p}^{j}, \quad \beta_{2}-2 \sum_{j=-m_{p}}^{-1} j f_{j} t_{p}^{j}, \quad \beta_{3}-4 \sum_{j=-m_{p}}^{-1} j f_{j} t_{p}^{j}\right]
$$

with $\beta_{1}, \beta_{2}, \beta_{3} \in \mathbb{Z}$. 
Since one of the main tool of our work are exponent differences and for the reason of generalization, we will consider the first two exponent differences. The third one will be used as a necessary condition to test if we are solving in terms of Bessel square functions.

Definition 4.2. Let us consider the situation in (4.4). We define the exponent differences of $\mathrm{L}$ at

- a zero $p$ of $f$ by

$$
\Delta(\mathrm{L}, p)= \pm 2 m_{p} \nu \bmod \mathbb{Z}
$$

- a pole $p$ of $f$ by

$$
\Delta(\mathrm{L}, p)= \pm 2 \sum_{j=-m_{p}}^{-1} j f_{j} t_{p}^{j} \bmod \mathbb{Z} .
$$

Since we know how to find all the poles of $f$ (using Corollary 3.13) which are elements of the set $\mathrm{S}_{\text {irr }}(\mathrm{L})$, and also their multiplicity orders (using their exponent differences), we can start in order to find $f$, to first find its polar part at all its poles. By summing them, we will have $f$ up to a constant (see Definition 3.26).

\subsubsection{Parameter $f$ up to a constant}

Since, by Corollary 3.13, $\mathrm{S}_{\mathrm{irr}}(\mathrm{L})$ contains all the poles of $f$, we can always find candidates for the parameter $f$ up to a constant using the set $\mathrm{S}_{\text {irr }}(\mathrm{L})$ and the exponent differences at its elements: that is the polar part of $f$. The polar part of $f$ at a pole $p$ is given by $\sum_{j=-m_{p}}^{-1} f_{j} t_{p}^{j}$ where $m_{p}$ is the multiplicity order of $p$.

Let us have a look on the exponent differences of $\mathrm{L}$ at a pole of $f$ in (4.12). It can be brought in the form

$$
\beta+\sum_{j=-m_{p}}^{-1} a_{j} t_{p}^{j}, \text { with } \beta \in \mathbb{Z}
$$

where $a_{j}= \pm 2 j f_{j}$. So the polar part of $f$, modulo a factor -1 , at a pole $p$ is

$$
\sum_{j=-m_{p}}^{-1} \frac{a_{j}}{2 j} t_{p}^{j}
$$

Maple's output for the generalized exponents is not ordered. Hence it will be difficult to order the exponent differences. They will be defined modulo a factor -1 . So the polar part of $f$ at a pole $p$ given by (4.13) will appear modulo a factor -1 using Maple. Therefore, we will have two candidates for the polar part of $f$ at any of its poles. By choosing at each pole one candidate and doing the summation of all of them, we obtain a candidate for $f$ up to a constant (candidate of the polar part of $f$ ).

We have implemented in Maple an algorithm called BesSquarSubst for finding the candidates for $f$ up to a constant.

Let us denote by $\mathcal{F}$ the set of candidates for $f$ up to a constant. We know how to find the parameter $f$ up to a constant. The problem is how to find this constant. Knowing a zero of $f$ (element of $\mathrm{S}_{\mathrm{reg}}(\mathrm{L})$ ) can be helpful. Hence we will distinguish two cases: when $\mathrm{S}_{\mathrm{reg}}(\mathrm{L}) \neq \emptyset$ and when $\mathrm{S}_{\mathrm{reg}}(\mathrm{L})=\emptyset$. 


\subsubsection{No zero of $f$ is known $\left(\mathrm{S}_{\mathrm{reg}}(\mathrm{L})=\emptyset\right)$}

Here we have absolutely no information about the zeroes of $f$ and $\Delta(\mathrm{L}, s) \in \mathbb{Z}$ for all $s \notin \mathrm{S}_{\text {irr }}(\mathrm{L})$. What we know is just the candidates for the polar part of $f$. Those candidates are the candidates for $f$ modulo a constant $c \in k$.

Definition 4.3. For $m \in \mathbb{N}$, we can define

$$
\mathrm{N}(m)=\left\{\frac{j}{2 m} \mid 0 \leq j \leq 2 m-1\right\} .
$$

For $a=\frac{a_{1}}{a_{2}} \in \mathbb{Q}$ with $\operatorname{gcd}\left(a_{1}, a_{2}\right)=1$, let us denote numer $(a)=a_{1}$ and $\operatorname{denom}(a)=a_{2}$. We will see by the proof of the following lemma that, for $p=\operatorname{denom}(2 \nu), \mathrm{N}(p)$ gives us some information about the Bessel square parameter $\nu$ : the Bessel square parameter appears modulo an additive integer in $\mathrm{N}(p)$.

Lemma 4.4. Consider the situation in (4.4) with $\mathrm{S}_{\text {reg }}(\mathrm{L})=\emptyset$. Let $n$ be the degree of the numerator of $f$ and $p=\operatorname{denom}(2 \nu)$. Then $\nu \in \mathrm{N}(p)$ modulo $\mathbb{Z}$ and $p$ divides $n$.

Proof. For $s \notin \mathrm{S}_{\text {irr }}(\mathrm{L})$, we have $\Delta(\mathrm{L}, s) \in \mathbb{Z}$ since $\mathrm{S}_{\text {reg }}(\mathrm{L})=\emptyset$. Therefore, by Definition 4.2, $\nu \in \mathbb{Q}$ and we can find $a, p \in \mathbb{N}$ and $z \in \mathbb{Z}$ such that

$$
\nu=z+\frac{a}{2 p}, \quad 0<a \leq 2 p-1, \operatorname{gcd}(a, p)=1
$$

Therefore, $\nu \in \mathrm{N}(p)$ modulo $\mathbb{Z}$.

Let $s$ be a zero of $f$ with multiplicity order $m_{s}$. Since taking $\nu$ or $-\nu$ doesn't change our operator $\mathrm{L}_{B_{\nu}^{2}}$, we can assume by (4.11) that $\Delta(\mathrm{L}, s)=2 m_{s} \nu \bmod \mathbb{Z}$. Hence we have

$$
\begin{aligned}
& \Delta(\mathrm{L}, s)=2 m_{s} \nu+b \in \mathbb{Z} \text { with } b \in \mathbb{Z} \\
\Longrightarrow & 2 m_{s}\left(z+\frac{a}{2 p}\right)+b \in \mathbb{Z} \Longrightarrow \frac{m_{s} a}{p} \in \mathbb{Z} \\
\Longrightarrow & p \text { divides } m_{s} \text { since } \operatorname{gcd}(a, p)=1 .
\end{aligned}
$$

Let $n$ be the degree of the numerator of $f$. Since $p$ divides all the multiplicity orders of the zeroes of $f$ then $p=\operatorname{denom}(2 \nu)$ divides $n$. Hence the numerator of $f$ has to be a $p$-th power.

Let $g$ be a candidates for $f$. Then $g=g_{1}+c$ where $g_{1}$ is a candidate for the polar part of $f$. We know how to find $g_{1}$ by our algorithm BesSquarSubst but we don't know the value of $c$. That means we don't know the degree $n$ of the numerator of $g$. In order to find $\nu$, the problem now remains how to find this degree $n$ without knowing the constant part $c$ of $g$.

Lemma 4.5. Consider the situation in (4.4) with $\mathrm{S}_{\text {reg }}(\mathrm{L})=\emptyset$ and $\nu=z+\frac{a}{2 p}$ for some $a, p \in \mathbb{Z}$ and $\operatorname{gcd}(a, p)=1$.

(a)- If $\infty \in \mathrm{S}_{\text {irr }}(\mathrm{L})$, then $\operatorname{deg}($ numer $(f))=\operatorname{deg}($ numer $(f+c)) \quad \forall c \in \mathbb{C}$.

(b)- If $\infty \notin \mathrm{S}_{\text {irr }}(\mathrm{L})$, then $p \mid \operatorname{deg}($ numer $(f)) \Leftrightarrow p \mid \operatorname{deg}(\operatorname{denom}(f))$.

Proof. Similar as the proof of [11, Lemma 3.16]. 
Corollary 4.6. Consider the situation in (4.4) with $\mathrm{S}_{\text {reg }}(\mathrm{L})=\emptyset$. Let us define

$$
n= \begin{cases}\operatorname{deg}(\text { numer }(g+c)) & \text { if } \infty \in \mathrm{S}_{\text {irr }}(\mathrm{L}) \\ \operatorname{deg}(\operatorname{denom}(g+c)) & \text { otherwise }\end{cases}
$$

where $g$ is the polar part of $f$. Then the set of divisors of $\operatorname{deg}($ numer $(f))$ is the set of divisors of $n$.

Proof. Just use Lemma 4.5 above.

Corollary 4.7. Consider the situation in (4.4) with $\mathrm{S}_{\text {reg }}(\mathrm{L})=\emptyset$ and $n$ as in Corollary 4.6 above. Then $n \geq 2$.

Proof. Let $p=\operatorname{denom}(2 \nu)$. By Lemma 4.4, $p \mid \operatorname{deg}(\operatorname{numer}(f))$ and then, using Corollary 4.6, we will also have $p \mid n . g$ polar part of $f$ implies $f=g+c$ and $g=f_{1} / f_{2}+f_{3}$ with $c \in k$, $f_{1}, f_{2}, f_{3} \in k[x]$ and $\operatorname{deg}\left(f_{1}\right)<\operatorname{deg}\left(f_{2}\right)$ or $f_{1}=0$.

1. Let us show that $n \geq 1$.

(a) Let us assume $\infty \in \mathrm{S}_{\text {irr }}(\mathrm{L})$ and $m_{\infty}$ its multiplicity order as a pole of $f$. Then $f_{3} \in k[x] \backslash k\left(f_{3}\right.$ is the polar part of $f$ at $\left.\infty\right)$ and $m_{\infty} \geq 1$.

$$
\begin{array}{rlr}
\operatorname{deg}(\operatorname{numer}(f)) & =\operatorname{deg}(\operatorname{numer}(g+c))=\operatorname{deg}\left(\text { numer }\left(f_{1} / f_{2}+f_{3}+c\right)\right) \\
& =\operatorname{deg}\left(\operatorname{numer}\left(f_{1} / f_{2}+f_{3}\right)\right) & \text { since } f_{3} \in k[x] \backslash k \text { and } \\
& & \operatorname{deg}\left(f_{1}\right)<\operatorname{deg}\left(f_{2}\right) \text { or } f_{1}=0 \\
& =\operatorname{deg}(\operatorname{numer}(g))=n .
\end{array}
$$

$\infty$ pole of $f$ implies $\operatorname{deg}(\operatorname{numer}(f))=\operatorname{deg}(\operatorname{denom}(f))+m_{\infty}$ and hence we have $\operatorname{deg}($ numer $(f)) \geq 1$ since $m_{\infty} \geq 1$. Therefore, by using (4.15), we have $n \geq 1$.

(b) Let us assume $\infty \notin \mathrm{S}_{\text {irr }}(\mathrm{L})$. Then $f_{3}=0$ i.e $g=f_{1} / f_{2}+c$

$$
\begin{aligned}
\mathrm{S}_{\text {irr }}(\mathrm{L}) \neq \emptyset \text { and } \infty \notin \mathrm{S}_{\text {irr }}(\mathrm{L}) & \Rightarrow f_{1} \neq 0 \text { i.e. } \operatorname{deg}\left(f_{2}\right) \geq 1 \text { since } \operatorname{deg}\left(f_{1}\right)<\operatorname{deg}\left(f_{2}\right) \\
& \Rightarrow n=\operatorname{deg}(\operatorname{denom}(g))=\operatorname{deg}\left(f_{2}\right) \geq 1
\end{aligned}
$$

2. Let us show that $n \neq 1$.

Let us assume $n=1 . p \mid n$ implies $p \mid 1$. That means $2 \nu \in \mathbb{Z}$ i.e $\nu \in \mathbb{Z}$ or $\nu \in 1 / 2+\mathbb{Z}$. Therefore we are in the logarithmic case or $\mathrm{L}_{B_{\nu}^{2}}$ is reducible, that means $\mathrm{S}_{\mathrm{reg}}(\mathrm{L}) \neq \emptyset$ or $\mathrm{L}_{B_{\nu}^{2}}$ is reducible. All of that is not true. Hence $n \neq 1$.

We know now how to find the degree $n$ of the numerator of a candidate $g=g_{1}+c$ of $f$ with $c \in k g_{1}$ a candidate for the polar part of $f$. So we can find its associated candidates for the Bessel square parameter $\nu$.

Conclusion:

To find candidates for $(f, \nu)$, we proceed as follow:

1. We compute the polar part of $f$ : that can be done by our algorithm BesSquarSubst.

Hence we get candidates for $f$ modulo a an additive constant. 
2. Let $g$ be a candidate for the polar part of $f$ ( $g$ is an element in the list giving by the algorithm BesSquarSubst). Then we have to find, if it exists, $c \in k$ such that $f=g+c$.

(a) We take $n$ as in Corollary 4.6:

$$
n= \begin{cases}\operatorname{deg}(\operatorname{numer}(g+c)) & \text { if } \infty \in \mathrm{S}_{\mathrm{irr}}(\mathrm{L}) \\ \operatorname{deg}(\operatorname{denom}(g+c)) & \text { otherwise }\end{cases}
$$

(b) $\operatorname{denom}(2 \nu)$ must divide $n$ (by the proof of Lemma 4.4): if $n=1$ then $\operatorname{denom}(2 \nu)$ will also be 1 and that means $\nu=\frac{z}{2}$ with $z \in \mathbb{Z}$. That is not good since we will be in the logarithmic case $(\nu \in \mathbb{Z})$ or $\mathrm{L}_{B_{\nu}^{2}}$ will be a reducible operator $\left(\nu=z_{1}+\frac{1}{2}\right.$ with $\left.z_{1} \in \mathbb{Z}\right)$. Hence $n>1$. For $n \leq 1$, we will say that $g+c$, $\forall c \in k$, is not a good candidate for $f$ and then we will return to step 2., and take another candidate $g$ for the polar part of $f$.

(c) We are not in the logarithmic case so $\nu \notin \mathbb{Z}$. Hence denom $(2 \nu) \neq 1$. If not that will means $\nu=z+\frac{1}{2}$ with $z \in \mathbb{Z}$ and therefore $\mathrm{L}_{B_{\nu}^{2}}$ is a reducible operator. Thus $\operatorname{denom}(2 \nu) \geq 2$.

(d) Since $\operatorname{denom}(2 \nu) \geq 2$, we take all the divisors $p$ of $n$ greater or equal to 2. Let $\mathcal{C}$ be the set of those divisors.

(e) Let $p \in \mathcal{C}$ (a divisor of $n$ ). We check whether for certain constants $c \in k$ the monic part of the numerator of $g+c$ becomes a $p$-th power. This can simply be done with linear algebra and leads to a non-linear system of equations for the constant $c$. Solving these equations gives us a set $\mathrm{C}_{p}$ of possible values for $c$ related to $p$. If $\mathrm{C}_{p}=\emptyset, p$ is not a good candidate for $\operatorname{denom}(2 \nu)$ and then we will return and take another element $p$ in $\mathcal{C}$.

i. $\forall c \in \mathrm{C}_{p}$, compute $g+c$. They will give us the list of candidates $\mathrm{F}_{p}$ for $f$ related to $p$.

ii. Compute $\mathrm{N}(p)$.

iii. Remove in $\mathrm{N}(p)$

A. all the modulo integers which appear for an element.

B. all the integers and the elements in the form $z+\frac{1}{2}$ with $z \in \mathbb{Z}$

Let us call again $\mathrm{N}(p)$ the final set of $\mathrm{N}(p)$ after all the modifications. $\left[\mathrm{F}_{p}, \mathrm{~N}(p)\right]$ will be a list related to $p$ such that the first element is the list of candidates for $f$ and the second their associated candidates for $\nu$. All those elements $\left[\mathrm{F}_{p}, \mathrm{~N}(p)\right]$ generate a set $\mathcal{W}$.

$\mathcal{W}$ will represent all the candidates for $(f, \nu)$.

We have implemented in Maple an algorithm called findBesselvfInt to get those candidates for $(f, \nu)$, if they exist. An example for this case can be found in our Maple worksheet associated to this thesis. 


\subsubsection{Some zeroes of $f$ are known $\left(\mathrm{S}_{\mathrm{reg}}(\mathrm{L}) \neq \emptyset\right)$}

For a fixed candidate $g$ of the polar part of $f(g \in \mathcal{F})$, we can find the constant $c$ such that $f=g+c$ using an element of $\mathrm{S}_{\text {reg }}(\mathrm{L})$. This can be done by our implemented algorithm in Maple called changeconstantBesSq. But the other elements of $\mathrm{S}_{\mathrm{reg}}(\mathrm{L})$ must also be the zeroes of $g+c$, otherwise we will exclude $g$ from the list of candidates for $f$ up to a constant $(\mathcal{F})$. This test can also be done by one of our implemented algorithms in Maple called CandichangvarBesSq. So we have a list of possible candidates for $f$.

Since $f$ and $\nu$ are related, let us now see how to get candidates for $\nu$. This will help us also to reduce the number of candidates for $f$.

Lemma 4.8. Consider (4.4). Then the following statements are equivalent:

(a)- The Bessel square parameter is an integer, i.e. $\nu \in \mathbb{Z}$.

(b)- There is $s \in \mathrm{S}_{\text {reg }}(\mathrm{L})$ such that $\mathrm{L}$ is logarithmic at $s$.

Proof. Using Lemma 3.7 in [11], our lemma will be true if we can replace the Bessel operator $\mathrm{L}_{B_{\nu}}$ by our operator $\mathrm{L}_{B_{\nu}^{2}}$. The key is the fact that $\mathrm{L}_{B_{\nu}}$ has a logarithmic solution at $x=0$ if and only if $\nu \in \mathbb{Z}$. Since $B_{\nu}^{2}$ (solutions of $\mathrm{L}_{B_{\nu}^{2}}$ ) are the squares of $B_{\nu}$ (solutions of $\mathrm{L}_{B_{\nu}}$ ), we can apply Lemma 3.7 (in [11]) to $\mathrm{L}_{B_{\nu}^{2}}$.

Remark 4.9. If there exists $s \in \mathrm{S}_{\text {reg }}(\mathrm{L})$ at which $\mathrm{L}$ is logarithmic then $\mathrm{L}$ is logarithmic at every element of $\mathrm{S}_{\text {reg }}(\mathrm{L})$. Moreover, if there exists one zero of the parameter $f$ at which $\mathrm{L}$ is logarithmic then $\mathrm{L}$ will be logarithmic at all the zeroes of $f$. That means all the zeroes of $f$ are exactly the singularities $\mathrm{S}_{\text {reg }}(\mathrm{L})$.

We will distinguish here two cases for finding $\nu$ :

1. the logarithmic case: when $\nu \in \mathbb{Z}$,

2. the non-logarithmic case: when $\nu \notin \mathbb{Z}$.

\section{Logarithmic case}

By Remark 4.9, $\mathrm{S}_{\text {reg }}(\mathrm{L})$ represents all the zeroes of $f$. Since in this case $\nu \in \mathbb{Z}$ and using the invariance of $\mathbb{C}(x) B_{\nu}^{2^{\prime \prime}}+\mathbb{C}(x) B_{\nu}^{2^{\prime}}+\mathbb{C}(x) B_{\nu}^{2}$ under $\nu \mapsto-\nu$ and $\nu \mapsto \nu+1$, we can take zero as the Bessel parameter $(\nu=0)$. But that can generate gauge transformations which may not be needed. Therefore, to simplify the output, we will proceed as follows to find the Bessel parameter:

1. For every $P_{s} \in \overline{\mathrm{S}_{\mathrm{reg}}(\mathrm{L})}$, let $m_{s}$ be the multiplicity order of its associated $s \in \mathrm{S}_{\mathrm{reg}}(\mathrm{L})$ as zero of $f$. Since taking $\nu$ or $-\nu$ doesn't change the Bessel square operator $\mathrm{L}_{B_{\nu}^{2}}$ then

$$
\Delta(\mathrm{L}, s)=2 m_{s} \nu+\beta_{s} \text { with } \beta_{s} \in \mathbb{Z}
$$

and we have as set of candidates for $\nu$ related to $s$ :

$$
\begin{aligned}
\mathrm{N}_{s} & =\left\{\frac{\Delta(\mathrm{L}, s)+j_{s}}{2 m_{s}} \mid j_{s} \in \mathbb{N} \text { and } 0 \leq j_{s}<2 m_{s}\right\} \\
& =\left\{\frac{\left.\left(\Delta(\mathrm{L}, s)+j_{s}\right) \cdot \operatorname{deg}\left(P_{s}\right)\right)}{2 m_{s} \cdot \operatorname{deg}\left(P_{s}\right)} \mid j_{s} \in \mathbb{N} \text { and } 0 \leq j_{s}<2 m_{s}\right\} .
\end{aligned}
$$


Since taking again $\nu$ or $-\nu$ doesn't change the Bessel square operator $\mathrm{L}_{B_{\nu}^{2}}$ then we can take $\mathrm{N}_{s}$ as

$$
\begin{aligned}
\mathrm{N}_{s} & =\left\{\left|\frac{\left.\left(\Delta(\mathrm{L}, s)+j_{s}\right) \cdot \operatorname{deg}\left(P_{s}\right)\right)}{2 m_{s} \cdot \operatorname{deg}\left(P_{s}\right)}\right| \mid j_{s} \in \mathbb{N} \text { and } 0 \leq j_{s}<2 m_{s}\right\} \\
& =\left\{\frac{\left|\Delta(\mathrm{L}, s)+j_{s}\right| \cdot \operatorname{deg}\left(P_{s}\right)}{2 m_{s} \cdot \operatorname{deg}\left(P_{s}\right)} \mid j_{s} \in \mathbb{N} \text { and } 0 \leq j_{s}<2 m_{s}\right\} .
\end{aligned}
$$

2. We take $n=\sum_{P_{s} \in \overline{\mathrm{S}_{\text {reg }}(\mathrm{L})}} 2 m_{s} \cdot \operatorname{deg}\left(P_{s}\right)$. Hence

$$
\begin{aligned}
& n=\left\{\begin{array}{lc}
\operatorname{deg}\left(\operatorname{numer}\left(f^{2}\right)\right)+2 m_{\infty} & \text { if } \infty \in \mathrm{S}_{\text {reg }}(\mathrm{L}), \\
\operatorname{deg}\left(\operatorname{numer}\left(f^{2}\right)\right) & \text { otherwise }
\end{array}\right. \\
& =\left\{\begin{array}{l}
\operatorname{deg}\left(\operatorname{denom}\left(f^{2}\right)\right) \text { if } \infty \in \mathrm{S}_{\mathrm{reg}}(\mathrm{L}), \\
\operatorname{deg}\left(\operatorname{numer}\left(f^{2}\right)\right) \text { otherwise }
\end{array}\right. \\
& =\max \left(\operatorname{deg}\left(\operatorname{numer}\left(f^{2}\right)\right), \operatorname{deg}\left(\operatorname{denom}\left(f^{2}\right)\right)\right) \text {. }
\end{aligned}
$$

3. We choose for $P_{s} \in \overline{\mathrm{S}_{\mathrm{reg}}(\mathrm{L})}$ one element $\mathrm{N}_{s}$. With those chosen elements we can compute

$$
a=\left(\sum_{P_{s} \in \overline{\mathrm{S}_{\mathrm{reg}}(\mathrm{L})}}\left|\Delta(\mathrm{L}, s)+j_{s}\right| \cdot \operatorname{deg}\left(P_{s}\right)\right) \cdot \frac{1}{\sum_{P_{s} \in \overline{\mathrm{S}_{\mathrm{reg}}(\mathrm{L})}} 2 m_{s} \cdot \operatorname{deg}\left(P_{s}\right)}
$$

where $j_{s} \in \mathbb{N}$ with $0 \leq j_{s}<2 m_{s} \forall P_{s} \in \overline{\mathrm{S}_{\text {reg }}(\mathrm{L})}$. Since all the $j_{s}$ are positive, we have

$$
a \leq\left(\sum_{P_{s} \in \overline{\mathrm{S}_{\text {reg }}(\mathrm{L})}}\left(|\Delta(\mathrm{L}, s)|+j_{s}\right) \cdot \operatorname{deg}\left(P_{s}\right)\right) \cdot \frac{1}{\sum_{P_{s} \in \overline{\mathrm{S}_{\text {reg }}(\mathrm{L})}} 2 m_{s} \cdot \operatorname{deg}\left(P_{s}\right)} .
$$

Using equation (4.16) we get

$$
a \leq\left(\sum_{P_{s} \in \overline{\mathrm{Sreg}}(\mathrm{L})}|\Delta(\mathrm{L}, s)| \cdot \operatorname{deg}\left(P_{s}\right)\right) \cdot \frac{1}{n}+\left[\sum_{P_{s} \in \overline{\mathrm{Sreg}}(\mathrm{L})} j_{s} \cdot \operatorname{deg}\left(P_{s}\right)\right] \cdot \frac{1}{n} .
$$

Since $\forall P_{s} \in \overline{\mathrm{S}_{\text {reg }}(\mathrm{L})}$ we have $0 \leq j_{s}<2 m_{s}$,

$$
\begin{aligned}
& \sum_{P_{s} \in \overline{\mathrm{S}_{\mathrm{reg}}(\mathrm{L})}} j_{s} \cdot \operatorname{deg}\left(P_{s}\right)<\sum_{P_{s} \in \overline{\mathrm{S}_{\mathrm{reg}}(\mathrm{L})}} 2 m_{s} \cdot \operatorname{deg}\left(P_{s}\right) \\
\Longrightarrow & {\left[\sum_{P_{s} \in \overline{\mathrm{S}_{\mathrm{reg}}(\mathrm{L})}} j_{s} \cdot \operatorname{deg}\left(P_{s}\right)\right] \cdot \frac{1}{n}<1 . }
\end{aligned}
$$

Using (4.17),(4.18) we get

$$
a \leq\left(\sum_{P_{s} \in \overline{\mathrm{S}_{\mathrm{reg}}(\mathrm{L})}} \Delta(\mathrm{L}, s) \cdot \operatorname{deg}\left(P_{s}\right)\right) \cdot \frac{1}{n} .
$$


4. For $e \in \mathbb{Q}$ let us denote by "floor $(e)$ " the integer least or equal to $e$. By the fact that $\nu \in \mathbb{Z}$ and in order to simplify the output, it is better to take as candidate for $\nu$ the floor of $a$ :

$$
\nu=\text { floor }\left(\left[\sum_{P_{s} \in \overline{\mathrm{S}_{\mathrm{reg}}(\mathrm{L})}} \Delta(\mathrm{L}, s) \cdot \operatorname{deg}\left(P_{s}\right)\right] \cdot \frac{1}{n}\right) \text {. }
$$

We have implemented in Maple an algorithm called $f$ indBesselvfln to get both $(\nu, f)$ candidates, if they exist. An example for this case can be found in our Maple worksheet associated to this thesis.

\section{Non-logarithmic case}

When we have candidates for $f$, we have also all the multiplicity orders of the elements of $\mathrm{S}_{\mathrm{reg}}(\mathrm{L})$ as zeroes of $f$. Using now the exponent differences of $\mathrm{L}$ at the elements of $\mathrm{S}_{\mathrm{reg}}(\mathrm{L})$ given by (4.11), we can get $\nu$ modulo an integer.

Definition 4.10. Consider (4.4) and $s \in \mathrm{S}_{\text {reg }}(\mathrm{L})$. Let $m_{s}$ be the multiplicity order of $s$ as a zero of $f$. We define

$$
\begin{aligned}
\mathrm{N}_{s} & =\left\{\frac{\Delta(\mathrm{L}, s)+j}{2 m_{s}} \mid 0 \leq j \leq 2 m_{s}-1\right\} \\
\text { and } \mathrm{N} & =\left\{\nu \in k \backslash \mathbb{Z} \mid \forall s \in \mathrm{S}_{\text {reg }}(\mathrm{L}), \exists \alpha_{s} \in \mathbb{Z}: \quad \nu+\alpha_{s} \in \mathrm{N}_{s}\right\} .
\end{aligned}
$$

For every singularity $s \in \mathrm{S}_{\text {reg }}(\mathrm{L})$ the Bessel square parameter $\nu$ appears in $\mathrm{N}_{s}$ modulo some integer.

Lemma 4.11. Consider (4.4) and assume $\mathrm{S}_{\text {reg }}(\mathrm{L}) \neq \emptyset$. Then there exists some integer $z \in \mathbb{Z}$ such that $\nu+z \in \mathrm{N}$. That means $\mathrm{N}$ is the set of candidates for $\nu$ modulo $\mathbb{Z}$.

Proof. Let $s \in \mathrm{S}_{\text {reg }}(\mathrm{L})$. Since taking $\nu$ or $-\nu$ doesn't change the Bessel square operator $\mathrm{L}_{B_{\nu}^{2}}$, we get $\Delta(\mathrm{L}, s)=2 m_{s} \nu+l_{s}$ with $l_{s} \in \mathbb{Z}$. Hence $\nu=\frac{\Delta(\mathrm{L}, s)-l_{s}}{2 m_{s}}$. We can always rewrite $l_{s}=\bar{l}_{s}+2 m_{s} \times \alpha_{s}$ with $\bar{l}_{s}, \alpha_{s} \in \mathbb{Z}$ and $0 \leq \bar{l}_{s} \leq 2 m_{s}-1$. Therefore,

$$
\nu=\frac{\Delta(\mathrm{L}, s)+\bar{l}_{s}}{2 m_{s}}+\alpha_{s} \text { with } \bar{l}_{s}, \alpha_{s} \in \mathbb{Z} \text { and } 0 \leq \bar{l}_{s} \leq 2 m_{s}-1
$$

and we have

$$
\nu-\alpha_{s} \in \mathrm{N}_{s} .
$$

Hence $\exists z_{s} \in \mathbb{Z}$ such that $\nu+z_{s} \in \mathrm{N}_{s}$. In this way, we find such an integer $z_{s}$ for every $s \in \mathrm{S}_{\text {reg }}(\mathrm{L})$ such that $\nu+z_{s} \in \mathrm{N}_{s}$. From the definition of $\mathrm{N}$ it follows that $\nu+z \in \mathrm{N}$ for some $z \in \mathbb{Z}$.

Since $\mathbb{C}(x) B_{\nu}^{2^{\prime \prime}}+\mathbb{C}(x) B_{\nu}^{2^{\prime}}+\mathbb{C}(x) B_{\nu}^{2}$ is invariant under $\nu \mapsto \nu+1$ and $\nu \mapsto-\nu$ we only need to find $\nu$ modulo an integer and a factor -1 . So we can regard $\mathrm{N}$ as a set of candidates for $\nu$. 


\section{How to improve the set $\mathrm{N}$ of candidates for $\nu$}

By the definition of $\mathrm{N}$ we have

$$
\mathrm{N}=\bigcap_{s \in \mathrm{S}_{\mathrm{reg}}(\mathrm{L})}\left[\mathrm{N}_{s} \text { modulo } \mathbb{Z} \text { or a factor }-1\right]
$$

1. Because of the invariance of $\mathbb{C}(x) B_{\nu}^{2^{\prime \prime}}+\mathbb{C}(x) B_{\nu}^{2^{\prime}}+\mathbb{C}(x) B_{\nu}^{2}$ under $\nu \mapsto-\nu$, we remove in $\mathrm{N}$ all the negative elements.

2. Because of the invariance of $\mathbb{C}(x) B_{\nu}^{2^{\prime \prime}}+\mathbb{C}(x) B_{\nu}^{2^{\prime}}+\mathbb{C}(x) B_{\nu}^{2}$ under $\nu \mapsto \nu+1$, we remove in $\mathrm{N}$ all the modulo an integer equivalents of its elements.

3. Because we are not in the logarithmic case, we remove all the integers in $\mathrm{N}$.

4. Because $\mathrm{L}_{B_{\nu}^{2}}$ has to be an irreducible operator, we also remove in $\mathrm{N}$ all the elements of the form $\frac{1}{2}+z$ with $z \in \mathbb{Z}$.

So the new set $\mathrm{N}$ that we will get at the end will be the improved set of candidates for $\nu$.

To reduce the set of candidates for $(\nu, f)$, we have to distinguish between three cases:

1. rational case: when $\Delta(\mathrm{L}, s) \in \mathbb{Q}$ for all $s \in \mathrm{S}_{\text {reg }}(\mathrm{L})$. That means $\nu \in \mathbb{Q}$.

2. basefield case: when there exists $s \in \mathrm{S}_{\text {reg }}(\mathrm{L})$ with $\Delta(\mathrm{L}, s) \in k \backslash \mathbb{Q}$. That means $\nu \in k \backslash \mathbb{Q}$.

3. irrational case: when there exists $s \in \mathrm{S}_{\text {reg }}(\mathrm{L})$ with $\Delta(\mathrm{L}, s) \notin k$. That means $\nu \notin k$.

\section{Rational case}

Let $g$ be a candidate for $f$ and $\mathrm{N}$ its associated candidates for $\nu$ given by our algorithm findcand3. Let $h=\frac{\operatorname{numer}(\mathrm{g})}{\prod_{s \in \mathrm{S}_{\mathrm{reg}}(\mathrm{L})}(x-s)^{m_{s}}}$ where $m_{s}$ is the multiplicity order of the zero $s \in \mathrm{S}_{\text {reg }}(\mathrm{L})$ of $f . h$ doesn't have zeroes in $\mathrm{S}_{\text {reg }}(\mathrm{L})$, so we are in the integer case with $f=h$. Therefore, by taking $n$ as the degree of $h$ we will have:

- if $n \leq 1$ then we cannot reduce our set $\mathrm{N}$ of candidates for $\nu$;

- if $n \geq 2$ then a similar argument as in the case $\mathrm{S}_{\text {reg }}(\mathrm{L})=\emptyset$ (integer case) is used since each zero $s$ of $h$ satisfies $\Delta_{i}(\mathrm{~L}, s) \in \mathbb{Z}(i=1,2,3)$ and L is not logarithmic at $s$. Hence, for $v \in \mathrm{N}$, the denominator $p$ of $2 v$ must divide $n$. If not, our candidate $v$ for $\nu$ is not good and we have to remove it from N. Hence, the monic part of the polynomial $h$ must be a $p$-th power.

Conclusion:

To reduce the set of candidates for $(f, \nu)$ we proceed as follow:

1. We take an element $(g, N)$ where $g$ is a candidate for $f$ and $\mathrm{N}$ the set of its associated candidates for $\nu$. These conditions are given by our algorithm findcand3. 
2. We compute $h=\frac{\text { numer }(\mathrm{g})}{\prod_{s \in \mathrm{S}_{\mathrm{reg}}(\mathrm{L})}(x-s)^{m_{s}}}$ where $m_{s}$ is the multiplicity order of the zero $s \in \mathrm{S}_{\mathrm{reg}}(\mathrm{L})$ of $f$.

3. We compute the monic part of $h$.

4. For each element $v \in \mathrm{N}$, we check if the monic part of $h$ is a $p$-th power with $p=\operatorname{denom}(2 v)$. If the result is true then $v$ is good. If not, we take another $v$ in $\mathrm{N}$ and repeat the same procedure. All those elements $v$ for which this is true generate a set $\mathcal{W}$.

5. $(g, \mathcal{W})$ will be the reduced set of candidates of $(f, \nu)$.

We have implemented in Maple an algorithm for this reduction called findBesselvfRat. An example for this case can be found in our Maple worksheet associated to this thesis.

\section{Basefield case}

That is the case where $\nu \in k \backslash \mathbb{Q}$. Then every zero $s$ of $f$ must, by (4.11), satisfy $\Delta(\mathrm{L}, s) \in k \backslash \mathbb{Q}$. That means $s \in \mathrm{S}_{\text {reg }}(\mathrm{L})$ (for $s$ to be a regular point or an apparent singularity we need $\left.\Delta_{i}(\mathrm{~L}, s) \in \mathbb{Q}, i=1,2,3\right)$. So $\mathrm{S}_{\text {reg }}(\mathrm{L})$ represents all the zeros of $f$.

To reduce the set of candidates for $(f, \nu)$, we use the following statement about the multiplicity orders of the zeroes of $f$.

Lemma 4.12. Consider (4.4). Let $\nu \in k \backslash \mathbb{Q}, \mathrm{S}_{\text {reg }}(\mathrm{L})=\left\{s_{1}, \ldots, s_{n}\right\}$ and $d_{i}=\Delta\left(\mathrm{L}, s_{i}\right), i=$ $1, \ldots, n$. Then we can do the following steps:

1- Compute $r_{i}, t_{i} \in \mathbb{Q}$ such that $d_{i}=r_{i} d_{1}+t_{i}$.

2- Let $a_{i}, b_{i} \in \mathbb{Z}$ be such that $r_{i}=\frac{a_{i}}{b_{i}}$ and $\operatorname{gcd}\left(a_{i}, b_{i}\right)=1$.

3- Let $l=\operatorname{lcm}\left(b_{i}, 1 \leq i \leq n\right)$.

Then the monic part of the numerator of $f$ is a power of $h \in k[x]$ where

$$
h=\prod_{i=1}^{n}\left(x-s_{i}\right)^{l r_{i}} .
$$

Proof. The proof is similar to the proof of Lemma 3.19 in [11].

Conclusion:

To reduce the set of candidates for $(f, \nu)$, we proceed as follow:

1. Let $\mathrm{S}_{\mathrm{reg}}(\mathrm{L})=\left\{s_{1}, \ldots, s_{n}\right\}$, with $s_{i} \in k \quad \forall i \in\{1, \ldots, n\}$.

2. Take one element in $\mathrm{S}_{\text {reg }}(\mathrm{L})$. We have taken for example $s_{1}$.

3. Let $d_{i}=\Delta\left(\mathrm{L}, s_{i}\right), i=1, \ldots, n$.

(a) Compute $r_{i} \in \mathbb{Q}, i=1, \ldots, n$, such that $d_{i}=r_{i} d_{1}+t_{i}$ where $t_{i} \in \mathbb{Q}$. This can be done in Maple with the algorithm called "compare" (see [11] and [42]).

(b) Compute $l=\operatorname{lcm}\left(\operatorname{denom}\left(r_{i}\right), i=1, \ldots, n\right)$. 
(c) If $\infty \in \mathrm{S}_{\mathrm{reg}}(\mathrm{L})$ then take 1 as its minimal polynomial: $1 \equiv x-\infty$ or $1 \equiv \frac{1}{x}$.

(d) Compute $h=\prod_{i=1}^{n}\left(x-s_{i}\right)^{l r_{i}}$.

4. Take an element $\left(g, \mathrm{~N}_{g}\right)$ where $g$ is a candidate for $f$ and $\mathrm{N}_{g}$ the set of its associated candidates for $\nu$..

5. Define

$$
a=\left\{\begin{array}{lr}
1 & \text { if } \operatorname{deg}(h)=0, \\
\frac{\operatorname{deg}(\operatorname{numer}(g))}{\operatorname{deg}(h)} & \text { otherwise. }
\end{array}\right.
$$

If $a \notin \mathbb{N}_{g}$ then $\left(g, \mathrm{~N}_{g}\right)$ is not a good element, we have to go in 4., and take another $\left(g, \mathrm{~N}_{g}\right)$.

6. Define: $g_{1}$ to be the monic part of $g$ and $h_{1}$ the monic part of $h$.

7. If $g_{1}-h_{1}^{a} \neq 0$ then $\left(g, \mathrm{~N}_{g}\right)$ is not a good element and we have to go to step 4 . and take another $\left(g, \mathrm{~N}_{g}\right)$. All the pairs $\left(g, \mathrm{~N}_{g}\right)$ for which $g_{1}-h_{1}^{a}=0$ generate a set called E.

E will be the reduced set for the candidates for $(f, \nu)$.

We have implemented in Maple an algorithm for this reduction called findBesSqveBasfield. An example for this case can be found in our Maple worksheet associated to this thesis.findBesSqviBasfield

\section{Irrational case}

Lemma 4.13. In the irrational case, we know all the zeroes of $f$.

Proof. A change of variables can transfer a regular singularity to a removable singularity only if $\nu \in \mathbb{Q} \backslash \mathbb{Z}(\nu \notin \mathbb{Z}$ because we are not in the logarithmic case $)$. Since $\longrightarrow_{E G}$ only changes the exponent differences by an element of $\frac{1}{n} \mathbb{Z}$ (invariance modulo $\frac{1}{n} \mathbb{Z}$, here $n=1)$ then $\longrightarrow_{E G}$ can also transfer a regular singularity to a removable singularity only if $\nu \in \mathbb{Q} \backslash \mathbb{Z}$. So in the irrational case $(\nu \notin k), \mathrm{S}_{\text {reg }}(\mathrm{L})$ contains all the zeroes of $f$.

To reduce the set of candidates for $(\nu, f)$ we proceed as follows:

1. For all $s \in \mathrm{S}_{\text {reg }}(\mathrm{L})$, we find the extension $\operatorname{ext}(s)$ of $k$ using the expression of the exponent differences of $\mathrm{L}$ at $s$. Then we define

$$
\operatorname{ext} 1=\left(\bigcap_{s \in \mathrm{S}_{\mathrm{reg}}(\mathrm{L})} \operatorname{ext}(s)\right) \backslash k .
$$

If ext $1=\emptyset$ then we are in the basefield case and we can use our algorithm $\mathrm{f}$ indBesSqvf vfBasfield to find, if they exist, candidates for $(\nu, f)$. Since we have $\nu \notin k$ and $\nu$ appears in the expression of the exponent differences of $L$ at any $s \in \mathrm{S}_{\text {reg }}(\mathrm{L})$, then $\operatorname{ext} 1 \neq \emptyset$. 
2. We choose one element $\sigma$ of ext1. For all $s \in \mathrm{S}_{\mathrm{reg}}(\mathrm{L})$, let $\sigma_{s}$ be the leading coefficient of the exponent differences of $\mathrm{L}$ at $s$ with respect to the variable $\sigma$. Let $m_{s}$ be the multiplicity order of $s$ as zero of $f$. Since we work with $\Delta(\mathrm{L}, s)= \pm 2 m_{s} \nu+z_{s}$ with $z_{s} \in \mathbb{Z}$ then

$$
\sigma_{s}=2 m_{s} \beta \quad \forall s \in \mathrm{S}_{\mathrm{reg}}(\mathrm{L})
$$

where $\beta \in \operatorname{ext} 1 \cup k$ is independent of $s$.

3. For all $s \in \mathrm{S}_{\text {reg }}(\mathrm{L})$ let $P_{s}$ be the minimal polynomial associated to $s$. Hence the set $\left\{P_{s_{1}}, \ldots, P_{s_{m}}\right\}$ of all those minimal polynomials has $m$ elements with $m \leq n$ where $n$ is the number of elements in $\mathrm{S}_{\mathrm{reg}}(\mathrm{L})$. Let

$$
b_{P_{s_{i}}}=\sigma_{s_{i}} \operatorname{deg}\left(P_{s_{i}}\right) \quad \forall i \in\{1, \ldots, m\} .
$$

4. For all $i \in\{1, \ldots, m\}$, we can define the following rational numbers $r_{i}$

$$
r_{i}=\left\{\begin{array}{cc}
1 & \text { if } i=1, \\
\left|\frac{b_{P_{s_{i}}}}{b_{P_{s_{1}}}}\right| & \text { otherwise }
\end{array} \quad \Rightarrow r_{i}=\left\{\begin{array}{cc}
1 & \text { if } i=1 \\
\left|\frac{m_{s_{i}} \cdot \operatorname{deg}\left(P_{s_{i}}\right)}{m_{s_{1}} \cdot \operatorname{deg}\left(P_{s_{1}}\right)}\right| & \text { otherwise, }
\end{array}\right.\right.
$$

and denote by $\widetilde{r}_{i}$ their denominator: $\widetilde{r}_{i}=\operatorname{denom}\left(r_{i}\right)$.

5. Let us define $l=\operatorname{lcm}\left(\widetilde{r}_{i}, 1 \leq i \leq m\right)$. Then $r_{i} \cdot l \in \mathbb{N}$ and $l \mid m_{s_{1}} \cdot \operatorname{deg}\left(P_{s_{1}}\right)$ since $\widetilde{r}_{i} \mid m_{s_{1}} \cdot \operatorname{deg}\left(P_{s_{1}}\right)$ for all $1 \leq i \leq m$. Therefore, $r_{i} \cdot l \mid m_{s_{i}} \cdot \operatorname{deg}\left(P_{s_{i}}\right)$ for all $i \in\{1, \ldots, m\}$ since by $(4.23)$ we have $m_{s_{i}} \cdot \operatorname{deg}\left(P_{s_{i}}\right)=r_{i} \cdot m_{s_{1}} \cdot \operatorname{deg}\left(P_{s_{1}}\right)$.

6. For all $i \in\{1, \ldots, m\}$, let $q_{i}=\frac{m_{s_{i}} \cdot \operatorname{deg}\left(P_{s_{i}}\right)}{r_{i} \cdot l} \in \mathbb{N}$. Hence, by replacing $r_{i}$ by its value in (4.23), we have $q_{i}=\frac{m_{s_{1}} \cdot \operatorname{deg}\left(P_{s_{1}}\right)}{l}=q$ which is independent of $i \in\{1, \ldots, m\}$. So

$$
m_{s_{i}} \cdot \operatorname{deg}\left(P_{s_{i}}\right)=\left(r_{i} \cdot l\right) q \quad i \in\{1, \ldots, m\}
$$

7. Let $h=\prod_{i \in\{1, \cdots, m\}} P_{s_{i}}^{\left(r_{i} \cdot l\right) / \operatorname{deg}\left(P_{s_{i}}\right)}$. Then

$$
h^{q}=\prod_{i \in\{1, \cdots, m\}} P_{s_{i}}^{\left(r_{i} \cdot l \cdot q\right) / \operatorname{deg}\left(P_{s_{i}}\right)}=\prod_{i \in\{1, \cdots, m\}} P_{s_{i}}^{\left(m_{s_{i}} \cdot \operatorname{deg}\left(P_{s_{i}}\right)\right) / \operatorname{deg}\left(P_{s_{i}}\right)}=\prod_{i \in\{1, \cdots, m\}} P_{s_{i}}^{m_{s_{i}}}
$$

Hence $h^{q}$ is the monic part of the numerator of $f$. That means the numerator of $f$ must be a scalar multiple of a $q^{\text {th }}$ power of $h$.

8. For every candidate of $(\nu, f)$, we check if the numerator of $f$ is a scalar multiple of a $q^{\text {th }}$ power of $h$. If it is the case, we keep $(\nu, f)$ in our list of candidates, if it is not, we just remove it from our list of candidates.

We have implemented in Maple an algorithm for this reduction called findBesSqIrr. An example for this case can be found in our Maple worksheet associated to this thesis. 


\subsubsection{Example}

We have written some algorithms (depending on many cases) on how to find Bessel square type solutions, if they exist, with change of variable parameter $f \in \mathrm{K} \backslash k$. We have also summarize them by writing another algorithm called BesSqSolutions which takes as input an third-order linear differential operator L and returns, if they exist, all the parameters of transformations $\left(r, r_{2}, r_{1}, r_{0}, f \in k(x)\right)$ and also the Bessel square parameter $\nu \in k$ such that we are in situation (4.4)

$$
\mathrm{L}_{B_{\nu}^{2}} \stackrel{f}{\longrightarrow} C \mathrm{M} \longrightarrow \longrightarrow_{E G} \mathrm{~L} .
$$

If not it will return "No Bessel square type solutions". This algorithm deals with all possible cases.

We will take here just one example and show explicitly how some of our algorithms work.

Let us consider the Bessel square operator $\mathrm{L}_{B B}$ with parameter $\nu=a \cdot \operatorname{RootOf}\left(x^{2}+\right.$ 2) $+1 / 2$

$$
>\text { LBB: }=\operatorname{subs}\left(n u=a * \operatorname{Root} 0 f\left(x^{\wedge} 2+2\right)+1 / 2, L B B\right) \text {; }
$$

$L B B:=x^{2} D x^{3}+3 x D x^{2}+\left(1-4 x^{2}-4\left(a \operatorname{RootOf}\left(Z^{2}+2\right)+1 / 2\right)^{2}\right) D x-4 x$ by

Let us apply to $\mathrm{L}_{B B}$ the change of variables transformation with parameter $f$ given

$>\mathrm{f}:=(\mathrm{x}-2)^{\wedge} 2 *(\mathrm{x}-7) /(\mathrm{x}-1)^{\wedge} 5$

$$
f:=\frac{(x-2)^{2}(x-7)}{(x-1)^{5}}
$$

That gives us the following operator called $\mathrm{L}$

$$
\begin{aligned}
& >\text { L: }=\text { ChangeOfVariables }(\text { LBB }, f) \text {; } \\
& L:=(x-2)^{2}(x-7)^{2}(x-1)^{13}\left(x^{2}-13 x+27\right)^{2} D x^{3}+3\left(x^{4}-26 x^{3}+188 x^{2}-512 x\right. \\
& +439)(x-2)(x-7)(x-1)^{12}\left(x^{2}-13 x+27\right) D x^{2}-\left(-17006112 a^{2}+5061 x^{16}\right. \\
& +8503056 \text { a RootOf }\left(Z^{2}+2\right)-28099018824 x+3 x^{18}-186 x^{17}-80970 x^{15} \\
& -6178910 x^{13}+32446722 x^{12}-126753214 x^{11}+380186954 x^{10}-923670030 x^{9} \\
& +849004 x^{14}+2035186004 x^{8}-4736476918 x^{7}+11776763148 x^{6}-26665094450 x^{5} \\
& +47608789582 x^{4}+53675365323 x^{2}-61591453506 x^{3}-32 a^{2} x^{18}+1984 a^{2} x^{17} \\
& -322654944 a^{2} x^{12}+1233427200 a^{2} x^{11}-3572058432 a^{2} x^{10}+7942409984 a^{2} x^{9} \\
& -13663419712 a^{2} x^{8}+18229147136 a^{2} x^{7}-18799770848 a^{2} x^{6}+14838366784 a^{2} x^{5} \\
& -8794541792 a^{2} x^{4}+3787568640 a^{2} x^{3}-1118974176 a^{2} x^{2}+202813632 a^{2} x \\
& +853760 a^{2} x^{15}-8788192 a^{2} x^{14}+62679104 a^{2} x^{13}+16 a \text { RootOf }\left(Z^{2}+2\right) x^{18} \\
& \text { +26992a RootOf }\left({ }_{-} Z^{2}+2\right) x^{16}-992 a \text { RootOf }\left({ }_{-} Z^{2}+2\right) x^{17}-53984 a^{2} x^{16} \\
& -7419183392 a \text { RootOf }\left(\_Z^{2}+2\right) x^{5}-101406816 a \text { RootOf }\left(Z^{2}+2\right) x \\
& -9114573568 a \text { RootOf }\left({ }_{-} Z^{2}+2\right) x^{7}+1786029216 a \text { RootOf }\left({ }_{-} Z^{2}+2\right) x^{10} \\
& +6831709856 a \text { RootOf }\left({ }_{-} Z^{2}+2\right) x^{8}+4397270896 a \text { RootOf }\left({ }_{-} Z^{2}+2\right) x^{4} \\
& -1893784320 a \text { RootOf }\left({ }_{-} Z^{2}+2\right) x^{3}-3971204992 a \operatorname{RootOf}\left({ }_{-} Z^{2}+2\right) x^{9}
\end{aligned}
$$




$$
\begin{aligned}
& -616713600 a \text { RootOf }\left({ }_{-} Z^{2}+2\right) x^{11}+559487088 a \text { RootOf }\left({ }_{-} Z^{2}+2\right) x^{2} \\
& +4394096 a \text { RootOf }\left({ }_{-} Z^{2}+2\right) x^{14}+161327472 a \text { RootOf }\left({ }_{-} Z^{2}+2\right) x^{12} \\
& +9399885424 a \text { RootOf }\left({ }_{-} Z^{2}+2\right) x^{6}-426880 a \text { RootOf }\left(Z^{2}+2\right) x^{15} \\
& \left.-31339552 a \text { RootOf }\left({ }_{-} Z^{2}+2\right) x^{13}+6668295207\right)(x-1) D x+32(x-2)^{3} \\
& \times\left(x^{2}-13 x+27\right)^{5}(x-7)
\end{aligned}
$$

Let us assume that we have as input this operator $\mathrm{L}$ and then see how with our codes we can find its Bessel square type solutions if they exist. That means if we can find the Bessel square parameter $\nu$ and the transformation parameters such that

$$
\mathrm{L}_{B B} \stackrel{f}{\longrightarrow} C \mathrm{M} \longrightarrow \longrightarrow_{E G} \mathrm{~L} .
$$

Let ext be the set of algebraic numbers, parameters and names (except the variable $x$ ) which appear in the expression of the coefficients of $\mathrm{L}$ :

$$
\begin{array}{r}
>\operatorname{ext}:=\operatorname{indets}(\mathrm{L},\{\operatorname{RootOf}, \text { name }\}) \operatorname{minus}\{\mathrm{x}, \mathrm{Dx}\} ; \\
\text { ext }:=\left\{a, \operatorname{RootOf}\left({ }_{-} Z^{2}+2\right)\right\}
\end{array}
$$

Let extp be the set of all algebraic numbers given by the RootOf expressions in ext such that all its elements are algebraic over the minimal field containing $\mathbb{Q} \cup$ (ext $\backslash$ extp). Since sometimes we can use in our computations the RootOf expression associated with its index, we will take extp $=\{\}$.

Let us denote by $\mathrm{E}$ the set of all the singularities of $\mathrm{L}$ (we can find it using our implemented code called Singular).

$>\quad \mathrm{E}:=$ Singular $(\mathrm{L}, \mathrm{ext})$ :

Then the set of non-apparent singularities of L is given by

$>\mathrm{F}:=\operatorname{NotAppSing}(\mathrm{L}, \mathrm{E}$, ext $)$;

$$
F:=[[\infty, \infty],[x-1,1],[x-2,2],[x-7,7]]
$$

Let $\mathrm{S}_{\mathrm{irr}}$ be a set of irregular singularities of $\mathrm{L}$ which are the set of poles of $f$.

$$
\begin{aligned}
>\operatorname{Sirr}:= & \operatorname{irrsingBesSq}(\mathrm{L}, \mathrm{t}, \mathrm{F}, \mathrm{ext}) ; \\
\operatorname{Sirr}:= & {\left[[[x-1,1]],\left[\left[5,-60 t^{-5}+5+104 t^{-4}-48 t^{-3}+4 t^{-2}, 60 t^{-5}+5\right.\right.\right.} \\
& \left.\left.\left.\quad-104 t^{-4}+48 t^{-3}-4 t^{-2}\right]\right],\left[\left[-60 t^{-5}+104 t^{-4}-48 t^{-3}+4 t^{-2}\right]\right],[5]\right]
\end{aligned}
$$

Let us take $\mathrm{G}$ as the set of elements in $\mathrm{F}$ at which $\mathrm{L}$ has all its exponent differences in $k$. Let $\mathrm{S}_{\text {reg }}$ be the set of elements in $\mathrm{G}$ at which $\mathrm{L}$ has logarithmic solutions or all its exponent differences are not, at same time, in $\mathbb{N}$. All the elements of $S_{\text {reg }}$ belong to the set of the zeroes of $f$ and they are given by

$>$ Sreg: =regsingtrueBesSq (L, t, $\operatorname{Sirr}[-1]$, ext $)$;

$$
\begin{aligned}
& \text { Sreg }:=\left[[[\infty, \infty],[x-2,2],[x-7,7]],\left[\left[0,-2-4 a \operatorname{RootOf}\left({ }_{-} Z^{2}+2\right),\right.\right.\right. \\
&\left.2+4 a \operatorname{RootOf}\left({ }_{-} Z^{2}+2\right)\right],\left[0,-2-4 a \operatorname{RootOf}\left({ }_{-} Z^{2}+2\right),\right. \\
&\left.2+4 a \operatorname{RootOf}\left({ }_{-} Z^{2}+2\right)\right],\left[0,-1-2 a \operatorname{RootOf}\left({ }_{-} Z^{2}+2\right),\right. \\
&\left.\left.1+2 a \operatorname{RootOf}\left({ }_{-} Z^{2}+2\right)\right]\right],\left[\left[2+4 a \operatorname{RootOf}\left({ }_{-} Z^{2}+2\right)\right],\right. \\
& {\left.\left.\left[2+4 a \operatorname{RootOf}\left({ }_{-} Z^{2}+2\right)\right],\left[1+2 a \operatorname{RootOf}\left({ }_{-} Z^{2}+2\right)\right]\right]\right] }
\end{aligned}
$$

$S_{\text {reg }}$ can be divided into two subsets: the set of non-removable singularities of $L$ (at least one exponent difference is not an integer or L has logarithmic solution(s)) and 
a set of removable singularities of $\mathrm{L}$ (all the exponent differences are integers and $\mathrm{L}$ doesn't have logarithmic solution(s)). The set of non-removable singularities of L can also be divided into two sets: the singularities at which $L$ has logarithmic solutions and the singularities at which L doesn't have logarithmic solutions. Hence, let us denote by $\mathrm{RS}_{\mathrm{reg}}$ a set of three sets: the first is the set of non-removable singularities of $\mathrm{L}$ with non-logarithmic solutions, the second is the set of removable singularities of $\mathrm{L}$ in $\mathrm{S}_{\text {reg }}$, and the third is the set of singularities of $\mathrm{L}$ with logarithmic solutions. With our code called SregseptrueBesSq we can find this set $\mathrm{RS}_{\text {reg: }}$ :

$>$ NRemSreg:= SregseptrueBesSq (L, Sreg, ext) [1];

$$
\begin{aligned}
& \text { NRemSreg }:=\left[[[\infty, \infty],[x-2,2],[x-7,7]],\left[\left[0,-2-4 a \operatorname{RootOf}\left({ }_{-} Z^{2}+2\right),\right.\right.\right. \\
& \left.2+4 a \operatorname{RootOf}\left(Z^{2}+2\right)\right],\left[0,-2-4 a \operatorname{RootOf}\left(Z^{2}+2\right)\right. \text {, } \\
& \left.2+4 a \operatorname{RootOf}\left(Z^{2}+2\right)\right],\left[0,-1-2 a \operatorname{RootOf}\left({ }_{-} Z^{2}+2\right)\right. \text {, } \\
& \left.\left.1+2 a \operatorname{RootOf}\left({ }_{-} Z^{2}+2\right)\right]\right],\left[\left[2+4 a \operatorname{RootOf}\left({ }_{-} Z^{2}+2\right)\right]\right. \text {, } \\
& \left.\left.\left[2+4 a \operatorname{RootOf}\left(Z^{2}+2\right)\right],\left[1+2 a \operatorname{RootOf}\left(Z^{2}+2\right)\right]\right]\right] \\
& >\text { LogSreg := SregseptrueBesSq (L, Sreg, ext) [3]; } \\
& \text { LogSreg := [] } \\
& >\text { RemSreg := SregseptrueBesSq (L, Sreg, ext) [2]; } \\
& \text { RemSreg := [] }
\end{aligned}
$$

Since NRemS $_{\text {reg }} \neq[$ ], we know some zeroes of $f$. Those zeroes represent all the zeroes of $f$ because the exponent differences of $\mathrm{L}$ at elements of $\mathrm{NRemS}_{\text {reg }}$ are not in $\mathbb{Q}$.

In order to make our codes faster, we have implemented one procedure called IrrRegAppsingBesSq which gives at the same time and, by avoiding duplicate computations, in a shorter time all the outputs of the procedures irrsingBesSq, regsingtrueBesSq and SregseptrueBesSq.

$>\mathrm{R} 1:=\operatorname{IrrRegAppsingBesSq}(\mathrm{L}, \mathrm{t}, \mathrm{E}, \mathrm{ext})$ :

With our code called BesSqSubst, we can compute the candidates for $f$ up to a constant (the polar part of $f$ ).

$>\mathrm{F} 1:=\operatorname{BesSqSubst}(\mathrm{L}, \mathrm{x}, \mathrm{t}, \mathrm{R} 1[1]$, ext $)$;

$$
F 1:=\left[6(x-1)^{-5}-13(x-1)^{-4}+8(x-1)^{-3}-(x-1)^{-2}\right]
$$

With our code called CandichangvarBesSq, we can compute all the candidates for the change of variables parameter $f$.

$>$ CandichangvarBesSq(F1, R1, ext);

$$
\left[-\frac{(x-2)^{2}(x-7)}{(x-1)^{5}}\right]
$$

Since the exponent differences of $\mathrm{L}$ at elements of $\mathrm{RS}_{\text {reg }}$ are not in $\mathbb{Q}$, we are in the irrational case. Our code called findBesSqIrr gives us the set of candidates for $(\nu, f)$.

$>\quad$ indBesSqIrr $(\mathrm{L}, \mathrm{R} 1, \mathrm{~F} 1, \mathrm{x}, \mathrm{t}, \mathrm{ext})$;

$$
\left[\left[a \operatorname{RootOf}\left({ }_{-} Z^{2}+2\right)+\frac{1}{2}, 1+a \operatorname{RootOf}\left({ }_{-} Z^{2}+2\right)\right],-\frac{(x-2)^{2}(x-7)}{(x-1)^{5}}\right]
$$


All the transformation parameters, if they exist, and also the Bessel square parameter can be given using our code called BesSqSolutions:

$>$ BesSqSolutions(L);

$$
\left\{\left[a \operatorname{RootOf}\left(Z^{2}+2\right)+1 / 2,[0],[1],-\frac{(x-2)^{2}(x-7)}{(-1+x)^{5}}\right]\right\}
$$

Hence, L has Bessel square type solutions with Bessel square parameter $\nu=a$. $\operatorname{RootOf}\left(x^{2}+2\right)+1 / 2$ and transformation parameters: $r=0, r_{0}=1, r_{1}=0, r_{2}=0$ and $f=-\frac{(x-2)^{2}(x-7)}{(x-1)^{5}}$.

\subsection{Change of variable parameters are not squares of rational functions in $k(x)$}

Since $f \in k(x)$, we will assume $f=\frac{\mathrm{A}}{\mathrm{B}}$ with $\mathrm{A}, \mathrm{B} \in k[x], \mathrm{B} \operatorname{monic}$ and $\operatorname{gcd}(\mathrm{A}, \mathrm{B})=1$.

in the whole section, we will consider the situation (4.1).

The problem now is how to get information about A and B from L (our input operator).

\subsubsection{Exponent differences}

The generalized exponents of $\mathrm{L}_{\breve{B}_{\nu}^{2}}$ at 0 and $\infty$ are

$>$ gen_exp $(\mathrm{LBB}, \mathrm{t}, \mathrm{x}=0)$;

$$
[[0, t=x],[-\nu, t=x],[\nu, t=x]]
$$

$>\quad$ gen_exp (LBB, t, $x=$ infinity);

$$
\left[\left[\frac{1}{2}, t=\frac{1}{x}\right],\left[\frac{1}{t}+\frac{1}{2}, t^{2}=\frac{1}{x}\right]\right]
$$

Hence the ramification index of $\mathrm{L}_{\check{B}_{\nu}^{2}}$ is 2 at $x=\infty$ and 1 at all the other points. Using the assumptions of the last chapter, the generalized exponents of $\mathrm{M}$ at

- a zero $p$ of $f$ are

$$
\left[0, \quad-m_{p} \nu, \quad m_{p} \nu\right]
$$

where $m_{p}$ is the multiplicity order of $p$ as a zero of $f$,

- a pole $p$ of $f$ are

$$
\left[\frac{m_{p}}{2}, \quad \frac{m_{p}}{2}+\sum_{j=-m_{p}}^{-1} j \bar{f}_{j+m_{p}} t_{p}^{j / 2}, \quad \frac{m_{p}}{2}-\sum_{j=-m_{p}}^{-1} j \bar{f}_{j+m_{p}} t_{p}^{j / 2}\right]
$$

where $m_{p}$ is the multiplicity order of $p$ as a pole of $f$ and

$$
\left\{\begin{aligned}
f & =t_{p}^{-m_{p}} \sum_{j=0}^{+\infty} f_{j-m_{p}} t_{p}^{j}, \quad \text { with } f_{j-m_{p}} \in k \text { and } f_{-m_{p}} \neq 0 \\
\left(\sum_{j=0}^{+\infty} f_{j-m_{p}} t_{p}^{j}\right)^{1 / 2} & =\sum_{j=0}^{+\infty} \bar{f}_{j} t_{p}^{j / 2} \quad \text { with } \bar{f}_{j} \in k .
\end{aligned}\right.
$$


So the ramification index of $\mathrm{M}$ belongs to $\{1,2\}$ for the poles of $f$ and is 1 for all the other points. Since the exp-product and gauge transformations don't change the ramification index, the ramification index of $\mathrm{L}$ belongs to $\{1,2\}$ for the poles of $f$ and is 1 for all the other points, otherwise L can't have Bessel square root type solutions.

Using (4.24) and (4.25) we have that the exponents differences, modulo a factor -1 , of $\mathrm{M}$ at

- a zero $p$ of $f$ are

$$
\left[-m_{p} \nu, \quad m_{p} \nu, \quad 2 m_{p} \nu\right]
$$

- a pole $p$ of $f$ are

$$
\left[\sum_{j=-m_{p}}^{-1} j \bar{f}_{j+m_{p}} t_{p}^{j / 2}, \quad-\sum_{j=-m_{p}}^{-1} j \bar{f}_{j+m_{p}} t_{p}^{j / 2}, \quad-2 \sum_{j=-m_{p}}^{-1} j \bar{f}_{j+m_{p}} t_{p}^{j / 2}\right]
$$

By using now the fact that the exponent difference is invariant modulo $\frac{1}{n} \mathbb{Z}$ (here $n \in\{1,2\}$ for the poles of $f$ and $n=1$ for all the other points) under the exp-product and gauge transformations, the exponent differences of $\mathrm{L}$ at

- a zero $p$ of $f$ are

$$
\left[-m_{p} \nu+\alpha_{1}, \quad m_{p} \nu+\alpha_{2}, \quad 2 m_{p} \nu+\alpha_{3}\right]
$$

with $\alpha_{1}, \alpha_{2}, \alpha_{3} \in \mathbb{Z}$

- a pole $p$ of $f$ are

$$
\left[\beta_{1}+\sum_{j=-m_{p}}^{-1} j \bar{f}_{j+m_{p}} t_{p}^{j / 2}, \quad \beta_{2}-\sum_{j=-m_{p}}^{-1} j \bar{f}_{j+m_{p}} t_{p}^{j / 2}, \quad \beta_{3}-2 \sum_{j=-m_{p}}^{-1} j \bar{f}_{j+m_{p}} t_{p}^{j / 2}\right]
$$

with $\beta_{1}, \beta_{2}, \beta_{3} \in \frac{1}{2} \mathbb{Z}$.

Since one of the main tool of our work are exponent differences and for the reason of generalization, we will consider the first two exponent differences. The third one will be used as a necessary condition to test if we are solving in terms of Bessel square root functions $\check{\mathrm{B}}_{\nu}^{2}=\mathrm{B}_{\nu}^{2}(\sqrt{x})$.

Definition 4.14. Let us consider the situation in (4.1). We define the exponent differences of $\mathrm{L}$ at

- a zero $p$ of $f$ by

$$
\Delta(\mathrm{L}, p)= \pm m_{p} \nu \bmod \mathbb{Z}
$$

where $m_{p}$ is the multiplicity order of $p$ as a zero of $f$,

- a pole $p$ of $f$ by

$$
\Delta(\mathrm{L}, p)= \pm \sum_{j=-m_{p}}^{-1} j \bar{f}_{j+m_{p}} t_{p}^{j / 2} \bmod \frac{1}{2} \mathbb{Z}
$$


where $m_{p}$ is the multiplicity order of $p$ as a pole of $f$ and

$$
\left\{\begin{aligned}
f & =t_{p}^{-m_{p}} \sum_{j=0}^{+\infty} f_{j-m_{p}} t_{p}^{j}, \quad \text { with } f_{j-m_{p}} \in k \text { and } f_{-m_{p}} \neq 0, \\
\left(\sum_{j=0}^{+\infty} f_{j-m_{p}} t_{p}^{j}\right)^{1 / 2} & =\sum_{j=0}^{+\infty} \bar{f}_{j} t_{p}^{j / 2} \quad \text { with } \bar{f}_{j} \in k .
\end{aligned}\right.
$$

Since we know how to find all the poles of $f$ (using Corollary 3.13) which are elements of the set $\mathrm{S}_{\mathrm{irr}}(\mathrm{L})$, and also their multiplicity orders by using their exponent differences, we can start in order to find $f=\frac{\mathrm{A}}{\mathrm{B}}$, to first find the truncated series for $f$ at all its poles by squaring the truncated series for $f^{1 / 2}$ at all those poles.

\subsubsection{Truncated series for $f$}

This lemma gives us the form of the power of $t_{p}$ in the series expansion of $f^{1 / 2}$ at a point $p$.

Lemma 4.15. Let $p$ be a point in $k$. If $f \in k(x)$ and $f^{1 / 2}=\sum_{i} a_{i} t_{p}^{i}$, where $i \in \frac{1}{2} \mathbb{Z}, a_{i} \in k$ and $t_{p}$ is the local parameter at $x=p$, then the set $\left\{i \mid a_{i} \neq 0\right\}$ is either a subset of $\mathbb{Z}$ or a subset of $\frac{1}{2}+\mathbb{Z}$.

Proof. Similar to the proof of Lemma 9 in [42].

Definition 4.16. Let $p \in k$ and $g=\sum_{i=N}^{\infty} a_{i} t_{p}^{i / 2}, a_{i} \in k$ and $a_{N} \neq 0$. We say that we have an $n$-term truncated power series for $f$ when the coefficients of $t_{p}^{N / 2}, \ldots, t_{p}^{(N+n-1) / 2}$ are known.

Remark 4.17. If an $n$-term truncated series for $f^{1 / 2}$ is known then we can compute a $n$-term truncated series for $f$.

Let us have a look on the exponent difference of $\mathrm{L}$ at a pole $p$ of $f$ in (4.31). It can be brought in the form

$$
\beta+\sum_{j=-m_{p}}^{-1} a_{j} t_{p}^{j / 2}, \text { with } \beta \in \mathbb{Z}
$$

where $a_{j}= \pm j \bar{f}_{j+m_{p}}$ and

$$
\left\{\begin{aligned}
f & =t_{p}^{-m_{p}} \sum_{j=0}^{+\infty} f_{j-m_{p}} t_{p}^{j}, \quad \text { with } f_{j-m_{p}} \in k \text { and } f_{-m_{p}} \neq 0 \\
\left(\sum_{j=0}^{+\infty} f_{j-m_{p}} t_{p}^{j}\right)^{1 / 2} & =\sum_{j=0}^{+\infty} \bar{f}_{j} t_{p}^{j / 2} \quad \text { with } \bar{f}_{j} \in k
\end{aligned}\right.
$$

So a truncated series for $f^{1 / 2}$, modulo a factor -1 , at a pole $p$ is

$$
\sum_{j=-m_{p}}^{-1} \frac{a_{j}}{j} t_{p}^{j / 2}
$$


Let $p \in \mathrm{S}_{\mathrm{irr}}(\mathrm{L})$ and $f^{1 / 2}=\sum_{i=-m_{p}}^{\infty} a_{i} t_{p}^{i / 2}, a_{i} \in k$ with $m_{p}$ the multiplicity order of $p$ as a zero of $f$. By (4.32), we have a truncated series for $f^{1 / 2}$ with $\left\lceil m_{p} / 2\right\rceil$ terms (see [42]). We square it to obtain a truncated series of $f$ at $p$. But this truncated series for $f$ has $\left\lceil m_{p} / 2\right\rceil$ terms (see Remark 4.17) which is only half (rounded up) of the polar part of $f$.

We have implemented in Maple an algorithm called SirrBesSqRootinfo1 to find all those truncated series for $f$ related to the elements of $\mathrm{S}_{\text {irr }}(\mathrm{L})$.

Since we know how to find the truncated series for $f$, the next step will be to find, using the poles of $f$, the denominator $\mathrm{B}$ of $f$.

\subsubsection{How to compute the denominator B of $f$}

We retrieve B from $\mathrm{S}_{\text {irr }}(\mathrm{L})$ as follows:

Lemma 4.18. Consider the situation (4.1) and $f=\mathrm{A} / \mathrm{B}$ with $\mathrm{A}, \mathrm{B} \in k[x], \mathrm{B}$ monic and $\operatorname{gcd}(\mathrm{A}, \mathrm{B})=1$. Then

$$
\mathrm{B}=\prod_{p \in \mathrm{S}_{i r r}(\mathrm{~L})}(x-p)^{m_{p}}=\prod_{p \in \mathrm{S}_{i r r}(\mathrm{~L})} t_{p}^{m_{p}}=\prod_{P_{s} \in \overline{\mathrm{S}}_{i r r}(\mathrm{~L}) \backslash\{1\}} P_{s}^{m_{s}}
$$

where $\forall p \in \mathrm{S}_{\text {irr }}(\mathrm{L}), f=\sum_{i=-m_{p}}^{\infty} a_{i} t_{p}^{i}$ ( $m_{p}$ is the multiplicity order of $p$ as a pole of $\left.f\right)$.

Proof. We We just use, by Corollary 3.13, the fact that $\mathrm{S}_{\mathrm{irr}}(\mathrm{L})$ represents all the poles of $f$.

The algorithm SirrBesSqRootinfo1 that we have implemented computes this denominator $\mathrm{B}$ of $f$.

We know how to find B (denominator of $f$ ). The next problem is now to find the numerator $\mathrm{A}$ of $f$. In order to solve it, we will need to find a bound for the degree of A.

\subsubsection{How to get a bound for the degree of the numerator A of $f$}

Remarks 4.19. (i) If $\infty \in \mathrm{S}_{\text {irr }}(\mathrm{L})$ we will have $\operatorname{deg}(\mathrm{A})>\operatorname{deg}(\mathrm{B})$.

(ii) If $\infty \in \mathrm{S}_{\text {reg }}(\mathrm{L})$ we will have $\operatorname{deg}(\mathrm{A})<\operatorname{deg}(\mathrm{B})$.

(iii) If $\infty$ is an apparent singularity of $\mathrm{L}, \infty$ can be a zero of $f$ but never a pole of $f$. So $\operatorname{deg}(\mathrm{A}) \leq \operatorname{deg}(\mathrm{B}) \quad($ if $\infty$ is not a zero of $f$ then $\operatorname{deg}(\mathrm{A})=\operatorname{deg}(\mathrm{B}))$.

The following lemma gives us a bound for the degree of A.

Lemma 4.20. Let

$$
d_{\mathrm{A}}= \begin{cases}\operatorname{deg}(\mathrm{B})+m_{\infty} & \text { if } \infty \in \mathrm{S}_{i r r}(\mathrm{~L}) \\ \operatorname{deg}(\mathrm{B}) & \text { otherwise }\end{cases}
$$

where $m_{\infty}$ is the multiplicity order of $\infty$ as a pole of $f\left(\infty \in \mathrm{S}_{\text {irr }}(\mathrm{L})\right)$.

(i) If $\infty \in \mathrm{S}_{\text {irr }}(\mathrm{L})$ then $\operatorname{deg}(\mathrm{A})=d_{\mathrm{A}}$,

(ii) if $\infty \in \mathrm{S}_{\text {reg }}(\mathrm{L})$ then $\operatorname{deg}(\mathrm{A})<d_{\mathrm{A}}$, 
(iii) otherwise $\operatorname{deg}(\mathrm{A}) \leq d_{\mathrm{A}}\left(\infty \notin \mathrm{S}_{\text {irr }}(\mathrm{L}) \cup \mathrm{S}_{\text {reg }}(\mathrm{L})\right)$.

Proof. It follows from Remarks 4.19.

Hence, $d_{\mathrm{A}}$ given by (4.34) is a bound for the degree of the numerator $\mathrm{A}$ of $f$.

Corollary 4.21. For $s \in \mathrm{S}_{\text {irr }}(\mathrm{L})$, let $m_{s}$ be its multiplicity order as a pole of $f$. Then

$$
d_{\mathrm{A}}=\sum_{s \in \mathrm{S}_{i r r}(\mathrm{~L})} m_{s}
$$

Proof. By Lemma 4.20 we have

$$
d_{\mathrm{A}}=\left\{\begin{array}{ll}
\operatorname{deg}(\mathrm{B})+m_{\infty} & \text { if } \infty \in \mathrm{S}_{\mathrm{irr}}(\mathrm{L}), \\
\operatorname{deg}(\mathrm{B}) & \text { otherwise }
\end{array} \Rightarrow d_{\mathrm{A}}= \begin{cases}\sum_{s \in \mathrm{S}_{\mathrm{irr}}(\mathrm{L}) \backslash\{\infty\}} m_{s}+m_{\infty} & \text { if } \infty \in \mathrm{S}_{\mathrm{irr}}(\mathrm{L}), \\
\sum_{s \in \mathrm{S}_{\mathrm{irr}}(\mathrm{L})} m_{s} & \text { otherwise }\end{cases}\right.
$$

So $d_{\mathrm{A}}=\sum_{s \in \mathrm{S}_{\mathrm{irr}}(\mathrm{L})} m_{s}$.

The algorithm SirrBesSqRootinfo1 that we have implemented computes $d_{\mathrm{A}}$.

Now we know a bound $d_{\mathrm{A}}$ for the degree of $\mathrm{A}$. The next step will be to find its coefficients. In order to achieve it, we will first see how to get linear equations for those coefficients of $\mathrm{A}$.

\subsubsection{How to get linear equations for the coefficients of the nu- merator A of $f$}

Since we know a bound for the degree of A, denoted by $d_{\mathrm{A}}$, we can write

$$
\mathrm{A}=\sum_{i=0}^{d_{\mathrm{A}}} a_{i} x^{i}, \quad a_{i} \in k .
$$

So we have $d_{\mathrm{A}}+1$ unknowns: $a_{0}, \ldots, a_{d_{\mathrm{A}}}$. The equations for those coefficients of $\mathrm{A}$ will come from the set $\mathrm{S}_{\text {irr }}(\mathrm{L}) \cup \mathrm{S}_{\text {reg }}(\mathrm{L})$. For a point $s \in \mathrm{S}_{\text {irr }}(\mathrm{L}) \cup \mathrm{S}_{\text {reg }}(\mathrm{L})$, we will deal with two cases:

- when $s \in k$,

- when $s \in \bar{k}$ but $s \notin k . \bar{k}$ is the algebraic closure of $k$.

That is done by taking instead of $x-s$ the minimal polynomial $P_{s}$ of $s$ over $k$ and working on $k(s)$ instead of $k$.

Lemma 4.22. Let us assume $\mathrm{S}_{r e g}(\mathrm{~L}) \neq \emptyset$. Then the remainder of the Euclidean division of $\mathrm{A}$ by $\prod_{P_{s} \in \overline{\mathrm{S}_{\text {reg }}(\mathrm{L})}} P_{s}$ will give us $\sum_{P_{s} \in \overline{\mathrm{S}_{\text {reg }}(\mathrm{L})}} \operatorname{deg}\left(P_{s}\right)$ linear equations for the coefficients of $\mathrm{A}$.

Proof. For $s \in \mathrm{S}_{\text {reg }}(\mathrm{L})$, let $m_{s}$ be its multiplicity order as a zero of $f$. We can write $\mathrm{A}$ in the form

$$
\mathrm{A}=c \prod_{P_{s} \in \overline{\mathrm{S}_{\mathrm{reg}}(\mathrm{L})}} P_{s}^{m_{s}} \text { with } c \in k
$$


Since $m_{s} \geq 1 \forall s \in \mathrm{S}_{\mathrm{reg}}(\mathrm{L})$, let $\mathrm{R}$ be the remainder of $\mathrm{A}$ divided by $\prod_{P_{s} \in \overline{\mathrm{S}_{\mathrm{reg}}(\mathrm{L})}} P_{s}$. Hence $\mathrm{R}$ is a polynomial of degree $-1+\sum_{P_{s} \in \overline{\mathrm{S}_{\text {reg }}(\mathrm{L})}} \operatorname{deg}\left(P_{s}\right)$. The fact that $m_{s} \in \mathbb{N} \backslash\{0\} \forall s \in$ $\mathrm{S}_{\text {reg }}(\mathrm{L})$ implies $\mathrm{R}=0$ and therefore we will have $\sum_{P_{s} \in \overline{\mathrm{S}_{\text {reg }}(\mathrm{L})}} \operatorname{deg}\left(P_{s}\right)$ linear equations for the coefficients of A.

Remark 4.23. By the proof of Lemma $4.22, \infty \in \mathrm{S}_{\text {reg }}(\mathrm{L})$ gives us one equation: $a_{d_{\mathrm{A}}}=0$. That means we have $\operatorname{deg}(\mathrm{A})<d_{\mathrm{A}}$.

Lemma 4.24. For $s \in \mathrm{S}_{\text {irr }}(\mathrm{L})$ let $m_{s}$ be its multiplicity order as a zero of $f, f_{s}$ the polar part of $f$ at $s$ and $\bar{f}_{s}$ the $\left\lceil m_{s} / 2\right\rceil$-truncated series of $f$ at $s$. Let

$$
u(x)=\left[f-\sum_{P_{s} \in \overline{\mathrm{S}_{i r r}(\mathrm{~L})}} \bar{f}_{s}\right] \cdot \prod_{P_{s} \in \overline{\mathrm{S}_{i r r}(\mathrm{~L})} \backslash\{1\}} P_{s}^{\left\lfloor m_{s} / 2\right\rfloor} .
$$

Then $u(x) \in k[x]$ and the remainder of the Euclidean division of numer $(u(x))$ by $\operatorname{denom}(u(x))$ will give us $\sum_{P_{s} \in \overline{\mathrm{S}_{i r r}(\mathrm{~L}) \backslash\{1\}}} \operatorname{deg}\left(P_{s}\right) \cdot\left\lceil m_{s} / 2\right\rceil$ linear equations for the coefficients of $\mathrm{A}$.

Furthermore, if $\infty \in \mathrm{S}_{\text {irr }}(\mathrm{L})$, the quotient of the Euclidean division of numer $(u(x))$ by $\operatorname{denom}(u(x))$ will give us, in addition, $\left\lceil m_{\infty} / 2\right\rceil$ linear equations.

Proof. For $s \in \mathrm{S}_{\text {irr }}(\mathrm{L})$ let $m_{s}$ be its multiplicity order as a zero of $f, f_{s}$ the polar part of $f$ at $s$ and $\bar{f}_{s}$ the $\left\lceil m_{s} / 2\right\rceil$-truncated series of $f$ at $s$. We have

$$
f_{s}=\bar{f}_{s}+\widetilde{f}_{s} \text { where } \widetilde{f}_{s}= \begin{cases}\sum_{i=m_{s}}^{1} b_{i} x^{\lfloor i / 2\rfloor} & b_{i} \in k \quad \text { if } s=\infty, \\ \sum_{i=m_{s}}^{1} b_{i} t_{s}^{-\lfloor i / 2\rfloor} & b_{i} \in k \text { otherwise. }\end{cases}
$$

By our algorithm SirrBesSqRootinfo1 we can compute all the $\bar{f}_{s}$ for $s \in \mathrm{S}_{\text {irr }}(\mathrm{L})$.

$$
f-\sum_{P_{s} \in \overline{\mathrm{S}_{\mathrm{irr}}}(\mathrm{L})} \bar{f}_{s}=f-\sum_{P_{s} \in \overline{\mathrm{S}_{\mathrm{irr}}(\mathrm{L})}}\left(f_{s}-\tilde{f}_{s}\right)=\left(f-\sum_{P_{s} \in \overline{\mathrm{S}_{\mathrm{irr}}(\mathrm{L})}} f_{s}\right)+\widetilde{f}_{\infty}+\sum_{P_{s} \in \overline{\mathrm{S}_{\mathrm{irr}}(\mathrm{L})} \backslash\{1\}} \tilde{f}_{s}
$$

where $\tilde{f}_{\infty} \in k[x]$ and $\widetilde{f}_{\infty}=0$ if $\infty \notin \mathrm{S}_{\text {irr }}(\mathrm{L})$. Since $f-\sum_{P_{s} \in \overline{\mathrm{S}_{\text {irr }}(\mathrm{L})}} f_{s}=a \in k$, we get

$$
f-\sum_{P_{s} \in \overline{\mathrm{S}_{\mathrm{irr}}(\mathrm{L})}} \bar{f}_{s}=a+\tilde{f}_{\infty}+\sum_{P_{s} \in \overline{\mathrm{S}}_{\mathrm{irr}}(\mathrm{L}) \backslash\{1\}} \tilde{f}_{s} .
$$

$f \in k(x)$ implies that the polar part of $f$ at $s$ doesn't have half integer powers (element of $1 / 2+\mathbb{Z})$ for all $s \in \mathrm{S}_{\text {irr }}(\mathrm{L})$. Hence $\forall s \in \mathrm{S}_{\text {irr }}(\mathrm{L}), \widetilde{f}_{s} \in k(x), \bar{f}_{s} \in k(x), \tilde{f}_{\infty} \in k[x]$ and $\bar{f}_{\infty} \in k[x]$. So we have

$$
\left\{\begin{array}{l}
a+\widetilde{f}_{\infty} \in k[x], \\
\sum_{P_{s} \in \mathrm{S}_{\mathrm{irr}}(\mathrm{L}) \backslash\{1\}} \widetilde{f}_{s}=\frac{h_{1}(x)}{\prod_{P_{s} \in \overline{\mathrm{S}_{\mathrm{irr}}(\mathrm{L})} \backslash\{1\}} P_{s}^{\left\lfloor m_{s} / 2\right\rfloor}}, h_{1}(x) \in k[x], \\
\sum_{P_{s} \in \overline{\mathrm{S}_{\mathrm{irr}}(\mathrm{L})}} \bar{f}_{s}=\bar{f}_{\infty}+\frac{\prod_{2}(x)}{\prod_{P_{s} \in \overline{\mathrm{S}_{\mathrm{irr}}(\mathrm{L})} \backslash\{1\}} P_{s}^{m_{s}}}, h_{2}(x) \in k[x]
\end{array}\right.
$$


with $\operatorname{deg}\left(h_{1}\right)<\sum_{P_{s} \in \mathrm{S}_{\mathrm{irr}}(\mathrm{L}) \backslash\{1\}} \operatorname{deg}\left(P_{s}\right) \cdot\left\lfloor m_{s} / 2\right\rfloor$ and $\operatorname{deg}\left(h_{2}\right)<\sum_{P_{s} \in \sum_{\mathrm{Sirr}}(\mathrm{L}) \backslash\{1\}} \operatorname{deg}\left(P_{s}\right) \cdot m_{s}$.

Therefore $f-\sum_{P_{s} \in \overline{\mathrm{S}_{\mathrm{irr}}(\mathrm{L})}} \bar{f}_{s}=a+\tilde{f}_{\infty}+\frac{h_{1}(x)}{\prod_{P_{s} \in \overline{\mathrm{S}_{\mathrm{irr}}(\mathrm{L})} \backslash\{1\}} P_{s}^{\left\lfloor m_{s} / 2\right\rfloor}}$. Now $u(x)$ becomes

$$
\begin{aligned}
u(x)= & {\left[f-\sum_{P_{s} \in \overline{\mathrm{S}_{\text {irr }}(\mathrm{L})}} \bar{f}_{s}\right] \cdot \prod_{P_{s} \in \overline{\mathrm{S}_{\mathrm{irr}}(\mathrm{L}) \backslash\{1\}}} P_{s}^{\left\lfloor m_{s} / 2\right\rfloor} } \\
= & {\left[a+\widetilde{f}_{\infty}+\frac{h_{1}(x)}{\prod_{P_{s} \in \overline{\mathrm{S}_{\text {irr }}(\mathrm{L})} \backslash\{1\}} P_{s}^{\left\lfloor m_{s} / 2\right\rfloor}}\right] \cdot \prod_{P_{s} \in \overline{\mathrm{Sirr}}(\mathrm{L}) \backslash\{1\}} P_{s}^{\left\lfloor m_{s} / 2\right\rfloor} } \\
= & \left(a+\widetilde{f}_{\infty}\right) \cdot \prod_{P_{s} \in \overline{\mathrm{S}_{\mathrm{irr}}(\mathrm{L})} \backslash\{1\}} P_{s}^{\left\lfloor m_{s} / 2\right\rfloor}+h_{1}(x) \\
\Rightarrow & u(x) \in k[x] \text { and } \operatorname{deg}(u(x))=\left\lfloor m_{\infty} / 2\right\rfloor+\sum_{P_{s} \in \overline{\mathrm{S}_{\mathrm{irr}}(\mathrm{L}) \backslash\{1\}}} \operatorname{deg}\left(P_{s}\right) \cdot\left\lfloor m_{s} / 2\right\rfloor .
\end{aligned}
$$

On the other hand, since $f=\mathrm{A} / \mathrm{B}$,

$$
\begin{aligned}
& u(x)=\left[\frac{\mathrm{A}}{\mathrm{B}}-\sum_{P_{s} \in \overline{\mathrm{S}_{\mathrm{irr}}(\mathrm{L})}} \bar{f}_{s}\right] \cdot \prod_{P_{s} \in \overline{\mathrm{S}_{\mathrm{irr}}(\mathrm{L})} \backslash\{1\}} P_{s}^{\left\lfloor m_{s} / 2\right\rfloor} \\
& =\left[\frac{\sum_{i=0}^{d_{\mathrm{A}}} a_{i} x^{i}}{\prod_{P_{s} \in \overline{\mathrm{S}_{\mathrm{irr}}(\mathrm{L})} \backslash\{1\}} P_{s}^{m_{s}}}-\bar{f}_{\infty}-\frac{h_{2}(x)}{\prod_{P_{s} \in \mathrm{S}_{\mathrm{irr}}(\mathrm{L}) \backslash\{1\}} P_{s}^{m_{s}}}\right] \cdot \prod_{P_{s} \in \overline{\mathrm{S}_{\mathrm{irr}}(\mathrm{L}) \backslash\{1\}}} P_{s}^{\left\lfloor m_{s} / 2\right\rfloor} \\
& \text { using (4.35), (4.37) and Lemma } 4.18 \\
& =\frac{1}{\prod_{P_{s} \in \overline{\mathrm{S}_{\mathrm{irr}}(\mathrm{L}) \backslash\{1\}}} P_{s}^{m_{s}-\left\lfloor m_{s} / 2\right\rfloor}}\left[\sum_{i=0}^{d_{\mathrm{A}}} a_{i} x^{i}-\bar{f}_{\infty} \cdot \prod_{P_{s} \in \overline{\mathrm{S}_{\mathrm{irr}}(\mathrm{L}) \backslash\{1\}}} P_{s}^{m_{s}}-h_{2}(x)\right] \\
& =\frac{1}{\prod_{P_{s} \in \overline{\mathrm{S}_{\mathrm{irr}}(\mathrm{L}) \backslash\{1\}}} P_{s}^{\left\lceil m_{s} / 2\right\rceil}}\left[\sum_{i=0}^{d_{\mathrm{A}}} a_{i} x^{i}-\bar{f}_{\infty} \cdot \prod_{P_{s} \in \overline{\mathrm{S}_{\mathrm{irr}}(\mathrm{L}) \backslash\{1\}}} P_{s}^{m_{s}}-h_{2}(x)\right] .
\end{aligned}
$$

Since $\sum_{i=0}^{d_{\mathrm{A}}} a_{i} x^{i}-\bar{f}_{\infty} \cdot \prod_{P_{s} \in \overline{\mathrm{S}_{\mathrm{irr}}(\mathrm{L})} \backslash\{1\}} P_{s}^{m_{s}}-h_{2}(x) \in k[x]$ and we don't know the coefficients $a_{i}$ of $\mathrm{A}$,

$$
\left\{\begin{array}{l}
\operatorname{numer}(u(x))=\sum_{i=0}^{d_{\mathrm{A}}} a_{i} x^{i}-\bar{f}_{\infty} \cdot \prod_{P_{s} \in \overline{\mathrm{S}_{\mathrm{irr}}(\mathrm{L})} \backslash\{1\}} P_{s}^{m_{s}}-h_{2}(x), \\
\operatorname{denom}(u(x))=\sum_{P_{s} \in \overline{\mathrm{S}_{\mathrm{irr}}(\mathrm{L}) \backslash\{1\}}} P_{s}^{\left\lceil m_{s} / 2\right\rceil} .
\end{array}\right.
$$

Let $\mathrm{R}$ be the remainder of numer $(u(x))$ divided by denom $(u(x))$. Hence $\mathrm{R}$ is a polynomial of degree $-1+\sum_{P_{s} \in \overline{\mathrm{S}_{\mathrm{irr}}(\mathrm{L}) \backslash\{1\}}} \operatorname{deg}\left(P_{s}\right) \cdot\left\lceil m_{s} / 2\right\rceil$. The fact that $u(x) \in k[x]$ implies $\mathrm{R}=0$ and 
therefore we will have $\sum_{P_{s} \in \overline{\mathrm{S}_{\text {irr }}(\mathrm{L}) \backslash\{1\}}} \operatorname{deg}\left(P_{s}\right) \cdot\left\lceil m_{s} / 2\right\rceil$ linear equations for the coefficients of A.

Let us assume that $\infty \in \mathrm{S}_{\text {irr }}(\mathrm{L})$. Let $\mathrm{Q}$ be the quotient of numer $(u(x))$ divided by $\operatorname{denom}(u(x))$ and $d_{1}$ its degree. Then $d_{1}=\operatorname{deg}(\mathrm{Q})=\operatorname{deg}(u(x))$ since $u(x) \in k[x]$. Hence, by (4.38) we have $d_{1}=\left\lfloor m_{\infty} / 2\right\rfloor+\sum_{P_{s} \in \overline{\mathrm{S}_{\text {irr }}(\mathrm{L}) \backslash\{1\}}} \operatorname{deg}\left(P_{s}\right) \cdot\left\lfloor m_{s} / 2\right\rfloor$ and this implies

$$
\sum_{P_{s} \in \overline{\mathrm{Sirr}}(\mathrm{L}) \backslash\{1\}} \operatorname{deg}\left(P_{s}\right) \cdot\left\lfloor m_{s} / 2\right\rfloor=d_{1}-\left\lfloor m_{\infty} / 2\right\rfloor .
$$

For $\infty \in \mathrm{S}_{\text {irr }}(\mathrm{L}), u(x)$ will be taken as $u(x) \cdot(1 / x)^{\left\lfloor m_{\infty} / 2\right\rfloor}$ and therefore

$$
\operatorname{deg}(u(x))=\sum_{P_{s} \in \overline{\mathrm{S}_{\mathrm{irr}}(\mathrm{L})} \backslash\{1\}} \operatorname{deg}\left(P_{s}\right) \cdot\left\lfloor m_{s} / 2\right\rfloor .
$$

Since $\operatorname{deg}(\mathrm{Q})=\operatorname{deg}(u(x))$, we will also have $\operatorname{deg}(\mathrm{Q})=\sum_{P_{s} \in \overline{\mathrm{S}_{\mathrm{irr}}(\mathrm{L}) \backslash\{1\}}} \operatorname{deg}\left(P_{s}\right) \cdot\left\lfloor m_{s} / 2\right\rfloor$. So all the coefficients of $\mathrm{Q}$ with degree $i=\sum_{P_{s} \in \overline{\mathrm{S}_{\text {irr }}(\mathrm{L})} \backslash\{1\}} \operatorname{deg}\left(P_{s}\right) \cdot\left\lfloor m_{s} / 2\right\rfloor+1 \cdots d_{1}$ have to be zero. By using (4.40), all the coefficients of Q with degree $i=d_{1}-\left\lfloor m_{\infty} / 2\right\rfloor+1 \cdots d_{1}$ have to be zero. That gives us $d_{1}-\left[d_{1}-\left\lfloor m_{\infty} / 2\right\rfloor+1\right]+1$ linear equations for the coefficients of A. Therefore, we will have $\left\lfloor m_{\infty} / 2\right\rfloor$ linear equations for the coefficients of $\mathrm{A}$ when $\infty \in \mathrm{S}_{\text {irr }}(\mathrm{L})$.

Remarks 4.25. $\quad$ a- By Lemma 4.22 and Lemma 4.24, we have

$$
\sum_{P_{s} \in \overline{\mathrm{S}_{\text {reg }}(\mathrm{L})}} \operatorname{deg}\left(P_{s}\right)+\sum_{P_{s} \in \overline{\mathrm{S}_{\text {irr }}(\mathrm{L})}} \operatorname{deg}\left(P_{s}\right) \cdot\left\lceil m_{s} / 2\right\rceil \text { linear equations for the coefficients of } \mathrm{A}
$$

where $m_{s}$ is the multiplicity order of $s \in \mathrm{S}_{\text {irr }}(\mathrm{L})$ as a pole of $f$. That means we have

$$
\sum_{s \in \mathrm{S}_{r e g}(\mathrm{~L})} 1+\sum_{s \in \mathrm{S}_{i r r}(\mathrm{~L})}\left\lceil m_{s} / 2\right\rceil \text { linear equations. }
$$

b- Since $\mathrm{S}_{\text {reg }}(\mathrm{L})$ can be an empty set but $\mathrm{S}_{\text {irr }}(\mathrm{L})$ never, we have at least

$$
\sum_{P_{s} \in \overline{\mathrm{S}_{i r r}(\mathrm{~L})}} \operatorname{deg}\left(P_{s}\right) \cdot\left\lceil m_{s} / 2\right\rceil \text { linear equations for the coefficients of } \mathrm{A} \text {. }
$$

That means

$$
\sum_{s \in \mathrm{S}_{\text {irr }}(\mathrm{L})}\left\lceil m_{s} / 2\right\rceil \text { linear equations. }
$$

Lemma 4.26. The number of linear equations for the coefficients of $\mathrm{A}$ is greater or equal to $\frac{1}{2} d_{\mathrm{A}}+\sum_{p \in \mathrm{S}_{\text {reg }}(\mathrm{L})} 1$.

Proof. By Corollary 4.21 we have

$$
d_{\mathrm{A}}=\sum_{s \in \mathrm{S}_{\mathrm{irr}}(\mathrm{L})} m_{s} \Rightarrow \frac{1}{2} d_{\mathrm{A}}=\sum_{s \in \mathrm{S}_{\mathrm{irr}}(\mathrm{L})} \frac{m_{s}}{2} \leq \sum_{s \in \mathrm{S}_{\mathrm{irr}}(\mathrm{L})}\left\lceil\frac{m_{s}}{2}\right\rceil .
$$


Hence, by the part (a) of Remarks 4.25, the number of linear equations for the coefficients of $\mathrm{A}$ is greater or equal to $\frac{1}{2} d_{\mathrm{A}}+\sum_{p \in \mathrm{S}_{\mathrm{reg}}(\mathrm{L})} 1$.

The algorithm SirrBesSqRootinfo1 that we have implemented computes $d_{\mathrm{A}}$ and the number of linear equations satisfied by the coefficients of A.

We have now the linear equations satisfied by the coefficients of the numerator A of $f$. Let $n$ be the number of those equations. If this number is greater than the degree of $\mathrm{A}$ then we can solve those equations to get the coefficients of $\mathrm{A}$. If this is not the case, we will discuss further methods later using the zeroes of $f$ (elements of $\mathrm{S}_{\mathrm{reg}}(\mathrm{L})$ ) and the exponent differences of L at those zeroes. At the same time, we can see how to find candidates for the Bessel parameter $\nu$.

\subsubsection{How to compute the numerator A of $f$ and the Bessel pa- rameter $\nu$}

By comparing $d_{\mathrm{A}}$ and the number $n$ of linear equations satisfied by the coefficients of $\mathrm{A}$ (we can use the algorithm SirrBesSqRootinfo1 to get $n$ ) we have:

1. if $n>d_{\mathrm{A}}$ then we can solve those equations and get A: that is the "Easy case".

2. if $n \leq d_{\mathrm{A}}$, we have to distinguish between two cases:

(a) when all the zeroes of $f$ are known:

i. "Logarithmic case" $(\nu \in \mathbb{Z})$,

ii. "Irrational case" $(\nu \in k \backslash \mathbb{Q})$,

(b) when we are not sure that we know all the zeroes of $f$ : "Rational case" $(\nu \in$ $\mathbb{Q} \backslash \mathbb{Z})$.

Normally, we just have three cases: "Logarithmic case", "Irrational case" and "Rational case". The "Easy case" just helps us to find candidates for $f$. To find candidates for $\nu$ we have to search whether we are in the "Logarithmic case", "Irrational case" or "Rational case" and use a particular technique, too.

\section{Easy Case}

Lemma 4.27. In the Easy case, $\mathrm{S}_{r e g}(\mathrm{~L}) \neq \emptyset$.

Proof. For $s \in \mathrm{S}_{\mathrm{irr}}(\mathrm{L})$, let $m_{s}$ be its multiplicity order as a pole of $f$.

we have

Since $\left\lceil\frac{m_{s}}{2}\right\rceil \leq m_{s} \forall s \in \mathrm{S}_{\text {irr }}(\mathrm{L})$, we get $\sum_{s \in \mathrm{S}_{\mathrm{irr}}(\mathrm{L})}\left\lceil\frac{m_{s}}{2}\right\rceil \leq \sum_{s \in \mathrm{S}_{\mathrm{irr}}(\mathrm{L})} m_{s}$, and by Corollary 4.21

$$
\sum_{s \in \mathrm{S}_{\mathrm{irr}}(\mathrm{L})}\left\lceil\frac{m_{s}}{2}\right\rceil \leq d_{\mathrm{A}} \text {. }
$$

If $\mathrm{S}_{\text {reg }}(\mathrm{L})=\emptyset$ then, using the part $(a)$ of Remarks 4.25 , we will have $n=\sum_{s \in \mathrm{S}_{\text {irr }}(\mathrm{L})}\left\lceil\frac{m_{s}}{2}\right\rceil$ linear equations for the coefficients of A. So by (4.41), $n \leq d_{\mathrm{A}}$, and that means we are not in the Easy case $\left(n>d_{\mathrm{A}}\right)$.

To find candidates for $(\nu, f)$ we proceed as follows: 
1. We solve our linear equations for the coefficients of A using Lemma 4.22 and Lemma 4.24:

(a) If we find solutions then we already have A and therefore $f=\mathrm{A} / \mathrm{B}$ because we know B.

(b) If we don't find solutions then we cannot find Bessel square root $\left(\check{\mathrm{B}}_{\nu}^{2}=\mathrm{B}_{\nu}^{2}(\sqrt{x})\right)$ type solutions for $\mathrm{L}$ using the Easy case. That means the number of linear equations for the coefficients $a_{i}$ of $\mathrm{A}$ is less than or equal to $d_{\mathrm{A}}$.

2. To find candidates for $\nu$ we have to search whether we are in the "Logarithmic case", "Irrational case" or "Rational case" and use a particular technique (those cases will be treated and explained in the next sections). Since $S_{\text {reg }}(L) \neq \emptyset$, we take the first element $s$ in $\mathrm{S}_{\text {reg }}(\mathrm{L})$ and proceed as follows:

(a) if L has a logarithmic solution at $s$ then we are in the logarithmic case and we use its technique.

(b) if L doesn't have a logarithmic solution at $s$ then:

i. if $\Delta(\mathrm{L}, s) \in \mathbb{Q}$, we are in the rational case and we use its technique.

ii. if $\Delta(\mathrm{L}, s) \notin \mathbb{Q}$, we are in the irrational case and we use its technique.

We have implemented in Maple an algorithm called easyBesSqRootf to find candidates for $f$ and another algorithm called easyBesSqRoot to find both candidates for $(\nu, f)$, if they exist. An example for this case can be found in our Maple worksheet associated to this thesis.

\section{Logarithmic case}

As in the Bessel square root case with $f=g^{2}$ and $g \in k(x)$, we know all the zeroes of $f$ : the set $\mathrm{S}_{\mathrm{reg}}(\mathrm{L})$. They have logarithmic solutions and $\nu \in \mathbb{Z}$. So we have now to do a combinatorial search to find their multiplicities as zeroes of A: try all possible combinations of multiplicities of zeroes of A. For a zero $s$ of $f$, let $m_{s}$ be its multiplicity order. We will have

$$
\operatorname{deg}(\mathrm{A})=\sum_{P_{s} \in \overline{\mathrm{S}_{\mathrm{reg}}(\mathrm{L})}} \operatorname{deg}\left(P_{s}\right) \cdot m_{s}
$$

To find the list of combinations of multiplicities $m_{s}$ of $s \in \mathrm{S}_{\mathrm{reg}}(\mathrm{L})$ as zeroes of $\mathrm{A}$ we proceed as follows:

1. We take one element of $\mathrm{S}_{\mathrm{reg}}(\mathrm{L})$ and we call it $s_{0}$.

2. We put $\operatorname{deg}(\mathrm{A})$ in the form

$$
\operatorname{deg}(\mathrm{A})=Q_{s_{0}} \cdot \operatorname{deg}\left(P_{s_{0}}\right)+R_{s_{0}} \text { with } Q_{s_{0}}, R_{s_{0}} \in \mathbb{N} \text { and } 0 \leq R_{s_{0}}<\operatorname{deg}\left(P_{s_{0}}\right) .
$$

By using (4.42) and the fact that $m_{s} \geq 1 \forall s \in \mathrm{S}_{\mathrm{reg}}(\mathrm{L})$, we have $m_{s_{0}} \in\left\{1, \ldots, Q_{s_{0}}\right\}$.

3. For $m_{s_{0}} \in\left\{1, \ldots, Q_{s_{0}}\right\}$, we repeat the process by considering $\overline{\mathrm{S}_{\mathrm{reg}}(\mathrm{L})}=\overline{\mathrm{S}_{\mathrm{reg}}(\mathrm{L})} \backslash\left\{P_{s_{0}}\right\}$ and $\operatorname{deg}(\mathrm{A})=\operatorname{deg}(\mathrm{A})-\operatorname{deg}\left(P_{s_{0}}\right) \cdot m_{s_{0}}$. 
4. At the end, we will have the list of combinations of multiplicities $m_{s}$ of $s \in \mathrm{S}_{\text {reg }}(\mathrm{L})$. The only unknown will be the leading coefficient of A. By Remarks 4.25 we have enough equations to find it.

We have implemented in Maple an algorithm called SearchKnLog to find candidates for A up to a multiplicative constant.

Once we get candidates for $f=\mathrm{A} / \mathrm{B}$, we use the same technique as in the logarithmic case with $f=g^{2}$ and $g \in k(x)$ to find candidates for $\nu$ related to any $f$.

We have also implemented in Maple an algorithm called findBesSqRootln which gives us candidates for $(\nu, f)$, if they exist. An example for this case can be found in our Maple worksheet associated to this thesis.

\section{Irrational case}

Lemma 4.28. In the irrational case, we know all zeroes of $f$ and their multiplicity as well.

Proof.

1- All the zeroes of $f$ are known:

A change of variables can transfer a regular singularity to a removable singularity only if $\nu \in \mathbb{Q} \backslash \mathbb{Z}(\nu \notin \mathbb{Z}$ because we are not in the logarithmic case $)$. Since $\longrightarrow_{E G}$ only changes the exponent differences by an element of $\frac{1}{n} \mathbb{Z}$ (invariance modulo $\frac{1}{n} \mathbb{Z}$, here $n=1), \longrightarrow_{E G}$ can also transfer a regular singularity to a removable singularity only if $\nu \in \mathbb{Q} \backslash \mathbb{Z}$. So in the irrational case $(\nu \in k \backslash \mathbb{Q})$, $\mathrm{S}_{\text {reg }}(\mathrm{L})$ contains all the zeroes of $f$.

2- All the multiplicities of the zeroes of $f$ are known:

For all $s \in \mathrm{S}_{\text {reg }}(\mathrm{L})$, we find the extension $\operatorname{ext}(s)$ of $\mathbb{Q}$ using the exponent differences of $\mathrm{L}$ at $s$. Then we define

$$
\operatorname{ext} 1=\left(\bigcap_{s \in \mathrm{S}_{\mathrm{reg}}(\mathrm{L})} \operatorname{ext}(s)\right) \backslash \mathbb{Q}
$$

Since we have $\nu \notin \mathbb{Q}$ and $\nu$ appears in the exponent differences of $\mathrm{L}$ at any $s \in$ $\mathrm{S}_{\text {reg }}(\mathrm{L})$, we have ext1 $\neq \emptyset$. We choose one element $\sigma$ of ext1. For all $s \in \mathrm{S}_{\text {reg }}(\mathrm{L})$, let $a_{s}$ be the leading coefficient of the exponent differences of $\mathrm{L}$ at $s$ with respect to the variable $\sigma$. Let $m_{s}$ be the multiplicity order of $s$ as zero of $f$. Since we work with $\Delta(\mathrm{L}, s)= \pm 2 m_{s} \cdot \nu+z_{s}$ with $z_{s} \in \mathbb{Z}$ it follows that

$$
a_{s}=2 \beta \cdot m_{s} \text { i.e. } m_{s}=\frac{a_{s}}{2 \beta} \quad \forall s \in \mathrm{S}_{\mathrm{reg}}(\mathrm{L})
$$

where $\beta \in \operatorname{ext} 1 \cup \mathbb{Q}$ is independent of $s$. 
$\mathrm{S}_{\text {reg }}(\mathrm{L})$ contains all the zeroes of $f$, therefore

$$
\begin{aligned}
\operatorname{deg}(\mathrm{A}) & =\sum_{P_{s} \in \overline{\mathrm{S}_{\mathrm{reg}}(\mathrm{L})}} m_{s} \cdot \operatorname{deg}\left(P_{s}\right) \\
\Rightarrow \operatorname{deg}(\mathrm{A}) & =\sum_{P_{s} \in \overline{\mathrm{S}_{\text {reg }}(\mathrm{L})}}\left(\frac{a_{s}}{2 \beta}\right) \cdot \operatorname{deg}\left(P_{s}\right) \text { using }(4.43) \\
\Rightarrow 2 \beta & =\frac{\sum_{P_{s} \in \overline{\mathrm{S}_{\mathrm{reg}}(\mathrm{L})}} a_{s} \cdot \operatorname{deg}\left(P_{s}\right)}{\operatorname{deg}(\mathrm{A})}
\end{aligned}
$$

(4.43) and (4.44) give us

$$
m_{s}=\frac{a_{s}}{\sum_{P_{s} \in \overline{\mathrm{S}_{\text {reg }}(\mathrm{L})}} a_{s} \cdot \operatorname{deg}\left(P_{s}\right)} \cdot \operatorname{deg}(\mathrm{A}) \quad \forall s \in \mathrm{S}_{\mathrm{reg}}(\mathrm{L}) .
$$

So we know all the multiplicities of the zeroes of $f$.

To find candidates for $(\nu, f)$ we proceed as follows:

1. We find all the zeroes of $f$ by computing the set $\mathrm{S}_{\text {reg }}(\mathrm{L})$.

2. For every zero of $f$, we find its multiplicity order by using (4.45) in the proof of Lemma 4.28. So we have A up to a multiplicative constant.

3. Now there is only one unknown coefficients of A that we have to find: the leading coefficient. By Remarks 4.25 we have enough equations to find it.

4. Once we get candidates for $f=\mathrm{A} / \mathrm{B}$, we use Definition 4.10 and Lemma 4.11 to get a list of candidates for $\nu$ related to any $f$.

We have implemented in Maple an algorithm called findBesSqRootIrr to find both candidates for $(\nu, f)$, if they exist. An example for this case can be found in our Maple worksheet associated to this thesis.

\section{Rational case}

Here we are not sure that we know all the zeroes of $f$. The denominator of $\nu$ (denoted by $d$ ) will be very important in that case because $d$ along with the multiplicities will determine whether the singularities disappear. Let $s$ be a root of A with multiplicity $m_{s}$, then $\Delta(\mathrm{L}, s) \equiv \pm m_{s} \nu \bmod \mathbb{Z}$. If $d \mid m_{s}$, the change of variables $x \mapsto f$ can send $s$ to a removable singularity, and then not all the roots of A are known (not all the roots of A are in $\mathrm{S}_{\text {reg }}(\mathrm{L})$ ). We can conclude that if a zero of A becomes a removable singularity, then $m_{s}$ must be a multiple of $d$. 
Definition 4.29. Let us define for $\mathrm{S}_{\text {reg }}(\mathrm{L})$ the two following sets:

$$
\left\{\begin{array}{l}
\mathrm{NS}_{r e g}(\mathrm{~L})=\left\{s \in \mathrm{S}_{\text {reg }}(\mathrm{L}) \mid \exists i \in\{1,2,3\}, \quad \Delta_{i}(\mathrm{~L}, s) \in \mathbb{Q} \backslash \mathbb{Z}\right\}, \\
\operatorname{RemS}_{\text {reg }}(\mathrm{L})=\left\{s \in \mathrm{S}_{\text {reg }}(\mathrm{L}) \mid \Delta_{i}(\mathrm{~L}, s) \in \mathbb{Z} \forall i \in\{1,2,3\}\right\}, \\
\mathcal{A}=\text { set of all the zeroes of } \mathrm{A}, \\
\overline{\mathrm{NS}_{\text {reg }}(\mathrm{L})}=\left\{P_{s} \in k[x] \mid P_{s} \text { is the minimal polynomial of } s \in \mathrm{NS}_{\text {reg }}(\mathrm{L}) \text { over } k\right\}, \\
\overline{\operatorname{RemS}_{\text {reg }}(\mathrm{L})}=\left\{P_{s} \in k[x] \mid P_{s} \text { is the minimal polynomial of } s \in \operatorname{RemS}_{\text {reg }}(\mathrm{L}) \text { over } k\right\}, \\
\overline{\mathcal{A}}=\left\{P_{s} \in k[x] \mid P_{s} \text { is the minimal polynomial of } s \in \mathcal{A} \text { over } k\right\} .
\end{array}\right.
$$

NB: Every time when we will take an element $s$ of $\mathrm{NS}_{\mathrm{reg}}(\mathrm{L}), \Delta(\mathrm{L}, s)$ will represent one of $\Delta_{i}(\mathrm{~L}, s), i \in\{1,2,3\}$, which belongs to $\mathbb{Q} \backslash \mathbb{Z}$.

Remark 4.30. $\operatorname{RemS}_{r e g}(\mathrm{~L})$ and $\mathrm{NS}_{\text {reg }}(\mathrm{L})$ are, respectively, the set of removable and nonremovable singularities of $\mathrm{L}$ in $\mathrm{S}_{\text {reg }}(\mathrm{L})$, and we have $\mathrm{S}_{\text {reg }}(\mathrm{L})=\mathrm{NS}_{\text {reg }}(\mathrm{L}) \cup \operatorname{RemS} \mathrm{S}_{\text {reg }}(\mathrm{L})$.

Lemma 4.31. A can be written in the form $\mathrm{A}=c \mathrm{~A}_{1} \mathrm{~A}_{2}^{d}$ where

$$
\left\{\begin{aligned}
c \in k & \text { and } d=\operatorname{denom}(\nu), \\
\mathrm{A}_{1}= & \prod_{P_{s} \in \overline{\mathcal{A}}} P_{s}^{\beta_{s}} \\
\mathrm{~A}_{2}= & a \prod_{P_{s} \in \overline{\mathcal{A}}} P_{s}^{\alpha_{s}}, \quad \text { with } a \in k, \\
m_{s}= & \text { the multiplicity order of } s \in \mathcal{A} \text { as zero of } f \\
m_{s}= & \alpha_{s} \cdot d+\beta_{s} \text { with } s \in \mathcal{A},\left(\alpha_{s}, \beta_{s}\right) \in \mathbb{N}^{2}, 0 \leq \beta_{s}<d
\end{aligned}\right.
$$

Proof. For $s \in \mathcal{A}$, let $m_{s}$ be the multiplicity order of $s$ as a zero of $f$. We can put $m_{s}$ in the form: $m_{s}=\alpha_{s} \cdot d+\beta_{s}$ with $\left(\alpha_{s}, \beta_{s}\right) \in \mathbb{N}^{2}$ and $0 \leq \beta_{s}<d$.

$$
\begin{aligned}
\mathrm{A} & =b \prod_{P_{s} \in \overline{\mathcal{A}}} P_{s}^{m_{s}}=b \prod_{P_{s} \in \overline{\mathcal{A}}} P_{s}^{\beta_{s}} \cdot \prod_{P_{s} \in \overline{\mathcal{A}}} P_{s}^{\alpha_{s} \cdot d} \text { with } b \in k \\
& =c \prod_{P_{s} \in \overline{\mathcal{A}}} P_{s}^{\beta_{s}} \cdot\left[a \prod_{P_{s} \in \overline{\mathcal{A}}} P_{s}^{\alpha_{s}}\right]^{d}, \quad \text { with } a, c \in k \text { and } b=c \cdot a^{d} .
\end{aligned}
$$

Lemma 4.32. We will take $d=\operatorname{denom}(\nu)>2$.

Proof.

- For $d=1, \nu \in \mathbb{Z}$, and we are in the logarithmic case which has already been treated.

- For $d=2, \nu \in \frac{1}{2}+\mathbb{Z}$, and then $\mathrm{L}_{B^{2}}$ will be reducible. We can solve it by factorization.

Lemma 4.33. Let $d=\operatorname{denom}(\nu)$. 
1- If $\mathrm{NS}_{\text {reg }}(\mathrm{L})=\emptyset$ then $\mathrm{A}_{1}=1, d \mid \operatorname{deg}(\mathrm{A})$ and $\operatorname{deg}\left(\mathrm{A}_{2}\right)=\frac{\operatorname{deg}(\mathrm{A})}{d}$.

2- If $\mathrm{NS}_{r e g}(\mathrm{~L}) \neq \emptyset$ then $\mathrm{A}_{1}=\prod_{P_{s} \in \mathrm{NS}_{\text {reg }}(\mathrm{L})} P_{s}^{\beta_{s}}$ where $m_{s}=\alpha_{s} \cdot d+\beta_{s}$ with $\left(\alpha_{s}, \beta_{s}\right) \in \mathbb{N}^{2}$, $1 \leq \beta_{s}<d$ for $s \in \mathrm{NS}_{\text {reg }}(\mathrm{L})$ and $\frac{m_{s}}{2}$ its multiplicity order as zero of $f$.

Proof. Let $\nu=\frac{a}{d}, \quad a \in \mathbb{Z}, \operatorname{gcd}(a, d)=1$. For any zero $s$ of A, let $m_{s}$ be its multiplicity order as a zero of $f$ and

$$
m_{s}=\alpha_{s} \cdot d+\beta_{s} \text { with }\left(\alpha_{s}, \beta_{s}\right) \in \mathbb{N}^{2}, 0 \leq \beta_{s}<d .
$$

1- Let $s$ be a zero of A. Then $s \in \mathrm{S}_{\text {reg }}(\mathrm{L})=\mathrm{NS}_{\text {reg }}(\mathrm{L}) \cup \operatorname{RemS}_{\text {reg }}(\mathrm{L})$ or $s$ is an apparent singularity or a regular point of L. Hence

$$
\begin{aligned}
\mathrm{NS}_{\mathrm{reg}}(\mathrm{L})=\emptyset & \Rightarrow \Delta(\mathrm{L}, s) \in \mathbb{Z} \Rightarrow m_{s} \nu+z_{s} \in \mathbb{Z} \text { with } z_{s} \in \mathbb{Z} \\
& \Rightarrow\left(\alpha_{s} \cdot d+\beta_{s}\right)\left(\frac{a}{d}\right)+z_{s} \in \mathbb{Z} \Rightarrow \beta_{s} \cdot \frac{a}{d} \in \mathbb{Z}
\end{aligned}
$$

We have $\beta_{s} \cdot \frac{a}{d} \in \mathbb{Z}, \operatorname{gcd}(a, d)=1$ and $0 \leq \beta_{s}<d$. Then, $\beta_{s}=0$. Hence

$$
\mathrm{A}_{1}=\prod_{P_{s} \in \overline{\mathcal{A}}} P_{s}^{\beta_{s}}=\prod_{P_{s} \in \overline{\mathcal{A}}} P_{s}^{0}=1
$$

that means $\operatorname{deg}\left(\mathrm{A}_{1}\right)=0$ and therefore, we have the following implications:

$$
\begin{aligned}
\mathrm{A}=c \mathrm{~A}_{1} \mathrm{~A}_{2}^{d} & \Rightarrow \operatorname{deg}(\mathrm{A})=\operatorname{deg}\left(\mathrm{A}_{1}\right)+\operatorname{deg}\left(\mathrm{A}_{2}\right) \cdot d \Rightarrow \operatorname{deg}(\mathrm{A})=\operatorname{deg}\left(\mathrm{A}_{2}\right) \cdot d \\
& \Rightarrow d \mid d_{\mathrm{A}} \text { and } \operatorname{deg}\left(\mathrm{A}_{2}\right)=\frac{\operatorname{deg}(\mathrm{A})}{d}
\end{aligned}
$$

2- $\quad$ a- Let $s$ be a zero of $\mathrm{A}_{1}$. Then $\beta_{s} \geq 1$ and that means $d \nmid m_{s}$ by (4.46).

$$
\Delta(\mathrm{L}, s)=m_{s} \nu+z_{s}=m_{s}\left(\frac{a}{d}\right)+z_{s} \text { with } z_{s} \in \mathbb{Z} .
$$

$d \nmid m_{s}$ and $\operatorname{gcd}(a, d)=1$ gives us $m_{s}\left(\frac{a}{d}\right) \notin \mathbb{Z}$. So, by $(4.47), \Delta(\mathrm{L}, s) \notin \mathbb{Z}$. Therefore $s \in \mathrm{NS}_{\text {reg }}(\mathrm{L})$.

b- Let $s \in \mathrm{NS}_{\text {reg }}(\mathrm{L})$. Then $\Delta(\mathrm{L}, s) \notin \mathbb{Z}$ by the definition of $\mathrm{NS}_{\text {reg }}(\mathrm{L})$.

$$
\begin{aligned}
& \Rightarrow m_{s} \nu+z_{s} \notin \mathbb{Z} \Rightarrow m_{s}\left(\frac{a}{d}\right)+z_{s} \notin \mathbb{Z} \text { with } z_{s} \in \mathbb{Z} \text {. } \\
& \Rightarrow d \nmid m_{s} \text { since } \operatorname{gcd}(a, d)=1 \\
& \Rightarrow \beta_{s} \neq 0 \text { using }(4.46) \\
& \Rightarrow s \text { is a zero of } \mathrm{A}_{1} .
\end{aligned}
$$

So $\mathrm{A}_{1}=\prod_{P_{s} \in \mathrm{NS}_{\mathrm{reg}}(\mathrm{L})} P_{s}^{\beta_{s}}$.

By the previous lemma, we can conclude that 
Corollary 4.34. A can be written in the form $\mathrm{A}=c \mathrm{~A}_{1} \mathrm{~A}_{2}^{d}$ where

$$
\left\{\begin{array}{l}
c \in k \quad \text { and } d=\text { denom }(\nu), \\
\mathrm{A}_{1}=\left\{\begin{array}{c}
1 \quad \text { if } \mathrm{NS}_{\text {reg }}(\mathrm{L})=\emptyset, \\
\prod_{P_{s} \in \overline{\mathrm{NS}}_{\text {reg }}(\mathrm{L})} P_{s}^{\beta_{s}} \quad \text { otherwise, }
\end{array}\right. \\
\mathrm{A}_{2}=a \prod_{P_{s} \in \overline{\mathcal{A}}} P_{s}^{\alpha_{s}}, \quad \text { with } a \in k, \\
m_{s}=\text { the multiplicity order of } s \in \mathcal{A} \text { as zero of } f, \\
m_{s}=\left\{\begin{array}{cc}
\alpha_{s} \cdot d+\beta_{s} \text { with }\left(\alpha_{s}, \beta_{s}\right) \in \mathbb{N}^{2}, 1 \leq \beta_{s}<d \text { if } s \in \mathrm{NS}_{\text {reg }}(\mathrm{L}), \\
\quad \alpha_{s} \cdot d
\end{array}\right.
\end{array}\right.
$$

Proof. Just use Lemma 4.31 and Lemma 4.33.

Lemma 4.35. Let $s \in \mathcal{A}$ and $d_{s}=\operatorname{denom}(\Delta(\mathrm{L}, s))$. Then we have $d_{s} \mid d$ and therefore,

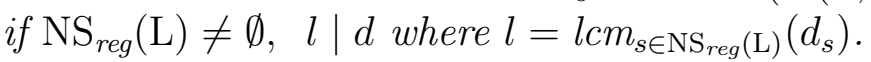

Proof. Let $\nu=\frac{a}{d}, \quad a \in \mathbb{Z}, \operatorname{gcd}(a, d)=1$. Let $s \in \mathcal{A}$ and $m_{s}$ be its multiplicity as a zero of $f$. Then

$$
\begin{aligned}
\Delta(\mathrm{L}, s) & =m_{s} \nu+z_{s}=m_{s}\left(\frac{a}{d}\right)+z_{s} \text { with } z_{s} \in \mathbb{Z} \\
& =\frac{\sigma}{d} \text { where } \sigma=m_{s} \cdot a+d \cdot z_{s} \in \mathbb{Z} .
\end{aligned}
$$

Therefore, $\operatorname{denom}(\Delta(\mathrm{L}, s)) \mid d$ i.e $d_{s} \mid d$.

Let us assume $\mathrm{NS}_{\text {reg }}(\mathrm{L}) \neq \emptyset$. Since $\mathrm{NS}_{\text {reg }}(\mathrm{L}) \subseteq \mathcal{A}$ then $\operatorname{denom}(\Delta(\mathrm{L}, s)) \mid d$ for all $s \in$ $\mathrm{NS}_{\text {reg }}(\mathrm{L})$. That means $\operatorname{lcm}_{s \in \mathrm{NS}_{\mathrm{reg}}(\mathrm{L})}\left(d_{s}\right) \mid d$ i.e $l \mid d$.

Lemma 4.36. Let us assume $\mathrm{NS}_{r e g}(\mathrm{~L}) \neq \emptyset$ and $l=l c m_{s \in \mathrm{NS}_{r e g}(\mathrm{~L})}\left(d_{s}\right)$. Then $\forall s \in \mathrm{NS}_{\text {reg }}(\mathrm{L})$ with $\beta_{s}$ as in Corollary 4.34, $\frac{d}{d_{s}} \mid \beta_{s}$, and therefore $\operatorname{deg}\left(\mathrm{A}_{1}\right) \geq \frac{d}{d_{s}} \cdot \operatorname{deg}\left(P_{s}\right)$ for all $P_{s} \in$ $\overline{\mathrm{NS}_{r e g}(\mathrm{~L})}$ and $\operatorname{deg}\left(\mathrm{A}_{1}\right) \geq \frac{d}{l}$.

Proof. Let $\nu=\frac{a}{d}, a \in \mathbb{Z}, \operatorname{gcd}(a, d)=1$. Let $s \in \mathrm{NS}_{\text {reg }}(\mathrm{L})$ and $m_{s}$ its multiplicity as a zero of $f$ such that $m_{s}=\alpha_{s} \cdot d+\beta_{s}$ with $\left(\alpha_{s}, \beta_{s}\right) \in \mathbb{N}^{2}, 0 \leq \beta_{s}<d$. Then we have $\Delta(\mathrm{L}, s)=m_{s} \nu+z_{s} \notin \mathbb{Z}, \quad z_{s} \in \mathbb{Z}$

$$
\begin{aligned}
& \Rightarrow\left(\alpha_{s} \cdot d+\beta_{s}\right)\left(\frac{a}{d}\right)+z_{s} \notin \mathbb{Z} \\
& \Rightarrow \beta_{s} \frac{a}{d} \notin \mathbb{Z} \text { and } d_{s}=\operatorname{denom}(\Delta(\mathrm{L}, s))=\operatorname{denom}\left(\beta_{s} \frac{a}{d}\right) \quad \text { since } \alpha_{s} a+z_{s} \in \mathbb{Z} .
\end{aligned}
$$

By Lemma 4.35, we have $d_{s} \mid d$. Therefore $d=\varepsilon_{s} \cdot d_{s}$ with $\varepsilon_{s} \in \mathbb{N}$ i.e $\varepsilon_{s}=\frac{d}{d_{s}}$.

$$
\left\{\begin{array} { l } 
{ \beta _ { s } \frac { a } { d } \notin \mathbb { Z } , \quad d = \varepsilon _ { s } \cdot d _ { s } , } \\
{ \operatorname { g c d } ( a , d ) = 1 , \quad d _ { s } = \operatorname { d e n o m } ( \beta _ { s } \frac { a } { d } ) }
\end{array} \Rightarrow \left\{\begin{array}{c}
\varepsilon_{s} \nmid a, \quad d_{s} \nmid a, \\
\varepsilon_{s} \mid \beta_{s} .
\end{array}\right.\right.
$$


Hence $\frac{d}{d_{s}}=\varepsilon_{s} \mid \beta_{s}$. So $\forall s \in \mathrm{NS}_{\mathrm{reg}}(\mathrm{L}), \quad \frac{d}{d_{s}} \mid \beta_{s}$. Let $l=\operatorname{lcm}_{s \in \mathrm{NS}_{\mathrm{reg}}(\mathrm{L})}\left(d_{s}\right)$ and $\forall s \in$ $\mathrm{NS}_{\text {reg }}(\mathrm{L}), \beta_{s}=\sigma_{s} \cdot \frac{d}{d_{s}}$ with $\sigma_{s} \in \mathbb{N}$. Using Corollary $4.34, \beta_{s} \geq 1$ and therefore $\sigma_{s} \geq 1$. Again by Corollary 4.34, we have $\mathrm{A}_{1}=\prod_{P_{s} \in \overline{\mathrm{NS} S_{\mathrm{reg}}(\mathrm{L})}} P_{s}^{\beta_{s}}$

$$
\begin{aligned}
& \Rightarrow \operatorname{deg}\left(\mathrm{A}_{1}\right)=\sum_{P_{s} \in \overline{\mathrm{NS}} \operatorname{reg}(\mathrm{L})} \operatorname{deg}\left(P_{s}\right) \cdot \beta_{s}=\sum_{P_{s} \in \overline{\mathrm{NS}} \operatorname{reg}(\mathrm{L})} \operatorname{deg}\left(P_{s}\right) \cdot \sigma_{s} \cdot \frac{d}{d_{s}} \\
& \Rightarrow \operatorname{deg}\left(\mathrm{A}_{1}\right) \geq \operatorname{deg}\left(P_{s}\right) \cdot \sigma_{s} \cdot \frac{d}{d_{s}} \forall s \in \mathrm{NS}_{\mathrm{reg}}(\mathrm{L}) \quad \operatorname{since} \operatorname{deg}\left(P_{s}\right) \cdot \sigma_{s} \cdot \frac{d}{d_{s}} \geq 0 \quad \forall s \in \mathrm{NS}_{\mathrm{reg}}(\mathrm{L}) \\
& \Rightarrow \operatorname{deg}\left(\mathrm{A}_{1}\right) \geq \operatorname{deg}\left(P_{s}\right) \cdot \frac{d}{d_{s}} \forall s \in \mathrm{NS}_{\mathrm{reg}}(\mathrm{L}) \text { since } \sigma_{s} \geq 1 \\
& \Rightarrow \operatorname{deg}\left(\mathrm{A}_{1}\right) \geq \frac{d}{d_{s}} \forall s \in \mathrm{NS}_{\mathrm{reg}}(\mathrm{L}) \text { since } \operatorname{deg}\left(P_{s}\right) \geq 1 \\
& \Rightarrow \operatorname{deg}\left(\mathrm{A}_{1}\right) \geq \frac{d}{l} \text { since } l \geq d_{s} \quad \forall s \in \mathrm{NS}_{\mathrm{reg}}(\mathrm{L}) .
\end{aligned}
$$

Lemma 4.37. Assume $\mathrm{NS}_{\text {reg }}(\mathrm{L}) \neq \emptyset$ and $l=l c m_{s \in \mathrm{NS}_{\text {reg }}(\mathrm{L})}\left(d_{s}\right)$. If $l \geq \operatorname{deg}(\mathrm{A})$ then we have $\operatorname{deg}(\mathrm{A})=\operatorname{deg}\left(\mathrm{A}_{1}\right)$ and $\operatorname{deg}\left(\mathrm{A}_{2}\right)=0$. That means $\mathrm{A}_{2}=\alpha$ with $\alpha \in k$.

Proof. Let $\mathrm{NS}_{\mathrm{reg}}(\mathrm{L}) \neq \emptyset$ and $l=\operatorname{lcm}_{s \in \mathrm{NS} \text { reg }(\mathrm{L})}\left(d_{s}\right)$. Then $\operatorname{deg}\left(\mathrm{A}_{1}\right) \geq 1$ and by Lemma 4.35, $l \mid d$ where $d=\operatorname{denom}(\nu)$. Hence $d=\sigma \cdot l$ with $\sigma \in \mathbb{N} \backslash\{0\}$ since $d \neq 0$.

$$
\operatorname{deg}(\mathrm{A})=\operatorname{deg}\left(\mathrm{A}_{1}\right)+\operatorname{deg}\left(\mathrm{A}_{2}\right) \cdot d=\operatorname{deg}\left(\mathrm{A}_{1}\right)+\operatorname{deg}\left(\mathrm{A}_{2}\right) \cdot \sigma \cdot l .
$$

Using (4.48) and the fact that $l \geq \operatorname{deg}(\mathrm{A})$ and $\operatorname{deg}\left(\mathrm{A}_{1}\right) \geq 1$ we will get $\operatorname{deg}\left(\mathrm{A}_{2}\right)=0$. Therefore $\operatorname{deg}(\mathrm{A})=\operatorname{deg}\left(\mathrm{A}_{1}\right)$ and $\mathrm{A}_{2}=\alpha$ with $\alpha \in k$.

\section{How to find $d=\operatorname{denom}(\nu)$}

We can find a list of candidates for $d=\operatorname{denom}(\nu)$.

Lemma 4.38. Let us assume $\mathrm{NS}_{\text {reg }}(\mathrm{L})=\emptyset$. Then the candidates for $d=\operatorname{denom}(\nu)$ are

$$
\left\{i \in \mathbb{N} \mid 3 \leq i \leq \frac{d_{\mathrm{A}}}{2} \text { and } i \mid d_{\mathrm{A}}\right\} \bigcup\left\{d_{\mathrm{A}}\right\} \text {. }
$$

Proof. Since $\mathrm{NS}_{\mathrm{reg}}(\mathrm{L})=\emptyset$ then by Lemma 4.33, $d \mid \operatorname{deg}(\mathrm{A})$ and $\operatorname{deg}(\mathrm{A})=\operatorname{deg}\left(\mathrm{A}_{2}\right) \times d$.

1- If $\operatorname{deg}\left(\mathrm{A}_{2}\right)=0$, then $\infty$ is a zero of $f$, otherwise $f$ will be a constant function and that is not good $\left(f^{2} \in k(x) \backslash k\right)$. Let $m_{\infty}$ be the multiplicity order of $\infty$ as zero of $f$. We have $m_{\infty}=\operatorname{deg}(\mathrm{B})$ and $\Delta(\mathrm{L}, \infty) \in \mathbb{Z}$ since $\mathrm{NS}_{\text {reg }}(\mathrm{L})=\emptyset$. So

$$
\begin{aligned}
& d=\operatorname{denom}(\nu)\left|m_{\infty}=\operatorname{deg}(\mathrm{B}) \Rightarrow d\right| d_{\mathrm{A}}=\operatorname{deg}(\mathrm{B}) \text { by Lemma } 4.20 \\
\Rightarrow & d_{\mathrm{A}}=d \cdot \sigma, \quad \sigma \in \mathbb{N} \backslash\{0\} \quad \text { since } d_{\mathrm{A}}=\operatorname{deg}(\mathrm{B}) \neq 0 \Rightarrow d=d_{\mathrm{A}} \text { or } d \leq \frac{d_{\mathrm{A}}}{2} .
\end{aligned}
$$

By Lemma 4.32 we also know that $3 \leq d$. Hence $d$ satisfies

$$
\left(3 \leq d \leq \frac{d_{\mathrm{A}}}{2} \text { and } d \mid d_{\mathrm{A}}\right) \quad \text { or } d=d_{\mathrm{A}} \text {. }
$$


2- If $\operatorname{deg}\left(\mathrm{A}_{2}\right)=1$, then $\operatorname{deg}(\mathrm{A})=\operatorname{deg}\left(\mathrm{A}_{2}\right) \cdot d=d$. Since we don't know $\operatorname{deg}(\mathrm{A})$ but just its bound $d_{\mathrm{A}}, d$ can be taken as $d_{\mathrm{A}}$ : $d=d_{\mathrm{A}}$.

3- If $\operatorname{deg}\left(\mathrm{A}_{2}\right) \geq 2$, then we have $\operatorname{deg}(\mathrm{A})=\operatorname{deg}\left(\mathrm{A}_{2}\right) \cdot d \Rightarrow d=\frac{\operatorname{deg}(\mathrm{A})}{\operatorname{deg}\left(\mathrm{A}_{2}\right)} \leq \frac{\operatorname{deg}(\mathrm{A})}{2}$.

By Lemma 4.32 we also know that $3 \leq d$. So $d$ satisfies

$$
3 \leq d \leq \frac{\operatorname{deg}(\mathrm{A})}{2} \text { and } d \mid \operatorname{deg}(\mathrm{A})
$$

Since we don't know $\operatorname{deg}(\mathrm{A})$ but just its bound $d_{\mathrm{A}}, d$ satisfies

$$
3 \leq d \leq \frac{d_{\mathrm{A}}}{2} \text { and } d \mid d_{\mathrm{A}}
$$

Lemma 4.39. Let us assume $\mathrm{NS}_{r e g}(\mathrm{~L}) \neq \emptyset$ and $d_{s}=\operatorname{denom}(\Delta(\mathrm{L}, s)) \forall s \in \mathrm{NS}_{\text {reg }}(\mathrm{L})$. Let $l=l c m_{s \in \mathrm{NS}_{r e g}(\mathrm{~L})}\left(d_{s}\right)$.

1- If $l \geq d_{\mathrm{A}}$ then the candidates for $d=\operatorname{denom}(\nu)$ are

$$
\left\{i \in \mathbb{N} \mid 3 \leq i \leq d_{\mathrm{A}} \cdot l \text { and } l \mid i\right\}
$$

2- If $l<d_{\mathrm{A}}$, let $d_{\mathrm{A}}$ be on the form $d_{\mathrm{A}}=q \cdot l+r$ with $q, r \in \mathbb{N}, 0 \leq r<l$. Then the candidates for $d=\operatorname{denom}(\nu)$ are

$$
\left\{i \cdot l \mid i \in\{1, \ldots, q\}, \quad \sum_{P_{s} \in \overline{\mathrm{NS}_{r e g}(\mathrm{~L})}} \frac{i \cdot l}{d_{s}} \cdot \operatorname{deg}\left(P_{s}\right) \leq d_{\mathrm{A}} \text { and } g c d_{s \in \mathrm{NS}_{r e g}(\mathrm{~L})}\left(\frac{i \cdot l}{d_{s}}\right) \mid d_{\mathrm{A}}\right\} .
$$

Proof. By Lemma 4.35 we know that $d_{s} \mid d \forall s \in \mathrm{NS}_{\text {reg }}(\mathrm{L})$ and $l \mid d$.

1- Let us assume $l \geq d_{\mathrm{A}}$. By Lemma 4.37 and Lemma 4.36 we have, respectively, $d_{\mathrm{A}}=\operatorname{deg}\left(\mathrm{A}_{1}\right)$ and $d / l \leq \operatorname{deg}\left(\mathrm{A}_{1}\right)$. So $d / l \leq d_{\mathrm{A}}$ i.e $d \leq d_{\mathrm{A}} \cdot l$. We also know by Lemma 4.32 that $d \geq 3$. Therefore we have

$$
3 \leq d \leq d_{\mathrm{A}} \cdot l \text { and } l \mid d
$$

So the candidates for $d=\operatorname{denom}(\nu)$ are

$$
\left\{i \in \mathbb{N} \mid 3 \leq i \leq d_{\mathrm{A}} \cdot l \text { and } l \mid i\right\}
$$

2- Let us assume $l<d_{\mathrm{A}} . l \mid d$ and $d \geq 3$ by Lemma 4.32 then $d=l \cdot i, i \in \mathbb{N}$ and $i \geq 1$. So $\operatorname{deg}(\mathrm{A})=\operatorname{deg}\left(\mathrm{A}_{2}\right) \cdot d+\operatorname{deg}\left(\mathrm{A}_{1}\right)=\operatorname{deg}\left(\mathrm{A}_{2}\right) \cdot l \cdot i+\operatorname{deg}\left(\mathrm{A}_{1}\right)$. Since we don't know $\operatorname{deg}(\mathrm{A})$ but just $d_{\mathrm{A}}$, then we can take

$$
d_{\mathrm{A}}=\operatorname{deg}\left(\mathrm{A}_{2}\right) \cdot l \cdot i+\operatorname{deg}\left(\mathrm{A}_{1}\right), \quad i \geq 1 .
$$

On the other hand, let $d_{\mathrm{A}}$ have the form

$$
d_{\mathrm{A}}=q \cdot l+r, \quad \text { with } q, r \in \mathbb{N}, 0 \leq r<l .
$$


(4.49) and (4.50) imply

$$
i \in\{1, \ldots, q\}
$$

Let $d_{1}=\sum_{P_{s} \in \overline{\mathrm{NS}}} \frac{d}{\mathrm{~N}_{\mathrm{reg}}(\mathrm{L})} \cdot \operatorname{deg}\left(P_{s}\right)=\sum_{P_{s} \in \overline{\mathrm{NS}}} \frac{l \cdot i}{d_{\mathrm{reg}}(\mathrm{L})} \cdot \operatorname{deg}\left(P_{s}\right)$. By Lemma 4.36 we get $\frac{d}{d_{s}} \mid \beta_{s} \forall s \in \mathrm{NS}_{\text {reg }}(\mathrm{L})$. Then $\forall s \in \mathrm{NS}_{\text {reg }}(\mathrm{L}) \quad \beta_{s}=\sigma_{s} \cdot \frac{d}{d_{s}}, \quad$ with $\sigma_{s} \in \mathbb{N}$ and $\sigma_{s} \geq 1$ because $\beta_{s} \neq 0$.

$$
\begin{aligned}
d_{\mathrm{A}} & =\operatorname{deg}\left(\mathrm{A}_{2}\right) \cdot l \cdot i+\operatorname{deg}\left(\mathrm{A}_{1}\right)=\operatorname{deg}\left(\mathrm{A}_{2}\right) \cdot l \cdot i+\sum_{P_{s} \in \overline{\mathrm{NS}} \operatorname{seg}(\mathrm{L})} \beta_{s} \cdot \operatorname{deg}\left(P_{s}\right) \\
& =\operatorname{deg}\left(\mathrm{A}_{2}\right) \cdot l \cdot i+\sum_{P_{s} \in \overline{\mathrm{NS}}(\mathrm{L})} \sigma_{s} \cdot \frac{d}{d_{s}} \cdot \operatorname{deg}\left(P_{s}\right) \\
& \geq \sum_{P_{s} \in \overline{\mathrm{NS}} \mathrm{reg}(\mathrm{L})} \sigma_{s} \cdot \frac{d}{d_{s}} \cdot \operatorname{deg}\left(P_{s}\right) \text { since } \operatorname{deg}\left(\mathrm{A}_{2}\right) \cdot l \cdot i \geq 0 \\
& \geq \sum_{P_{s} \in \overline{\mathrm{NS}}} \frac{d}{d_{s e g}(\mathrm{~L})} \cdot \operatorname{deg}\left(P_{s}\right) \text { since } \sigma_{s} \geq 1 \quad \forall s \in \mathrm{NS}_{\mathrm{reg}}(\mathrm{L}) \\
\Rightarrow & d_{\mathrm{A}} \geq d_{1} .
\end{aligned}
$$

Let $d_{2}=\operatorname{gcd}_{s \in \mathrm{NS}_{\mathrm{reg}}(\mathrm{L})}\left(\frac{d}{d_{s}}\right)=\operatorname{gcd}_{s \in \mathrm{NS} \operatorname{reg}(\mathrm{L})}\left(\frac{i \cdot l}{d_{s}}\right) . \forall s \in \mathrm{NS}_{\mathrm{reg}}(\mathrm{L})$, Since $d_{2} \mid \frac{d}{d_{s}}$ and $\frac{d}{d_{s}} \mid \beta_{s}$, we get $d_{2} \mid \beta_{s}$. Therefore $d_{2} \mid \operatorname{deg}\left(\mathrm{A}_{1}\right)$ because

$$
\begin{aligned}
\operatorname{deg}\left(\mathrm{A}_{1}\right)= & \sum_{P_{s} \in \mathrm{NS}_{\mathrm{reg}}(\mathrm{L})} \beta_{s} \cdot \operatorname{deg}\left(P_{s}\right) \\
& d_{2} \mid \frac{d}{d_{s}} \quad \forall s \in \mathrm{NS}_{\mathrm{reg}}(\mathrm{L}) \Rightarrow \frac{d}{d_{s}}=b_{s} \cdot d_{2} \quad \text { with } b_{s} \in \mathbb{N} \quad \forall s \in \mathrm{NS}_{\mathrm{reg}}(\mathrm{L}) \\
\Rightarrow & d=\left(d_{s} \cdot b_{s}\right) \cdot d_{2} \quad \forall s \in \mathrm{NS}_{\mathrm{reg}}(\mathrm{L}) \Rightarrow d_{2} \mid d
\end{aligned}
$$

We have $d_{2}\left|\operatorname{deg}\left(\mathrm{A}_{1}\right), d_{2}\right| d$ and $d_{\mathrm{A}}=\operatorname{deg}\left(\mathrm{A}_{2}\right) \cdot d+\operatorname{deg}\left(\mathrm{A}_{1}\right)$, therefore

$$
d_{2} \mid d_{\mathrm{A}}
$$

Using (4.50), (4.51), (4.52) and (4.53), the candidates for $d$ are $i \cdot l$ such that

$$
\left\{\begin{array}{l}
d_{\mathrm{A}}=q \cdot l+r, \quad \text { with } q, r \in \mathbb{N}, 0 \leq r<l \\
i \in\{1, \ldots, q\}, \\
\quad \sum_{P_{s} \in \overline{\mathrm{NS}} \operatorname{reg}(\mathrm{L})} \frac{i \cdot l}{d_{s}} \cdot \operatorname{deg}\left(P_{s}\right) \leq d_{\mathrm{A}} \text { and } \operatorname{gcd}_{s \in \mathrm{NS}_{\mathrm{reg}}(\mathrm{L})}\left(\frac{i \cdot l}{d_{s}}\right) \mid d_{\mathrm{A}} .
\end{array}\right.
$$




\section{$\underline{\text { How to find the degree of } A_{1} \text { and } A_{2}}$}

Let us first see how to get a list of candidates for $\operatorname{deg}\left(\mathrm{A}_{2}\right)$.

Lemma 4.40. Let $d=\operatorname{denom}(\nu)$.

1- If $\mathrm{NS}_{\text {reg }}(\mathrm{L})=\emptyset$, then we have $\operatorname{deg}\left(\mathrm{A}_{2}\right)=\frac{d_{\mathrm{A}}}{d}$.

2- If $\mathrm{NS}_{r e g}(\mathrm{~L}) \neq \emptyset$, let $d_{\mathrm{A}}=q \cdot d+r$, with $q, r \in \mathbb{N}, 0 \leq r<d$. Then we have $\operatorname{deg}\left(\mathrm{A}_{2}\right) \in\{0, \ldots, q\}$

Proof. Let $d=\operatorname{denom}(\nu)$.

1- Let us assume $\mathrm{NS}_{\text {reg }}(\mathrm{L})=\emptyset$. We have by Lemma $4.33 d_{\mathrm{A}}=\operatorname{deg}\left(\mathrm{A}_{2}\right) \cdot d$. So we have $\operatorname{deg}\left(\mathrm{A}_{2}\right)=\frac{d_{\mathrm{A}}}{d}$

2- Let us assume $\mathrm{NS}_{\text {reg }}(\mathrm{L}) \neq \emptyset$ and $d_{\mathrm{A}}=q \cdot d+r, \quad$ with $q, r \in \mathbb{N}, 0 \leq r<d$. Since we also have $d_{\mathrm{A}}=\operatorname{deg}\left(\mathrm{A}_{2}\right) \cdot d+\operatorname{deg}\left(\mathrm{A}_{1}\right)$ then we can take the candidates for $\operatorname{deg}\left(\mathrm{A}_{2}\right)$ in the set $\{0, \ldots, q\}$.

When we find a candidate for $\operatorname{deg}\left(\mathrm{A}_{2}\right)$ we can also get its associated candidate for $\operatorname{deg}\left(\mathrm{A}_{1}\right)$.

Corollary 4.41. Let us assume that we know $\operatorname{deg}\left(\mathrm{A}_{2}\right)$, then by using the relation

$$
d_{\mathrm{A}}=\operatorname{deg}\left(\mathrm{A}_{2}\right) \cdot d+\operatorname{deg}\left(\mathrm{A}_{1}\right)
$$

we also have $\operatorname{deg}\left(\mathrm{A}_{1}\right)$.

Proof. Since we know $\operatorname{deg}\left(\mathrm{A}_{2}\right)$, we just use the relation $d_{\mathrm{A}}=\operatorname{deg}\left(\mathrm{A}_{2}\right) \cdot d+\operatorname{deg}\left(\mathrm{A}_{1}\right)$ and get

$$
\operatorname{deg}\left(\mathrm{A}_{1}\right)=d_{\mathrm{A}}-\operatorname{deg}\left(\mathrm{A}_{2}\right) \cdot d
$$

\section{How to find $\mathrm{A}_{1}$}

For $\mathrm{NS}_{\text {reg }}(\mathrm{L})=\emptyset$, we can use Lemma 4.33 to get $\mathrm{A}_{1}: \mathrm{A}_{1}=1$. The problem now is what to do if $\mathrm{NS}_{\text {reg }}(\mathrm{L}) \neq \emptyset$. In that case, also by Lemma 4.33, we know that $\mathrm{A}_{1}=\prod_{P_{s} \in \mathrm{NS}_{\mathrm{reg}}(\mathrm{L})} P_{s}^{\beta_{s}}$. Hence, finding $\mathrm{A}_{1}$ is equivalent to find $\beta_{s} \forall s \in \mathrm{NS}_{\mathrm{reg}}(\mathrm{L})$.

We will see in this lemma which technique can be used to find all the $\beta_{s}$ with $s \in$ $\mathrm{NS}_{\text {reg }}(\mathrm{L})$.

Lemma 4.42. Let $d=\operatorname{denom}(\nu)$. Let us assume $\mathrm{NS}_{\text {reg }}(\mathrm{L}) \neq \emptyset$ and $d_{s}=\operatorname{denom}(\Delta(\mathrm{L}, s))$ for all $s \in \mathrm{NS}_{\text {reg }}(\mathrm{L})$. Then we can find $\beta_{s} \forall s \in \mathrm{NS}_{\text {reg }}(\mathrm{L})$ by solving the Diophantine equation:

$$
\sum_{P_{s} \in \overline{\mathrm{NS}}(\mathrm{L})} \beta_{s} \cdot \operatorname{deg}\left(P_{s}\right)=\operatorname{deg}\left(\mathrm{A}_{1}\right) \text { with }\left\{\begin{array}{l}
1 \leq \beta_{s}<d \\
\frac{d}{d_{s}} \mid \beta_{s} .
\end{array}\right.
$$


Proof. By Lemma 4.33 we have $\mathrm{A}_{1}=\prod_{P_{s} \in \prod_{\mathrm{NS} \text { reg }(\mathrm{L})}} P_{s}^{\beta_{s}}, 1 \leq \beta_{s}<d$. Then

$$
\operatorname{deg}\left(\mathrm{A}_{1}\right)=\sum_{P_{s} \in \overline{\mathrm{NS} \operatorname{reg}(\mathrm{L})}} \beta_{s} \cdot \operatorname{deg}\left(P_{s}\right)
$$

By Lemma 4.36 we have

$$
\frac{d}{d_{s}} \mid \beta_{s} \text { for all } s \in \mathrm{NS}_{\mathrm{reg}}(\mathrm{L}) .
$$

Since we know $d$, $\operatorname{deg}\left(\mathrm{A}_{1}\right)$ and $d_{s} \forall s \in \mathrm{NS}_{\text {reg }}(\mathrm{L})$, all the $\beta_{s}$ with $s \in \mathrm{NS}_{\text {reg }}(\mathrm{L})$ will be solutions of this Diophantine equation

$$
\sum_{P_{s} \in \overline{\mathrm{NS}} \operatorname{reg}(\mathrm{L})} \beta_{s} \cdot \operatorname{deg}\left(P_{s}\right)=\operatorname{deg}\left(\mathrm{A}_{1}\right) \text { with }\left\{\begin{array}{l}
1 \leq \beta_{s}<d \\
\frac{d}{d_{s}} \mid \beta_{s}
\end{array}\right.
$$

Let us see now how to solve our Diophantine equation and find all $\beta_{s}, s \in \mathrm{NS}_{\mathrm{reg}}(\mathrm{L})$. This will lead to find candidates for $\mathrm{A}_{1}$.

Lemma 4.43. Let us assume $\mathrm{NS}_{r e g}(\mathrm{~L}) \neq \emptyset$. Then we can solve the Diophantine equation of Lemma 4.42 and get a list of candidates for $\mathrm{A}_{1}$.

Proof. Let $d=\operatorname{denom}(\nu)$. For $s \in \mathrm{NS}_{\text {reg }}(\mathrm{L})$, let $d_{s}=\operatorname{denom}(\Delta(\mathrm{L}, s)), m_{s}$ the multiplicity order of $s$ as a zero of $f$ and put $m_{s}$ in the form

$$
m_{s}=\alpha_{s} \cdot d+\beta_{s} \text { with }\left(\alpha_{s}, \beta_{s}\right) \in \mathbb{N}^{2}, 1 \leq \beta_{s}<d .
$$

By Lemma 4.36, $\forall s \in \mathrm{NS}_{\text {reg }}(\mathrm{L})$

$$
\begin{aligned}
& \frac{d}{d_{s}} \mid \beta_{s} \Rightarrow \beta_{s}=\frac{d}{d_{s}} \cdot \sigma_{s} \text { with } \sigma_{s} \in \mathbb{N} \Rightarrow 1 \leq \frac{d}{d_{s}} \cdot \sigma_{s}<d \quad \text { by }(4.55) \\
& \Rightarrow \sigma_{s} \geq 1 \text {. } \\
& \mathrm{A}_{1}=\prod_{P_{s} \in \overline{\mathrm{N} \mathrm{S}_{\mathrm{reg}}(\mathrm{L})}} P_{s}^{\beta_{s}}=\prod_{P_{s} \in \overline{\mathrm{N}} \mathrm{reg}(\mathrm{L})} P_{s}^{\left(d / d_{s}\right) \cdot \sigma_{s}} \\
& \Rightarrow \operatorname{deg}\left(\mathrm{A}_{1}\right)=\sum_{P_{s} \in \overline{\mathrm{NS}} \mathrm{reg}_{\mathrm{reg}}(\mathrm{L})} \frac{d}{d_{s}} \cdot \sigma_{s} \cdot \operatorname{deg}\left(P_{s}\right) \geq \sum_{P_{s} \in \overline{\mathrm{NS}}} \frac{d}{d_{\mathrm{reg}}(\mathrm{L})} \cdot \operatorname{deg}\left(P_{s}\right) \quad\left(\sigma_{s} \geq 1 \forall s \in \mathrm{NS}_{\mathrm{reg}}(\mathrm{L})\right) \\
& \Rightarrow \operatorname{deg}\left(\mathrm{A}_{1}\right) \geq \frac{d}{d_{s}} \cdot \operatorname{deg}\left(P_{s}\right) \quad \forall P_{s} \in \overline{\mathrm{NS}_{\mathrm{reg}}(\mathrm{L})} \text { since } \frac{d}{d_{s}} \cdot \operatorname{deg}\left(P_{s}\right) \geq 0 \quad \forall P_{s} \in \overline{\mathrm{NS}_{\mathrm{reg}}(\mathrm{L})} \\
& \Rightarrow \operatorname{deg}\left(\mathrm{A}_{1}\right)-\frac{d}{d_{s}} \cdot \operatorname{deg}\left(P_{s}\right) \geq 0 \quad \forall P_{s} \in \overline{\mathrm{NS}_{\mathrm{reg}}(\mathrm{L})} \text {. }
\end{aligned}
$$

Let us take one element $s_{1} \in \mathrm{NS}_{\text {reg }}(\mathrm{L})$. By (4.56) we have

$$
\operatorname{deg}\left(\mathrm{A}_{1}\right)-\frac{d}{d_{s_{1}}} \cdot \operatorname{deg}\left(P_{s_{1}}\right) \geq 0
$$




$$
\begin{aligned}
& \operatorname{deg}\left(\mathrm{A}_{1}\right)=\sum_{P_{s} \in \overline{\mathrm{NS}} \operatorname{reg}(\mathrm{L})} \beta_{s} \cdot \operatorname{deg}\left(P_{s}\right) \\
& \Rightarrow \operatorname{deg}\left(\mathrm{A}_{1}\right)-\sum_{P_{s} \in \overline{\mathrm{NS}} \mathrm{reg}_{\mathrm{reg}}(\mathrm{L})\left\{P_{s_{1}}\right\}} \beta_{s} \cdot \operatorname{deg}\left(P_{s}\right)=\beta_{s_{1}} \cdot \operatorname{deg}\left(P_{s_{1}}\right) \\
& \Rightarrow \operatorname{deg}\left(\mathrm{A}_{1}\right)-\sum_{P_{s} \in \overline{\mathrm{NS}} \mathrm{Seg}_{\mathrm{reg}}(\mathrm{L}) \backslash\left\{P_{s_{1}}\right\}} \operatorname{deg}\left(P_{s}\right) \geq \beta_{s_{1}} \cdot \operatorname{deg}\left(P_{s_{1}}\right) \quad \text { since } \beta_{s} \geq 1 \quad \forall s \in \mathrm{NS}_{\mathrm{reg}}(\mathrm{L})
\end{aligned}
$$

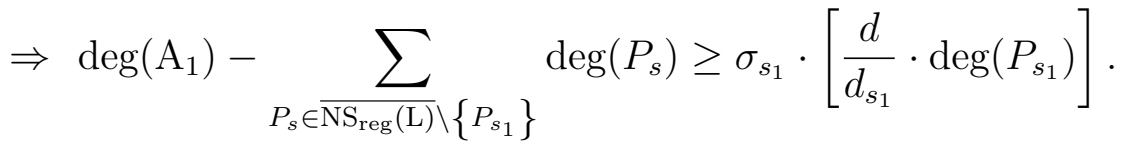

On the other side, let

$$
\begin{gathered}
\operatorname{deg}\left(\mathrm{A}_{1}\right)-\sum_{P_{s} \in \overline{\mathrm{NS}} \mathrm{S}_{\mathrm{reg}}(\mathrm{L}) \backslash\left\{P_{s_{1}}\right\}} \operatorname{deg}\left(P_{s}\right)=q \cdot\left[\frac{d}{d_{s_{1}}} \cdot \operatorname{deg}\left(P_{s_{1}}\right)\right]+r \text { with } q, r \in \mathbb{N} \\
\text { and } 0 \leq r<\frac{d}{d_{s_{1}}} \cdot \operatorname{deg}\left(P_{s_{1}}\right) .
\end{gathered}
$$

Then we can take, because of $(4.58), \sigma_{s_{1}} \in\{0, \ldots, q\}$. The fact that $\sigma_{s_{1}} \geq 1$ reduces this set to

$$
\begin{gathered}
\sigma_{s_{1}} \in\{1, \ldots, q\} \\
\beta_{s_{1}}<d \Rightarrow \frac{d}{d_{s_{1}}} \cdot \sigma_{s_{1}}<d .
\end{gathered}
$$

Since we know $d, \operatorname{deg}\left(\mathrm{A}_{1}\right)$ and $d_{s} \forall s \in \mathrm{NS}_{\text {reg }}(\mathrm{L})$, using (4.57), (4.59), (4.60) and (4.61) we can find candidates for $\sigma_{s_{1}}$ by solving

$$
\left\{\begin{array}{c}
\operatorname{deg}\left(\mathrm{A}_{1}\right)-\sum_{P_{s} \in \overline{\mathrm{NS}} \mathrm{S}_{\mathrm{reg}}(\mathrm{L}) \backslash\left\{P_{s_{1}}\right\}} \operatorname{deg}\left(P_{s}\right)=q \cdot\left[\frac{d}{d_{s_{1}}} \cdot \operatorname{deg}\left(P_{s_{1}}\right)\right]+r \text { with } q, r \in \mathbb{N} \\
\text { and } 0 \leq r<\frac{d}{d_{s_{1}}} \cdot \operatorname{deg}\left(P_{s_{1}}\right), \\
\sigma_{s_{1}} \in\{1, \ldots, q\}, \quad \sigma_{s_{1}} \cdot \frac{d}{d_{s_{1}}}<d, \quad \text { and } \operatorname{deg}\left(\mathrm{A}_{1}\right)-\frac{d}{d_{s_{1}}} \cdot \operatorname{deg}\left(P_{s_{1}}\right) \geq 0 .
\end{array}\right.
$$

Therefore, by using the relation $\beta_{s_{1}}=\frac{d}{d_{s_{1}}} \cdot \sigma_{s_{1}}$, we get also the candidates for $\beta_{s_{1}}$.

We continue the process by considering

$$
\mathrm{A}_{1}=\prod_{P_{s} \in \overline{\mathrm{NS}} \operatorname{Seg}_{\mathrm{reg}}(\mathrm{L}) \backslash\left\{P_{s_{1}}\right\}} P_{s}^{\beta_{s}}, \quad 1 \leq \beta_{s}<d .
$$

That means $\operatorname{deg}\left(\mathrm{A}_{1}\right)=\operatorname{deg}\left(\mathrm{A}_{1}\right)-\frac{d}{d_{s_{1}}} \cdot \sigma_{s_{1}} \cdot \operatorname{deg}\left(P_{s_{1}}\right)$, and we take another $s_{2} \in \mathrm{NS}_{\text {reg }}(\mathrm{L})$. At the end, we will find all the candidates for $\beta_{s}, \forall s \in \mathrm{NS}_{\text {reg }}(\mathrm{L})$.

Since $\mathrm{A}_{1}=\prod_{P_{s} \in \overline{\mathrm{NS} \mathrm{S}_{\mathrm{reg}}(\mathrm{L})}} P_{s}^{\beta_{s}}$, we have also the candidates for $\mathrm{A}_{1}$. 


\section{How to find $c$ such that $\mathrm{A}=c \mathrm{~A}_{1} \mathrm{~A}_{2}^{d}$}

We have seen how to find $\mathrm{A}_{1}, \mathrm{~B} \in k[x]$. That means we have $\mathrm{A}_{1} / \mathrm{B} \in k(x)$ and $f \cdot \mathrm{B} / \mathrm{A}_{1} \in$ $k[x]$. So $c \mathrm{~A}_{2}^{d} \in k[x]$. This doesn't imply that $\mathrm{A}_{2} \in k[x]$. To get $\mathrm{A}_{2} \in k[x]$, as in our assumption, we choose $c \in k$ by the following method.

Case 1: $\quad(k \cup\{\infty\}) \cap \mathrm{S}_{\text {irr }}(\mathrm{L}) \neq \emptyset$.

We have here $\Delta(\mathrm{L}, s) \in k\left(t_{s}\right) \forall s \in(k \cup\{\infty\}) \cap \mathrm{S}_{\text {irr }}(\mathrm{L})$ and we can compute a truncated series for $f=\left(c \mathrm{~A}_{1} \mathrm{~A}_{2}^{d}\right) / \mathrm{B}$ at $x=s$. Therefore, we have a truncated series for $f \cdot \mathrm{B} / \mathrm{A}_{1}$ (which equals $c \mathrm{~A}_{2}^{d}$ ) at $x=s$. If we want to have $\mathrm{A}_{2}^{d}$ monic then we have to take $c$ as the coefficient of the first term of this series (the truncated series of $f \cdot \mathrm{B} / \mathrm{A}_{1}$ at $x=s$ ). That implies the truncated series of $\mathrm{A}_{2}$ at $x=s$ has first term 1 (or another $d$-th root of 1 in $k$ ). Hence we can construct other terms of $\mathrm{A}_{2}$ by Hensel Lifting (see [42]). By the construction method, $\mathrm{A}_{2}$ will be in $k[x]$.

The problem we face now is the following: if we choose other $\widetilde{c}$ such that $\mathrm{A}_{2} \in k[x]$, will that lead to the same candidates for $f$ ?

Definition 4.44. We say that $c_{1}$ and $c_{2}$ are equivalent $\left(c_{1} \sim c_{2}\right)$, if $c_{1}=c_{2} \cdot c^{d}$ where $c \in k$.

Lemma 4.45. Assume $k \cup\{\infty\}) \cap \mathrm{S}_{\text {irr }}(\mathrm{L}) \neq \emptyset . \mathrm{A}_{1}$ and $\mathrm{B}$, which are monic and in $k[x]$, are fixed. Let $s \in k \cup\{\infty\}) \cap \mathrm{S}_{\text {irr }}(\mathrm{L})$ and $c_{1}, c_{2}$ and $\mathrm{A}_{2}$ be computed by the method we introduced in case 1 above and theorem below such that $\left(c_{1} \mathrm{~A}_{1} \mathrm{~A}_{2}^{d}\right) / \mathrm{B}$ and $\left(c_{2} \mathrm{~A}_{1} \widetilde{\mathrm{A}}_{2}^{d}\right) / \mathrm{B}$ are candidates for $f$ with $\mathrm{A}_{2}, \widetilde{\mathrm{A}}_{2} \in k[x]$. Then $c_{1}$ and $c_{2}$ lead to the same candidates if and only if $c_{1} \sim c_{2}$.

Proof.

1- Assume that $c_{1}$ and $c_{2}$ lead to the same candidates for $f$. That means we will have

$$
\frac{c_{1} \mathrm{~A}_{1} \mathrm{~A}_{2}^{d}}{\mathrm{~B}}=\frac{c_{2} \mathrm{~A}_{1} \widetilde{\mathrm{A}}_{2}^{d}}{\mathrm{~B}} \Rightarrow c_{1} \mathrm{~A}_{2}^{d}=c_{2} \widetilde{\mathrm{A}}_{2}^{d} \Rightarrow \frac{c_{1}}{c_{2}}=\frac{\widetilde{\mathrm{A}}_{2}^{d}}{\mathrm{~A}_{2}^{d}} \in k \Rightarrow \mathrm{A}_{2}=c \widetilde{\mathrm{A}}_{2} \text { with } c \in k,
$$

such that $c^{d}=c_{1} / c_{2}$. So $c_{2} \cdot c^{d}=c_{1}$ and then $c_{1} \sim c_{2}$.

2- Assume that $c_{1} \sim c_{2}$. That means $c_{1}=c_{2} \cdot c^{d}$.

$$
\frac{c_{1} \mathrm{~A}_{1} \mathrm{~A}_{2}^{d}}{\mathrm{~B}}=\frac{c_{2} \cdot c^{d} \mathrm{~A}_{1} \mathrm{~A}_{2}^{d}}{\mathrm{~B}}=\frac{c_{2} \mathrm{~A}_{1}\left(c \mathrm{~A}_{2}\right)^{d}}{\mathrm{~B}}=\frac{c_{2} \mathrm{~A}_{1} \widetilde{\mathrm{A}}_{2}^{d}}{\mathrm{~B}} \text { with } \widetilde{\mathrm{A}}_{2}=c \mathrm{~A}_{2} \in k[x] .
$$

So $\left(c_{1} \mathrm{~A}_{1} \mathrm{~A}_{2}^{d}\right) / \mathrm{B}$ gotten from $c_{1}$ can also be gotten from $c_{2}$. Therefore $c_{1}$ and $c_{2}$ lead to the same candidates for $f$.

Conclusion : If we choose other $\widetilde{c}$ such that $\mathrm{A}_{2} \in k[x]$, then it will lead to the same candidates of $f$. So our method is sufficient in this case.

Case 2: $\quad(k \cup\{\infty\}) \cap \mathrm{S}_{\text {irr }}(\mathrm{L})=\emptyset$

For some $s \in \mathrm{S}_{\text {irr }}(\mathrm{L})$, we can temporarily extend the field $k$ to $k(s)$ and recompute the local data over the new field. Then we compute all candidates $g \in k(s, x)$ for $f$ as in case 1 . Any candidate $g$ for $f$ that is defined over $k(x)$ will be discarded without further computation (see the last part "Algebraic Extension"). 
Remark 4.46. In the case 2, sometimes we can still use the way in case 1 to guess the value of $c$. But it might not lead to the correct candidates because c might not be unique (up to multiplication by a d-th power) over $k$. So we need to introduce algebraic extensions (see the last part "Algebraic Extension").

\section{How to find $\mathrm{A}_{2}$}

In order to find $\mathrm{A}$ in $f=\frac{c \mathrm{~A}_{1} \mathrm{~A}_{2}^{d}}{\mathrm{~B}}$, the only unknown part is now $\mathrm{A}_{2}$.

Lemma 4.47. To recover $\mathrm{A}_{2}$ we only need $\frac{1}{3} d_{\mathrm{A}}+1$ linear equations for its coefficients.

Proof. Let $d=\operatorname{denom}(\nu)$. By Lemma $4.32 d \geq 3$.

$$
\begin{aligned}
& d_{\mathrm{A}}=\operatorname{deg}\left(\mathrm{A}_{1}\right)+\operatorname{deg}\left(\mathrm{A}_{2}\right) \cdot d \Rightarrow \operatorname{deg}\left(\mathrm{A}_{2}\right) \cdot d \leq d_{\mathrm{A}} \Rightarrow \operatorname{deg}\left(\mathrm{A}_{2}\right) \leq \frac{d_{\mathrm{A}}}{d} \\
\Rightarrow & \operatorname{deg}\left(\mathrm{A}_{2}\right) \leq \frac{d_{\mathrm{A}}}{3} \text { since } d \geq 3 .
\end{aligned}
$$

Hence to get $\mathrm{A}_{2}$ we only need $\frac{1}{3} d_{\mathrm{A}}+1$ linear equations for its coefficients.

Let $\mathrm{A}_{2}=\sum_{i=0}^{\operatorname{deg}\left(\mathrm{A}_{2}\right)} b_{i} x^{i}, \quad b_{i} \in k$. By using the same methods as in Lemma 4.22 and Lemma 4.24, the equations we get for $\left\{b_{i}\right\}$ will not be linear because we need to evaluate the $d$-th power. We use here the method of Hensel Lifting (see [42]) to find linear equations for the coefficients $\left\{b_{i}\right\}$ of $\mathrm{A}_{2}$.

Theorem 4.48. Let $\mathrm{A}_{2}$ in $\mathrm{A}=c \mathrm{~A}_{1} \mathrm{~A}_{2}^{d}$ be on the form $\mathrm{A}_{2}=\sum_{i=0}^{\operatorname{deg}\left(\mathrm{A}_{2}\right)} b_{i} x^{i}, \quad b_{i} \in k$. For each $s \in \mathrm{S}_{i r r}(\mathrm{~L})$ with $m_{s}$ as its multiplicity order as a pole of $f$, we will get $\left\lceil m_{s}\right\rceil$ linear equations for $\left\{b_{i}\right\}$.

Proof. The proof is similar as the proof of theorem 10 in [42].

Remark 4.49. If $s \notin k$, we can use the results from Lemma 4.22 and Lemma 4.24 to get equations. Therefore, we can always obtain $\geq \frac{1}{2} d_{\mathrm{A}}$ linear equations, while $\left\lfloor\frac{1}{3} d_{\mathrm{A}}\right\rfloor+1$ equations are sufficient. So we always get enough linear equations.

\section{How to find the Bessel parameter $\nu$}

We take $\nu=a / d, a \in \mathbb{Z}, d \in \mathbb{N} \backslash\{0\}$ and $\operatorname{gcd}(a, d)=1$. We know how to find $d=\operatorname{denom}(\nu)$. What remains now is how to find $a$.

Since we just have to take $\nu$ modulo $\mathbb{Z}$, then $a \in[-d, d] \cap \mathbb{Z}$. That is also equivalent to $a \in[-d / 2, d / 2] \cap \mathbb{Z}$. We also know that $a / d \notin \mathbb{Z}$, if not we are in the logarithmic case. Then $a \in(]-d / 2,-1] \cup[1, d / 2[) \cap \mathbb{Z}$. Since taking $\nu$ or $-\nu$ is the same for our operator $\mathrm{L}_{\check{B}_{\nu}^{2}}, a \in[1, d / 2[\cap \mathbb{N}$. Let

$$
V_{1}=\left\{\frac{a}{d} \mid a \in\left[1, \frac{d}{2}[\cap \mathbb{N}\}\right.\right.
$$


If $\mathrm{S}_{\mathrm{reg}}(\mathrm{L}) \neq \emptyset$, using Definition 4.10 and Lemma 4.11, we can find $\mathrm{N}$, the set of candidates for $\nu$ modulo $\mathbb{Z}$. Therefore, the reduced set of candidates for $\nu$ is

$$
\mathrm{V}=V_{1} \cap \mathrm{N}
$$

where " $\cap$ " represents the intersection modulo $\mathbb{Z}$.

We have implemented in Maple an algorithm called BesSqRootRat to find candidates for $(\nu, f)$ in the rational case, if they exist. An example for this case can be found in our Maple worksheet associated to this thesis.

\subsubsection{Example}

We have written some algorithms (depending on many cases) on how to find Bessel square root $\check{\mathrm{B}}_{\nu}^{2}=\mathrm{B}_{\nu}^{2}(\sqrt{x})$ type solutions, if they exist, with change of variable parameters which are not square of rational functions in $k(x)$. We have also summarized them by writing another algorithm called BesSqRootSolutions which takes as input a third-order linear differential operator $\mathrm{L}$ and returns, if they exist, all the parameters of transformations $\left(r, r_{2}, r_{1}, r_{0}, f \in k(x)\right)$ and also the Bessel parameter $\nu \in k$ such that we are in the situation

$$
\mathrm{L}_{\check{B}_{\nu}^{2}} \stackrel{f}{\longrightarrow}{ }_{C} \mathrm{M} \longrightarrow \longrightarrow_{E G} \mathrm{~L} .
$$

If not, it will return "No Bessel square roots type solutions". This algorithm deals with all the cases.

We will take here just one example and show explicitly how some of our algorithms work. $5 / 12$.

Let us consider the Bessel square root operator $\mathrm{L}_{B B}=\mathrm{L}_{\breve{B}_{\nu}^{2}}$ with parameter $\nu=$

$>$ LBB: $=\operatorname{subs}(n u=5 / 12, \mathrm{LBB})$;

$$
L B B:=2 x^{2} D x^{3}+6 x D x^{2}+\left(\frac{119}{72}-2 x\right) D x-1
$$
by

Let us apply to $\mathrm{L}_{B B}$ the change of variables transformation with parameter $f$ given

$$
\begin{array}{r}
>f:=((x-1) \sim 5 *(x-7) \sim 4) /((x-12) *(x-14) *(x-3)) ; \\
f:=\frac{(x-1)^{5}(x-7)^{4}}{(x-12)(x-14)(x-3)}
\end{array}
$$

That gives us the following operator called $\mathrm{L}$

$$
\begin{aligned}
> & \mathrm{L}:=\text { ChangeOfVariables }(\mathrm{LBB}, \mathrm{f}) ; \\
L:= & 36(x-1)^{2}(x-7)^{2}\left(8967-5878 x+3 x^{4}-109 x^{3}+1307 x^{2}\right)^{2}(x-12)^{4}(x-3)^{4} \\
& \times(x-14)^{4} D x^{3}+108(x-1)(x-7)\left(-218 x^{7}+6499 x^{6}-104180 x^{5}+987827 x^{4}\right. \\
& \left.+3 x^{8}-5732068 x^{3}+20096501 x^{2}-38751258 x+30858534\right)\left(8967-5878 x+3 x^{4}\right. \\
& \left.-109 x^{3}+1307 x^{2}\right)(x-12)^{3}(x-3)^{3}(x-14)^{3} D x^{2}-(x-12)(x-3)(x-14) \\
& \times\left(18520665120908031123126 x+3998651202636212263 x^{15}+173832480 x^{23}\right. \\
& -9053528544 x^{22}-245979402650313330482820 x^{4}-911870515880074445602 x^{12} \\
& -29483091110607109603 x^{14}-3091611815345853 x^{18}-447919384137869370 x^{16}
\end{aligned}
$$




$$
\begin{aligned}
& -69036545299869074699541 x^{2}-13240379544983023993231 x^{10}+11664 x^{25} \\
& -2080080 x^{24}-87252176473291126851370 x^{8}+280228836976025631929820 x^{5} \\
& +3824107057746349765223 x^{11}+41222620813286268 x^{17}+329691581136 x^{21} \\
& +180075462606323412536 x^{13}-8928362729232 x^{20}+186723184797765 x^{19} \\
& -242129495391633879835802 x^{6}+37650005677084427654216 x^{9} \\
& -2300502610610535723336+157429488642064200020025 x^{3} \\
& \left.+163041223796184338489462 x^{7}\right) D x-144(x-7)^{3}(x-1)^{4}(8967-5878 x \\
& \left.+3 x^{4}-109 x^{3}+1307 x^{2}\right)^{5}
\end{aligned}
$$

Let us assume that we have as input this operator $\mathrm{L}$ and then see how we can find its Bessel square root $\check{\mathrm{B}}_{\nu}^{2}=\mathrm{B}_{\nu}^{2}(\sqrt{x})$ type solutions with our codes if they exist. That means our code should return the Bessel parameter $\nu$ and the transformation parameters such that

$$
\mathrm{L}_{B B} \stackrel{f}{\longrightarrow} C \mathrm{M} \longrightarrow E G \mathrm{~L} .
$$

Let ext be the set of all non-rational numbers, parameters and names (except the variable $x$ ) which appear in the expression of the coefficients of $\mathrm{L}$ :

$>\operatorname{ext}:=\operatorname{indets}(\mathrm{L},\{\operatorname{RootOf}, \mathrm{name}\}) \operatorname{minus}\{\mathrm{x}, \mathrm{Dx}\}$;

$$
\text { ext }:=\{\}
$$

Let extp be the set of all algebraic numbers given by the RootOf expressions in ext such that all its elements are algebraic over the minimal field containing $\mathbb{Q} \cup$ (ext $\backslash$ extp). Since sometimes we can use in our computations the RootOf expression associated with its index, we will take extp $=\{\}$.

Let us denote by $\mathrm{E}$ the set of all the singularities of L (we can find it using our implemented code called Singular).

$>\mathrm{E}:=$ Singular $(\mathrm{L}, \mathrm{ext})$ :

Then the set of non-apparent singularities of $\mathrm{L}$ is given by

$>\mathrm{F}:=\operatorname{NotAppSing}(\mathrm{L}, \mathrm{E}$, ext $)$;

$$
F:=[[\infty, \infty],[x-1,1],[x-3,3],[x-7,7],[x-12,12],[x-14,14]]
$$

Let $S_{\text {irr }}$ be the set of irregular singularities of $\mathrm{L}$ which is the set of poles of $f$.

$>\operatorname{Sirr}:=$ irrsingBesSqRoot $(L, t, F, e x t)$;

$$
\begin{aligned}
\operatorname{Sirr}:=[ & {[[\infty, \infty],[x-3,3],[x-12,12],[x-14,14]],\left[\left[3,-6 t^{-3}+8 t^{-2}-78 t^{-1}\right.\right.} \\
& \left.+3,6 t^{-3}-8 t^{-2}+78 t^{-1}+3\right],\left[1 / 2, \frac{64}{33} \frac{\sqrt{22}}{\sqrt{t}}+1 / 2,-\frac{64}{33} \frac{\sqrt{22}}{\sqrt{t}}+1 / 2\right],[1 / 2, \\
& \left.\left.\frac{-\frac{3025}{6} i \sqrt{22}}{\sqrt{t}}+1 / 2, \frac{\frac{3025}{6} i \sqrt{22}}{\sqrt{t}}+1 / 2\right],\left[1 / 2, \frac{8281}{22} \frac{\sqrt{286}}{\sqrt{t}}+1 / 2,-\frac{8281}{22} \frac{\sqrt{286}}{\sqrt{t}}+1 / 2\right]\right], \\
& {\left[\left[-6 t^{-3}+8 t^{-2}-78 t^{-1}\right],\left[-\frac{64}{33} \frac{\sqrt{22}}{\sqrt{t}}\right],\left[\frac{\frac{3025}{6} i \sqrt{22}}{\sqrt{t}}\right],\left[-\frac{8281}{22} \frac{\sqrt{286}}{\sqrt{t}}\right]\right],[3,1 / 2,1 / 2,} \\
& 1 / 2]]
\end{aligned}
$$

Let us take $\mathrm{G}$ as the set of elements of $\mathrm{F}$ at which $\mathrm{L}$ has all its exponent differences in $k$. Let $\mathrm{S}_{\text {reg }}$ be the set of elements of $\mathrm{G}$ at which $\mathrm{L}$ has logarithmic solutions or all its exponent differences are not, at same time, in $\mathbb{N}$. All the elements of $S_{\text {reg }}$ belong to the set of the zeroes of $f$ and they are given by

$$
\begin{aligned}
>\text { Sreg } & :=\text { regsingtrueBesSqRoot (L, t, Sirr }[-1], \text { ext }) ; \\
\text { Sreg } & :=\left[[[x-1,1],[x-7,7]],\left[\left[-\frac{25}{12}, 0, \frac{25}{12}\right],[-5 / 3,0,5 / 3]\right],\left[\left[\frac{25}{12}\right],[5 / 3]\right]\right]
\end{aligned}
$$


$\mathrm{S}_{\text {reg }}$ can be divided into two subsets: the set of non-removable singularities of L (at least one exponent difference is not an integer or $\mathrm{L}$ has logarithmic solution(s)) and a set of removable singularities of $\mathrm{L}$ (all the exponent differences are integers and $\mathrm{L}$ doesn't have logarithmic solution(s)). The set of non-removable singularities of L can also be divided into two sets: the singularities at which $L$ has logarithmic solutions and the singularities at which $\mathrm{L}$ doesn't have logarithmic solutions. Hence, let us denote by $\mathrm{RS}_{\text {reg }}$ a set of three sets: the first is the set of non-removable singularities of $\mathrm{L}$ with non-logarithmic solutions, the second is the set of removable singularities of $\mathrm{L}$ in $\mathrm{S}_{\text {reg }}$, and the third is the set of singularities of $\mathrm{L}$ with logarithmic solutions. With our code called SregseptrueBesSqRoot we can find this set $\mathrm{RS}_{\text {reg: }}$ :

$$
\begin{gathered}
>\text { NRemSreg :=SregseptrueBesSqRoot(L,Sreg,ext) [1]; } \\
\text { NRemSreg := }\left[[[x-1,1],[x-7,7]],\left[\left[-\frac{25}{12}, 0, \frac{25}{12}\right],[-5 / 3,0,5 / 3]\right],\left[\left[\frac{25}{12}\right],[5 / 3]\right]\right] \\
>\text { LogSreg := SregseptrueBesSqRoot (L, Sreg, ext) [3]; } \\
\operatorname{LogSreg}:=[] \\
>\text { RemSreg := SregseptrueBesSqRoot (L, Sreg, ext) [2]; } \\
\text { RemSreg }:=[]
\end{gathered}
$$

Since NRemS $_{\text {reg }} \neq[$ ], we know some zeroes of $f$. But we are not sure that they represent all the zeroes of $f$ because the exponent differences of $\mathrm{L}$ at elements of $\mathrm{NRemS}_{\text {reg }}$ are in $\mathbb{Q} \backslash \mathbb{Z}$.

In order to make our codes faster, we have implemented one procedure called IrrRegAppsingBesSqRoot which gives at the same time and, by avoiding duplicate computations, in a shorter time all the outputs of the procedures irrsingBesSqRoot, regsingtrueBesSqRoot and SregseptrueBesSqRoot.

$>\quad \mathrm{R} 1:=\operatorname{IrrRegAppsingBesSqRoot}(\mathrm{L}, \mathrm{t}, \mathrm{E}, \mathrm{ext})$ :

Let $f=A / B$ with $\mathrm{A}$ and $\mathrm{B}$ as in our theoretical part. Using our code called SirrBesSqRootinfo1, we can compute the truncated series for $f$ related to the elements of $\mathrm{S}_{\text {irr }}$, the degree of $\mathrm{A}$ denoted $d_{\mathrm{A}}$ and also the number $n$ of linear equations satisfied by the coefficients of A. It shows us if we are in the "Easy case" or not. If we are not in this case, according to the exponent differences of elements in $\mathrm{RS}_{\text {reg }}[1]$, we can see in which case we are.

$$
\begin{aligned}
&> \text { info1:= } \\
& \text { info1 }:=\left[\left[\left[\infty, x^{-1},\left[82 x^{4}-4 x^{5}+x^{6}\right], 4,\{\},\{\}\right],\left[3, x-3,\left[8192(99 x-297)^{-1}\right],\right.\right.\right. \\
&1,\{\},\{\}],\left[12, x-12,\left[-100656875(18 x-216)^{-1}\right], 1,\{\},\{\}\right],[14, x-14, \\
& {\left.\left.\left.\left[891474493(22 x-308)^{-1}\right], 1,\{\},\{\}\right]\right], 9,8,(x-12)(x-3)(x-14), 1\right] }
\end{aligned}
$$

We have $n=8$ and $d_{\mathrm{A}}=9$. Hence $n<d_{\mathrm{A}}$ and therefore we cannot solve those equations and get $\mathrm{A}$ : that is not the "Easy case".

According to the exponent differences of the elements of $\mathrm{NRemS}_{\text {reg }}$, we are here in the rational case. Our code called findBesSqRootRat gives us the set of candidates for $(\nu, f)$.

$>$ findBesSqRootRat (L, R1, info1, $x, t, T$, ext) ;

$$
\left\{\left[\left[\frac{5}{12}\right], \frac{(x-1)^{5}(x-7)^{4}}{(x-12)(x-3)(x-14)}\right]\right\}
$$


All the transformation parameters, if they exist, and also the Bessel parameter can be given using our code called BesSqRootSolutions :

$>$ BesSqRootSolutions(L);

$$
\left\{\left[\frac{5}{12},[0],[1], \frac{(-1+x)^{5}(x-7)^{4}}{(x-12)(x-14)(x-3)}\right]\right\}
$$

Hence, L has $\check{\mathrm{B}}_{\nu}^{2}=\mathrm{B}_{\nu}^{2}(\sqrt{x})$ type solutions with Bessel parameter $\nu=\frac{5}{12}$ and transformation parameters: $r=0, r_{0}=1, r_{1}=0, r_{2}=0$ and $f=\frac{(x-1)^{5}(x-7)^{4}}{(x-12)(x-14)(x-3)}$.

\subsubsection{Uniqueness of the Change of Variables Parameter $f$}

In this section, we want to show that the change of variables $f$ is unique. We prove it only for $k(x)=\overline{\mathbb{Q}}(x)$ and $k(x)=\overline{\mathbb{Q}(x)}$ and $\nu \in \overline{\mathbb{Q}}$. We also exclude the case $\nu=1 / 2+n, n \in \mathbb{Z}$, because $\mathrm{L}_{\check{B}_{\nu}^{2}}$ will be reducible.

All the theorems, lemmas and remarks here about the Bessel square root operators $\mathrm{L}_{\check{B}_{\nu}^{2}}$ are similar as in the $\mathrm{PhD}$ thesis of Quan Yuan (see [42]) about Bessel operators related with second order linear differential operators in the part called: Proof of Uniqueness.

Let us consider

$$
\mathrm{L}_{\check{B}_{\nu}^{2}} \stackrel{f}{\longrightarrow} C \mathrm{M}_{1}{\stackrel{r_{0}, r_{1}, r_{2}}{\longrightarrow}}_{G} \mathrm{M}_{2} \stackrel{r}{\longrightarrow}{ }_{E} \mathrm{~L} \text {. }
$$

Lemma 4.50. Let $r, r_{0} \in \overline{\mathbb{Q}(x)}, \nu \in \overline{\mathbb{Q}}, f=x, r_{2}=0, r_{1}=0$, and $\exp \left(\int r d x\right) r_{0}$ is a constant. Then $\exp \left(\int r d x\right) \in \overline{\mathbb{Q}(x)}$ and we can rewrite

$$
\exp \left(\int r d x\right)\left[r_{0} \mathrm{~B}_{\nu}^{2}(\sqrt{f})+r_{1}\left(\mathrm{~B}_{\nu}^{2}(\sqrt{f})\right)^{\prime}+r_{2}\left(\mathrm{~B}_{\nu}^{2}(\sqrt{f})\right)^{\prime \prime}\right]
$$

as

$$
\widetilde{r}_{0} \mathrm{~B}_{\nu}^{2}(\sqrt{f})+\widetilde{r}_{1}\left(\mathrm{~B}_{\nu}^{2}(\sqrt{f})\right)^{\prime}+\widetilde{r}_{2}\left(\mathrm{~B}_{\nu}^{2}(\sqrt{f})\right)^{\prime \prime}, \quad \widetilde{r}_{0}, \widetilde{r}_{1}, \widetilde{r}_{2} \in \overline{\mathbb{Q}(x)}
$$

Proof. It is similar as the proof of Lemma 21 in [42].

By taking $\mathrm{L}$ as $\mathrm{L}_{\breve{B}_{\nu}^{2}}$ we have the following lemma:

Lemma 4.51. If $\nu \in \overline{\mathbb{Q}}$, and $r_{0} \mathrm{~B}_{\nu}^{2}(\sqrt{f})+r_{1}\left(\mathrm{~B}_{\nu}^{2}(\sqrt{f})\right)^{\prime}+r_{2}\left(\mathrm{~B}_{\nu}^{2}(\sqrt{f})\right)^{\prime \prime}$ is a solution of $\mathrm{L}_{\check{B}_{\nu}^{2}}$ where $r_{0}, r_{1}, r_{2}, f \in \overline{\mathbb{Q}(x)}$, then $f= \pm x+c$, where $c$ is a constant.

Proof. It is similar as the proof of Lemma 25 in [42].

Lemma 4.52. The constant $c$ in Lemma 4.51 is equal to 0.

Proof. It is similar as the proof of Lemma 26 in [42].

By taking $\mathrm{L}$ as $\mathrm{L}_{\check{B}_{\nu}^{2}}$ we have the following theorem:

Theorem 4.53. If $\mathrm{L}_{\check{B}_{\nu}^{2}}$ has a solution

$$
\exp \left(\int r d x\right)\left[r_{0} \mathrm{~B}_{\nu}^{2}(\sqrt{f})+r_{1}\left(\mathrm{~B}_{\nu}^{2}(\sqrt{f})\right)^{\prime}+r_{2}\left(\mathrm{~B}_{\nu}^{2}(\sqrt{f})\right)^{\prime \prime}\right]
$$

where $r, r_{0}, r_{1}, r_{2}, f \in \overline{\mathbb{Q}(x)}$ and $\nu \in \overline{\mathbb{Q}}$, then $f=x, r_{2}=0, r_{1}=0$ and $\exp \left(\int r d x\right) r_{0}$ is a constant. 
Proof. It is similar as the proof of Theorem 11 in [42]. We use Lemma 4.50, Lemma 4.51 and Lemma 4.52.

Remark 4.54. Using a standard argument, Theorem 4.53 implies a similar statement with $\mathbb{Q}$ replaced by $\mathbb{C}$.

Theorem 4.55. (Uniqueness)

If $\mathrm{L}$ has solutions

$$
\begin{array}{r}
\exp \left(\int r d x\right)\left[r_{0} \mathrm{~B}_{\nu}^{2}\left(\sqrt{f_{1}}\right)+r_{1}\left(\mathrm{~B}_{\nu}^{2}\left(\sqrt{f_{1}}\right)\right)^{\prime}+r_{2}\left(\mathrm{~B}_{\nu}^{2}\left(\sqrt{f_{1}}\right)\right)^{\prime \prime}\right], \\
\text { and } \exp \left(\int r d x\right)\left[\widetilde{r}_{0} \mathrm{~B}_{\nu}^{2}\left(\sqrt{f_{2}}\right)+\widetilde{r}_{1}\left(\mathrm{~B}_{\nu}^{2}\left(\sqrt{f_{2}}\right)\right)^{\prime}+\widetilde{r}_{2}\left(\mathrm{~B}_{\nu}^{2}\left(\sqrt{f_{2}}\right)\right)^{\prime \prime}\right]
\end{array}
$$

where $r, r_{0}, r_{1}, r_{2}, \widetilde{r}_{0}, \widetilde{r}_{1}, \widetilde{r}_{2}, f_{1}, f_{2} \in \overline{\mathbb{Q}}(x)$, then $f_{1}= \pm f_{2}$

Proof. It is similar as the proof of Theorem 12 in [42] which takes, for $i \in\{1,2\} f_{i}=$ $\frac{F_{i}}{D_{i}} \in \overline{\mathbb{Q}}(x)$, where $F_{i}, D_{i} \in \overline{\mathbb{Q}}[x]$, and defines an inverse (denoted $f_{i}^{-1}$ ) of $f_{i}$ over $\overline{\mathbb{Q}(x)}$ as a solution in $\overline{\mathbb{Q}(x)}$ of $F_{i}(T)-D_{i}(T) \cdot x \in \overline{\mathbb{Q}}(x)[T]$ with unknown $T$.

Remark 4.56. This Theorem 4.55 also holds if we replace $\overline{\mathbb{Q}}(x)$ by $\overline{\mathbb{Q}(x)}$. The proof is essentially the same, but, for $i \in\{1,2\}$, the definition of $f_{i}^{-1}$ is more technical (similar as for Remark 23 in (42]).

Remark 4.57. Let $\mathbb{Q} \subseteq k \subseteq \overline{\mathbb{Q}}$. Let $\mathrm{K}=k(x)$ and $\mathrm{K}[\partial]$ of order 3. Suppose L has a solution of the form

$$
\exp \left(\int r d x\right)\left[r_{0} \mathrm{~B}_{\nu}^{2}(\sqrt{f})+r_{1}\left(\mathrm{~B}_{\nu}^{2}(\sqrt{f})\right)^{\prime}+r_{2}\left(\mathrm{~B}_{\nu}^{2}(\sqrt{f})\right)^{\prime \prime}\right],
$$

where $r, r_{0}, r_{1}, r_{2}, f \in \overline{\mathbb{Q}(x)}$. The uniqueness of $f$ implies $f \in \mathrm{K}$. In our algorithm any candidate $g$ for $f$ that is not defined over $\mathrm{K}$ can thus be discarded without further computation.

\subsection{Algebraic Extension}

This section is similar as the PhD thesis of Quan Yuan (see [42]) in the part called: Algebraic Extension.

There are three types of algebraic extensions we need to deal with:

1- When a singularity or the Bessel parameter $\nu$ is not in $k$.

If $\nu \notin k$, we are in the irrational case and we just use the coefficients of irrational parts of the exponent differences of $\mathrm{L}$ at all the elements of $\mathrm{S}_{\mathrm{reg}}(\mathrm{L})$ (those irrational parts are the same: the irrational part of $\nu$ ) to find the multiplicity orders of the zeroes of $f$. Hence we can find $f$. If a singularity $s$ is not in $k$, by using Lemma 4.22 and Lemma 4.24, we can still get the linear equations we need.

2- When $k \neq \mathbb{Q}$.

There is no problem because our algorithm works for all $k$ of characteristic 0 . But we might need more computations in the rational case. 
3- When the algebraic extension is introduced by the algorithm itself.

It happens when $c$ is not unique (up to multiplication by a $d$-th power) over $k$. Although it is possible to compute all c's up to $\sim$ directly, it is a complicated algebraic problem. Instead, to find proper $c$ 's in that case, we add one irregular singularity $s$ into $k$. So now we work over $k(s)$. This action requires that we compute the exponent differences over the new field $k(s)$, because some algebraic singularities might factor now. Once we extend the base field, we only need to deal with the other two types of algebraic extensions. And $c$ is unique (up to multiplication by a $d$-th power) in the new field according to Lemma 4.45. 


\section{Chapter 5}

\section{${ }_{2} F_{2},{ }_{1} F_{2},{ }_{0} F_{2}$ and ${ }_{1} F_{1}^{2}$ Type Solutions}

All the results in this chapter are new.

We take here the initial conditions as for the Bessel square type solutions in the last chapter. We just change the Bessel square functions (see the first part of the last chapter) by the functions ${ }_{2} F_{2},{ }_{1} F_{2},{ }_{0} F_{2},{ }_{1} F_{1}^{2}$. Hence we will get complete solver algorithms for ${ }_{2} F_{2}$, ${ }_{1} F_{2},{ }_{0} F_{2}$ and ${ }_{1} F_{1}^{2}$ type solutions.

Let $\mathrm{L} \in \mathrm{K}[\partial]$ be our input operator which has to be an irreducible third-order linear differential operator. Let $\mathrm{L}_{0}$ be the operator associated to the function $F_{0} \in$ $\left\{{ }_{2} F_{2},{ }_{1} F_{2},{ }_{0} F_{2},{ }_{1} F_{1}^{2}\right\}$. We just have to consider (see chapter three)

$$
\mathrm{L}_{0} \stackrel{f}{\longrightarrow}_{C} \mathrm{M} \longrightarrow \longrightarrow_{E G} \mathrm{~L} \text {. }
$$

with $\mathrm{M} \in \mathrm{K}[\partial]$.

As for the Bessel square case, we will just take a closer look at the part $\mathrm{L}_{0} \stackrel{f}{\longrightarrow}{ }_{C} \mathrm{M}$. Once we found the parameters of the function $F_{0}$ and the parameter $f$, we can obtain M from $\mathrm{L}_{0}$. Then, by solving the question of equivalence between $\mathrm{M}$ and $\mathrm{L}$, we can finally solve (5.1).

For $n \in \mathbb{N} \backslash\{0\}$ and $a$ an element of $\mathrm{K}=k(x)$ or not, when we will talk about $a$ modulo $\frac{1}{n} \mathbb{Z}$, that will means a modulo an additive element of $\frac{1}{n} \mathbb{Z}$.

\subsection{Some Help Tools}

\subsubsection{Properties}

We recall here some properties about hypergeometric functions. Theorem 5.1, Theorem 5.2 and Definition 5.4 are from [25].

Theorem 5.1. Let $\theta$ be the differential operator given by $\theta(f(x))=x f^{\prime}(x)=x \frac{d}{d x} f(x)$. The generalized hypergeometric function ${ }_{p} F_{q}\left(\begin{array}{c}a_{1}, \ldots, a_{p} \\ b_{1}, \ldots, b_{q}\end{array} \mid x\right)$ satisfies the derivative rule

$$
\theta\left(f_{n}(x)\right)=n\left(f_{n+1}(x)-f_{n}(x)\right)
$$

for any of its numerator parameters $n:=a_{i}(i=1, \ldots, p)$, and

$$
\theta\left(f_{n}(x)\right)=(n-1)\left(f_{n-1}(x)-f_{n}(x)\right)
$$


for any of its denominator parameters $n:=b_{i}(i=1, \ldots, q)$.

Proof. See [25].

Theorem 5.2. The generalized hypergeometric function ${ }_{p} F_{q}\left(\begin{array}{c}a_{1}, \ldots, a_{p} \\ b_{1}, \ldots, b_{q}\end{array} \mid x\right)$ has this property:

$$
\frac{d}{d x}{ }_{p} F_{q}\left(\begin{array}{c}
a_{1}, \ldots, a_{p} \\
b_{1}, \ldots, b_{q}
\end{array} \mid x\right)=\frac{\prod_{i=1}^{p} a_{i}}{\prod_{i=1}^{p} b_{i}} F_{q}\left(\begin{array}{c}
a_{1}+1, \ldots, a_{p}+1 \\
b_{1}+1, \ldots, b_{q}+1
\end{array} \mid x\right)
$$

Proof. See [25].

Corollary 5.3. The linear space over $\mathbb{C}(x)$ spanned by

$$
{ }_{p} F_{q}\left(\begin{array}{c}
a_{1}, \ldots, a_{p} \\
b_{1}, \ldots, b_{q}
\end{array} \mid x\right) \text { and } x \frac{d}{d x}{ }_{p} F_{q}\left(\begin{array}{c}
a_{1}, \ldots, a_{p} \\
b_{1}, \ldots, b_{q}
\end{array} \mid x\right)
$$

contains each of ${ }_{p} F_{q}\left(\begin{array}{c}a_{1}, \ldots, a_{j}+1, \ldots, a_{p} \mid \\ b_{1}, \ldots, b_{q}\end{array} \mid x\right),{ }_{p} F_{q}\left(\begin{array}{c}a_{1}, \ldots, a_{p} \\ b_{1}, \ldots, b_{j}-1, \ldots, b_{q}\end{array} \mid x\right)$,

$$
x_{p} F_{q}\left(\begin{array}{c}
a_{1}+1, \ldots, a_{p}+1 \\
b_{1}+1, \ldots, b_{q}+1
\end{array} \mid x\right) \text { and }{ }_{p} F_{q}\left(\begin{array}{c}
a_{1}, \ldots, a_{p} \\
b_{1}, \ldots, b_{q}
\end{array} \mid x\right) \text {. }
$$

Similarly, by applying the differentiation formulas twice, there are $\left(\begin{array}{c}p+q+3 \\ 2\end{array}\right)$ such functions contained in $\left\{1, x \frac{d}{d x},\left(x \frac{d}{d x}\right)^{2}\right\}{ }_{p} F_{q}\left(\begin{array}{c}a_{1}, \ldots, a_{p} \\ b_{1}, \ldots, b_{q}\end{array} \mid x\right)$, which has dimension three (any four are linearly dependent).

Proof. We use Theorem 5.1 and Theorem 5.2.

Definition 5.4. A function obtained by adding \pm 1 to exactly one of the parameters $a_{i}$ in $b_{j}(i \in\{1, \ldots, p\}$ and $j \in\{1, \ldots, q\})$ is called contiguous to ${ }_{p} F_{q}\left(\begin{array}{c}a_{1}, \ldots, a_{p} \\ b_{1}, \ldots, b_{q}\end{array} \mid x\right)$.

For a given ${ }_{p} F_{q}$ hypergeometric function, the following corollary shows us the existence of a linear space over $\mathbb{C}(x)$, with dimension equals to the order of the differential equation for ${ }_{p} F_{q}$, which contains all the functions coming from ${ }_{p} F_{q}$ and all its derivatives by any integer parameter-shift. 
Corollary 5.5. Let us consider a generalized hypergeometric function ${ }_{p} F_{q}\left(\begin{array}{c}a_{1}, \ldots, a_{p} \\ b_{1}, \ldots, b_{q}\end{array} \mid x\right)$ and the $\mathbb{C}(x)$ vector space

$$
\mathrm{S}:=\mathbb{C}(x)_{p} F_{q}\left(\begin{array}{c}
a_{1}, \ldots, a_{p} \\
b_{1}, \ldots, b_{q}
\end{array} \mid x\right)+\cdots+\mathbb{C}(x) \frac{d^{n-1}}{d x^{n-1}} p F_{q}\left(\begin{array}{c}
a_{1}, \ldots, a_{p} \\
b_{1}, \ldots, b_{q}
\end{array} \mid x\right)
$$

where $n=\max \{p, q+1\}$ is the order of the differential equation for ${ }_{p} F_{q}\left(\begin{array}{c}a_{1}, \ldots, a_{p} \\ b_{1}, \ldots, b_{q}\end{array} \mid x\right)$.

Hence, $\mathrm{S}$ is invariant under substitution $\nu \rightarrow \nu+1$ and $\nu \rightarrow \nu-1$ of upper and lower $\operatorname{parameter}(s)$ of $F_{q}\left(\begin{array}{c|c}a_{1}, \ldots, a_{p} \\ b_{1}, \ldots, b_{q}\end{array} \mid x\right)$.

Proof. We use Theorem 5.1 and Theorem 5.2.

Remark 5.6. In our case, when ${ }_{p} F_{q}$ satisfies a third-order differential equation, we have $n=\max \{p, q+1\}=3$ and

$\mathrm{S}:=\mathbb{C}(x)_{p} F_{q}\left(\begin{array}{c}a_{1}, \ldots, a_{p} \\ b_{1}, \ldots, b_{q}\end{array} \mid x\right)+\mathbb{C}(x) \frac{d}{d x}{ }_{p} F_{q}\left(\begin{array}{c}a_{1}, \ldots, a_{p} \\ b_{1}, \ldots, b_{q}\end{array} \mid x\right)+\mathbb{C}(x) \frac{d^{2}}{d x^{2}}{ }^{2} F_{q}\left(\begin{array}{c}a_{1}, \ldots, a_{p} \\ b_{1}, \ldots, b_{q}\end{array} \mid x\right)$.

Hence, this space $\mathrm{S}$ is invariant under any integer parameter-shift of ${ }_{p} F_{q}$. Therefore, we will assume that the upper and lower parameter $(s)$ of ${ }_{p} F_{q}$ belong to $[-1,1]$.

Notation: In the rest of this thesis, the function $F_{0}$ refers to any element of the set $\left\{{ }_{2} F_{2},{ }_{1} F_{2},{ }_{0} F_{2},{ }_{1} F_{1}^{2}\right\}$ with

1. $a_{1}$ and $a_{2}$ as upper parameters when $F_{0}={ }_{2} F_{2}$,

2. $a_{1}$ as upper parameter when $F_{0} \in\left\{{ }_{1} F_{2},{ }_{1} F_{1}^{2}\right\}$

3. $b_{1}$ and $b_{2}$ as lower parameters when $F_{0} \in\left\{{ }_{2} F_{2},{ }_{1} F_{2},{ }_{0} F_{2}\right\}$,

4. $b_{1}$ as lower parameter when $F_{0}={ }_{1} F_{1}^{2}$.

Lemma 5.7. Consider (5.1) and $F_{0} \in\left\{{ }_{2} F_{2},{ }_{1} F_{2},{ }_{0} F_{2},{ }_{1} F_{1}^{2}\right\}$. If $\mathrm{S}_{\text {reg }}(\mathrm{L}) \neq \emptyset$ then the following statements are equivalent:

(a)- The lower parameter(s) of $F_{0}$ satisfy

* $b_{1} \in \mathbb{Z}$ or $b_{2} \in \mathbb{Z}$ or $b_{1}-b_{2} \in \mathbb{Z}$ or $\left(2 b_{1}, 2 b_{2} \in \mathbb{Z}\right.$ with $\left.b 1 \times b 2<0\right)$ for $F_{0} \in$ $\left\{{ }_{2} F_{2},{ }_{1} F_{2},{ }_{0} F_{2}\right\}$, and

* $b_{1} \in \mathbb{Z}$ for $F_{0}={ }_{1} F_{1}^{2}$

(b)- There is $p_{0} \in \mathrm{S}_{\text {reg }}(\mathrm{L})$ such that $\mathrm{L}$ is logarithmic at $p_{0}$. 
Proof. Similar as the proof of Lemma 4.8: we have to use Remark 5.6 and Remark 3.4.

For computing $f$ and the upper and lower parameter(s) of $F_{0} \in\left\{{ }_{2} F_{2},{ }_{1} F_{2},{ }_{0} F_{2},{ }_{1} F_{1}^{2}\right\}$, the only information retrieved from $L$ that we can use is the information on invariance of exponent differences of $\mathrm{L}$ under projective equivalence. The poles and zeroes of $f$ are the main points at which we can really use those exponent differences. Let us check the exponent differences of $\mathrm{L}$ at those points.

\subsection{Exponent Differences}

Let $\mathrm{L}_{0} \in\left\{\mathrm{L}_{B_{\nu}^{2}}, \mathrm{~L}_{22}, \mathrm{~L}_{12}, \mathrm{~L}_{02}, \mathrm{~L}_{11}^{2}\right\} . \mathrm{L}_{0}$ has two singularities: one regular at $x=0$ and the other irregular at $x=\infty$. The generalized exponents of $\mathrm{L}_{0}$ at 0 and $\infty$ are

- For $\mathrm{L}_{0}=\mathrm{L}_{22}$

$$
\begin{aligned}
& >\quad \text { gen_exp(L22, } \mathrm{t}, \mathrm{x}=0) \\
& {[[0, t=x],[1-b 1, t=x],[1-b 2, t=x]]} \\
& >\quad \operatorname{gen} \_\exp (\mathrm{L} 22, \mathrm{t}, \mathrm{x}=\text { infinity) } \\
& \quad\left[\left[a 1, t=x^{-1}\right],\left[a 2, t=x^{-1}\right],\left[-t^{-1}+b 1-a 1-a 2+b 2, t=x^{-1}\right]\right]
\end{aligned}
$$

- For $\mathrm{L}_{0}=\mathrm{L}_{12}$

$>\quad$ gen_exp $(\mathrm{L} 12, \mathrm{t}, \mathrm{x}=0)$;

$$
\begin{gathered}
{[[0, t=x],[1-b 1, t=x],[1-b 2, t=x]]} \\
>\quad \operatorname{gen} \_\exp (\mathrm{L} 12, \mathrm{t}, \mathrm{x}=\text { infinity }) ; \\
{\left[\left[a 1, t=x^{-1}\right],\left[t^{-1}+1 / 2 b 1-1 / 4-1 / 2 a 1+1 / 2 b 2, t^{2}=x^{-1}\right]\right]}
\end{gathered}
$$

- For $\mathrm{L}_{0}=\mathrm{L}_{02}$

$$
\begin{aligned}
& >\quad \operatorname{gen} \_\exp (\mathrm{L} 02, \mathrm{t}, \mathrm{x}=0) \\
& {[[0, t=x],[1-b 1, t=x],[1-b 2, t=x]]} \\
& >\quad \operatorname{gen} \_\exp (\mathrm{L} 02, \mathrm{t}, \mathrm{x}=\text { inf inity }) ; \\
& {\left[\left[t^{-1}-1 / 3+1 / 3 b 2+1 / 3 b 1,-t^{3}=x^{-1}\right]\right]}
\end{aligned}
$$

- For $\mathrm{L}_{0}=\mathrm{L}_{11}^{2}$

$$
\begin{aligned}
& >\quad \operatorname{gen} \_\exp (\mathrm{L} 112, \mathrm{t}, \mathrm{x}=0) ; \\
& \quad[[0, t=x],[1-b 1, t=x],[2-2 b 1, t=x]] \\
& >\quad \operatorname{gen} \_\exp (\mathrm{L} 112, \mathrm{t}, \mathrm{x}=\text { inf inity }) ; \\
& \quad\left[\left[2 a 1, t=x^{-1}\right],\left[-2 t^{-1}-2 a 1+2 b 1, t=x^{-1}\right],\left[-t^{-1}+b 1, t=x^{-1}\right]\right]
\end{aligned}
$$

Hence the ramification index of

- $\mathrm{L}_{22}$ at $x=0$ and $x=\infty$ is 1 ,

- $\mathrm{L}_{12}$ at $x=0$ is 1 and at $x=\infty$ is 2 ,

- $\mathrm{L}_{02}$ at $x=0$ is 1 and at $x=\infty$ is 3 ,

- $\mathrm{L}_{11}^{2}$ at $x=0$ and $x=\infty$ is 1 . 
Using the assumptions of chapter 3, the generalized exponents of $\mathrm{M}$ at

- a zero $p$ of $f$

* for $\mathrm{L}_{0} \in\left\{\mathrm{L}_{22}, \mathrm{~L}_{12}, \mathrm{~L}_{02}\right\}$, are

$$
\left[0, m_{p}\left(1-b_{1}\right), 2 m_{p}\left(1-b_{2}\right)\right],
$$

* for $\mathrm{L}_{0}=\mathrm{L}_{11}^{2}$, are

$$
\left[0, \quad m_{p}\left(1-b_{1}\right), 2 m_{p}\left(1-b_{1}\right)\right],
$$

where $m_{p}$ is the multiplicity order of $p$ as a zero of $f$;

- a pole $p$ of $f$

* For $\mathrm{L}_{0}=\mathrm{L}_{22}$

$$
\left[m_{p} a_{1}, \quad m_{p} a_{2}, \quad m_{p}\left[b_{1}+b_{2}-\left(a_{1}+a_{2}\right)\right]+\sum_{j=-m_{p}}^{-1} j f_{j} t_{p}^{j}\right],
$$

* For $\mathrm{L}_{0}=\mathrm{L}_{12}$

$$
\left[m_{p} a_{1}, \frac{m_{p}}{2}\left(b_{1}+b_{2}-a_{1}-\frac{1}{2}\right)+\sum_{j=-m_{p}}^{-1} \frac{j}{\varepsilon} \bar{f}_{1, j+m_{p}} t_{p}^{j / 2}\right],
$$

with $\varepsilon$ solution of $X^{2}-1=0$ and $f^{1 / 2}=\sum_{j=-m_{p}}^{+\infty} \bar{f}_{1, j+m_{p}} t_{p}^{j / 2}$ with $\bar{f}_{i, j+m_{p}} \in k$,

* For $\mathrm{L}_{0}=\mathrm{L}_{02}$

$$
\left[\frac{m_{p}}{3}\left(b_{1}+b_{2}-1\right)+\sum_{j=-m_{p}}^{-1} \frac{j}{\varepsilon} \bar{f}_{1, j+m_{p}} t_{p}^{j / 3}\right]
$$

with $\varepsilon$ solution of $X^{3}+1=0$ and $f^{1 / 3}=\sum_{j=-m_{p}}^{+\infty} \bar{f}_{1, j+m_{p}} t_{p}^{j / 3}$ with $\bar{f}_{1, j+m_{p}} \in k$,

* For $\mathrm{L}_{0}=\mathrm{L}_{11}^{2}$

$$
\left[2 m_{p} a_{1}, \quad-2 m_{p}\left(a_{1}-b_{1}\right)+2 \sum_{j=-m_{p}}^{-1} j f_{j} t_{p}^{j}, \quad m_{p} b_{1}+\sum_{j=-m_{p}}^{-1} j f_{j} t_{p}^{j}\right],
$$

where $m_{p}$ is the multiplicity order of $p$ as a pole of $f$, and $f=t_{p}^{-m_{p}} \sum_{j=0}^{+\infty} f_{j-m_{p}} t_{p}^{j}$ with $f_{j-m_{p}} \in k$ and $f_{-m_{p}} \neq 0$.

Hence the ramification index of $\mathrm{M}$

- for $\mathrm{L}_{0}=\mathrm{L}_{22}$, at all the zeroes and poles of $f$ is 1 ;

- for $\mathrm{L}_{0}=\mathrm{L}_{12}$, at all the zeroes of $f$ is 1 and at all the poles of $f$ belongs to $\{1,2\}$; 
- for $\mathrm{L}_{0}=\mathrm{L}_{02}$, at all the zeroes of $f$ is 1 and at all the poles of $f$ belongs to $\{1,3\}$;

- for $\mathrm{L}_{0}=\mathrm{L}_{11}^{2}$, at all the zeroes and poles of $f$ is 1 .

Since the exp-product and gauge transformation don't change the ramification index, for a given point $p$, the index of $\mathrm{L}$ at this point is the same like the ramification index of $\mathrm{M}$ also at this point.

Using the generalized exponents of $\mathrm{M}$ at $p$, we can get the exponent differences of $\mathrm{M}$ at $p$. They are given by

- $p$ is a zero of $f$ and

$$
\begin{aligned}
& * \mathrm{~L}_{0} \in\left\{\mathrm{L}_{22}, \mathrm{~L}_{12}, \mathrm{~L}_{02}\right\} \\
& \\
& \left.\qquad m_{p}\left(1-b_{1}\right), m_{p}\left(1-b_{2}\right), m_{p}\left(b_{1}-b_{2}\right)\right], \\
& * \mathrm{~L}_{0}=\mathrm{L}_{11}^{2} \quad\left[m_{p}\left(1-b_{1}\right), 2 m_{p}\left(1-b_{1}\right), m_{p}\left(1-b_{1}\right)\right] .
\end{aligned}
$$

- $p$ is a pole of $f$

${ }^{*} \mathrm{~L}_{0}=\mathrm{L}_{22}$

$$
\begin{aligned}
{\left[m_{p}\left(a_{2}-a_{1}\right),\right.} & m_{p}\left[b_{1}+b_{2}-\left(2 a_{1}+a_{2}\right)\right]+\sum_{j=-m_{p}}^{-1} j f_{j} t_{p}^{j}, \\
& \left.m_{p}\left[b_{1}+b_{2}-\left(a_{1}+2 a_{2}\right)\right]+\sum_{j=-m_{p}}^{-1} j f_{j} t_{p}^{j}\right],
\end{aligned}
$$

$* \mathrm{~L}_{0}=\mathrm{L}_{12}$

$$
\begin{array}{r}
{\left[\frac{m_{p}}{2}\left(b_{1}+b_{2}-3 a_{1}-\frac{1}{2}\right)+\sum_{j=-m_{p}}^{-1} \frac{j}{\varepsilon_{1}} \bar{f}_{1, j+m_{p}} t_{p}^{j / 2}, \quad \frac{m_{p}}{2}\left(b_{1}+b_{2}-3 a_{1}-\frac{1}{2}\right)\right.} \\
\left.+\sum_{j=-m_{p}}^{-1} \frac{j}{\varepsilon_{2}} \bar{f}_{1, j+m_{p}} t_{p}^{j / 2}, \sum_{j=-m_{p}}^{-1} j\left(\varepsilon_{2}^{-1}-\varepsilon_{1}^{-1}\right) \bar{f}_{1, j+m_{p}} t_{p}^{j / 2}\right]
\end{array}
$$

where $\varepsilon_{1}, \varepsilon_{2}$ are the two distinct solutions of $X^{2}-1=0$. Since $\varepsilon_{2}^{-1}-\varepsilon_{1}^{-1}= \pm 2$ then we have

$$
\begin{aligned}
{\left[\frac{m_{p}}{2}\left(b_{1}+b_{2}-3 a_{1}-\frac{1}{2}\right)\right.} & +\sum_{j=-m_{p}}^{-1} \frac{j}{\varepsilon_{1}} \bar{f}_{1, j+m_{p}} t_{p}^{j / 2}, \quad \frac{m_{p}}{2}\left(b_{1}+b_{2}-3 a_{1}-\frac{1}{2}\right) \\
& \left.+\sum_{j=-m_{p}}^{-1} \frac{j}{\varepsilon_{2}} \bar{f}_{1, j+m_{p}} t_{p}^{j / 2}, \pm \sum_{j=-m_{p}}^{-1} 2 j \bar{f}_{1, j+m_{p}} t_{p}^{j / 2}\right]
\end{aligned}
$$


* $\mathrm{L}_{0}=\mathrm{L}_{02}$

$$
\begin{aligned}
& {\left[\sum_{j=-m_{p}}^{-1} j\left(\varepsilon_{2}^{-1}-\varepsilon_{1}^{-1}\right) \bar{f}_{1, j+m_{p}} t_{p}^{j / 3}, \quad \sum_{j=-m_{p}}^{-1} j\left(\varepsilon_{3}^{-1}-\varepsilon_{1}^{-1}\right) \bar{f}_{1, j+m_{p}} t_{p}^{j / 3},\right.} \\
& \left.\sum_{j=-m_{p}}^{-1} j\left(\varepsilon_{3}^{-1}-\varepsilon_{2}^{-1}\right) \bar{f}_{1, j+m_{p}} t_{p}^{j / 3}\right]
\end{aligned}
$$

where $\varepsilon_{1}, \varepsilon_{2}$ and $\varepsilon_{3}$ are the three distinct solutions of $X^{3}+1=0$.

$* \mathrm{~L}_{0}=\mathrm{L}_{11}^{2}$

$$
\begin{array}{r}
2 m_{p}\left(b_{1}-2 a_{1}\right)+2 \sum_{j=-m_{p}}^{-1} j f_{j} t_{p}^{j}, \quad m_{p}\left(b_{1}-2 a_{1}\right)+\sum_{j=-m_{p}}^{-1} j f_{j} t_{p}^{j}, \\
\left.-m_{p}\left(b_{1}-2 a_{1}\right)-\sum_{j=-m_{p}}^{-1} j f_{j} t_{p}^{j}\right] .
\end{array}
$$

Since by the fact that the exponent difference at any point $p$ is invariant modulo $\frac{1}{n_{p}} \mathbb{Z}\left(n_{p}\right.$ is the ramification index of $\mathrm{M}$ at $p$, here $\left.n_{p} \in\{1,2,3\}\right)$ under the exp-product and gauge transformations, the exponent differences of $\mathrm{L}$ at

- a zero $p$ of $f$, for $\mathrm{L}_{0} \in\left\{\mathrm{L}_{22}, \mathrm{~L}_{12}, \mathrm{~L}_{02}\right\}$ are

$$
\left[m_{p}\left(1-b_{1}\right)+\alpha_{1}, \quad m_{p}\left(1-b_{2}\right)+\alpha_{2}, \quad m_{p}\left(b_{1}-b_{2}\right)+\alpha_{3}\right]
$$

with $\alpha_{1}, \alpha_{2}, \alpha_{3} \in \mathbb{Z}$.

- a pole $p$ of $f$

$* \mathrm{~L}_{0}=\mathrm{L}_{22}$

$$
\begin{aligned}
{\left[m_{p}\left(a_{2}-a_{1}\right)+\beta_{1},\right.} & m_{p}\left[b_{1}+b_{2}-\left(2 a_{1}+a_{2}\right)\right]+\sum_{j=-m_{p}}^{-1} j f_{j} t_{p}^{j}+\beta_{2}, \\
& \left.m_{p}\left[b_{1}+b_{2}-\left(a_{1}+2 a_{2}\right)\right]+\sum_{j=-m_{p}}^{-1} j f_{j} t_{p}^{j}+\beta_{3}\right],
\end{aligned}
$$

where $\beta_{1}, \beta_{2}, \beta_{3} \in \mathbb{Z}$.

$* \mathrm{~L}_{0}=\mathrm{L}_{12}$

$$
\begin{aligned}
& {\left[\frac{m_{p}}{2}\left(b_{1}+b_{2}-3 a_{1}-\frac{1}{2}\right)+\sum_{j=-m_{p}}^{-1} \frac{j}{\varepsilon_{1}} \bar{f}_{1, j+m_{p}} t_{p}^{j / 2}+\beta_{1}, \quad \frac{m_{p}}{2}\left(b_{1}+b_{2}\right.\right.} \\
& \left.\left.-3 a_{1}-\frac{1}{2}\right)+\sum_{j=-m_{p}}^{-1} \frac{j}{\varepsilon_{2}} \bar{f}_{1, j+m_{p}} t_{p}^{j / 2}+\beta_{2}, \quad \pm \sum_{j=-m_{p}}^{-1} 2 j \bar{f}_{1, j+m_{p}} t_{p}^{j / 2}+\beta_{3}\right],
\end{aligned}
$$

where $\varepsilon_{1}, \varepsilon_{2}$ are the two distinct solutions of $X^{2}-1=0$ and $\beta_{1}, \beta_{2}, \beta_{3} \in \mathbb{Z}$. 
$* \mathrm{~L}_{0}=\mathrm{L}_{02}$

$$
\begin{aligned}
{\left[\sum_{j=-m_{p}}^{-1} j\left(\varepsilon_{2}^{-1}-\varepsilon_{1}^{-1}\right) \bar{f}_{1, j+m_{p}} t_{p}^{j / 3}+\beta_{1},\right.} & \sum_{j=-m_{p}}^{-1} j\left(\varepsilon_{3}^{-1}-\varepsilon_{1}^{-1}\right) \bar{f}_{1, j+m_{p}} t_{p}^{j / 3}+\beta_{2}, \\
& \left.\sum_{j=-m_{p}}^{-1} j\left(\varepsilon_{3}^{-1}-\varepsilon_{2}^{-1}\right) \bar{f}_{1, j+m_{p}} t_{p}^{j / 3}+\beta_{3}\right],
\end{aligned}
$$

where $\varepsilon_{1}, \varepsilon_{2}$ and $\varepsilon_{3}$ are the three distinct solutions of $X^{3}+1=0$ and $\beta_{1}, \beta_{2}, \beta_{3} \in$ $\mathbb{Z}$.

$* \mathrm{~L}_{0}=\mathrm{L}_{11}^{2}$

$$
\begin{array}{r}
2 m_{p}\left(b_{1}-2 a_{1}\right)+2 \sum_{j=-m_{p}}^{-1} j f_{j} t_{p}^{j}+\beta_{1}, \quad m_{p}\left(b_{1}-2 a_{1}\right)+\sum_{j=-m_{p}}^{-1} j f_{j} t_{p}^{j}+\beta_{2}, \\
\left.-m_{p}\left(b_{1}-2 a_{1}\right)-\sum_{j=-m_{p}}^{-1} j f_{j} t_{p}^{j}+\beta_{3}\right],
\end{array}
$$

where $\beta_{1}, \beta_{2}, \beta_{3} \in \mathbb{Z}$.

Let us first start showing how to find the lower parameters of $F_{0} \in\left\{{ }_{2} F_{2},{ }_{1} F_{2},{ }_{0} F_{2},{ }_{1} F_{1}^{2}\right\}$. This can be done by using the exponent differences of $\mathrm{L}$ at elements of $\mathrm{S}_{\text {reg }}(\mathrm{L})$ if $\mathrm{S}_{\text {reg }}(\mathrm{L}) \neq$ $\emptyset$, or by another technique based on the divisors of the multiplicity orders of the zeroes of $f$ (we need in this situation to find first $f$ before searching for those lower parameters).

\subsection{How to find the lower parameters $b_{1}$ and $b_{2}$ or $b$ of $F_{0}$ when $\mathrm{S}_{\text {reg }}(\mathrm{L}) \neq \emptyset$}

We have implemented in Maple algorithms to find candidates for $\left[b_{1}, b_{2}\right]$ depending on the cases that we will see after:

1. For ${ }_{2} F_{2}$ we have: findbi2F2ln (logarithmic case) and findbi2F2 (non-logarithmic case);

2. For ${ }_{1} F_{2}$ we have: findbi1F2ln (logarithmic case) and findbi1F2 (non-logarithmic case);

3. For ${ }_{0} F_{2}$ we have: findbiOF2ln (logarithmic case), findbiOF2 (non-logarithmic case);

4. For ${ }_{1} F_{1}^{2}$ we have: findbi1F1sq (non-logarithmic case).

To implement them, we have taken into account the fact that the values of $2 b_{1}, 2 b_{2} \in$ $\mathbb{Z}$ with $b 1 \cdot b 2<0$ or not, and also the fact that $b_{1}$ or $b_{2}$ or $b_{1}-b_{2}$ can be an integer or not if $\mathrm{L}_{0} \in\left\{\mathrm{L}_{22}, \mathrm{~L}_{12}, \mathrm{~L}_{02}\right\}$, and $b_{1}$ can be an integer if $\mathrm{L}_{0}=\mathrm{L}_{11}^{2}$. 


\subsection{1 $\quad \mathrm{S}_{\mathrm{reg}}(\mathrm{L}) \neq \emptyset$}

For $\mathrm{L}_{0} \in\left\{\mathrm{L}_{22}, \mathrm{~L}_{12}, \mathrm{~L}_{02}\right\}$

$\underline{b_{1} \notin \mathbb{Z} \text { and } b_{2} \notin \mathbb{Z} \text { and } b_{1}-b_{2} \notin \mathbb{Z} \text { and }\left(2 b_{1} \text { or } 2 b_{2} \notin \mathbb{Z} \text { or } b 1 \cdot b 2>0\right)}$

We proceed as follows:

1. For $p \in \mathrm{S}_{\mathrm{reg}}(\mathrm{L})$ with $m_{p}$ its multiplicity order as a zero of $f$, the three exponent differences modulo $\mathbb{Z}$ of $\mathrm{L}$ at $p$ are: $\Delta_{1}(\mathrm{~L}, p)=m_{p}\left(1-b_{1}\right), \Delta_{2}(\mathrm{~L}, p)=m_{p}\left(1-b_{2}\right)$ and $\Delta_{3}(\mathrm{~L}, p)=m_{p}\left(b_{1}-b_{2}\right)$. We compute the sets

$$
\begin{aligned}
& \mathrm{N}_{p}^{1}=\left\{\frac{ \pm \Delta_{1}(\mathrm{~L}, p)+j}{m_{p}}|j \in \mathbb{Z},| j \mid \leq m_{p}-1\right\}, \\
& \mathrm{N}_{p}^{2}=\left\{\frac{ \pm \Delta_{2}(\mathrm{~L}, p)+j}{m_{p}}|j \in \mathbb{Z},| j \mid \leq m_{p}-1\right\}, \\
& \mathrm{N}_{p}^{3}=\left\{\frac{ \pm \Delta_{3}(\mathrm{~L}, p)+j}{m_{p}}|j \in \mathbb{Z},| j \mid \leq m_{p}-1\right\} .
\end{aligned}
$$

(a) For $i \in\{1,2,3\}$, we take $\mathrm{N}_{p}^{i}$ as the set of candidates for $b_{1}-b_{2}$. Then the other $\mathrm{N}_{p}^{j}, j \in\{1,2,3\} \backslash\{i\}$ will be the sets of candidates for $1-b_{1}$ and $1-b_{2}$, respectively, since by permuting the values of $b_{1}$ and $b_{2}$ our function $F_{0}$ doesn't change. Hence we can get the sets $\mathrm{N}_{p}^{j 1}$ and $\mathrm{N}_{p}^{j 2}$ of candidates for $b_{1}$ and $b_{2}$. Using Remark 5.6, we replace in $\mathrm{N}_{p}^{i}$ all the rational parts of the elements by their representant modulo $\mathbb{Z}$ in $[-1,1]$, and in $\mathrm{N}_{p}^{j 1}$ and $\mathrm{N}_{p}^{j 2}$ all the rational parts of the elements by their representant modulo $\mathbb{Z}$ in $] 0,1]$. We define

$$
\mathrm{N}_{p}^{j 12}=\left\{\left\{b_{1}, b_{2}\right\} \mid b_{1} \in \mathrm{N}_{p}^{j 1} \text { and } b_{2} \in \mathrm{N}_{p}^{j 2}\right\} .
$$

We remove in $\mathrm{N}_{p}^{j 12}$ all the elements $\left\{b_{1}, b_{2}\right\}$ satisfying

$$
b_{1} \in \mathbb{Z} \text { or } b_{2} \in \mathbb{Z} \text { or } b_{1}-b_{2} \in \mathbb{Z} \text { or }\left(2 b_{1}, 2 b_{2} \in \mathbb{Z} \text { with } b 1 \times b 2<0\right) \text {. }
$$

We take $\mathrm{E}_{p}^{i}=\emptyset$. For any $\left\{b_{1}, b_{2}\right\} \in \mathrm{N}_{p}^{j 12}$, if $b_{1}-b_{2}$ or $b_{2}-b_{1}$ belongs to $\mathrm{N}_{p}^{i}$ then we put $\left\{b_{1}, b_{2}\right\}$ in $\mathrm{E}_{p}^{i}$.

(b) $\mathrm{E}_{p}=\bigcup_{i \in\{1,2,3\}} \mathrm{E}_{p}^{i}$ is the set of candidates for $\left\{b_{1}, b_{2}\right\}$ associated to $p$. If $\mathrm{E}_{p}=\emptyset$ then $b_{1}$ and $b_{2}$ don't exist. That means we cannot solve $\mathrm{L}$ in terms of $F_{0} \in$ $\left\{{ }_{2} F_{2},{ }_{1} F_{2},{ }_{0} F_{2}\right\}$ functions.

2. $\mathrm{E}=\bigcap_{p \in \mathrm{S}_{\mathrm{reg}}(\mathrm{L})} \mathrm{E}_{p}$ is the set of candidates for $\left\{b_{1}, b_{2}\right\}$. If $\mathrm{E}=\emptyset$ then $b_{1}$ and $b_{2}$ don't exist. That means we cannot solve $\mathrm{L}$ in terms of $F_{0} \in\left\{{ }_{2} F_{2},{ }_{1} F_{2},{ }_{0} F_{2}\right\}$ functions.

$b_{1} \in \mathbb{Z}$ or $b_{2} \in \mathbb{Z}$ or $b_{1}-b_{2} \in \mathbb{Z}$ or $\left(2 b_{1}, 2 b_{2} \in \mathbb{Z}\right.$ with $\left.b 1 \cdot b 2<0\right)$

We proceed as in the case $b_{1} \notin \mathbb{Z}$ and $b_{2} \notin \mathbb{Z}$ and $b_{1}-b_{2} \notin \mathbb{Z}$ and $\left(2 b_{1}\right.$ or $2 b_{2} \notin \mathbb{Z}$ or $b 1 \cdot b 2<0$ above. But we replace conditions in (5.2) with

$b_{1} \notin \mathbb{Z}$ and $b_{2} \notin \mathbb{Z}$ and $b_{1}-b_{2} \notin \mathbb{Z}$ and $\left(2 b_{1}\right.$ or $2 b_{2} \notin \mathbb{Z}$ or $\left.b 1 \cdot b 2<0\right)$. 
For $\mathrm{L}_{0}=\mathrm{L}_{11}^{2}$

$\underline{b_{1} \notin \mathbb{Z}}$

We proceed as follows:

1. For $p \in \mathrm{S}_{\mathrm{reg}}(\mathrm{L})$ with $m_{p}$ its multiplicity order as a zero of $f$, the three exponents differences modulo $\mathbb{Z}$ of L at $p$ are: $\Delta_{1}(\mathrm{~L}, p)= \pm m_{p}\left(1-b_{1}\right), \Delta_{2}(\mathrm{~L}, p)= \pm 2 m_{p}\left(1-b_{1}\right)$ and $\Delta_{3}(\mathrm{~L}, p)= \pm m_{p}\left(1-b_{1}\right)$. We choose $i, j, k \in\{1,2,3\}$, all different, satisfying the conditions

$$
\left\{\begin{array}{l}
\Delta_{i}(\mathrm{~L}, p)-\Delta_{j}(\mathrm{~L}, p) \text { or } \Delta_{i}(\mathrm{~L}, p)+\Delta_{j}(\mathrm{~L}, p) \in \mathbb{Z} \\
\Delta_{k}(\mathrm{~L}, p)-2 \cdot \Delta_{i}(\mathrm{~L}, p) \text { or } \Delta_{k}(\mathrm{~L}, p)+2 \cdot \Delta_{i}(\mathrm{~L}, p) \in \mathbb{Z}
\end{array}\right.
$$

Hence $\Delta_{k}(\mathrm{~L}, p)= \pm 2 m_{p}\left(1-b_{1}\right), \Delta_{i}(\mathrm{~L}, p)= \pm m_{p}\left(1-b_{1}\right)$ and $\Delta_{j}(\mathrm{~L}, p)= \pm m_{p}\left(1-b_{1}\right)$. We compute the sets

$$
\begin{aligned}
& \mathrm{N}_{p}^{k}=\left\{\frac{ \pm \Delta_{k}(\mathrm{~L}, p)+j}{2 m_{p}}|j \in \mathbb{Z},| j \mid \leq 2 m_{p}-1\right\}, \\
& \mathrm{N}_{p}^{i}=\left\{\frac{ \pm \Delta_{i}(\mathrm{~L}, p)+j}{m_{p}}|j \in \mathbb{Z},| j \mid \leq m_{p}-1\right\}, \\
& \mathrm{N}_{p}^{j}=\left\{\frac{ \pm \Delta_{j}(\mathrm{~L}, p)+j}{m_{p}}|j \in \mathbb{Z},| j \mid \leq m_{p}-1\right\} .
\end{aligned}
$$

$\mathrm{N}_{p}^{k}, \mathrm{~N}_{p}^{i}$ and $\mathrm{N}_{p}^{j}$ are the sets of candidates for $\pm\left(1-b_{1}\right)$. Hence we can get the sets $\mathrm{N}_{p}^{k 1}, \mathrm{~N}_{p}^{i 1}$ and $\mathrm{N}_{p}^{j 1}$ of candidates for $b_{1}$ using $\mathrm{N}_{p}^{k}, \mathrm{~N}_{p}^{i}$ and $\mathrm{N}_{p}^{j}$, respectively. Using Remark 5.6, we replace in $\mathrm{N}_{p}^{k 1}, \mathrm{~N}_{p}^{i 1}$ and $\mathrm{N}_{p}^{j 1}$ all the rational parts of the elements by their representant modulo $\mathbb{Z}$ in $] 0,1] . \mathrm{E}_{p}=\bigcap_{i \in\{1,2,3\}} \mathrm{N}_{p}^{i 1}$ is the set of candidates for $b_{1}$ associated to $p$. If $\mathrm{E}_{p}=\emptyset$ then $b_{1}$ doesn't exist. That means, we cannot solve $\mathrm{L}$ in terms of $F_{0}={ }_{1} F_{1}^{2}$ functions.

2. $\mathrm{E}=\bigcap_{p \in \mathrm{S}_{\mathrm{reg}}(\mathrm{L})} \mathrm{E}_{p}$ is the set of candidates for $b_{1}$. If $\mathrm{E}=\emptyset$ then $b_{1}$ doesn't exist. That means we cannot solve $\mathrm{L}$ in terms of $F_{0}={ }_{1} F_{1}^{2}$ functions.

\section{$\underline{b_{1} \in \mathbb{Z}}$}

Using Remark 5.6, we can take $b_{1}=1$.

\subsection{2 $\mathrm{S}_{\mathrm{reg}}(\mathrm{L})=\emptyset$}

We will see (in the next sections) that in this case, we first compute the candidates for the change of variable parameter $f$ before computing the candidates for $b_{1}$ and $b_{2}$ for $\mathrm{L}_{0} \in\left\{\mathrm{L}_{22}, \mathrm{~L}_{12}, \mathrm{~L}_{02}\right\}$ and $b_{1}$ for $\mathrm{L}_{0}=\mathrm{L}_{11}^{2}$. Let us assume that we have a candidate for $f$. So we can have all the zeroes and their multiplicity order of $f$. Let $\mathrm{S}$ be the set of all the zeroes of $f$. 
For $\mathrm{L}_{0} \in\left\{\mathrm{L}_{22}, \mathrm{~L}_{12}, \mathrm{~L}_{02}\right\}$

$\underline{b_{1} \notin \mathbb{Z} \text { and } b_{2} \notin \mathbb{Z} \text { and } b_{1}-b_{2} \notin \mathbb{Z} \text { and }\left(2 b_{1} \text { or } 2 b_{2} \notin \mathbb{Z} \text { or } b 1 \cdot b 2>0\right)}$

We proceed as follows:

1. For $p \in \mathrm{S}$ with $m_{p}$ its multiplicity order as a zero of $f$, let us define

$$
\mathrm{N}_{p}=\left\{k \in \mathbb{N}|k| m_{p} \text { and } k \neq 1\right\} \text {. }
$$

$\mathrm{N}_{p}$ is the set of candidates for the denominator of $1-b_{1}, 1-b_{2}$ and $b_{1}-b_{2}$ associated to $p$. Therefore, $\mathrm{N}_{p}$ is the set of candidates for the denominator of $b_{1}, b_{2}$ and $b_{1}-b_{2}$ associated to $p$. We have taken in (5.5) $k \neq 1$ because, if not, $b_{1}$ or $b_{2}$ or $b_{1}-b_{2}$ will be integers and we are not in this case.

2. $\mathrm{N}=\bigcap_{p \in \mathrm{S}} \mathrm{N}_{p}$ is the set of candidates for the denominator of $b_{1}, b_{2}$ and $b_{1}-b_{2}$.

3. Let us define

$$
\left\{\begin{array}{l}
\mathrm{R}_{1}=\left\{\begin{array}{c}
\frac{k}{s} \\
\mathrm{R}_{2}
\end{array}=\left\{\begin{array}{l}
\frac{k}{s}|k \in \mathbb{Z},| k \mid \leq s-1, k \neq 0 \text { and } s \in \mathrm{N} \\
k
\end{array}\right\}, k \leq s-1, k \neq 0 \text { and } s \in \mathrm{N}\right\} .
\end{array}\right.
$$

$\mathrm{R}_{1}$ and $\mathrm{R}_{2}$ are the sets of the representants modulo $\mathbb{Z}$ of candidates for $b_{1}-b_{2}$ in ] $-1,1\left[\right.$ and $b_{1}$ and $b_{2}$ in ]0,1 [, respectively. We have taken in $\mathrm{R}_{1}$ and $\mathrm{R}_{2} k \neq 0$ because, if not, $b_{1}$ or $b_{2}$ or $b_{1}-b_{2}$ will be integers and we are not in this case. We have also considered the candidates for $b_{1}$ and $b_{2}$ in $] 0,1\left[\right.$ and $b_{1}-b_{2}$ in ] $-1,1[$ because of Remark 5.6.

4. $\mathrm{R}=\left\{\{i, j\} \mid i, j \in \mathrm{R}_{2}\right\}$ is the set of candidates for $\left\{b_{1}, b_{2}\right\}$. We remove in $\mathrm{R}$ all the elements $\left\{b_{1}, b_{2}\right\}$ satisfying

$$
b_{1} \in \mathbb{Z} \text { or } b_{2} \in \mathbb{Z} \text { or } b_{1}-b_{2} \in \mathbb{Z} \text { or }\left(2 b_{1}, 2 b_{2} \in \mathbb{Z} \text { with } b 1 \cdot b 2<0\right) \text {. }
$$

5. For any $\left\{b_{1}, b_{2}\right\} \in \mathrm{R}$, if $b_{1}-b_{2}$ or $b_{2}-b_{1}$ belongs to $R_{1}$ then $\left\{b_{1}, b_{2}\right\}$ is a good candidate for the lower parameters of our function. All those good candidates generate a set $\mathrm{E}$. If $\mathrm{E}=\emptyset$ then $b_{1}$ and $b_{2}$ don't exist. That means we cannot solve $\mathrm{L}$ in terms of $F_{0} \in\left\{{ }_{2} F_{2},{ }_{1} F_{2},{ }_{0} F_{2}\right\}$ functions.

$\underline{b_{1} \in \mathbb{Z} \text { or } b_{2} \in \mathbb{Z} \text { or } b_{1}-b_{2} \in \mathbb{Z} \text { or }\left(2 b_{1}, 2 b_{2} \in \mathbb{Z} \text { with } b 1 \cdot b 2<0\right)}$

We proceed as follows:

1. For $p \in \mathrm{S}$ with $m_{p}$ its multiplicity order as a zero of $f$, let us define

$$
\mathrm{N}_{p}=\left\{k \in \mathbb{N}|k| m_{p}\right\} .
$$

$\mathrm{N}_{p}$ is the set of candidates for the denominator of $1-b_{1}, 1-b_{2}$ and $b_{1}-b_{2}$ associated to $p$. Therefore, $\mathrm{N}_{p}$ is the set of candidates for the denominator of $b_{1}, b_{2}$ and $b_{1}-b_{2}$ associated to $p$. We have taken in (5.6) $k=1$ because $b_{1}$ or $b_{2}$ or $b_{1}-b_{2}$ can also be an integer. 
2. $\mathrm{N}=\bigcap_{p \in \mathrm{S}} \mathrm{N}_{p}$ is the set of candidates for the denominator of $b_{1}, b_{2}$ and $b_{1}-b_{2}$.

3. Let us define

$$
\left\{\begin{array}{l}
\mathrm{R}_{1}=\left\{\begin{array}{c}
\frac{k}{s} \\
\mathrm{R}_{2}=\left\{\frac{k}{s}\right. \\
\frac{k}{s}
\end{array} \mid \begin{array}{l}
k \in \mathbb{Z},|k| \leq s, k=0 \text { and } s \in \mathrm{N} \\
k \in s, k=0 \text { and } s \in \mathrm{N}
\end{array}\right\},
\end{array}\right.
$$

$R_{1}$ and $R_{2}$ are the sets of the representants modulo $\mathbb{Z}$ of candidates for $b_{1}-b_{2}$ in $[-1,1]$ and $b_{1}$ and $b_{2}$ in $\left.] 0,1\right]$, respectively. We have taken in $R_{1}$ and $R_{2} k=0$ because $b_{1}$ or $b_{2}$ or $b_{1}-b_{2}$ can also be an integer. We have also considered the candidates for $b_{1}$ and $b_{2}$ in $\left.] 0,1\right]$ and $b_{1}-b_{2}$ in $[-1,1]$ because of Remark 5.6.

4. $\mathrm{R}=\left\{\{i, j\} \mid i, j \in \mathrm{R}_{2}\right\}$ is the set of candidates for $\left\{b_{1}, b_{2}\right\}$. We remove in $\mathrm{R}$ all the elements $\left\{b_{1}, b_{2}\right\}$ satisfying

$$
b_{1} \notin \mathbb{Z} \text { and } b_{2} \notin \mathbb{Z} \text { and } b_{1}-b_{2} \notin \mathbb{Z} \text { and }\left(2 b_{1} \text { or } 2 b_{2} \notin \mathbb{Z} \text { or } b 1 \cdot b 2>0\right) \text {. }
$$

5. For any $\left\{b_{1}, b_{2}\right\} \in \mathrm{R}$, if $b_{1}-b_{2}$ or $b_{2}-b_{1}$ belongs to $R_{1}$ then $\left\{b_{1}, b_{2}\right\}$ is a good candidate for the lower parameters of our function. All those good candidates generate a set $\mathrm{E}$. If $\mathrm{E}=\emptyset$ then $b_{1}$ and $b_{2}$ don't exist. That means we cannot solve $\mathrm{L}$ in terms of $F_{0} \in\left\{{ }_{2} F_{2},{ }_{1} F_{2},{ }_{0} F_{2}\right\}$ functions.

\section{For $\mathrm{L}_{0}=\mathrm{L}_{11}^{2}$}

$\underline{b_{1} \notin \mathbb{Z}}$

We proceed as follows:

1. For $p \in \mathrm{S}$ with $m_{p}$ its multiplicity order as a zero of $f$, let us define

$$
\mathrm{N}_{p}=\left\{k \in \mathbb{N}|k| m_{p} \text { and } k \neq 1\right\} \text {. }
$$

$\mathrm{N}_{p}$ is the set of candidates for the denominator of $\pm\left(1-b_{1}\right)$ associated to $p$. Therefore, $\mathrm{N}_{p}$ is the set of candidates for the denominator of $b_{1}$ associated to $p$. We have taken in (5.7) $k \neq 1$ because, if not, $b_{1}$ will be an integer and we are not in this case.

2. $\mathrm{N}=\bigcap_{p \in \mathrm{S}} \mathrm{N}_{p}$ is the set of candidates for the denominator of $b_{1}$.

3. $\mathrm{E}=\left\{\frac{k}{s} \mid k \in \mathbb{N}, k \leq s-1, k \neq 0\right.$ and $\left.s \in \mathrm{N}\right\}$ is the set of the representants modulo $\mathbb{Z}$ of candidates for $b_{1}$ in ]0,1[. We have taken in $\mathrm{E} k \neq 0$ because, if not, $b_{1}$ will be an integer and we are not in this case. We have also considered the candidates for $b_{1}$ in ]0,1[ because of Remark 5.6. If $\mathrm{E}=\emptyset$ then $b_{1}$ doesn't exist. That means we cannot solve $\mathrm{L}$ in terms of $F_{0}={ }_{1} F_{1}^{2}$ functions.

$\underline{b_{1} \in \mathbb{Z}}$

Using Remark 5.6, we can take $b_{1}=1$. 


\section{$5.4{ }_{2} F_{2}$ and ${ }_{1} F_{1}^{2}$ Type Solutions}

Here the techniques of resolutions are a little bit similar to the Bessel square functions (see the first part of the last chapter). That is due to the form of the exponent differences at 0 and $\infty$ and also to the fact that the ramification index of the generalized exponents at $\infty$ is 1 .

\subsubsection{Parameter $f$ up to a constant}

To find the parameter $f$ up to a constant, we proceed as follows.

1. Let $p \in \mathrm{S}_{\text {irr }}(\mathrm{L})$ with $m_{p}$ its multiplicity order as a pole of $f$.

(a) If $\mathrm{L}_{0}=\mathrm{L}_{22}$, we choose the non-constant exponent difference of $\mathrm{L}$ at $p$. We take its non-constant part: it will be $\sum_{j=-m_{p}}^{-1} j f_{j} t_{p}^{j}$.

(b) If $\mathrm{L}_{0}=\mathrm{L}_{11}^{2}$, we choose the two exponent differences of $\mathrm{L}$ at $p$ which have the same (up to a factor -1 ) non-constant part: this non-constant part will be $\sum_{j=-m_{p}}^{-1} j f_{j} t_{p}^{j}$

By dividing any coefficient of this series by the power of its associated parameter $t_{p}$, we get the polar part of $f$ at $p: \mathrm{F}_{p}= \pm \sum_{j=-m_{p}}^{-1} j f_{j} t_{p}^{j}$.

2. $\mathrm{F}=\sum_{p \in \mathrm{S}_{\mathrm{irr}}(\mathrm{L})} \mathrm{F}_{p}$ is a candidate for $f$ up to a constant.

We have implemented in Maple algorithms called Hyp2F2Subst when $\mathrm{L}_{0}=\mathrm{L}_{22}$ and Hyp1F1sqSubst when $\mathrm{L}_{0}=\mathrm{L}_{11}^{2}$, to find the candidates for $f$ up to a constant.

The problem now is how to find this constant. We will distinguish two cases: when we know at least one zero of $f\left(\mathrm{~S}_{\mathrm{reg}}(\mathrm{L}) \neq \emptyset\right)$ and when we don't know any zero of $f$ $\left(\mathrm{S}_{\mathrm{reg}}(\mathrm{L})=\emptyset\right)$ (integer case).

\subsubsection{No Zero of $f$ is known $\left(\mathrm{S}_{\mathrm{reg}}(\mathrm{L})=\emptyset\right)$}

We call this case the integer case. Here we have absolutely no information about the zeroes of $f$ and all the exponent differences of $\mathrm{L}$ at $p \notin \mathrm{S}_{\mathrm{irr}}(\mathrm{L})$ are integers. What we know is just the candidates for $f$ modulo a constant $c \in k$.

Definition 5.8. For $m \in \mathbb{N}$, we can define

$$
\mathrm{N}(m)=\left\{\frac{j}{m}|j \in \mathbb{Z}, j \neq 0| j \mid \leq m-1\right\} .
$$

Remark 5.9. By using Definition 5.8 and taking, in the Bessel square type solutions case (see the first part of the last chapter), instead of $2 \nu$ respectively $1-b_{2}$ and $b_{1}-b_{2}$ for $\mathrm{L}_{0}=\mathrm{L}_{22}$ and $1-b_{1}$ for $\mathrm{L}_{0}=\mathrm{L}_{11}^{2}$, Lemma 4.4, Lemma 4.5 and Corollary 4.6 hold also here (in this case). Let $n$ be the degree of the numerator of $f$ and for $i \in\{1,2\}$, $m_{i}=$ denom $\left(b_{i}\right)$. Then $m_{i}$ divides $n$ and the lower parameter $b_{i}$ appears modulo an integer in $\mathrm{N}\left(m_{i}\right)(i \in\{1,2\})$. 
In Definition 5.8, $0 \notin \mathrm{N}(m)$ because, if not, the lower parameter can be an integer and we are not in this case: the logarithmic case with $\mathrm{S}_{\mathrm{reg}}(\mathrm{L}) \neq \emptyset$ (we will see this later).

Since we know how to compute the lower parameter(s) of $F_{0} \in\left\{{ }_{2} F_{2},{ }_{1} F_{1}^{2}\right\}$, we can see, using the exponent differences of $\mathrm{L}$ at elements of $\mathrm{S}_{\text {irr }}(\mathrm{L})$, how to get upper parameter(s).

\section{How to compute $a_{1}$ and $a_{2}$}

Let us assume that we know the lower parameter(s) of $F_{0} \in\left\{{ }_{2} F_{2},{ }_{1} F_{1}^{2}\right\}$. To have candidates for the upper parameter(s), we proceed as follows:

1. We take a candidate for the lower parameter(s) of $F_{0} \in\left\{{ }_{2} F_{2},{ }_{1} F_{1}^{2}\right\}:\left\{b_{1}, b_{2}\right\}$ if $F_{0}={ }_{2} F_{2}$ and $b_{1}$ if $F_{0}={ }_{1} F_{1}^{2}$.

2. Let $p \in \mathrm{S}_{\text {irr }}(\mathrm{L})$ with $m_{p}$ its multiplicity order as a pole of $f$.

(a) If $\mathrm{L}_{0}=\mathrm{L}_{22}$,

i. We call $\mathrm{C}_{p}^{1}$ the constant exponent difference of $\mathrm{L}$ at $p$ : $\mathrm{C}_{p}^{1}=m_{p}\left(a_{2}-\right.$ $a_{1}$ ) modulo $\mathbb{Z}$. We call $\mathrm{C}_{p}^{2}$ and $\mathrm{C}_{p}^{3}$ the constant part of the other exponent differences: $\mathrm{C}_{p}^{2}=m_{p}\left[b_{1}+b_{2}-\left(2 a_{1}+a_{2}\right)\right]$ modulo $\mathbb{Z}$ and $\mathrm{C}_{p}^{3}=$ $m_{p}\left[b_{1}+b_{2}-\left(a_{1}+2 a_{2}\right)\right]$ modulo $\mathbb{Z}$.

ii. We compute the sets $\mathrm{N}_{p}^{1}, \mathrm{~N}_{p}^{2}$ and $\mathrm{N}_{p}^{3}$ of candidates modulo $\mathbb{Z}$ for $\pm\left(a_{2}-a_{1}\right)$, $\pm\left(2 a_{1}+a_{2}\right)$ and $\pm\left(a_{1}+2 a_{2}\right)$, respectively:

$$
\left\{\begin{array}{l}
\mathrm{N}_{p}^{1}=\left\{\frac{j \pm \mathrm{C}_{p}^{1}}{m_{p}}|j \in \mathbb{Z},| j \mid \leq m_{p}-1\right\}, \\
\mathrm{N}_{p}^{2}=\left\{b_{1}+b_{2}-\frac{j \pm \mathrm{C}_{p}^{2}}{m_{p}}|j \in \mathbb{Z},| j \mid \leq m_{p}-1\right\}, \\
\mathrm{N}_{p}^{3}=\left\{b_{1}+b_{2}-\frac{j \pm \mathrm{C}_{p}^{3}}{m_{p}}|j \in \mathbb{Z},| j \mid \leq m_{p}-1\right\} .
\end{array}\right.
$$

iii. We compute $\mathrm{N}_{p}^{4}=\left\{\frac{i+j+s}{3} \mid i \in \mathrm{N}_{p}^{2}\right.$ and $\left.j \in \mathrm{N}_{p}^{3},|s| \leq 2\right\}$. $\mathrm{N}_{p}^{4}$ is the set of candidates modulo $\mathbb{Z}$ for $\pm\left(a_{1}+a_{2}\right)$. Hence,

$$
\mathrm{N}_{p}^{5}=\left\{\frac{i+j+s}{2} \mid i \in \mathrm{N}_{p}^{4} \text { and } j \in \mathrm{N}_{p}^{1},|s| \leq 1\right\}
$$

is the set of candidates modulo $\mathbb{Z}$ for $\pm a_{2}$ and

$$
\mathrm{N}_{p}=\left\{(i-j, i) \mid i \in \mathrm{N}_{p}^{5} \text { and } j \in \mathrm{N}_{p}^{1}\right\}
$$

is the set of candidates modulo $\mathbb{Z} \times \mathbb{Z}$ for $\left(a_{1}, a_{2}\right)$. We replace in $\mathrm{N}_{p}$ all the rational parts of the elements in the pair by their representants modulo $\mathbb{Z}$ in $[0,1]$.

(b) If $\mathrm{L}_{0}=\mathrm{L}_{11}^{2}$,

i. We choose the two exponent differences of $\mathrm{L}$ at $p$ which have the same (up to a factor -1) non-constant part. The constant part of those exponent differences are

$$
\mathrm{C}_{p}^{1}= \pm m_{p}\left(b_{1}-2 a_{1}\right) \text { modulo } \mathbb{Z} \text { and } \mathrm{C}_{p}^{2}= \pm m_{p}\left(b_{1}-2 a_{1}\right) \text { modulo } \mathbb{Z} \text {. }
$$


ii. We compute the set $\mathrm{N}_{p}$

$$
\mathrm{N}_{p}=\left\{\left(b_{1}-\frac{j \pm \mathrm{C}_{p}^{1}}{m_{p}}\right) \cdot \frac{1}{2}|j \in \mathbb{Z},| j \mid \leq 2 m_{p}-1\right\}
$$

iii. We replace in $\mathrm{N}_{p}$ all the rational part of the element by their representant modulo $\mathbb{Z}$ in $[0,1]$.

3. $\mathrm{E}=\bigcap_{p \in \mathrm{S}_{\text {irr }}(\mathrm{L})} \mathrm{N}_{p}$ is the set of candidates for $\left(a_{1}, a_{2}\right)$ if $\mathrm{L}_{0}=\mathrm{L}_{22}$, and $a_{1}$ if $\mathrm{L}_{0}=\mathrm{L}_{11}^{2}$. If $\mathrm{E}=\emptyset$, the value

(a) of $b_{1}$ or $b_{2}$ when $\mathrm{L}_{0}=\mathrm{L}_{22}$ does not lead to a solution,

(b) of $b_{1}$ when $\mathrm{L}_{0}=\mathrm{L}_{11}^{2}$ does not lead to a solution.

So we take another candidate for the lower parameter(s) of $F_{0} \in\left\{{ }_{2} F_{2},{ }_{1} F_{1}^{2}\right\}$ and we repeat all the process.

We have implemented in Maple algorithms called findcandai2F2 and findcandai1F1sq to find candidates for the upper parameter(s) of $F_{0}={ }_{2} F_{2}$ and $F_{0}={ }_{1} F_{1}^{2}$, respectively, if they exist.

\section{Conclusion}

To find candidates for $\left[\left\{a_{1}, a_{2}\right\},\left\{b_{1}, b_{2}\right\}, f\right]$ when $F_{0}={ }_{2} F_{2}$ and $\left[\left\{a_{1}\right\}, b_{1}, f\right]$ when $F_{0}={ }_{1} F_{1}^{2}$ we proceed as follows:

1. We compute the polar part of $f$ : that can be done by our algorithm Hyp2F2Subst if $\mathrm{L}_{0}=\mathrm{L}_{22}$ and Hyp1F1sqSubst if $\mathrm{L}_{0}=\mathrm{L}_{11}^{2}$. Hence, we get candidates for $f$ modulo an additive constant.

2. For $g$ candidate for the polar part of $f$, we have to find, if it exists, $c \in k$ such that $f=g+c$.

(a) We take $n$ as in Corollary 4.6

$$
n= \begin{cases}\operatorname{deg}(\operatorname{numer}(g+c)) & \text { if } \infty \in \mathrm{S}_{\mathrm{irr}}(\mathrm{L}), \\ \operatorname{deg}(\operatorname{denom}(g+c)) & \text { otherwise }\end{cases}
$$

(b) $\operatorname{denom}\left(1-b_{1}\right)$, denom $\left(1-b_{2}\right)$, and denom $\left(b_{1}-b_{2}\right)$ must divide $n$ (by Remark 5.9 and the proof of Lemma 4.4 ): if $n=1$ then $b_{1}, b_{2} \in \mathbb{Z}$ for $\mathrm{L}_{0}=\mathrm{L}_{22}$ or $b_{1} \in \mathbb{Z}$ for $\mathrm{L}_{0}=\mathrm{L}_{11}^{2}$ (logarithmic case: we will consider this case afterwards). So $n>1$ and denom $\left(b_{1}\right)$, denom $\left(b_{2}\right) \geq 2$ for $\mathrm{L}_{0}=\mathrm{L}_{22}$ or $\operatorname{denom}\left(b_{1}\right) \geq 2$ for $\mathrm{L}_{0}=\mathrm{L}_{11}^{2}$. For $n \leq 1$, we will say that $g+c, \forall c \in k$, is not a good candidate for $f$ and then we will return to step 2. and take another candidate $g$ for the polar part of $f$.

(c) We take all the divisors $m$ of $n$ greater or equal to 2 . Let $\mathcal{C}$ be the set of those divisors. Hence $\mathcal{C}$ is the set of candidates for the denominators of $b_{1}$ and $b_{2}$ when $\mathrm{L}_{0}=\mathrm{L}_{22}$ or the denominator of $b_{1}$ when $\mathrm{L}_{0}=\mathrm{L}_{11}^{2}$. 
i. If $\mathrm{L}_{0}=\mathrm{L}_{22}$, let $\left(m_{1}, m_{2}\right) \in \mathcal{C} \times \mathcal{C}$. We check whether for certain constants $c \in$ $k$ the monic part of the numerator of $g+c$ becomes at the same time an $m_{1}^{t h}$ and an $m_{2}^{\text {th }}$ power. This can simply be done using linear algebra and leads to a linear system of equations for the constant $c$. Solving these equations gives us a set $\mathcal{C}_{\left(m_{1}, m_{2}\right)}$ of possible values for $c$ related to $\left(m_{1}, m_{2}\right)$. If $\mathcal{C}_{\left(m_{1}, m_{2}\right)}=\emptyset,\left(m_{1}, m_{2}\right)$ is not a good candidate for $\left(\operatorname{denom}\left(b_{1}\right), \operatorname{denom}\left(b_{2}\right)\right)$ and then we will return and take another element $\left(m_{1}, m_{2}\right)$ in $\mathcal{C} \times \mathcal{C}$.

- $\forall c \in \mathcal{C}_{\left(m_{1}, m_{2}\right)}$, we compute $g+c$. These will give us the set of candidates $\mathrm{F}_{\left(m_{1}, m_{2}\right)}$ for $f$ related to $\left(m_{1}, m_{2}\right)$. Let us compute also $\mathrm{N}\left(m_{1}\right)$ and $\mathrm{N}\left(m_{2}\right)$.

- For $\bar{g} \in \mathrm{F}_{\left(m_{1}, m_{2}\right)}$,

* For $\left(b_{1}, b_{2}\right) \in \mathrm{N}\left(m_{1}\right) \times \mathrm{N}\left(m_{2}\right)$, we compute the set B of candidates for $\left\{a_{1}, a_{2}\right\}$ (we have shown that above). If $\mathrm{B} \neq \emptyset$ then $\left[\mathrm{B},\left\{b_{1}, b_{2}\right\}, \bar{g}\right]$ is a good candidate for $\left[\mathrm{B}_{1},\left\{b_{1}, b_{2}\right\}, f\right]$ where $\mathrm{B}_{1}$ is the set of candidates for $\left\{a_{1}, a_{2}\right\}$. All those candidates generate a set $\mathrm{G}_{\left(m_{1}, m_{2}\right)}$.

* If a set $\mathrm{G}_{\left(m_{1}, m_{2}\right)} \neq \emptyset$ is not generated then $\bar{g}$ is not a good candidate for $f$. Then we will return and take another $\bar{g}$ in $\mathrm{F}_{\left(m_{1}, m_{2}\right)}$.

We define a set $\mathrm{G}_{g}$ to be the union of the sets $\mathrm{G}_{\left(m_{1}, m_{2}\right)}$, with $\left(m_{1}, m_{2}\right) \in$ $\mathcal{C} \times \mathcal{C}$.

ii. If $\mathrm{L}_{0}=\mathrm{L}_{11}^{2}$, let $m_{1} \in \mathcal{C}$. We check whether for certain constants $c \in k$ the monic part of the numerator of $g+c$ becomes an $m_{1}^{\text {th }}$ power. This can simply be done with linear algebra and leads to a linear system of equations for the constant $c$. Solving these equations gives us a set $\mathcal{C}_{m_{1}}$ of possible values for $c$ related to $m_{1}$. If $\mathcal{C}_{m_{1}}=\emptyset, m_{1}$ is not a good candidate for the denominator of $b_{1}$ and then we will return and take another element $m_{1}$ in $\mathcal{C}$.

- $\forall c \in \mathcal{C}_{m_{1}}$, we compute $g+c$. These will give us the list of candidates $\mathrm{F}_{m_{1}}$ for $f$ related to $m_{1}$. Let us compute also $\mathrm{N}\left(m_{1}\right)$.

- For $\bar{g} \in \mathrm{F}_{m_{1}}$

* For $b_{1} \in \mathrm{N}\left(m_{1}\right)$, we compute the set B of candidates for $a_{1}$ (we have shown that above). If $\mathrm{B} \neq \emptyset$ then $\left[\mathrm{B}, b_{1}, \bar{g}\right]$ is a good candidate for $\left[\mathrm{B}_{1}, b_{1}, f\right]$ where $\mathrm{B}_{1}$ is the set of candidates for $a_{1}$. All those candidates generate a set $\mathrm{G}_{m_{1}}$.

* If a set $\mathrm{G}_{m_{1}} \neq \emptyset$ is not generated then $\bar{g}$ is not a good candidate for $f$. Then we will return and take another $\bar{g}$ in $\mathrm{F}_{m_{1}}$.

We define a set $\mathrm{G}_{g}$ to be the union of the sets $\mathrm{G}_{m_{1}}$, with $m_{1} \in \mathcal{C}$.

(d) If $\mathrm{G}_{g} \neq \emptyset$ then $\mathrm{G}_{g}$ is a good set in which we can have candidates for $\left[\left\{a_{1}, a_{2}\right\},\left\{b_{1}\right.\right.$, $\left.\left.b_{2}\right\}, f\right]$ when $F_{0}={ }_{2} F_{2}$ and $\left[\left\{a_{1}\right\}, b_{1}, f\right]$ when $F_{0}={ }_{1} F_{1}^{2}$. If not, $g$ is not a good candidate for the polar part of $f$. Then we will return and take another candidate $g$ for the polar part of $f$.

3. We define a set $\mathrm{A}$ to be the union of the sets $\mathrm{G}_{g}$, with $g$ a candidate for the polar part of $f$. If $\mathrm{A} \neq \emptyset$, then $\mathrm{L}$ has $F_{0}$ type solutions, if not, we cannot solve $\mathrm{L}$ in terms of $F_{0}$ functions.

We have implemented in Maple an algorithm called find2F2Int to find candidates for $\left[\left\{a_{1}, a_{2}\right\},\left\{b_{1}, b_{2}\right\}, f\right]$ when $F_{0}={ }_{2} F_{2}$, and another called $\mathrm{f}$ ind1F1sqInt to find candidates 
for $\left[\left\{a_{1}\right\}, b_{1}, f\right]$ when $F_{0}={ }_{1} F_{1}^{2}$, if they exist.

\subsubsection{Some Zeroes of $f$ are known $\left(\mathrm{S}_{\mathrm{reg}}(\mathrm{L}) \neq \emptyset\right)$}

The technique here is similar to the Bessel square case with (see the first part of the last chapter). Instead of Lemma 4.8 we take Lemma 5.7. Remark 4.9 also holds.

We will distinguish two cases:

- the logarithmic case: the lower parameter(s) of $F_{0}$ satisfy

$* b_{1} \in \mathbb{Z}$ or $b_{2} \in \mathbb{Z}$ or $b_{1}-b_{2} \in \mathbb{Z}$ or $\left(2 b_{1}, 2 b_{2} \in \mathbb{Z}\right.$ with $\left.b 1 \cdot b 2<0\right)$ for $F_{0}={ }_{2} F_{2}$, or

$* b_{1} \in \mathbb{Z}$ for $F_{0}={ }_{1} F_{1}^{2}$

- the non-logarithmic case: we are not in the logarithmic case.

\section{Logarithmic Case}

By Remark 4.9 (which holds here), $\mathrm{S}_{\text {reg }}(\mathrm{L})$ represents all the zeroes of $f$. So $\mathrm{S}_{\text {reg }}(\mathrm{L}) \neq \emptyset$ in this case.

To find candidates for $\left(\left\{a_{1}, a_{2}\right\},\left\{b_{1}, b_{2}\right\}, f\right)$ we proceed as follows:

1. We compute the polar part of $f$ : that can be done by our algorithm Hyp2F2Subst when $\mathrm{L}_{0}=\mathrm{L}_{22}$ and Hyp1F1sqSubst when $\mathrm{L}_{0}=\mathrm{L}_{11}^{2}$. Hence, we get a set of candidates for $f$ modulo an additive constant. Let us denote this set by $\mathrm{F}$.

2. For $g \in \mathrm{F}$ (candidate for the polar part of $f$ )

(a) We use an element of $\mathrm{S}_{\text {reg }}(\mathrm{L})$ to find this constant $c$. Let $\bar{g}=g+c$.

(b) We test if the other elements in $\mathrm{S}_{\text {reg }}(\mathrm{L})$ are also zeroes of $\bar{g}$. If that is true then $g$ is a good candidate for the polar part of $f$. If not, we return and take another $g$ in $\mathrm{F}$.

We have implemented in Maple algorithms called Candichangvar2F2 when $\mathrm{L}_{0}=\mathrm{L}_{22}$ and Candichangvar1F1sq when $\mathrm{L}_{0}=\mathrm{L}_{11}^{2}$, to find $\bar{g}=g+c$. All those elements $g$ for which this is true generate a set $\overline{\mathrm{F}}$.

3. If $\overline{\mathrm{F}}=\emptyset$, then $\mathrm{L}$ has not $F_{0} \in\left\{{ }_{2} F_{2},{ }_{1} F_{1}^{2}\right\}$ type solutions.

4. For $\bar{g} \in \overline{\mathrm{F}}$

(a) we compute the set of candidates for the lower parameter(s) of $F_{0}$ (we have shown above how to get this set). Let $\mathrm{E}$ be this set. If $\mathrm{E}=\emptyset, \bar{g} \in \overline{\mathrm{F}}$ is not a good candidate for $f$ and then we take another $\bar{g}$ in $\overline{\mathrm{F}}$.

(b) For an element $b$ of E, we compute the set for candidates for the upper parameter(s) of $F_{0}$ (we have shown above how to get this set). Let $\mathrm{B}_{b}$ be this set. If $\mathrm{B}_{b}=\emptyset$, the chosen candidate $b$ for the lower parameter(s) is not good and then we take another candidate $b$ in $\mathrm{E}$.

All the lists $\left[\mathrm{B}_{b}, b, \bar{g}\right]$ for which $\mathrm{B}_{b} \neq \emptyset$ generate a set $\mathrm{A}$. 
5. If $\mathrm{A} \neq \emptyset$ then $\mathrm{L}$ has $F_{0}$ type solutions, if not, we cannot solve $\mathrm{L}$ in terms of $F_{0} \in\left\{{ }_{2} F_{2},{ }_{1} F_{1}^{2}\right\}$ functions.

We have implemented in Maple algorithms called find2F2ln when $\mathrm{L}_{0}=\mathrm{L}_{22}$ and $\mathrm{find}$ $1 \mathrm{~F} 1 \mathrm{sqln}$ when $\mathrm{L}_{0}=\mathrm{L}_{11}^{2}$, to find at the same time candidates for $f$, lower and upper parameter(s) of $F_{0} \in\left\{{ }_{2} F_{2},{ }_{1} F_{1}^{2}\right\}$, if they exist.

\section{Non-Logarithmic Case}

In this case we know at least one zero of $f\left(S_{\text {reg }}(L) \neq \emptyset\right)$, and we have the following conditions on the lower parameter(s) of $F_{0}$ :

1. $b_{1} \notin \mathbb{Z}$ and $b_{2} \notin \mathbb{Z}$ and $b_{1}-b_{2} \notin \mathbb{Z}$ and $\left(2 b_{1}\right.$ or $2 b_{2} \notin \mathbb{Z}$ or $\left.b 1 \cdot b 2<0\right)$ when $\mathrm{L}_{0}=\mathrm{L}_{11}^{2}$,

2. $b_{1} \notin \mathbb{Z}$ when $\mathrm{L}_{0}=\mathrm{L}_{11}^{2}$.

To find candidates for $\left(\left\{a_{1}, a_{2}\right\},\left[b_{1}, b_{2}\right], f\right)$ we proceed as in the logarithmic case, but with some considerations in the computations:

1. for $\mathrm{L}$, some elements of $\mathrm{S}_{\mathrm{reg}}(\mathrm{L})$ can have exponent differences not in $\mathbb{Q}$ or $k$,

2. the constant parts of the exponent differences of $L$ at some elements of $S_{\text {irr }}(L)$ can be not in $\mathbb{Q}$ or $k$.

So, we need sometimes to work in an extension field of $\mathbb{Q}$, and in some cases in an extension field of $k(\mathbb{Q} \subset k)$. Hence, we will take into consideration all the extension fields of $\mathbb{Q}$ or $k$ coming from the exponent differences of $\mathrm{L}$ at every element of $\mathrm{S}_{\text {reg }}(\mathrm{L})$ and $\mathrm{S}_{\text {irr }}(\mathrm{L})$. For $\mathrm{S}_{\text {irr }}(\mathrm{L})$, we need just to consider the extension field of $\mathbb{Q}$ or $k$ coming from the constant part of the exponent differences of $\mathrm{L}$ at all its elements. This case combines similar cases as the rational, basefield and irrational cases from the Bessel square type solutions (see the first part of the last chapter).

For this case, we have implemented in Maple algorithms called find2F2RatIrr when $\mathrm{L}_{0}=\mathrm{L}_{22}$ and find1F1sqRatIrr when $\mathrm{L}_{0}=\mathrm{L}_{11}^{2}$, to find at same time candidates for $f$, lower and upper parameter(s) of $F_{0} \in\left\{{ }_{2} F_{2},{ }_{1} F_{1}^{2}\right\}$ if they exist.

\subsubsection{Examples}

We have written some algorithms on how to find $F_{0} \in\left\{{ }_{2} F_{2},{ }_{1} F_{1}^{2}\right\}$ type solutions if they exist. We have also summarized them by writing another algorithms called Hyp2F2Solutions when $\mathrm{L}_{0}=\mathrm{L}_{22}$ and Hyp1F1sqSolutions when $\mathrm{L}_{0}=\mathrm{L}_{11}^{2}$, which take as input a third-order linear differential operator $\mathrm{L}$ and return, if they exist, all the parameters of transformations $\left(r, r_{2}, r_{1}, r_{0}, f \in k(x)\right)$ and also the upper and lower parameter(s) of $F_{0} \in\left\{{ }_{2} F_{2},{ }_{1} F_{1}^{2}\right\}$ such that we are in situation (5.1)

$$
\mathrm{L}_{0} \stackrel{f}{\longrightarrow} C \mathrm{M} \longrightarrow E G \mathrm{~L}
$$

with $\mathrm{L}_{0} \in\left\{\mathrm{L}_{22}, \mathrm{~L}_{11}^{2}\right\}$. If not, it will return "No $F_{0}$ type solutions". Those algorithms deal with all the cases and give us the short solutions, if they exist, associated to the upper and lower parameter(s) of $F_{0}$.

We will take here just one example, depending on $F_{0} \in\left\{{ }_{2} F_{2},{ }_{1} F_{1}^{2}\right\}$, and show explicitly how some of our algorithms work. 


\section{${ }_{2} F_{2}$ type solutions}

Let us consider the differential operator $\mathrm{L}_{22}$ associated to the ${ }_{2} F_{2}$ hypergeometric function with parameters (upper and lower) $a_{1}=1 / 7, a_{2}=1 / 3, b_{1}=1$ and $b_{2}=1 / 12$. To get $\mathrm{L}_{22}$ with Maple we proceed as follows:

$>$ eq:=sumdiffeq(hyperterm([a1,a2], [b1, b2], x, i), i, J(x));

$$
\begin{aligned}
e q:= & \left(\frac{d^{3}}{d x^{3}} J(x)\right) x^{2}+(-x+b 2+1+b 1)\left(\frac{d^{2}}{d x^{2}} J(x)\right) x \\
& -(x a 1+x a 2-b 2 b 1+x) \frac{d}{d x} J(x)-a 1 a 2 J(x)=0
\end{aligned}
$$

$>$ LA: $=\operatorname{de} 2 \operatorname{diffop}($ eq, $\mathrm{J}(\mathrm{x}))$;

$$
\begin{gathered}
L A:=x^{2} D x^{3}+\left(-x^{2}+x b 2+x+x b 1\right) D x^{2}+(-x a 1-x a 2+b 2 b 1-x) D x-a 1 a 2 \\
>\quad \text { L22: }=\operatorname{subs}(\{\mathrm{a} 1=1 / 7, \mathrm{a} 2=1 / 3, \mathrm{~b} 1=1, \mathrm{~b} 2=1 / 12\}, \mathrm{LA}) ; \\
L 22:=x^{2} D x^{3}+\left(-x^{2}+\frac{25}{12} x\right) D x^{2}+\left(-\frac{31}{21} x+1 / 12\right) D x-\frac{1}{21}
\end{gathered}
$$

Let us apply to $\mathrm{L}_{22}$ the change of variables transformation with parameter $f$ given by

$$
\begin{aligned}
>f:=(2 *(x-7) \wedge 4 *(x-12)) & /((x-3) \sim 2) ; \\
f & :=2 \frac{(x-7)^{4}(x-12)}{(x-3)^{2}}
\end{aligned}
$$

That gives us the following operator called L:

$$
\begin{aligned}
>\mathrm{L}:= & \text { ChangeOfVariables }(\mathrm{L} 22, \mathrm{f}) ; \\
L:= & 84(x-12)^{2}(x-7)^{3}\left(3 x^{2}-32 x-3\right)^{3}(x-3)^{5} D x^{3}-7(x-12)\left(216 x^{9}\right. \\
& -13248 x^{8}-4922601 x^{6}+41680902 x^{5}-208564471 x^{4}+565736148 x^{3} \\
& \left.+344544 x^{7}-609105879 x^{2}-131038290 x-3472713\right)(x-3)^{2}(x-7)^{2} \\
& \times\left(3 x^{2}-32 x-3\right)^{2} D x^{2}-(x-3)(x-7)\left(3 x^{2}-32 x-3\right)\left(-910656 x^{12}\right. \\
& -692939925 x^{10}+9119049254 x^{9}-76891672763 x^{8}+16184128819722 x^{4} \\
& +33121152 x^{11}+394635286912 x^{7}-944552894530 x^{6}-1376352434380 x^{5} \\
& +11016 x^{13}-41634936222768 x^{3}+34489504598535 x^{2}+7779400339662 x \\
& +210612181185) D x-8(x-7)^{4}\left(3 x^{2}-32 x-3\right)^{6}
\end{aligned}
$$

Let us assume that we have as input this operator L and then see how with our codes we can find its ${ }_{2} F_{2}$ type solutions if they exist. That means if we can find the hypergeometric parameters $a_{1}, a_{2}, b_{1}$ and $b_{2}$ of ${ }_{2} F_{2}$ and the transformation parameters such that

$$
\mathrm{L}_{22} \stackrel{f}{\longrightarrow}_{C} \mathrm{M} \longrightarrow{ }_{E G} \mathrm{~L} .
$$

Let ext be the set of all non-rational numbers, parameters and names (except the variable $x$ ) which appear in the expression of the coefficients of $\mathrm{L}$ :

$$
\begin{gathered}
>\operatorname{ext}:=\operatorname{indets}(\mathrm{L},\{\operatorname{Root} 0 \mathrm{f}, \mathrm{name}\}) \operatorname{minus}\{\mathrm{x}, \mathrm{Dx}\} ; \\
\text { ext }:=\{\}
\end{gathered}
$$

Let extp be the set of all algebraic numbers given by the RootOf expressions in ext such that all its elements are algebraic over the minimal field containing $\mathbb{Q} \cup$ (ext $\backslash$ extp). 
Since sometimes we can use in our computations the RootOf expression associated with its index, we will take extp $=\{\}$.

Let us denote by E the set of all the singularities of L (we can find E using our implemented code called Singular).

$>\mathrm{E}:=$ Singular $(\mathrm{L}, \mathrm{extp})$ :

Then the set of non-apparent singularities of $\mathrm{L}$ is given by

$>\mathrm{F}:=\operatorname{NotAppSing}(\mathrm{L}, \mathrm{E}$, ext $)$;

$$
F:=[[\infty, \infty],[x-3,3],[x-7,7],[x-12,12]]
$$

Let $S_{\text {irr }}$ be the set of irregular singularities of $\mathrm{L}$ which is the set of poles of $f$.

$>\operatorname{Sirr}:=\operatorname{irrsing} 2 \mathrm{~F} 2(\mathrm{~L}, \mathrm{t}, \mathrm{F}, \mathrm{ext})$;

$$
\begin{aligned}
& \operatorname{Sirr}:=\left[[[\infty, \infty],[x-3,3]],\left[\left[1, \frac{3}{7},-6 t^{-3}+136 t^{-2}-834 t^{-1}+\frac{51}{28}\right],\left[\frac{2}{3}, \frac{2}{7},\right.\right.\right. \\
&\left.\left.9216 t^{-2}-5120 t^{-1}+\frac{17}{14}\right]\right],\left[\left[-6 t^{-3}+136 t^{-2}-834 t^{-1}+\frac{23}{28},-\frac{4}{7}\right],\right. \\
& {\left.\left.\left[9216 t^{-2}-5120 t^{-1}+\frac{23}{42},-\frac{8}{21}\right]\right],[3,2]\right] }
\end{aligned}
$$

Let us take $\mathrm{G}$ as the set of elements in $\mathrm{F}$ at which $\mathrm{L}$ has all its exponent differences in $k$. Let $\mathrm{S}_{\text {reg }}$ be the set of elements in $\mathrm{G}$ at which $\mathrm{L}$ has logarithmic solutions or all its exponent differences are not, at same time, in $\mathbb{N}$. All the elements of $S_{\text {reg }}$ belong to the set of all the zeroes of $f$ and they are given by

$$
>\text { Sreg:=regsingtrue2F2 (L,t, } \operatorname{Sirr}[-1] \text {, ext) ; }
$$

$$
\text { Sreg }:=\left[[[x-7,7],[x-12,12]],\left[\left[0,0, \frac{11}{3}\right],\left[0,0, \frac{11}{12}\right]\right],\left[\left[0, \frac{11}{3}, \frac{11}{3}\right],\left[0, \frac{11}{12}, \frac{11}{12}\right]\right]\right]
$$

$\mathrm{S}_{\text {reg }}$ can be divided into two subsets: the set of non-removable singularities of L (at least one exponent difference is not an integer or $\mathrm{L}$ has logarithmic solution(s)) and a set of removable singularities of $\mathrm{L}$ (all the exponent differences are integers and $\mathrm{L}$ doesn't have logarithmic solution(s)). The set of non-removable singularities of L can also be divided into two sets: the singularities at which $\mathrm{L}$ has logarithmic solutions and the singularities at which $\mathrm{L}$ doesn't have logarithmic solutions. Hence, let us denote by $\mathrm{RS}_{\text {reg }}$ a set of three sets: the first is the set of non-removable singularities of $\mathrm{L}$ with non-logarithmic solutions, the second is the set of removable singularities of $\mathrm{L}$ in $\mathrm{S}_{\mathrm{reg}}$, and the third is the set of singularities of $\mathrm{L}$ with logarithmic solutions. With our code called Sregseptrue2F2 we can find this set $\mathrm{RS}_{\mathrm{reg}}$ :

> RSreg:= Sregseptrue2F2(L, Sreg, ext);

$$
\begin{aligned}
\text { RSreg }:=\left[[],[],\left[[[x-7,7],[x-12,12]],\left[\left[0,0, \frac{11}{3}\right],\left[0,0, \frac{11}{12}\right]\right],\right.\right. \\
{\left.\left.\left[\left[\left[\frac{11}{3}, \frac{11}{3}\right],[0]\right],\left[\left[\frac{11}{12}, \frac{11}{12}\right],[0]\right]\right]\right]\right] }
\end{aligned}
$$

Since the third element in $\mathrm{RS}_{\text {reg }}$ is not an empty list, there exists a singularity of $\mathrm{L}$ with logarithmic solutions. That means we are in the logarithmic case and we know all the zeroes of $f$.

In order to make our codes faster, we have implemented one procedure called IrrRegAppsing2F2 which gives at the same time and, by avoiding duplicate computations, in a shorter time all the outputs of the procedures irrsing2F2, regsingtrue2F2 
and Sregseptrue2F2.

$>\mathrm{R} 1:=\operatorname{Irr} R e g A p p \operatorname{sing} 2 \mathrm{~F} 2(\mathrm{~L}, \mathrm{t}, \mathrm{E}, \mathrm{ext})$ :

With our code called Hyp2F2Subst, we can get the candidates for $f$ up to an additive constant (the polar part of $f$ ).

$>\mathrm{F} 1:=\operatorname{Hyp} 2 \mathrm{~F} 2 \operatorname{Subst}(\mathrm{L}, \mathrm{x}, \mathrm{t}, \mathrm{R} 1[1], \mathrm{ext})$;

$$
\begin{gathered}
F 1:=\left[-2 x^{3}+68 x^{2}-834 x-4608(x-3)^{-2}+5120(x-3)^{-1},-2 x^{3}+68 x^{2}-834 x\right. \\
+4608(x-3)^{-2}-5120(x-3)^{-1}, 2 x^{3}-68 x^{2}+834 x-4608(x-3)^{-2} \\
\left.+5120(x-3)^{-1}, 2 x^{3}-68 x^{2}+834 x+4608(x-3)^{-2}-5120(x-3)^{-1}\right]
\end{gathered}
$$

With our code called Candichangvar2F2, we can get all the candidates for the change of variables parameter $f$.

$>\mathrm{B}:=$ Candichangvar2F2(F1, R1, ext $)$;

$$
B:=\left[2 \frac{(x-7)^{4}(x-12)}{(x-3)^{2}},-2 \frac{(x-7)^{4}(x-12)}{(x-3)^{2}}\right]
$$

Our code called $f$ ind2F $21 n$ gives us the set of candidates for $\left[\left\{\left[a_{1}, a_{2}\right]\right\},\left\{\left[b_{1}, b_{2}\right]\right\}, f\right]$ (change of variable parameter and also upper and lower parameters of ${ }_{2} F_{2}$ )

$>$ find2F2ln $(\mathrm{L}, \mathrm{R} 1, \mathrm{~F} 1$, ext $, \mathrm{x}, \mathrm{t})$;

$$
\begin{aligned}
& {\left[\left\{\left[\frac{5}{18}, \frac{11}{126}\right],\left[\frac{11}{18}, \frac{53}{126}\right],\left[\frac{17}{18}, \frac{95}{126}\right]\right\},\left[1, \frac{11}{12}\right], 2 \frac{(x-7)^{4}(x-12)}{(x-3)^{2}}\right],} \\
& {\left[\left\{\left[-1, \frac{17}{21}\right],\left[1, \frac{17}{21}\right],\left[\frac{1}{3}, \frac{1}{7}\right],\left[\frac{2}{3}, \frac{10}{21}\right]\right\},\left[1, \frac{1}{12}\right], 2 \frac{(x-7)^{4}(x-12)}{(x-3)^{2}}\right],} \\
& {\left[\left\{\left[\frac{5}{18}, \frac{11}{126}\right],\left[\frac{11}{18}, \frac{53}{126}\right],\left[\frac{17}{18}, \frac{95}{126}\right]\right\},\left[1, \frac{11}{12}\right],-2 \frac{(x-7)^{4}(x-12)}{(x-3)^{2}}\right],} \\
& \left.\left[\left\{\left[-1, \frac{17}{21}\right],\left[1, \frac{17}{21}\right],\left[\frac{1}{3}, \frac{1}{7}\right],\left[\frac{2}{3}, \frac{10}{21}\right]\right\},\left[1, \frac{1}{12}\right],-2 \frac{(x-7)^{4}(x-12)}{(x-3)^{2}}\right]\right]
\end{aligned}
$$

Our implemented code Hyp2F2Solutions gives us all the transformation parameters, if they exist, and also the upper and lower parameters of ${ }_{2} F_{2}$ :

$>$ Hyp2F2Solutions (L);

$$
\left.\left\{\left[\left[\left[\frac{1}{7}, \frac{1}{3}\right],\left[1, \frac{1}{12}\right],[0],[1]\right]\right], 2 \frac{(x-12)(x-7)^{4}}{(x-3)^{2}}\right]\right\}
$$

Hence, L has ${ }_{2} F_{2}$ type solutions with hypergeometric parameters $a_{1}=1 / 3, a_{2}=1 / 7$, $b_{1}=1$ and $b_{2}=1 / 12$, and transformation parameters: $r=0, r_{0}=1, r_{1}=0, r_{2}=0$ and $f=\frac{2(x-7)^{4}(x-12)}{(x-3)^{2}}$.

\section{${ }_{1} F_{1}^{2}$ type solutions}

Let us consider the differential operator $\mathrm{L}_{11}^{2}$ associated to the ${ }_{1} F_{1}^{2}$ hypergeometric function with parameters (upper and lower) $a_{1}=1 / 7$ and $b_{1}=1 / 3$ :

$>$ L112:=MinOp (hypergeom $([1 / 7],[1 / 3], \mathrm{x}) \sim 2)$; 


$$
L 112:=D x^{3}+3 \frac{(-x+1 / 3)}{x} D x^{2}-x^{-2}\left(\frac{40}{21} x-2 x^{2}+\frac{1}{9}\right) D x-\frac{2}{7} \frac{-2 x-1 / 3}{x^{2}}
$$
by

Let us apply to $\mathrm{L}_{11}^{2}$ the change of variables transformation with parameter $f$ given

$>f:=(x-1) \sim 6 /(x-12) ;$

$$
f:=\frac{(x-1)^{6}}{x-12}
$$

That gives us the following operator called L:

$>$ L: $=$ ChangeOfVariables $($ L112,f $)$;

$$
\begin{aligned}
L:= & 63(x-12)^{5}(x-1)^{2}(5 x-71)^{2} D x^{3}-63\left(75 x^{8}-2580 x^{7}+29028 x^{6}-73447 x\right. \\
& \left.-124188 x^{5}+270570 x^{4}-334825 x^{3}+238286 x^{2}-74793\right)(x-12)^{3}(x-1) \\
& \times(5 x-71) D x^{2}+\left(41643716165 x^{2}+1536998515098 x^{4}-1230477210270 x^{9}\right. \\
& +460524395496 x^{10}-121187182692 x^{11}+21336285096 x^{12}-481721327852 x^{3} \\
& -2867623517288 x^{5}+3677984618849 x^{6}+2390095153908 x^{8}-3439319623344 x^{7} \\
& +78750 x^{16}-5418000 x^{15}+154148400 x^{14}-2357777520 x^{13}+124928729910 \\
& -26597677398 x)(x-12) D x+6\left(6 x^{6}-36 x^{5}+90 x^{4}-120 x^{3}+90 x^{2}-35 x-6\right) \\
& \times(x-1)^{5}(5 x-71)^{5}
\end{aligned}
$$

Let us assume that we have as input this operator $\mathrm{L}$ and then see how with our codes we can find its ${ }_{1} F_{1}^{2}$ type solutions if they exist. That means if we can find the hypergeometric parameters $a_{1}$ and $b_{1}$ of ${ }_{1} F_{1}^{2}$ and the transformation parameters such that

$$
\mathrm{L}_{11}^{2} \stackrel{f}{\longrightarrow}_{C} \mathrm{M} \longrightarrow_{E G} \mathrm{~L} .
$$

Let ext be the set of all non-rational numbers, parameters and names (except the variable $x$ ) which appear in the expression of the coefficients of $\mathrm{L}$ :

$>\operatorname{ext}:=\operatorname{indets}(\mathrm{L},\{\operatorname{Root} 0 f$, name $\}) \operatorname{minus}\{\mathrm{x}, \mathrm{Dx}\}$;

$$
\text { ext }:=\{\}
$$

Let extp be the set of all algebraic numbers given by the RootOf expressions in ext such that all its elements are algebraic over the minimal field containing $\mathbb{Q} \cup$ (ext $\backslash$ extp). Since sometimes we can use in our computations the RootOf expression associated with its index, we will take extp $=\{\}$.

Let us denote by $\mathrm{E}$ the set of all the singularities of $\mathrm{L}$ (we can find $\mathrm{E}$ using our implemented code called Singular).

$>\quad \mathrm{E}:=$ Singular $(\mathrm{L}, \mathrm{ext})$ :

Then the set of non-apparent singularities of $\mathrm{L}$ is given by

$>\mathrm{F}:=\operatorname{NotAppSing}(\mathrm{L}, \mathrm{E}$, ext $)$;

$$
F:=[[x-12,12],[\infty, \infty]]
$$

Let $S_{\text {irr }}$ be a set of irregular singularities of $\mathrm{L}$ which is the set of poles of $f$.

$$
\begin{aligned}
&>\operatorname{Sirr}:=\operatorname{irrsing} 1 \mathrm{~F} 1 \mathrm{sq}(\mathrm{L}, \mathrm{t}, \mathrm{F}, \mathrm{ext}) ; \\
& \operatorname{Sirr}:= {\left[[[x-12,12],[\infty, \infty]],\left[\left[\frac{2}{7},-1771561 t^{-1}+\frac{1}{3},-3543122 t^{-1}+\frac{8}{21}\right],\left[\frac{10}{7},\right.\right.\right.} \\
&-5 t^{-5}-12303 t^{-1}-24 t^{-4}-261 t^{-3}-2048 t^{-2}+\frac{5}{3},-10 t^{-5}-24606 t^{-1}
\end{aligned}
$$




$$
\begin{aligned}
& \left.\left.-48 t^{-4}-522 t^{-3}-4096 t^{-2}+\frac{40}{21}\right]\right],\left[\left[-3543122 t^{-1}+\frac{2}{21},-1771561 t^{-1}\right.\right. \\
& \left.+\frac{1}{21},-1771561 t^{-1}+\frac{1}{21}\right],\left[-10 t^{-5}-48 t^{-4}-522 t^{-3}-4096 t^{-2}+\frac{10}{21},\right. \\
& -24606 t^{-1},-5 t^{-5}-12303 t^{-1}-24 t^{-4}-261 t^{-3}-2048 t^{-2}+\frac{5}{21},-5 t^{-5} \\
& \left.\left.-12303 t^{-1}-24 t^{-4}-261 t^{-3}-2048 t^{-2}+\frac{5}{21}\right]\right],[1,5],\left[\left[\left[-3543122 t^{-1}\right.\right.\right. \\
& \left.+\frac{8}{21}, \frac{2}{7}\right],\left[-1771561 t^{-1}+\frac{1}{3}, \frac{2}{7}\right],\left[-3543122 t^{-1}+\frac{8}{21},-1771561 t^{-1}\right. \\
& \left.\left.+\frac{1}{3}\right]\right],\left[\left[-10 t^{-5}-24606 t^{-1}-48 t^{-4}-522 t^{-3}-4096 t^{-2}+\frac{40}{21}, \frac{10}{7}\right],\right. \\
& {\left[-5 t^{-5}-12303 t^{-1}-24 t^{-4}-261 t^{-3}-2048 t^{-2}+\frac{5}{3}, \frac{10}{7}\right],\left[-10 t^{-5}\right.} \\
& -24606 t^{-1}-48 t^{-4}-522 t^{-3}-4096 t^{-2}+\frac{40}{21},-5 t^{-5}-12303 t^{-1}-24 t^{-4} \\
& \left.\left.\left.\left.-261 t^{-3}-2048 t^{-2}+\frac{5}{3}\right]\right]\right],[1,1],\left[\left[\frac{2}{21}, \frac{1}{21}, \frac{1}{21}\right],\left[\frac{10}{21}, \frac{5}{21}, \frac{5}{21}\right]\right]\right]
\end{aligned}
$$

Let us take $\mathrm{G}$ as the set of elements in $\mathrm{F}$ at which $\mathrm{L}$ has all its exponent differences in $k$. Let $\mathrm{S}_{\text {reg }}$ be the set of elements in $\mathrm{G}$ at which $\mathrm{L}$ has logarithmic solutions or all its exponent differences are not, at same time, in $\mathbb{N}$. All the elements of $S_{\text {reg }}$ belong to the set of the zeroes of $f$, and they are given by

$>$ Sreg:=regsingtrue1F1sq (L, t, $\operatorname{Sirr}[-1]$, ext $)$;

$$
\text { Sreg }:=[]
$$

$\mathrm{S}_{\text {reg }}$ can be divided into two subsets: the set of non-removable singularities of $\mathrm{L}$ (at least one exponent difference is not an integer or L has logarithmic solution(s)) and a set of removable singularities of $\mathrm{L}$ (all the exponent differences are integers and $\mathrm{L}$ doesn't have logarithmic solution(s)). The set of non-removable singularities of L can also be divided into two sets: the singularities at which L has logarithmic solutions and the singularities at which L doesn't have logarithmic solutions. Hence, let us denote by $\mathrm{RS}_{\mathrm{reg}}$ a set of three sets: the first is the set of non-removable singularities of $\mathrm{L}$ with non-logarithmic solutions, the second is the set of removable singularities of $\mathrm{L}$ in $\mathrm{S}_{\mathrm{reg}}$, and the third is the set of singularities of $\mathrm{L}$ with logarithmic solutions. With our code called Sregseptrue1F1sq we can find this set $\mathrm{RS}_{\text {reg }}$ :

$>$ RSreg:= Sregseptrue1F1sq (L, Sreg, ext);

$$
\text { RSreg := [[], [], []] }
$$

Since $\mathrm{S}_{\mathrm{reg}}=[]$, we have absolutely no information about the zeroes of $f$ : that is the integer case.

In order to make our codes faster, we have implemented one procedure called IrrRegAppsing1F1sq which gives at the same time and, by avoiding duplicate computations, in a shorter time all the outputs of the procedures irrsing1F1sq, regsingtrue1F1sq and Sregseptrue1F1sq.

$>$ R1:=IrrRegAppsing1F1sq (L, t , E, ext ): 
With our code called Hyp1F1sqSubst, we can get the candidates for $f$ up to a constant (the polar part of $f$ ).

$>\mathrm{F} 1:=\operatorname{Hyp} 1 \mathrm{~F} 1 \mathrm{sqSubst}(\mathrm{L}, \mathrm{x}, \mathrm{t}, \mathrm{R} 1[1], \mathrm{ext})$;

$$
\begin{aligned}
F 1:= & {\left[-1771561(x-12)^{-1}-x^{5}-6 x^{4}-87 x^{3}-1024 x^{2}-12303 x,+1024 x^{2}\right.} \\
& -1771561(x-12)^{-1}+x^{5}+6 x^{4}+87 x^{3}+12303 x, 1771561(x-12)^{-1} \\
& -x^{5}-6 x^{4}-87 x^{3}-1024 x^{2}-12303 x, 1771561(x-12)^{-1}+x^{5}+87 x^{3} \\
& \left.+6 x^{4}+1024 x^{2}+12303 x\right]
\end{aligned}
$$

Our code called find1F1sqInt gives us the set of candidates for $\left[\left\{a_{1}\right\},\left\{b_{1}\right\}, f\right]$ (change of variable parameter and also upper and lower parameter of ${ }_{1} F_{1}^{2}$ )

$$
\begin{aligned}
& >\text { find1F1sqInt (L, R1, F1, } x, t, e x t) \text {; } \\
& {\left[\left[\left\{\frac{11}{28}, \frac{25}{28}, \frac{37}{84}, \frac{79}{84}\right\},\left[\frac{5}{6}\right],-\frac{(x-1)^{6}}{x-12}\right],\left[\left\{\frac{19}{84}, \frac{23}{84}, \frac{61}{84}, \frac{65}{84}\right\},\left[\frac{1}{2}\right],-\frac{(x-1)^{6}}{x-12}\right],\right.} \\
& {\left[\left\{\frac{5}{14}, \frac{6}{7}, \frac{13}{42}, \frac{17}{21}\right\},\left[\frac{2}{3}\right],-\frac{(x-1)^{6}}{x-12}\right],\left[\left\{\frac{3}{28}, \frac{5}{84}, \frac{17}{28}, \frac{47}{84}\right\},\left[\frac{1}{6}\right],-\frac{(x-1)^{6}}{x-12}\right],} \\
& {\left[\left\{\frac{1}{7}, \frac{4}{21}, \frac{9}{14}, \frac{29}{42}\right\},\left[\frac{1}{3}\right],-\frac{(x-1)^{6}}{x-12}\right],\left[\left\{\frac{11}{28}, \frac{25}{28}, \frac{37}{84}, \frac{79}{84}\right\},\left[\frac{5}{6}\right], \frac{(x-1)^{6}}{x-12}\right],} \\
& {\left[\left\{\frac{19}{84}, \frac{23}{84}, \frac{61}{84}, \frac{65}{84}\right\},\left[\frac{1}{2}\right], \frac{(x-1)^{6}}{x-12}\right],\left[\left\{\frac{5}{14}, \frac{6}{7}, \frac{13}{42}, \frac{17}{21}\right\},\left[\frac{2}{3}\right], \frac{(x-1)^{6}}{x-12}\right],} \\
& \left.\left[\left\{\frac{3}{28}, \frac{5}{84}, \frac{17}{28}, \frac{47}{84}\right\},\left[\frac{1}{6}\right], \frac{(x-1)^{6}}{x-12}\right],\left[\left\{\frac{1}{7}, \frac{4}{21}, \frac{9}{14}, \frac{29}{42}\right\},\left[\frac{1}{3}\right], \frac{(x-1)^{6}}{x-12}\right]\right]
\end{aligned}
$$

Our implemented code Hyp1F1sqSolutions gives us all the transformation parameters, if they exist, and also the upper and lower parameters of ${ }_{1} F_{1}^{2}$ :

$>$ Hyp1F1sqSolutions (L);

$$
\left.\left\{\left[\left[\left[\frac{1}{7}\right],\left[\frac{1}{3}\right],[0],[1]\right]\right], \frac{(x-1)^{6}}{x-12}\right]\right\}
$$

Hence, $\mathrm{L}$ has ${ }_{1} F_{1}^{2}$ type solutions with hypergeometric parameters $a_{1}=1 / 7$ and $b_{1}=1 / 3$, and transformation parameters: $r=0, r_{0}=1, r_{1}=0, r_{2}=0$ and $f=\frac{(x-1)^{6}}{(x-12)}$.

\section{$5.5{ }_{1} F_{2}$ and ${ }_{0} F_{2}$ type solutions}

Here the technique of resolutions is a little bit similar for the Bessel square root functions with the change of variable parameters $f \neq g^{2}$ where $g \in k(x)$. That is due to the form of exponent differences at 0 and $\infty$, and the fact that the ramification index of the generalized exponents of $\mathrm{L}_{0} \in\left\{\mathrm{L}_{12}, \mathrm{~L}_{02}\right\}$ at 0 is 1 and at $\infty$ belong to $\{2,3\}$, i.e. is greater than one.

We can assume $f=\frac{\mathrm{A}}{\mathrm{B}}$ with $\mathrm{A}, \mathrm{B} \in k[x], \mathrm{B}$ monic and $\operatorname{gcd}(\mathrm{A}, \mathrm{B})=1$ since we have some generalized exponents of $\mathrm{L}_{0} \in\left\{\mathrm{L}_{12}, \mathrm{~L}_{02}\right\}$ at $\infty$ with ramification index different from 1. 
The problem now is how to get information about A and B from L (our input operator).

\subsubsection{Truncated series for $f$}

Let $\mathrm{L}_{0} \in\left\{\mathrm{L}_{12}, \mathrm{~L}_{02}\right\}$ with $n_{e} \in\{2,3\}$ its ramification index at $\infty$. We know all the poles of $f$, but the exponent differences give us just information about the polar part of $f^{1 / n_{e}}$.

The following lemma, definition and remark are similar to Lemma 4.15, Definition 4.16 and Remark 4.17 in the Bessel square root case:

Lemma 5.10. Let $p$ be a point in $k$ and $n_{e} \in\{2,3\}$. If $f \in k(x)$ and $f^{1 / n_{e}}=\sum_{i} a_{i} t_{p}^{i}$, where $i \in \frac{1}{n_{e}} \mathbb{Z}, a_{i} \in k$ and $t_{p}$ is the local parameter at $x=p$, then the set $\left\{i \mid a_{i} \neq 0\right\}$ is either a subset of $\mathbb{Z}$ or a subset of $\frac{1}{n_{e}}+\mathbb{Z}$.

Proof. Similar to the proof of lemma 4.15.

Definition 5.11. Let $p \in k$ and $f^{1 / n_{e}}=\sum_{i=N}^{\infty} a_{i} t_{p}^{i / n_{e}}, a_{i} \in k$ and $a_{N} \neq 0$. We say that we have an $n$-term truncated power series for $f^{1 / n_{e}}$ if the coefficients of $t_{p}^{N / 2}, \ldots, t_{p}^{(N+n-1) / n_{e}}$ are known.

Remark 5.12. If an n-term truncated series for $f^{1 / n_{e}}$ is known, then we can compute an $n$-term truncated series for $f$.

We have implemented in Maple one algorithm called Sirr1F2info1 when $\mathrm{L}_{0}=\mathrm{L}_{12}$ and another one called Sirr0F2info1 when $\mathrm{L}_{0}=\mathrm{L}_{02}$, to find all those truncated series for $f$ related to the elements of $\mathrm{S}_{\mathrm{irr}}(\mathrm{L})$.

\subsubsection{How to compute the denominator B of $f$}

With our implemented algorithms irrsing1F2 and irrsing0F2 we can get the multiplicity order $m_{p}$ of each pole $p$ of $f$ (elements of $\mathrm{S}_{\mathrm{irr}}(\mathrm{L})$ ). To retrieve B we just use Lemma 4.18 for the Bessel square root case:

$$
\mathrm{B}=\prod_{p \in \mathrm{S}_{\mathrm{irr}}(\mathrm{L})}(x-p)^{m_{p}}=\prod_{p \in \mathrm{S}_{\mathrm{irr}}(\mathrm{L})} t_{p}^{m_{p}}=\prod_{P_{s} \in \overline{\mathrm{S}_{\mathrm{irr}}(\mathrm{L})} \backslash\{1\}} P_{s}^{m_{s}}
$$

Our implemented algorithms Sirr1F2info1 when $\mathrm{L}_{0}=\mathrm{L}_{12}$ or Sirr0F2info1 when $\mathrm{L}_{0}=\mathrm{L}_{02}$ compute this denominator $\mathrm{B}$ of $f$.

\subsubsection{How to get a bound for the degree of the numerator A of $f$}

As for the Bessel square root case, Remarks 4.19, Lemma 4.20 and Corollary 4.21 also hold here:

$$
d_{\mathrm{A}}=\sum_{s \in \mathrm{S}_{\mathrm{irr}}(\mathrm{L})} m_{s}
$$

Our implemented algorithms Sirr1F2info1 when $\mathrm{L}_{0}=\mathrm{L}_{12}$ or Sirr0F2info1 when $\mathrm{L}_{0}=\mathrm{L}_{02}$ compute $d_{\mathrm{A}}$. 


\subsubsection{How to get linear equations for the coefficients of the nu- merator A of $f$}

As for the Bessel square root case, Lemma 4.22 and Remarks 4.23 hold also here. Concerning Lemma 4.24, here we have

Lemma 5.13. For $s \in \mathrm{S}_{\text {irr }}(\mathrm{L})$ let $m_{s}$ be its multiplicity order as a zero of $f, g_{s}$ the polar part of $f$ at $s$ and $\bar{g}_{s}$ the $\left\lceil m_{s} / n_{e}\right\rceil$-truncated series of $f$ at $s$. Let

$$
u(x)=\left[f-\sum_{P_{s} \in \overline{\mathrm{S}_{i r r}(\mathrm{~L})}} \bar{g}_{s}\right] \cdot \prod_{P_{s} \in \overline{\mathrm{S}_{i r r}(\mathrm{~L})} \backslash\{1\}} P_{s}^{\left\lfloor m_{s} *\left(n_{e}-1\right) / n_{e}\right\rfloor} .
$$

Then $u(x) \in k[x]$ and the remainder of the Euclidean division of numer $(u(x))$ by denom $(u(x))$ will give us $\sum_{P_{s} \in \overline{\mathrm{S}_{\text {irr }}(\mathrm{L}) \backslash\{1\}}} \operatorname{deg}\left(P_{s}\right) \cdot\left\lceil m_{s} / n_{e}\right\rceil$ linear equations for the coefficients of $\mathrm{A}$.

Furthermore, if $\infty \in \mathrm{S}_{\text {irr }}(\mathrm{L})$, the quotient of the Euclidean division of numer $(u(x))$ by denom $(u(x))$ will give us, in addition, $\left\lceil m_{\infty} / n_{e}\right\rceil$ linear equations.

Proof. Similar to the proof of Lemma 4.24.

Similar as in Remarks 4.25, here we have

Remarks 5.14. 1. We know

$$
\sum_{P_{s} \in \overline{\mathrm{S}_{r e g}(\mathrm{~L})}} \operatorname{deg}\left(P_{s}\right)+\sum_{P_{s} \in \overline{\mathrm{S}_{i r r}(\mathrm{~L})}} \operatorname{deg}\left(P_{s}\right) \cdot\left\lceil m_{s} / n_{e}\right\rceil \text { linear equations for the coefficients of }
$$

A where $m_{s}$ is the multiplicity order of $s \in \mathrm{S}_{\text {irr }}(\mathrm{L})$ as a pole of $f$. That means we have

$$
\sum_{s \in \mathrm{S}_{\text {reg }}(\mathrm{L})} 1+\sum_{s \in \mathrm{S}_{\text {irr }}(\mathrm{L})}\left\lceil m_{s} / n_{e}\right\rceil \text { linear equations. }
$$

2. Since $\mathrm{S}_{r e g}(\mathrm{~L})$ can be an empty set, but $\mathrm{S}_{\text {irr }}(\mathrm{L})$ not, we know at least

$$
\sum_{P_{s} \in \overline{\mathrm{S}_{i r r}(\mathrm{~L})}} \operatorname{deg}\left(P_{s}\right) \cdot\left\lceil m_{s} / n_{e}\right\rceil \text { linear equations for the coefficients of } \mathrm{A} \text {. }
$$

That means we have

$$
\sum_{s \in \mathrm{S}_{i r r}(\mathrm{~L})}\left\lceil m_{s} / n_{e}\right\rceil \text { linear equations. }
$$

Similar as in Lemma 4.26, here we have

Lemma 5.15. The number of linear equations for the coefficients of $\mathrm{A}$ is greater or equal to $\frac{1}{n_{e}} d_{\mathrm{A}}+\sum_{p \in \mathrm{S}_{r e g}(\mathrm{~L})} 1$.

Proof. Similar to the proof of Lemma 4.26. 


\subsubsection{How to compute the upper parameter $a_{1}$ when $\mathrm{L}_{0}=\mathrm{L}_{12}$}

Let us assume that we know $b_{1}$ and $b_{2}$. To find candidates for $\left\{a_{1}\right\}$, we proceed as follows:

1. We take a candidate $\left\{b_{1}, b_{2}\right\}$ for the lower parameter(s) of ${ }_{1} F_{2}$.

2. Let $p \in \mathrm{S}_{\text {irr }}(\mathrm{L})$ with $m_{p}$ its multiplicity order as a pole of $f$.

(a) We choose the two exponent differences of L at $p$ which have the same, up to a factor -1 and modulo $\frac{1}{2} \mathbb{Z}$, non-constant part. Let $\mathrm{C}_{p}^{1}$ and $\mathrm{C}_{p}^{2}$ be the constant part of those exponent differences:

$$
\left\{\begin{array}{l}
\mathrm{C}_{p}^{1}= \pm \frac{m_{p}}{2}\left(b_{1}+b_{2}-3 a_{1}-\frac{1}{2}\right) \text { modulo } \frac{1}{2} \mathbb{Z} \\
\mathrm{C}_{p}^{2}= \pm \frac{m_{p}}{2}\left(b_{1}+b_{2}-3 a_{1}-\frac{1}{2}\right) \text { modulo } \frac{1}{2} \mathbb{Z}
\end{array}\right.
$$

(b) We compute the set $\mathrm{N}_{p}$ of candidates modulo $\mathbb{Z}$ for $\pm a_{1}$ associated to $p$

$$
\mathrm{N}_{p}=\left\{\left(b_{1}+b_{2}-\frac{1}{2}-\frac{j \pm 2 \mathrm{C}_{p}^{1}}{m_{p}}\right) \cdot \frac{1}{3}|j \in \mathbb{Z},| j \mid \leq 3 m_{p}-1\right\}
$$

We replace in $\mathrm{N}_{p}$ all the rational parts of elements by their representant modulo $\mathbb{Z}$ in $[0,1]$.

3. $\mathrm{E}=\bigcap_{p \in \mathrm{S}_{\mathrm{irr}}(\mathrm{L})} \mathrm{N}_{p}$ is the set of candidates modulo $\mathbb{Z}$ for $\pm a_{1}$. If $\mathrm{E}=\emptyset$ then $a_{1}$ doesn't exist. That means $\left\{b_{1}, b_{2}\right\}$ is not a good candidate for the lower parameters of ${ }_{1} F_{2}$. We take another $\left\{b_{1}, b_{2}\right\}$ and repeat the process.

We have implemented in Maple an algorithm called findcandai1F2 to find candidates modulo $\mathbb{Z}$ for the upper parameter of ${ }_{1} F_{2}$.

\subsubsection{How to compute the numerator A of $f$}

As for the Bessel square root case, we also have here four cases:

- "Easy case": as in Bessel square root case;

- "Logarithmic case": when $b_{1} \in \mathbb{Z}$ or $b_{2} \in \mathbb{Z}$ or $b_{1}-b_{2} \in \mathbb{Z}$ or $\left(2 b_{1}, 2 b_{2} \in \mathbb{Z}\right.$ with $b 1 \cdot b 2<0)$

- "Irrational case": when we are not in the logarithmic case and $b_{1}$ or $b_{2}$ is not a rational number;

- "Rational case": when we are not in the logarithmic case and $b_{1}, b_{2} \in \mathbb{Q}$. 


\section{Easy Case}

To find candidates for $\left[\left\{a_{1}\right\},\left\{b_{1}, b_{2}\right\}, f\right]$ when $\mathrm{L}_{0}=\mathrm{L}_{12}$ or candidates for $\left[\left\{b_{1}, b_{2}\right\}, f\right]$ when $\mathrm{L}_{0}=\mathrm{L}_{02}$, we proceed as follows:

1. We solve our linear equations for the coefficients of A using Lemma 4.22 and Lemma 5.13:

(a) If we find solutions, then we can get $\mathrm{A}$ and therefore $f=\mathrm{A} / \mathrm{B}$ because we know how to find B.

(b) If we don't find solutions then we cannot find ${ }_{p} F_{q} \in\left\{{ }_{1} F_{2},{ }_{0} F_{2}\right\}$ type solutions for L using the Easy case. That means the number of linear equations for the coefficients of $\mathrm{A}$ is less than or equal to $d_{\mathrm{A}}$, or the system of equations given by those coefficients doesn't have solutions.

2. We compute the set $\mathrm{C}$ of candidates for $\left\{b_{1}, b_{2}\right\}$ (we have shown above how to get it).

3. If $\mathrm{L}_{0}=\mathrm{L}_{12}$ then for $\left\{b_{1}, b_{2}\right\} \in \mathrm{C}$

(a) we compute $\mathrm{C}_{\left(b_{1}, b_{2}\right)}$ the set of candidates for $a_{1}$ related to $\left\{b_{1}, b_{2}\right\}$ (we have shown that above).

(b) If $\mathrm{C}_{\left(b_{1}, b_{2}\right)}=\emptyset$ then we will go back and take another $\left\{b_{1}, b_{2}\right\}$ in $\mathrm{C}$.

All the lists $\left[\mathrm{C}_{\left(b_{1}, b_{2}\right)},\left\{b_{1}, b_{2}\right\}, f\right]$ for which $\mathrm{C}_{\left(b_{1}, b_{2}\right)} \neq \emptyset$ generate a set called $\mathrm{E}$.

4. If $\mathrm{L}_{0}=\mathrm{L}_{02}$ then $\mathrm{E}=\{\mathrm{C}, f\}$.

5. If $\mathrm{E} \neq \emptyset$ then $\mathrm{L}$ has $F_{0} \in\left\{{ }_{1} F_{2},{ }_{0} F_{2}\right\}$ type solutions, if not we cannot solve $\mathrm{L}$ in terms of $F_{0} \in\left\{{ }_{1} F_{2},{ }_{0} F_{2}\right\}$ functions.

We have implemented in Maple, for this case, one algorithm called easy1F2 to find candidates for $\left[\left\{a_{1}\right\},\left\{b_{1}, b_{2}\right\}, f\right]$ when $\mathrm{L}_{0}=\mathrm{L}_{12}$ and another one called easy0F2 to find candidates for $\left[\left\{b_{1}, b_{2}\right\}, f\right]$ when $\mathrm{L}_{0}=\mathrm{L}_{02}$.

\section{Logarithmic Case}

The technique to find candidates for $f$ is the same as for the Bessel square root case.

Once we get candidates for $f=\mathrm{A} / \mathrm{B}$, we proceed as follows to find the candidates for the upper and lower parameters for $F_{0} \in\left\{{ }_{1} F_{2},{ }_{0} F_{2}\right\}$ related to any candidate for $f$ :

1. Let $\mathrm{F}$ be the set of candidates for $f$.

2. for $g \in \mathrm{F}$

(a) We compute the set $\mathrm{C}$ of candidates for $\left\{b_{1}, b_{2}\right\}$ (we have shown above how to get it).

(b) If $\mathrm{L}_{0}=\mathrm{L}_{12}$ then for $\left\{b_{1}, b_{2}\right\} \in \mathrm{C}$

i. we compute $\mathrm{C}_{\left(b_{1}, b_{2}\right)}$ the set of candidates for $a_{1}$ (we have shown above how to get it)

ii. If $\mathrm{C}_{\left(b_{1}, b_{2}\right)}=\emptyset$ then we will return and take another $\left\{b_{1}, b_{2}\right\}$ in $\mathrm{C}$. 
All the lists $\left[\mathrm{C}_{\left(b_{1}, b_{2}\right)},\left\{b_{1}, b_{2}\right\}, f\right]$ for which $\mathrm{C}_{\left(b_{1}, b_{2}\right)} \neq \emptyset$ generate a set called $\mathrm{E}$. (c) If $\mathrm{L}_{0}=\mathrm{L}_{02}$ then $\mathrm{E}=[\mathrm{C}, f]$.

3. If $\mathrm{E} \neq \emptyset$ then $\mathrm{L}$ has $F_{0} \in\left\{{ }_{1} F_{2},{ }_{0} F_{2}\right\}$ type solutions, if not, we cannot solve $\mathrm{L}$ in terms of $F_{0} \in\left\{{ }_{1} F_{2},{ }_{0} F_{2}\right\}$ functions.

We have implemented in Maple, for this case, one algorithm called find1F2ln to find candidates for $\left[\left\{a_{1}\right\},\left\{b_{1}, b_{2}\right\}, f\right]$ when $\mathrm{L}_{0}=\mathrm{L}_{12}$ and another one called $\mathrm{f}$ ind0F2ln to find candidates for $\left[\left\{b_{1}, b_{2}\right\}, f\right]$ when $\mathrm{L}_{0}=\mathrm{L}_{02}$.

\section{Irrational Case}

The technique to find candidates for $f$ is a little bit similar as for the Bessel square root case (just some modifications).

Once we get candidates for $f=\mathrm{A} / \mathrm{B}$, we use the same technique as in the logarithmic case to find the candidates for the upper and lower parameters for $F_{0} \in\left\{{ }_{1} F_{2},{ }_{0} F_{2}\right\}$ related to any candidate for $f$.

We have implemented in Maple, for this case, one algorithm called find1F2Irr to find candidates for $\left[\left\{a_{1}\right\},\left\{b_{1}, b_{2}\right\}, f\right]$ when $\mathrm{L}_{0}=\mathrm{L}_{12}$ and another one called find0F2Irr to find candidates for $\left[\left\{b_{1}, b_{2}\right\}, f\right]$ when $\mathrm{L}_{0}=\mathrm{L}_{02}$.

\section{Rational Case}

Here the numerator $\mathrm{A}$ of $f$ can be written in the form $\mathrm{A}=c \mathrm{~A}_{1} \mathrm{~A}_{2}^{d}$ as in Corollary 4.34.

The technique to find candidates for $f$ is similar as for the Bessel square root case with $f \neq g^{2}$ where $g \in k(x)$.

Once we get candidates for $f=\mathrm{A} / \mathrm{B}=\frac{c \mathrm{~A}_{1} \mathrm{~A}_{2}^{d}}{\mathrm{~B}}$, we use the same technique as in the logarithmic case to find the candidates for the upper and lower parameters for $F_{0} \in$ $\left\{{ }_{1} F_{2},{ }_{0} F_{2}\right\}$ related to any candidate for $f$.

We have implemented in Maple, for this case, one algorithm called find1F2Rat to find candidates for $\left[\left\{a_{1}\right\},\left\{b_{1}, b_{2}\right\}, f\right]$ when $\mathrm{L}_{0}=\mathrm{L}_{12}$ and another one called find0F2Rat to find candidates for $\left[\left\{b_{1}, b_{2}\right\}, f\right]$ when $\mathrm{L}_{0}=\mathrm{L}_{02}$.

\subsubsection{Examples}

We have written some algorithms on how to find $F_{0} \in\left\{{ }_{1} F_{2},{ }_{0} F_{2}\right\}$ type solutions if they exist. We have also summarize them by writing other algorithms called Hyp1F2Solutions when $\mathrm{L}_{0}=\mathrm{L}_{12}$ and HypOF2Solutions when $\mathrm{L}_{0}=\mathrm{L}_{02}$, which take as input a third-order linear differential operator L and return, if they exist, all the parameters of transformations $\left(r, r_{2}, r_{1}, r_{0}, f \in k(x)\right)$ and also the upper and lower parameter(s) of $F_{0} \in\left\{{ }_{1} F_{2},{ }_{0} F_{2}\right\}$ such that we are in situation (5.1)

$$
\mathrm{L}_{0} \stackrel{f}{\longrightarrow}_{C} \mathrm{M} \longrightarrow \longrightarrow_{E G} \mathrm{~L}
$$

with $\mathrm{L}_{0} \in\left\{\mathrm{L}_{12}, \mathrm{~L}_{02}\right\}$. If not, it will return "No $F_{0}$ type solutions". Those algorithms deal with all the cases and give us short solutions, if they exist, associated to the upper and lower parameter(s) of $F_{0}$.

We will take here just one example, depending on $F_{0} \in\left\{{ }_{1} F_{2},{ }_{0} F_{2}\right\}$, and show explicitly this algorithm works. 


\section{${ }_{1} F_{2}$ type solutions}

Let us consider the differential operator $\mathrm{L}_{12}$ associated to the ${ }_{1} F_{2}$ hypergeometric function with parameters (upper and lower) $a_{1}=1 / 2, b_{1}=1 / 3$ and $b_{2}=\operatorname{RootOf}\left(x^{2}+1\right)$. To get $\mathrm{L}_{12}$ with Maple, we proceed as follows:

$$
\begin{aligned}
& >\text { eq:=sumdiffeq(hyperterm([a1], [b1,b2],x,i), i, J(x)); } \\
& e q:=\left(\frac{d^{3}}{d x^{3}} J(x)\right) x^{2}+(b 2+1+b 1)\left(\frac{d^{2}}{d x^{2}} J(x)\right) x+(b 2 b 1-x) \frac{d}{d x} J(x)-a 1 J(x)=0 \\
& >\text { LA: }=\operatorname{de} 2 \operatorname{diffop}(\text { eq }, \mathrm{J}(\mathrm{x})) \text {; } \\
& L A:=x^{2} D x^{3}+(x b 2+x+x b 1) D x^{2}+(b 2 b 1-x) D x-a 1 \\
& >\operatorname{L12}:=\operatorname{subs}\left(\left\{\mathrm{a} 1=1 / 2, \mathrm{~b} 1=1 / 3, \mathrm{~b} 2=\operatorname{RootO} f\left(\mathrm{x}^{\wedge} 2+1\right)\right\}, \mathrm{LA}\right) \text {; } \\
& L 12:=x^{2} D x^{3}+\left(x \operatorname{RootOf}\left({ }_{-} Z^{2}+1\right)+\frac{4}{3} x\right) D x^{2}+\left(\frac{1}{3} \operatorname{RootOf}\left({ }_{-} Z^{2}+1\right)-x\right) D x-\frac{1}{2}
\end{aligned}
$$

Let us apply to $\mathrm{L}_{12}$ the change of variables transformation with parameter $f$ given by

$>f:=(2 *(x-1)-3) /((x-9)) ;$

$$
f:=2 \frac{(x-1)^{3}}{x-9}
$$

That gives us the following operator called L:

$$
\begin{aligned}
>\mathrm{L}:= & \text { ChangeOfVariables }(\mathrm{L} 12, \mathrm{f}) ; \\
L:= & 3(x-1)^{2}(x-13)^{2}(x-9)^{4} D x^{3}+\frac{1}{37}\left(6 \operatorname{RootOf}\left({ }_{-} Z^{2}+1\right)-1\right)(-962 x \\
& \left.+37 x^{2}+6685+2592 \operatorname{RootOf}\left({ }_{-} Z^{2}+1\right)\right)(x-13)(x-1)(x-9)^{3} D x^{2} \\
& -(x-9)\left(+24 x^{7}-952146 \operatorname{RootOf}\left(Z^{2}+1\right)+3252 \operatorname{RootOf}\left(Z^{2}+1\right) x^{3}\right. \\
& -50847+28151 x^{5}+2 \operatorname{RootOf}\left({ }_{-} Z^{2}+1\right) x^{5}-122 \operatorname{RootOf}\left(Z^{2}+1\right) x^{4} \\
& +2046851 x-45908 \operatorname{RootOf}\left(Z^{2}+1\right) x^{2}+331370 x \operatorname{RootOf}\left(Z^{2}+1\right) \\
& \left.-2683358 x^{2}+1390494 x^{3}-287627 x^{4}-1320 x^{6}\right) D x-24(x-13)^{5}(x-1)^{2}
\end{aligned}
$$

Let us assume that we have as input this operator $\mathrm{L}$ and then see how with our codes we can find its ${ }_{1} F_{2}$ type solutions if they exist. That means if we can find the hypergeometric parameters $a_{1}, b_{1}$ and $b_{2}$ of ${ }_{1} F_{2}$ and the transformation parameters such that

$$
\mathrm{L}_{12} \stackrel{f}{\longrightarrow}_{C} \mathrm{M} \longrightarrow_{E G} \mathrm{~L} .
$$

Let ext be the set of all non-rational numbers, parameters and names (except the variable $x$ ) which appear in the expression of the coefficients of $\mathrm{L}$ :

$$
\begin{array}{r}
>\operatorname{ext}:=\operatorname{indets}(\mathrm{L},\{\operatorname{RootOf}, \text { name }\}) \operatorname{minus}\{\mathrm{x}, \mathrm{Dx}\} ; \\
\operatorname{ext}:=\left\{\operatorname{RootOf}\left({ }_{-} Z^{2}+1\right)\right\}
\end{array}
$$

Let extp be the set of all algebraic numbers given by the RootOf expressions in ext such that all its elements are algebraic over the minimal field containing $\mathbb{Q} \cup$ (ext $\backslash$ extp). Since sometimes we can use in our computations the RootOf expression associated with its index, we will take extp $=\{\}$.

Let us denote by $\mathrm{E}$ the set of all the singularities of $\mathrm{L}$ (we can find $\mathrm{E}$ using our implemented code called Singular).

$>\quad E:=$ Singular $(\mathrm{L}$, ext $)$ : 
Then the set of non-apparent singularities of L is given by

$>\mathrm{F}:=\operatorname{NotAppSing}(\mathrm{L}, \mathrm{E}$, ext $)$;

$$
F:=[[\infty, \infty],[x-1,1],[x-9,9]]
$$

Let $\mathrm{S}_{\mathrm{irr}}$ be the set of irregular singularities of $\mathrm{L}$ which is the set of poles of $f$.

$>$ Sirr:=irrsing1F2(L, t, F, ext);

$$
\begin{aligned}
& \operatorname{Sirr}:=\left[[[\infty, \infty],[x-9,9]],\left[\left[1,2 \frac{\sqrt{2}}{t}-\frac{2}{3}+\operatorname{RootOf}\left({ }_{-} Z^{2}+1\right),-2 \frac{\sqrt{2}}{t}-\frac{2}{3}\right.\right.\right. \\
& \left.+\operatorname{RootOf}\left(Z_{-} Z^{2}+1\right)\right],\left[\frac{1}{2}, 32(\sqrt{t})^{-1}-\frac{1}{3}+1 / 2 \operatorname{RootOf}\left(Z^{2}+1\right),\right. \\
& \left.\left.-32(\sqrt{t})^{-1}-\frac{1}{3}+\frac{1}{2} \operatorname{RootOf}\left({ }_{-} Z^{2}+1\right)\right]\right],\left[\left[\frac{5}{3}-\operatorname{RootOf}\left({ }_{-} Z^{2}+1\right)\right.\right. \\
& -\frac{\operatorname{RootOf}\left(-8+{ }_{-} Z^{2}, \text { index }=1\right)}{t}, \frac{5}{3}-\frac{\operatorname{RootOf}\left(-8+{ }_{-} Z^{2}, \text { index }=2\right)}{t} \\
& \left.-\operatorname{RootOf}\left({ }_{-} Z^{2}+1\right),-2 \frac{\operatorname{RootOf}\left(-8+{ }_{-} Z^{2}\right)}{t}\right],\left[32(\sqrt{t})^{-1}-\frac{5}{6}\right. \\
& +\frac{1}{2} \operatorname{RootOf}\left(Z_{-} Z^{2}+1\right),-32(\sqrt{t})^{-1}-\frac{5}{6}+1 / 2 \operatorname{RootOf}\left(Z^{2}+1\right), \\
& \left.\left.\left.-64(\sqrt{t})^{-1}\right]\right],\left[1, \frac{1}{2}\right]\right]
\end{aligned}
$$

Let us take $\mathrm{G}$ as the set of elements in $\mathrm{F}$ at which $\mathrm{L}$ has all its exponent differences in $k$. Let $\mathrm{S}_{\text {reg }}$ be the set of elements in $\mathrm{G}$ at which $\mathrm{L}$ has logarithmic solutions or all its exponent differences are not, at same time, in $\mathbb{N}$. All the elements of $S_{\text {reg }}$ belong to the set of the zeroes of $f$ and they are given by

$$
\begin{aligned}
&>\text { Sreg: }=\text { regsingtrue1F2 }(\mathrm{L}, \mathrm{t}, \operatorname{Sirr}[-1], \text { ext }) ; \\
& \text { Sreg }:=\left[[[x-1,1]],\left[\left[0,2,3-3 \operatorname{RootOf}\left({ }_{-} Z^{2}+1\right)\right]\right],\left[\left[2,3-3 \operatorname{RootOf}\left({ }_{-} Z^{2}+1\right),\right.\right.\right. \\
&\left.\left.\left.1-3 \operatorname{RootOf}\left({ }_{-} Z^{2}+1\right)\right]\right]\right]
\end{aligned}
$$

$\mathrm{S}_{\text {reg }}$ can be divided into two subsets: the set of non-removable singularities of $\mathrm{L}$ (at least one exponent difference is not an integer or L has logarithmic solution(s)) and a set of removable singularities of $\mathrm{L}$ (all the exponent differences are integers and $\mathrm{L}$ doesn't have logarithmic solution(s)). The set of non-removable singularities of L can also be divided into two sets: the singularities at which L has logarithmic solutions and the singularities at which L doesn't have logarithmic solutions. Hence, let us denote by $\mathrm{RS}_{\mathrm{reg}}$ a set of three sets: the first is the set of non-removable singularities of $\mathrm{L}$ with non-logarithmic solutions, the second is the set of removable singularities of $\mathrm{L}$ in $\mathrm{S}_{\text {reg, }}$, and the third is the set of singularities of $\mathrm{L}$ with logarithmic solutions. With our code called Sregseptrue1F2 we can find this set $\mathrm{RS}_{\text {reg: }}$ :

$>$ RSreg:= Sregseptrue1F2(L, Sreg, ext);

$$
\begin{aligned}
\text { RSreg: }=[[[[x-1,1]],[ & {\left.\left[0,2,3-3 \operatorname{RootOf}\left({ }_{-} Z^{2}+1\right)\right]\right],\left[\left[\left[3-3 \operatorname{RootOf}\left({ }_{-} Z^{2}+1\right),\right.\right.\right.} \\
& \left.\left.\left.\left.\left.1-3 \operatorname{RootOf}\left({ }_{-} Z^{2}+1\right)\right],[2]\right]\right]\right],[],[]\right]
\end{aligned}
$$

Since $\mathrm{RS}_{\mathrm{reg}}[1] \neq[]$, we know some zeroes of $f$. Those zeroes represent all the zeroes of $f$ because the exponent differences of $\mathrm{L}$ at elements of $\mathrm{RS}_{\text {reg }}[1]$ are not in $\mathbb{Q}$.

In order to make our codes faster, we have implemented one procedure called 
IrrRegAppsing1F2 which gives at the same time and, by avoiding duplicate computations, in a shorter time all the outputs of the procedures irrsing1F2, regsingtrue1F2 and Sregseptrue1F2.

\section{$>\mathrm{R} 1:=\operatorname{Irr} R e g A p p s i n g 1 F 2(\mathrm{~L}, \mathrm{t}, \mathrm{E}$, ext $):$}

Let $f=A / B$ with $\mathrm{A}$ and $\mathrm{B}$ as in our theoretical part, and $l=1$. We can get the truncated series for $f$ related to the elements of $\mathrm{S}_{\text {irr }}$, the degree of $\mathrm{A}$ denoted $d_{\mathrm{A}}$ and also the number $n$ of linear equations satisfied by the coefficients of $\mathrm{A}$ using our implemented code called Sirr1F2info1. It shows us if we are in the "Easy case" or not. If we are not in this case, we can see in which case we are according to the exponent differences of elements in $\mathrm{RS}_{\mathrm{reg}}[1]$.

$$
\begin{aligned}
& >\text { info1:= Sirr1F2info1(L,R1[1],R1[2], 1, x, t, ext); } \\
& \text { info1 := }\left[\left[\left[9, x-9,\left[1024(x-9)^{-1}\right], 1,\{\},\left\{\operatorname{RootOf}\left({ }_{-} Z^{2}+1\right)\right\}\right],\left[\infty, x^{-1},\left[2 x^{2}\right]\right. \text {, }\right.\right. \\
& \left.\left.1,\left\{\operatorname{RootOf}\left({ }_{-} Z^{2}-2\right)\right\},\left\{\operatorname{RootOf}\left({ }_{-} Z^{2}-2\right), \operatorname{RootOf}\left({ }_{-} Z^{2}+1\right)\right\}\right]\right] \text {, } \\
& 3,3, x-9,1]
\end{aligned}
$$

We have $n=3$ and $d_{\mathrm{A}}=3$. Hence $n=d_{\mathrm{A}}$ and therefore we cannot solve those equations and get A: that is not the "Easy case".

Since the exponent differences of $L$ at elements of $\mathrm{RS}_{\mathrm{reg}}[1]$ are not in $\mathbb{Q}$, we are in the irrational case. Our code called find1F2Irr gives us the set of candidates for $\left[\left\{a_{1}\right\},\left\{\left[b_{1}, b_{2}\right]\right\}, f\right]$ (change of variable parameter and also upper and lower parameters of ${ }_{1} F_{2}$ )

$$
\begin{aligned}
& >\text { find } 1 F 2 \operatorname{Irr}(L, R 1 \text {, info1, } x, t, \text { ext }) \text {; } \\
& {\left[\left[\left[\left\{\frac{1}{18}, \frac{7}{18}, \frac{13}{18}\right\},\left[\frac{2}{3}, \frac{1}{3}+\operatorname{RootOf}\left(Z_{-}^{2}+1\right)\right]\right],\left[\left\{\frac{1}{2}, \frac{1}{6}, \frac{5}{6}\right\},\left[\frac{2}{3}, \frac{2}{3}\right.\right.\right.\right.} \\
& \left.\left.+\operatorname{RootOf}\left(Z^{2}+1\right)\right]\right],\left[\left\{\frac{1}{2}, \frac{1}{6}, \frac{5}{6}\right\},\left[\frac{1}{3}, 1+\operatorname{RootOf}\left(Z^{2}+1\right)\right]\right], \\
& {\left[\left\{\frac{1}{2}, \frac{1}{6}, \frac{5}{6}\right\},\left[\frac{1}{3}, \operatorname{RootOf}\left(Z^{2}+1\right)\right]\right],\left[\left\{\frac{5}{18}, \frac{11}{18}, \frac{17}{18}\right\},\left[\frac{2}{3}, \operatorname{RootOf}\left(Z^{2}+1\right)\right]\right],} \\
& {\left[\left\{\frac{5}{18}, \frac{11}{18}, \frac{17}{18}\right\},\left[\frac{2}{3}, 1+\operatorname{RootOf}\left(Z^{2}+1\right)\right]\right],\left[\left\{\frac{5}{18}, \frac{11}{18}, \frac{17}{18}\right\},\left[\frac{1}{3}, \frac{1}{3}\right.\right.} \\
& \left.\left.+\operatorname{RootOf}\left(Z^{2}+1\right)\right]\right],\left[\left\{\frac{1}{18}, \frac{7}{18}, \frac{13}{18}\right\},\left[\frac{1}{3}, \frac{2}{3}+\operatorname{RootOf}\left(Z^{2}+1\right)\right]\right] \text {, } \\
& \left.2 \frac{(x-1)^{3}}{x-9}\right],\left[\left[\left\{\frac{1}{18}, \frac{7}{18}, \frac{13}{18}\right\},\left[\frac{2}{3}, \frac{1}{3}+\operatorname{RootOf}\left(Z^{2}+1\right)\right]\right],\left[\left\{\frac{1}{2}, \frac{1}{6}, \frac{5}{6}\right\}\right. \text {, }\right. \\
& \left.\left[\frac{2}{3}, \frac{2}{3}+\operatorname{RootOf}\left(Z^{2}+1\right)\right]\right],\left[\left\{\frac{1}{2}, \frac{1}{6}, \frac{5}{6}\right\},\left[\frac{1}{3}, 1+\operatorname{RootOf}\left(Z^{2}+1\right)\right]\right], \\
& {\left[\left\{\frac{1}{2}, \frac{1}{6}, \frac{5}{6}\right\},\left[\frac{1}{3}, \operatorname{RootOf}\left(Z^{2}+1\right)\right]\right],\left[\left\{\frac{5}{18}, \frac{11}{18}, \frac{17}{18}\right\},\left[\frac{2}{3}, \operatorname{RootOf}\left({ }_{-} Z^{2}+1\right)\right]\right],} \\
& {\left[\left\{\frac{5}{18}, \frac{11}{18}, \frac{17}{18}\right\},\left[\frac{2}{3}, 1+\operatorname{RootOf}\left(Z^{2}+1\right)\right]\right],\left[\left\{\frac{5}{18}, \frac{11}{18}, \frac{17}{18}\right\},\left[\frac{1}{3}, \frac{1}{3}\right.\right.} \\
& \left.\left.+\operatorname{RootOf}\left(Z^{2}+1\right)\right]\right],\left[\left\{\frac{1}{18}, \frac{7}{18}, \frac{13}{18}\right\},\left[\frac{1}{3}, \frac{2}{3}+\operatorname{RootOf}\left({ }_{-} Z^{2}+1\right)\right]\right],
\end{aligned}
$$




$$
\left.\left.-2 \frac{(x-1)^{3}}{x-9}\right]\right]
$$

Our implemented code Hyp1F2Solutions gives us all the transformation parameters, if they exist, and also the upper and lower parameters of ${ }_{1} F_{2}$ :

$>$ Hyp1F2Solutions (L);

$$
\left.\left\{\left[\left[\left[\frac{1}{2}\right],\left[\frac{1}{3}, \operatorname{RootOf}\left(Z^{2}+1\right)\right],[0],[1]\right]\right], 2 \frac{(x-1)^{3}}{x-9}\right]\right\}
$$

Hence, L has ${ }_{1} F_{2}$ type solutions with hypergeometric parameters $a_{1}=1 / 2, b_{1}=1 / 3$ and $b_{2}=\operatorname{RootOf}\left(x^{2}+1\right)= \pm i$, and transformation parameters: $r=0, r_{0}=1, r_{1}=0$, $r_{2}=0$ and $f=\frac{2(x-1)^{3}}{x-9}$.

\section{${ }_{0} F_{2}$ type solutions}

Let us consider the differential operator $\mathrm{L}_{02}$ associated to the ${ }_{0} F_{2}$ hypergeometric function with lower parameters $b_{1}=1 / 3$ and $b_{2}=1 / 7$. To get $\mathrm{L}_{02}$ with Maple we proceed as follows:

$>$ eq:=sumdiffeq (hyperterm([],[b1,b2],x,i),i, $J(x))$;

$$
\begin{gathered}
e q:=\left(\frac{d^{3}}{d x^{3}} J(x)\right) x^{2}+(b 2+1+b 1)\left(\frac{d^{2}}{d x^{2}} J(x)\right) x-J(x)+b 2 b 1 \frac{d}{d x} J(x)=0 \\
>\quad \text { LA }:=\operatorname{de} 2 \operatorname{diffop}(\text { eq }, \mathrm{J}(\mathrm{x})) ; \\
L A:=x^{2} D x^{3}+(x b 2+x+x b 1) D x^{2}+b 2 b 1 D x-1
\end{gathered}
$$

$>\operatorname{L} 02:=\operatorname{subs}(\{\mathrm{b} 1=1 / 3, \mathrm{~b} 2=1 / 7\}, \mathrm{LA})$;

$$
L 02:=x^{2} D x^{3}+\frac{31}{21} x D x^{2}+\frac{1}{21} D x-1
$$

Let us apply to $\mathrm{L}_{02}$ the change of variables transformation with parameter $f$ given by

$>\quad f:=(2 *(x-1) \wedge 2 *(x-3) *(x-7)-3) /((x-9) \wedge 2 *(x-12) \wedge 3) ;$

$$
f:=2 \frac{(x-1)^{2}(x-3)(x-7)^{3}}{(x-9)^{2}(x-12)^{3}}
$$

That gives us the following operator called L:

$$
\begin{aligned}
&>\quad \mathrm{L}:=\text { ChangeOfVariables }(\mathrm{L} 02, \mathrm{f}) ; \\
& L:= 21(x-12)^{6}(x-9)^{5}(x-7)^{2}(x-3)^{2}(x-1)^{2}\left(5193-3852 x-60 x^{3}+830 x^{2}\right. \\
&\left.+x^{4}\right)^{2} D x^{3}+\left(-173735685+300375864 x+32319144 x^{3}-159987168 x^{2}+31 x^{8}\right. \\
&\left.-635762 x^{4}-3720 x^{7}-649176 x^{5}+86200 x^{6}\right) D x^{2}(x-1)(x-3)(x-7)(x-9)^{4} \\
& \times(x-12)^{5}\left(5193-3852 x-60 x^{3}+x^{4}+830 x^{2}\right)+\left(-5387188885607952 x+x^{16}\right. \\
&-6395117622870960 x^{3}+3388591949109444 x^{4}+7748517717658728 x^{2}-240 x^{15} \\
&+54803306488 x^{10}-3065641808 x^{11}-3904976 x^{13}+63960 x^{14}-746349293552 x^{9} \\
&+130520372 x^{12}-56984531313168 x^{7}+7599004335182 x^{8}-1223256100618800 x^{5} \\
&\left.+311300882943048 x^{6}+1632102637284153\right) D x(x-9)^{3}(x-12)^{4}-42(-3852 x \\
&\left.+5193-60 x^{3}+x^{4}+830 x^{2}\right)^{5}(x-7)^{2}(x-1)
\end{aligned}
$$


Let us assume that we have as input this operator $\mathrm{L}$ and then see how with our codes we can find its ${ }_{0} F_{2}$ type solutions if they exist. That means if we can find the hypergeometric parameters $b_{1}$ and $b_{2}$ of ${ }_{0} F_{2}$ and the transformation parameters such that

$$
\mathrm{L}_{02} \stackrel{f}{\longrightarrow} C \mathrm{M} \longrightarrow E G \mathrm{~L} .
$$

Let ext be the set of all non-rational numbers, parameters and names (except the variable $x$ ) which appear in the expression of the coefficients of $\mathrm{L}$ :

$>\operatorname{ext}:=\operatorname{indets}(\mathrm{L},\{\operatorname{Root} 0 f$, name $\}) \operatorname{minus}\{\mathrm{x}, \mathrm{Dx}\}$;

$$
\text { ext }:=\{\}
$$

Let extp be the set of all algebraic numbers given by the RootOf expressions in ext such that all its elements are algebraic over the minimal field containing $\mathbb{Q} \cup$ (ext $\backslash$ extp). Since sometimes we can use in our computations the RootOf expression associated with its index, we will take extp $=\{\}$.

Let us denote by $\mathrm{E}$ the set of all the singularities of $\mathrm{L}$ (we can find $\mathrm{E}$ using our implemented code Singular).

$>E:=$ Singular $(\mathrm{L}, \mathrm{ext})$ :

Then the set of non-apparent singularities of $\mathrm{L}$ is given by

$>\mathrm{F}:=\operatorname{NotAppSing}(\mathrm{L}, \mathrm{E}, \mathrm{ext})$;

$$
F:=[[\infty, \infty],[x-1,1],[x-3,3],[x-7,7],[x-9,9],[x-12,12]]
$$

Let $\mathrm{S}_{\text {irr }}$ be the set of irregular singularities of $\mathrm{L}$ which is the set of poles of $f$.

$>\operatorname{Sirr}:=\operatorname{irrsing} 0 \mathrm{~F} 2(\mathrm{~L}, \mathrm{t}, \mathrm{F}, \mathrm{ext})$;

$$
\begin{aligned}
& \operatorname{Sirr}:=\left[[[\infty, \infty],[x-9,9],[x-12,12]],\left[\left[-\frac{\sqrt[3]{2}}{\sqrt[3]{t}}-\frac{11}{63}, \frac{1}{\left(1 / 42^{2 / 3}-1 / 4 i \sqrt{3} 2^{2 / 3}\right) \sqrt[3]{t}}\right.\right.\right. \\
& \left.-\frac{11}{63}, \frac{1}{\left(1 / 42^{2 / 3}+1 / 4 i \sqrt{3} 2^{2 / 3}\right) \sqrt[3]{t}}-\frac{11}{63}\right],\left[16 / 3 \frac{\sqrt[3]{12}}{t^{2 / 3}}-\frac{22}{63},-\frac{22}{63}+\frac{16384}{9}\right. \\
& \times \frac{1}{(-8 / 3 \sqrt[3]{12}+8 / 3 i \sqrt{3} \sqrt[3]{12})^{2} t^{2 / 3}}, \frac{16384}{9} \frac{1}{(-8 / 3 \sqrt[3]{12}-8 / 3 i \sqrt{3} \sqrt[3]{12})^{2} t^{2 / 3}} \\
& \left.-\frac{22}{63}\right],\left[-15 \frac{\sqrt[3]{242}}{t}-\frac{11}{21}, \frac{15 / 2 \sqrt[3]{242}-15 / 2 i \sqrt{3} \sqrt[3]{242}}{t}-\frac{11}{21},-\frac{11}{21}\right. \\
& \left.\left.+\frac{15 / 2 \sqrt[3]{242}+15 / 2 i \sqrt{3} \sqrt[3]{242}}{t}\right]\right],\left[\left[\frac{1}{\left(1 / 42^{2 / 3}-1 / 4 i \sqrt{3} 2^{2 / 3}\right) \sqrt[3]{t}}+\frac{\sqrt[3]{2}}{\sqrt[3]{t}},\right.\right. \\
& \frac{1}{\left(1 / 42^{2 / 3}+1 / 4 i \sqrt{3} 2^{2 / 3}\right) \sqrt[3]{t}}+\frac{\sqrt[3]{2}}{\sqrt[3]{t}}, \frac{1}{\left(1 / 42^{2 / 3}+1 / 4 i \sqrt{3} 2^{2 / 3}\right) \sqrt[3]{t}} \\
& \left.-\frac{1}{\left(1 / 42^{2 / 3}-1 / 4 i \sqrt{3} 2^{2 / 3}\right) \sqrt[3]{t}}\right],\left[\frac{16384}{9} \frac{1}{(-8 / 3 \sqrt[3]{12}+8 / 3 i \sqrt{3} \sqrt[3]{12})^{2} t^{2 / 3}}\right. \\
& -16 / 3 \frac{\sqrt[3]{12}}{t^{2 / 3}}, \frac{16384}{9} \frac{1}{(-8 / 3 \sqrt[3]{12}-8 / 3 i \sqrt{3} \sqrt[3]{12})^{2} t^{2 / 3}}-16 / 3 \frac{\sqrt[3]{12}}{t^{2 / 3}}, \frac{16384}{9} \\
& \left.\times \frac{1}{(-8 / 3 \sqrt[3]{12}-8 / 3 i \sqrt{3} \sqrt[3]{12})^{2} t^{2 / 3}}-\frac{16384}{9} \frac{1}{(-8 / 3 \sqrt[3]{12}+8 / 3 i \sqrt{3} \sqrt[3]{12})^{2} t^{2 / 3}}\right]
\end{aligned}
$$




$$
\begin{gathered}
{\left[\frac{\operatorname{RootOf}\left(816750+_{-} Z^{3}, \text { index }=2\right)}{t}-\frac{\operatorname{RootOf}\left(816750+_{-} Z^{3}, \text { index }=1\right)}{t},\right.} \\
\frac{\operatorname{RootOf}\left(816750+_{-} Z^{3}, \text { index }=3\right)}{t}-\frac{\operatorname{RootOf}\left(816750+_{-} Z^{3}, \text { index }=1\right)}{t}, \\
\left.\left.\frac{\operatorname{RootOf}\left(816750+{ }_{-} Z^{3}, \text { index }=3\right)}{t}-\frac{\operatorname{RootOf}\left(816750+_{-} Z^{3}, \text { index }=2\right)}{t}\right]\right],
\end{gathered}
$$

$$
\left.\left[\frac{1}{3}, \frac{2}{3}, 1\right]\right]
$$

Let us take $\mathrm{G}$ as the set of elements in $\mathrm{F}$ at which $\mathrm{L}$ has all its exponent differences in $k$. Let $\mathrm{S}_{\text {reg }}$ be the set of elements in $\mathrm{G}$ at which $\mathrm{L}$ has logarithmic solutions or all its exponent differences are not, at same time, in $\mathbb{N}$. All the elements of $S_{\text {reg }}$ belong to the set of the zeroes of $f$, and they are given by

$>$ Sreg:=regsingtrue0F2(L,t, $\operatorname{Sirr}[-1]$, ext);

$$
\begin{gathered}
\text { Sreg := }\left[[[x-1,1],[x-3,3],[x-7,7]],\left[\left[0, \frac{4}{3}, \frac{12}{7}\right],\left[0, \frac{2}{3}, \frac{6}{7}\right],\left[\frac{18}{7}, 0,2\right]\right],\right. \\
\left.\left[\left[\frac{12}{7}, \frac{4}{3},-\frac{8}{21}\right],\left[\frac{6}{7}, \frac{2}{3},-\frac{4}{21}\right],\left[-\frac{18}{7},-\frac{4}{7}, 2\right]\right]\right]
\end{gathered}
$$

$\mathrm{S}_{\text {reg }}$ can be divided into two subsets: the set of non-removable singularities of $\mathrm{L}$ (at least one exponent difference is not an integer or L has logarithmic solution(s)) and a set of removable singularities of $\mathrm{L}$ (all the exponent differences are integers and $\mathrm{L}$ doesn't have logarithmic solution(s)). The set of non-removable singularities of L can also be divided into two sets: the singularities at which L has logarithmic solutions and the singularities at which L doesn't have logarithmic solutions. Hence, let us denote by $\mathrm{RS}_{\text {reg }}$ a set of three sets: the first is the set of non-removable singularities of $\mathrm{L}$ with non-logarithmic solutions, the second is the set of removable singularities of $\mathrm{L}$ in $\mathrm{S}_{\text {reg}}$, and the third is the set of singularities of $\mathrm{L}$ with logarithmic solutions. With our code called Sregseptrue0F2 we can find this set $\mathrm{RS}_{\text {reg: }}$ :

$>$ RSreg:= Sregseptrue0F2(L, Sreg, ext);

$$
\begin{array}{r}
\text { RSreg: }=\left[[[x-1,1],[x-3,3],[x-7,7]],\left[\left[0, \frac{4}{3}, \frac{12}{7}\right],\left[0, \frac{2}{3}, \frac{6}{7}\right],\left[\frac{18}{7}, 0,2\right]\right],\right. \\
\left.\left.\quad\left[\left[\left[\frac{12}{7}, \frac{4}{3},-\frac{8}{21}\right],[]\right],\left[\left[\frac{6}{7}, \frac{2}{3},-\frac{4}{21}\right],[]\right],\left[\left[-\frac{18}{7},-\frac{4}{7}\right],[2]\right]\right]\right],[],[]\right]
\end{array}
$$

Since $\mathrm{RS}_{\mathrm{reg}}[1] \neq[]$, we know some zeroes of $f$. But we are not sure that they represent all the zeroes of $f$ because the exponent differences of $\mathrm{L}$ at elements of NRemS $S_{\text {reg }}$ are in $\mathbb{Q} \backslash \mathbb{Z}$.

In order to make our codes faster, we have implemented one procedure called IrrRegAppsing0F2 which gives at the same time and, by avoiding duplicate computations, in a shorter time all the outputs of the procedures irrsing0F2, regsingtrue0F2 and Sregseptrue0F2.

$>\quad \mathrm{R} 1:=\operatorname{IrrReg} A$ ppsing0F2 ( $\mathrm{L}, \mathrm{t}, \mathrm{E}, \mathrm{ext})$ :

Let $f=A / B$ with $\mathrm{A}$ and $\mathrm{B}$ as in our theoretical part, and $l=1$. We can get the truncated series for $f$ related to the elements of $\mathrm{S}_{\text {irr }}$, the degree of $\mathrm{A}$ denoted $d_{\mathrm{A}}$ and also the number $n$ of linear equations satisfied by the coefficients of $\mathrm{A}$ using our 
implemented code called Sirr1F2info1. It shows us if we are in the "Easy case" or not. If we are not in this case, we can see in which case we are according to the exponent differences of elements in $\mathrm{RS}_{\mathrm{reg}}[1]$.

$>$ info1:= Sirr0F2info1(L,R1[1],R1[2], $x, t, e x t)$;

infol $:=\left[\left[\left[9, x-9,\left[\frac{2048}{9}(x-9)^{-2}\right], 1,\{\},\{\}\right],\left[\infty, x^{-1},[2 x], 1,\{\},\{\}\right],[12, x-12\right.\right.$,

$$
\left.\left.\left.\left[-30250(x-12)^{-3}\right], 1,\{\},\{\}\right]\right], 6,6,(x-9)^{2}(x-12)^{3},(x-9)(x-12)^{2}\right]
$$

We have $n=6$ and $d_{\mathrm{A}}=6$. Hence $n=d_{\mathrm{A}}$ and therefore we cannot solve those equations and get $\mathrm{A}$ : that is not the "Easy case".

According to the exponent differences of the elements of $\mathrm{RS}_{\mathrm{reg}}[1]$, we are here in the rational case. Our code called $f$ ind0F2Rat gives us the set of candidates for $\left[\left\{\left[b_{1}, b_{2}\right]\right\}, f\right]$ (change of variable parameter and also lower parameters of ${ }_{0} F_{2}$ )

$>$ find0F2Rat (L, R1, info1, $x, t, T, e x t)$;

$\left[\left[\left[\left[\frac{4}{21}, \frac{6}{7}\right],\left[\frac{1}{3}, \frac{4}{21}\right],\left[\frac{2}{3}, \frac{6}{7}\right],\left[\frac{1}{7}, \frac{17}{21}\right],\left[\frac{2}{3}, \frac{17}{21}\right],\left[\frac{1}{3}, \frac{1}{7}\right]\right], 2 \frac{(x-1)^{2}(x-3)(x-7)^{3}}{(x-9)^{2}(x-12)^{3}}\right]\right]$

Our implemented code Hyp0F2Solutions gives us all the transformation parameters, if they exist, and also the upper and lower parameters of ${ }_{0} F_{2}$ :

$>$ Hyp0F2Solutions (L);

$$
\left.\left\{\left[\left\{\left[\frac{1}{3}, \frac{1}{7}\right],[0],[1]\right]\right\}, 2 \frac{(x-1)^{2}(x-3)(x-7)^{3}}{(x-9)^{2}(x-12)^{3}}\right]\right\}
$$

Hence, $\mathrm{L}$ has ${ }_{0} F_{2}$ type solutions with hypergeometric parameters $b_{1}=1 / 3$ and $b_{2}=1 / 7$, and transformation parameters: $r=0, r_{0}=1, r_{1}=0, r_{2}=0$ and $f=\frac{2(x-1)^{2}(x-3)(x-7)^{3}}{(x-9)^{2}(x-12)^{3}}$. 


\section{Chapter 6}

\section{Conclusion and Perspectives}

Let $k$ be an extension field of $\mathbb{Q}$ which is algebraically closed and has characteristic zero, and $k(x)[\partial]$ is the ring of differential operators with coefficients in $k(x)$. Let $\mathrm{E}=$ $\left\{\check{\mathrm{B}}_{\nu}^{2},{ }_{1} F_{1}^{2},{ }_{0} F_{2},{ }_{1} F_{2},{ }_{2} F_{2}\right\}$ where $\mathrm{B}_{\nu}$ is the Bessel function (see [2]), $\check{\mathrm{B}}_{\nu}^{2}(x)=\mathrm{B}_{\nu}^{2}(\sqrt{x})$ and ${ }_{p} F_{q}$ with $p \in\{0,1,2\}, q \in\{1,2\}$, the generalized hypergeometric functions (see [40] and [29]). Given an irreducible third-order linear differential operator L without Liouvillian solutions, we have developed algorithms to solve $\mathrm{L}$ in terms of $S \in \mathrm{E}$. We find a solution $y$ (if that exists) of $\mathrm{L}$ in terms of $S \in \mathrm{E}$, change of variables, algebraic operations and exponential integrals:

$$
y=\exp \left(\int r d x\right)\left(r_{0} S(f(x))+r_{1}(S(f(x)))^{\prime}+r_{2}(S(f(x)))^{\prime \prime}\right)
$$

where $r, r_{0}, r_{1}, r_{2}, f \in k(x)$. So we have shown how to find (if they exist) transformations between our given operator $\mathrm{L}$ and some operators coming from $S \in \mathrm{E}$ such that

$$
\mathrm{L}_{S} \stackrel{f}{\longrightarrow} C \mathrm{M} \stackrel{r}{\longrightarrow} \mathrm{L}_{1}{\stackrel{r_{0}, r_{1}, r_{2}}{\longrightarrow}}_{G} \mathrm{~L}
$$

where $\mathrm{M}, \mathrm{L}_{1} \in k(x)[\partial]$.

We start by studying the local information from generalized exponents of L at its nonremovable singularities. That helps us to get partial information about zeroes and poles of $f$. So we can construct a possible list of candidates for $f$, and upper and lower parameters of $S$ when $S \in\left\{{ }_{1} F_{1}^{2},{ }_{0} F_{2},{ }_{1} F_{2},{ }_{2} F_{2}\right\}$ or the Bessel parameter otherwise $\left(S=\check{\mathrm{B}}_{\nu}^{2}\right)$. Since finding $f$ is equivalent to finding $\mathrm{M}$, we can also get a list of candidates for $\mathrm{M}$ related to $f$. We find $r, r_{0}, r_{1}, r_{2} \in k(x)$ by using some information coming from the generalized exponents of $\mathrm{L}$ and $\mathrm{M}$ at their non-removable singularities.

The next steps of this work would be to apply such a formalism to solve some families of difference and $q$-difference equations. 


\section{Bibliography}

[1] S. Abramov, M. Barkatou, and M. van Hoeij. Apparent singularities of linear differential equations with polynomial coefficients. In ISSAC'95 Proceedings, pages 117-133, 2006. 7

[2] M. Abramowitz and I. A. Stegun. Handbook of Mathematical Functions, volume 55 of Applied Mathematics Series. Tenth Printing, 1972. ix, 2, 7, 173

[3] M. A. Barkatou and E. Pflügel. On the equivalence problem of linear differential systems and its application for factoring completely reducible systems. In ISSAC'98 Proceedings, pages 268-275, 1998. 21

[4] A. Bostan, X. Caruso, and E. Schost. A fast algorithm for computing the characteristic polynomial of the p-curvature. arXiv: 1405.5341v1 [cs.SC], France, 21 May 2014. $3,53,73$

[5] A. Bostan, T. Cluzeau, and B. Salvy. Fast algorithms for polynomial solutions of linear differential equations. In ISSAC'2005 Proceedings, pages 45-52, 2005. 21

[6] M. Bronstein. An improved algorithm for factoring linear ordinary differential operators. In ISSAC'94 Proceedings, pages 336-340, 1994. 7

[7] M. Bronstein and S. Lafaille. Solutions of linear ordinary differential equations in terms of special functions. In ISSAC'02 Proceedings, pages 23-28, 2002. 21

[8] B. C. Carlson. Special Function of Applied Mathematics. Academic Press, London, 1977. 7

[9] L. Chan and E. S. Cheb-Terrab. Non-liouvillian solutions for second order linear odes. ISSAC'04 Proceedings, pages 80-86, 2004. 7

[10] T. Cluzeau and M. van Hoeij. A modular algorithm to compute the exponential solutions of a linear differential operator. J. Symb. Comput., pages 1043-1076, 2004. $3,53,73,74$

[11] R. Debeerst. Solving differential equations in terms of Bessel functions. Master's thesis, Universität Kassel, 2007. 7, 12, 21, 27, 95, 98, 102

[12] R. Debeerst, M. van Hoeij, and W. Koepf. Solving differential equations in terms of Bessel functions. ISSAC'08 Proceedings, pages 39-46, 2008. 7

[13] W. N. Everitt and C. Markett. On a generalization of Bessel functions satisfying higher-order differential equations. J. Comput. Appl. Math. 54, 3, pages 325-349, 1994. 7 
[14] W. N. Everitt, D. J. Smith, and M. van Hoeij. The fourth-order type linear ordinary differential equations. arXiv:math/0603516, 2006. 7

[15] T. Fang and M. van Hoeij. 2-descent for second order linear differential equations. ISSAC'2011 Proceedings, pages 107-114, 2011. 7

[16] M. van Hoeij. Factorization of linear differential operators. PhD thesis, Universitijt Nijmegen, 1996. 1, 2, 14, 15, 19

[17] M. van Hoeij. Rational solutions of the mixed differential equation and its application to factorization of differential operators. ISSAC'1996 Proceedings, pages 219$225,1996.1,19,53,78,86$

[18] M. van Hoeij. Factorization of linear differential operators with rational functions coefficients. J. Symbolic Computation 24, pages 237-561, 1997. 1, 19

[19] M. van Hoeij. Finite singularities and hypergeometric solutions of linear recurrence equations. Journal of Pure and Applied Algebra 139, pages 109-131, 1999. 86

[20] M. van Hoeij. Solving third order linear differential equations in terms of second order equations. ISSAC'2007 Proceedings, pages 355-360, 2007. 21, 86

[21] M. van Hoeij and Q. Yuan. Finding all Bessel type solutions for linear differential equations with rational function coefficients. ISSAC'2010 Proceedings, pages 37-44, 2010. 7

[22] P. Horn. Faktorisierung in Schief-Polynomringen. PhD thesis, Universität Kassel, 2008. 1,19

[23] E. L. Ince. Ordinary Differential Equations. Dover Publications Inc., 1956. 7

[24] N. M. Katz. A conjecture in the arithmetic theory of differential equations. Bulletin de la Soc. Math., France, tome 110, pages 203-239, 1982. 3, 53, 73, 74

[25] W. Koepf. Identities for families of orthogonal polynomials and special functions. Konrad-Zuse-Zentrum for Informationstechnik Berlin, Takustr. 7, 0-141 95 Berlin, 15 June 1995. 137, 138

[26] W. Koepf. Hypergeometric Summation-An Algorithmic Approach to Summation and Special Function Identities. Springer, 2014. 7, 86

[27] J. Kovacic. An algorithm for solving second-order linear homogeneous equations. In J. Comp., vol. 2, pages p3-43, 1986. 1

[28] E. Pflügel. An algorithm for computing exponential solutions of first order linear differential system. ISSAC'1997 Proceedings, pages 164-171, 1997. 21

[29] A. P. Prudnikov, Y. A. Brychkov, and O. I. Marichev. Integrals and Series, vol. 3: More Special Functions. Gordon and Breach Science, 1990. ix, 2, 173

[30] M. van der Put. Differential equations in characteristic $p$. In Compositio Mathematica 97 , pages $227-251,1996.3,53,73,74$ 
[31] M. van der Put. Reduction modulo $p$ of differential equations. Indag. Math N.S 7(3), pages $367-387,1996$. 1, 7

[32] M. van der Put and M. F. Singer. Galois Theory of Linear Differential Equations, volume 328. Comprehensive Studies in Mathematics, Springer, Berlin, 2003. 1, 2, $7,8,13,14,53,86$

[33] Salvy, B. and Zimmermann, P.: GFUN: A Maple package for the manipulation of generating and holonomic functions in one variable. ACM Transactions on Mathematical Software 20, 163-177, 1994. 55

[34] A. V. Shannin and V. Craster. Removing false singular points as a method of solving ordinary differential equations. Eur. J. Appl. Math. 13, pages 617-639, 2002. 7

[35] M. F. Singer. Solving homogeneous linear differential equations in terms of second order linear differential equations. Am. J. of Math., 107, pages 663-696, 1985. 1, 21

[36] M. F. Singer and F. Ulmer. Galois groups for second and third order linear differential equations. J. Symb. Comp., 16, No.1, pages 9-36, 1993. 7

[37] M. F. Singer and F. Ulmer. Liouvillian and algebraic solutions of second and third order linear differential equations. J. Symb. Comp. 16, pages 37-73, 1993. 7

[38] S. Y. Slavyanov and W. Lay. Special Functions, A Unified Theory Based on Singularities. Oxford Mathematical Monographs, 2000. 7

[39] R. P. Stanley. Differentiably finite power series. Europ. J. Combinatorics 1, pages 175-188, 1980. 55

[40] Z. X. Wang and D. R. Guo. Special Functions. World Scientific Publishing, Singapore, 1989. ix, 2, 7, 173

[41] B. L. Willis. An extensible differential equation solver. SIGSAM Bulletin 35, 1, pages 3-7, March 2001. 7

[42] Q. Yuan. Finding all Bessel type solutions for linear differential equations with rational function coefficients. PhD thesis, Florida State University, 2012. 3, 7, 21, $53,73,75,102,110,111,129,130,134,135$ 


\section{Index}

$\mathrm{NS}_{\text {reg }}(\mathrm{L}), \operatorname{RemS}_{\mathrm{reg}}(\mathrm{L}), \mathcal{A}, 120$

$\mathrm{S}_{\mathrm{log}}(\mathrm{L}), \mathrm{S}_{\mathrm{reg}}(\mathrm{L}), \mathrm{S}_{\mathrm{irr}}(\mathrm{L}), \overline{\mathrm{S}_{\text {reg }}(\mathrm{L})}, \overline{\mathrm{S}_{\text {irr }}(\mathrm{L})}, 72$ $\overline{\mathrm{NS}_{\text {reg }}(\mathrm{L})}, \overline{\operatorname{RemS}_{\text {reg }}(\mathrm{L})}, \overline{\mathcal{A}}, 120$

BesSqRootSolutions, 131

BesSqSolutions, 105

EquivExpgauge0F2, 87

EquivExpgauge1F1sq, 87

EquivExpgauge1F2, 87

EquivExpgauge2F2, 87

EquivExpgaugeBesSq1, 87

EquivExpgaugeBesSqRoot1, 87

Hyp0F2Solutions, 165

Hyp1F1sqSolutions, 154

Hyp1F2Solutions, 165

Hyp2F2Solutions, 154

$m^{\text {th }}$ symmetric power of an operator, 86

$p$-curvature of an operator, 74

$p$-curvature test between two operators, 75

algebraic point over a field, 8

analytic function at a point, 9

apparent singularity of an operator, 9

Beke's algorithm, 1

Bessel functions, 10

Bessel operator, 12

Bessel's differential equation, 10

Bessel's parameter, 10

change of variables, 2

complete solver, 2

constant field, 7

degree, 8

derivation, 7

differential field, 7

Digamma fuction, 11

Euclidean ring, 8

Eulerian solutions, 1

exp-product, 2

exp-product equivalent, 75 exponent, 15

exponent difference, 67

exponential solution of an operator, 86

field of formal Laurent series, 7

field of rational functions, 7

formal solutions, 15

fundamental system of power series solutions of an operator, 9

gauge equivalent, 78

gauge transformation, 2

generalized exponent, 15

generalized hypergeometric series, 10

greatest common divisor, 8

greatest common left divisor, 8

greatest common right divisor, 8

hyperexponential functions, 12

hypergeometric differential equation, 10

hypergeometric function, 10

indicial equation, 17

irreducible operator, 1

irregular singularity of an operator, 9

irregular solution of an operator, 17

Kovacic's algorithm, 1

leading coefficient, 8

least common left multiple, 8

least common multiple, 8

least common right multiple, 8

linear $S$-expression, 1

linear differential operator, 8

Liouvillian solutions, 1

local parameter, 9

logarithmic solution, 14

modified Bessel equation, 11

modified Bessel functions, 12

modified Bessel operator, 12

multiplicity order of a pole of a function, 39 
multiplicity order of a zero of a function, 39

non-removable singularity, 2

order, 8

Ordinary differential equations, 1

Pochhammer symbol, 10

polar part of a function, 80

polar part of a function at its pole, 80

pole of a function, 39

projective equivalent, 80

Puiseux series, 15

ramification index, 15

ramified generalized exponent, 15

reducible operator, 1

regular function at a point, 9

regular point of an operator, 9

regular singularity of an operator, 9

regular solution of an operator, 17

removable singularity, 2

right division, 8

right divisor, 8

ring of differential operators, 8

singular point of an operator, 9

solution space, 8

solutions of a differential operator, 8

solve a differential operator in terms of a

function, 54

symmetric product of two operators, 86

transformation parameters, 21

transformations, 2

universal extension, 13

unramified generalized exponent, 15

zero of a function, 39 


\section{Selbstständigkeitserklärung}

Hiermit versichere ich, dass ich die vorliegende Dissertation selbständig und ohne unerlaubte Hilfe angefertigt und andere als die in der Dissertation angegebenen Hilfsmittel nicht benutzt habe. Alle Stellen, die wörtlich oder sinngemäß aus veröffentlichten oder unveröffentlichten Schriften entnommen sind, habe ich als solche kenntlich gemacht. Dritte waren an der inhaltlich-materiellen Erstellung der Dissertation nicht beteiligt; insbesondere habe ich hierfür nicht die Hilfe eines Promotionsberaters in Anspruch genommen. Kein Teil dieser Arbeit ist in einem anderen Promotions- oder Habilitationsverfahren verwendet worden. 